\title{
Epitaxial Growth of half-metallic magnetic oxide thin films by Pulsed Laser Deposition
}

Julia María Orna Esteban 

Epitaxial Growth of

half-metallic magnetic oxide thin films

by Pulsed Laser Deposition 


\section{Colección de Estudios de Física}

Vol. 82

Esta colección recoge las tesis presentadas en el Departamento de Física de la Materia Condensada de la Universidad de Zaragoza desde su constitución en $198 \%$. 
Colección de Estudios de Física $\quad$ Vol. 82

\section{Epitaxial Growth of \\ half-metallic magnetic oxide thin films \\ by Pulsed Laser Deposition}

Julia María Orna Esteban

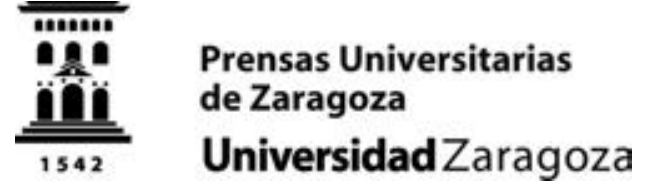


ORNA ESTEBAN, Julia María

Epitaxial Growth of half-metallic magnetic oxide thin films by Pulsed Laser Deposition / Julia María Orna Esteban. - Zaragoza : Prensas Universitarias de Zaragoza, 2010

XVIII, 214 p. ; 24 cm. - (Colección de Estudios de Física ; 82)

Bibliografía: p. 199-214. - ISBN 978-84-15031-68-0

Espintrónica-Tesis doctorales.

621.3.049.77(043.2)

Cualquier forma de reproducción, distribución, comunicación pública o transformación de esta obra solo puede ser realizada con la autorización de sus titulares, salvo excepción prevista por la ley. Diríjase a CEDRO (Centro Español de Derechos Reprográficos, www.cedro.org) si necesita fotocopiar o escanear algún fragmento de esta obra.

(C) Julia María Orna Esteban

(C) De la presente edición, Prensas Universitarias de Zaragoza

1. a edición, 2010

Prensas Universitarias de Zaragoza. Edificio de Ciencias Geológicas, c/ Pedro Cerbuna, 12, 50009 Zaragoza, España. Tel.: 976761 330. Fax: 976761063

puz@unizar.es http://puz.unizar.es

Impreso en España

Imprime: Servicio de Publicaciones. Universidad de Zaragoza

D.L.: Z- 3375/2010 
A mi familia:

Juanjo y Filo, Isabel y Alfredo, y a Javi sin vosotros no habría sido posible 



\section{Contents}

Preface xiii

Acronyms xvii

Introduction 1

Nanotechnologv . . . . . . . . . . . . . . . . . . . . . 2

Epitaxial thin film growth . . . . . . . . . . . . . . . . . 4

Epitaxial growth . . . . . . . . . . . . . . . . 5

Film nucleation and growth $\ldots \ldots \ldots \ldots$

Substrate effects ... . . . . . . . . . . . . . 8

Applications of thin films in electronics. . . . . . . . . . . . . . 9

Spintronics: GMR \& TMR . . . . . . . . . . . . . . . . . . . 12

Half metallic materials . . . . . . . . . . . . . . . . . . . . 17

1 Experimental Methods 19

1.1 Epitaxial thin film growth systems . . . . . . . . . . . . . . . 19

1.1 .1 Pulsed Laser Deposition . . . . . . . . . . . . . . . . 21

1.1 .2 Sputtering . . . . . . . . . . . . . . . . . . 32

1.2 Structural and Morphological Characterization . . . . . . . 38

1.2 .1 X-rav Diffraction . . . . . . . . . . . . . . . . . 38

$1.2 .2 \quad$ X-rav Reflectivity . . . . . . . . . . . . . . . . . . . 41

1.2 .3 Atomic Force Microscopy . . . . . . . . . . . . . . . . . 43

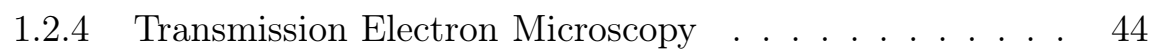

1.3 Magnetic Characterization . . . . . . . . . . . . . . . . . 46

1.3 .1 SQUID magnetometrv . . . . . . . . . . . . . . . . . 46

1.3.2 Vibrating Sample Magnetometry . . . . . . . . . . . . . 47

1.3 .3 Magnetic Force Microscopv . . . . . . . . . . . . . . . . 48

1.3.4 Alternating Gradient Force Magnetometry . . . . . . . . 48

1.3.5 Polarized Neutron Reflectometrv . . . . . . . . . . . . . 49

1.4 Magnetotransport . . . . . . . . . . . . . . . . . . . . 52

1.5 Lithographv . . . . . . . . . . . . . . . . . . . . . . 54

1.5.1 Photolithography standard procedure . . . . . . . . 54 
2 Magnetite epitaxial thin films $\quad 57$

2.1 Magnetite: an overview . . . . . . . . . . . . . 57

2.1.1 Structure and properties of bulk $\mathrm{Fe}_{3} \mathrm{O}_{4} \ldots \ldots . . .258$

2.1.2 Structure and properties of epitaxial $\mathrm{Fe}_{3} \mathrm{O}_{4}$ films . . . . 61

2.2 Growth optimization . . . . . . . . . . . . . . . 67

2.2 .1 Target preparation . . . . . . . . . . . . . 67

2.2 .2 Deposition rate . . . . . . . . . . . . . . . . . . . . 69

2.2 .3 Substrate temperature . . . . . . . . . . . . . 71

2.2 .4 Structural characterization . . . . . . . . . . 73

2.2.5 Morphological and surface characterization . . . . . . 76

2.2.6 Magnetic and electrical transport properties . . . . . . . 79

2.3 A thickness studv . . . . . . . . . . . . . . . . . . . . . . . 81

2.3.1 Morphological and surface characterization . . . . . . 81

2.3 .2 Magnetic behavior . . . . . . . . . . . . . . . . . . . . . . . . . . . . . . . 84

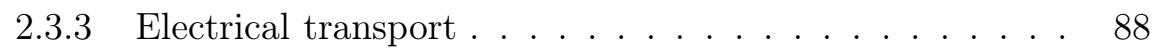

2.3 .4 Magnetotransport . . . . . . . . . . . . . . . . 92

2.4 Enhanced magnetic moment in epitaxial $\mathrm{Fe}_{3} \mathrm{O}_{4}$ ultra-thin films 98

2.4.1 Polarized Neutrons Reflectivity characterization . . . . . 100

2.4.2 X-rav absorption and magnetic circular dichroism . . . . 103

2.4.3 X-ray Photoemission Spectroscopy measurements . . . . 105

2.4 .4 Origin of the enhanced magnetic moment. . . . . . . . 106

2.5 Study of the $\mathrm{Nb}: \mathrm{SrTiO}_{3} / / \mathrm{Fe}_{3} \mathrm{O}_{4}$ Schottky barrier . . . . . . . 110

$\begin{array}{lll}3 & \mathrm{Sr}_{2} \mathrm{CrReO}_{6} \text { epitaxial thin films } & 117\end{array}$

3.1 An introduction to $\mathrm{Sr}_{2} \mathrm{CrReO}_{6}$. . . . . . . . . . . . . . 117

3.1 .1 Double Ordered Perovskites . . . . . . . . . . . . 117

3.1.2 Crvstallographic structure defects: Antisites (AS) . . . 119

3.1.3 Growth of epitaxial $\mathrm{Sr}_{2} \mathrm{CrReO}_{6}$ thin films . . . . . . . . 121

3.2 Growth optimization . . . . . . . . . . . . . . . . . . 122

3.2.1 Target preparation and characterization . . . . . . . 122

3.2.2 A morphological study of the $\mathrm{SrTiO}_{3}(001)$ substrate . . 124

3.2.3 Growth Optimization of $\mathrm{Sr}_{2} \mathrm{CrReO}_{6}$ films. . . . . . . . . 127

3.3 Structural. magnetic and electrical transport characterization . 130

3.4 Transmission Electron Microscopy . . . . . . . . . . . . . . . . 136

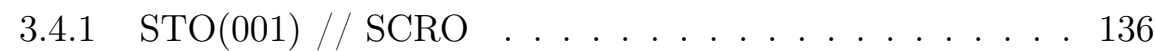

$3.4 .2 \mathrm{STO}(111) / /$ SCRO: cation ordering . . . . . . . . 138

$4 \quad$ FePt epitaxial thin films 143

4.1 Introduction . . . . . . . . . . . . . . . . . . . . . . 143

4.1.1 FePt: a solution to the superparamagnetic limit . . . . . 143

4.1 .2 The FePt allov . . . . . . . . . . . . . . . . . . 145

4.2 FePt film growth . . . . . . . . . . . . . . . . . 147 


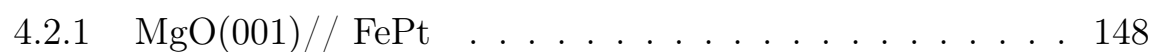

4.2.2 $\mathrm{MgO}(001) / /$ Buffer laver $/ \mathrm{FePt} \ldots$. . . . . . . . . 153

$4.2 .3\left(\mathrm{Lan}_{3} \mathrm{Sr}_{0}\right)\left(\mathrm{Al}_{05} \mathrm{Ta}_{035}\right) \mathrm{O}_{3}(110) / / \mathrm{FePt} \ldots \ldots . . . . .158$

$4.2 .4 \mathrm{STO}(001) / / \mathrm{FePt} \ldots \ldots \ldots \ldots$

$5 \mathrm{Fe}_{3} \mathrm{O}_{4}$ - based heteroepitaxial structures $\quad 167$

$5.1 \mathrm{Fe}_{3} \mathrm{O}_{4} / \mathrm{MgO} / \mathrm{Fe}_{3} \mathrm{O}_{4} / \ldots \ldots \ldots$. . . . . . . . . . . 169

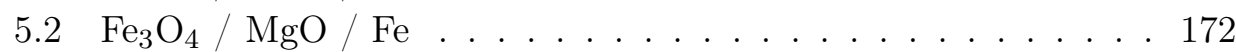

$5.3 \mathrm{Fe}_{3} \mathrm{O}_{4} / \mathrm{MgO} / \mathrm{FePt} \ldots \ldots \ldots \ldots \ldots \ldots . \ldots \ldots 177$

5.3.1 $\mathrm{MgO}(001) / / \mathrm{FePt} / \mathrm{MgO} / \mathrm{Fe}_{3} \mathrm{O}_{4} \ldots \ldots . . . . . . .178$

5.3.2 $\mathrm{MgO}(001) / / \mathrm{Fe}_{3} \mathrm{O}_{4} / \mathrm{MgO} / \mathrm{FePt} \ldots \ldots . . . . .180$

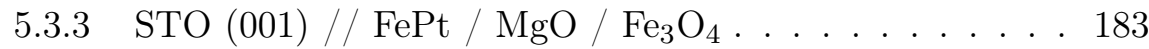

5.4 MTJs: Preliminarv results . . . . . . . . . . . . . . . 186

5.4.1 Magnetic Tunnel Junction Fabrication Process . . . . . 187

5.4 .2 TMR preliminary measurements . . . . . . . . . . . . 193

5.4 .3 MTJs from $\mathrm{Fe}_{3} \mathrm{O}_{4} / \mathrm{MgO} / \mathrm{Fe}$ heterostructures . . . . . . . 195

$\begin{array}{ll}\text { Conclusions } & 197\end{array}$

$\begin{array}{lr}\text { Bibliography } & 199\end{array}$ 



\section{Preface}

Great efforts have been directed towards the research field of Spintronics in the last years after the discovery of the Giant Magnetoresistance effect (GMR) [1, 2]. An essential issue in this field is the obtention of magnetoresistive devices such as Magnetic Tunnel Junctions (MTJs) with half-metallic ferromagnetic materials as active electrodes at room temperature.

Such materials have an energy gap at the Fermi level $\left(\mathrm{E}_{F}\right)$ for one spin direction, therefore, showing a spin polarization of $100 \%$, which optimizes the output signal of the spintronic devices. In order to obtain devices fully operative at room temperature, materials with a high Curie temperature, $T_{C}$, are required. However, as only a few oxides, semiconductors and metallic alloys are candidates to exhibit half-metallicity [3], finding new half-metallic materials is nowadays of great interest. Promising materials are, for example, magnetite and double perovskite oxides. In addition, the problem of the magnetization thermal fluctuation in high-density magnetic storage systems is of recent concern, and could be solved with materials with high magnetocrystalline anisotropy, such as the $\mathrm{L}_{0}$ - ordered FePt.

The growth of thin films and heterostructures based on these materials is an issue of great interest [4]. The epitaxial growth can be achieved by using suitable monocrystalline substrates with similar lattice parameters, by means of different deposition techniques such as laser ablation [5], sputtering [6], molecular beam epitaxy [7], or chemical deposition [8]. The properties of the materials grown by means of these techniques can be tuned depending on different parameters such as the film-substrate lattice mismatch or the epitaxial growth coherence along the film thickness, which offers interesting possibilities. 
The objective of this Thesis has been the growth and characterization of thin films and heterostructures of half-metallic magnetic materials, in which the nanometric-scale phenomena are relevant, with potential applications in magnetoelectronics. Most of the systems studied have been produced by means of a Pulsed Laser Deposition (PLD) and sputtering system. PLD is a suitable deposition technique for the growth of high-quality oxide thin films, and as a PLD-sputtering system by Neocera Inc. company is available at the Instituto de Nanociencia de Aragón (INA) in Zaragoza (Spain), and fully operative since June 2005, this system will represent the fundamental instrument of the Thesis.

This book is composed of five chapters, being the first one dedicated to the description of the experimental techniques used in this research, giving an special importance to the PLD technique.

The second chapter is devoted to the study of magnetite epitaxial thin films. Firstly, the influence of the deposition conditions and thickness on the structural, magnetic and electrical transport properties of magnetite films grown on $\mathrm{MgO}$ substrates are studied. The magnetotransport measurements of the $\mathrm{Fe}_{3} \mathrm{O}_{4}$ thin films were widely and thoroughly studied, allowing the publication of four research papers [I-IV]. Finally, the origin of the enhanced magnetic moment found in magnetite ultra-thin films $(\mathrm{t}<20 \mathrm{~nm})$ [9, 10] was studied and identified, and as a consequence, a research article has been very recently published [V].

The growth and complete characterization of double pervoskite $\mathrm{Sr}_{2} \mathrm{CrReO}_{6}$ (SCRO) thin films deposited on commercial $\mathrm{SrTiO}_{3}$ substrates is detailed in chapter three of this thesis. The obtained results were published in a research article [VI], and a second paper is in process to be submitted [VII].

The fourth chapter deals with the growth and characterization of $\mathrm{L}_{0^{-}}$ ordered FePt epitaxial thin films, grown at the Instituto dei Materiali per l'Ėlettronica ed il Magnetismo (IMEM), Consiglio Nazionale delle Ricerche (CNR) at Parma, during a short stay in the research center.

Finally, the fifth chapter is devoted to the growth and thorough characterization of $\mathrm{MgO}(100) / / \mathrm{Fe}_{3} \mathrm{O}_{4} / \mathrm{MgO} / \mathrm{FM}$ heterostructures, where FM can be $\mathrm{Fe}_{3} \mathrm{O}_{4}$, $\mathrm{Fe}$ or $\mathrm{FePt}$, in order to obtain adequate systems for devices with applications in magnetoelectronics. Finally, preliminary TMR results of MTJs obtained, by means of optical lithography, at the research center INESC-MN in Lisbon (Portugal) are presented. 
La realización de un trabajo de investigación es una carrera de fondo en la que todos los días hay que trabajar con esfuerzo e ilusión, y no puede llevarse a cabo sin el apoyo de las personas que nos rodean. Es por ello que las siguientes líneas están dedicadas a aquellos que me han apoyado y ayudado a madurar y a aprender, como persona y como científico.

En primer lugar quiero agradecer a mis directores de tesis, Luis y Pedro, su paciencia, esfuerzo y dedicación, vuestro trabajo ha sido fundamental. Además de ser unas bellísimas personas, sois unos investigadores excelentes y extraordinarios profesores, que no dudan en remangarse la camisa y bajar a trabajar al laboratorio. Bajo vuestra dirección, no sólo he podido madurar como investigadora, sino que me he sentido realizada al poder trabajar en aquello que me gusta rodeada de un ambiente estupendo.

También estoy especialmente agradecida a los doctores Ricardo Ibarra, Clara Marquina, Rodrigo Fdez-Pacheco y David Serrate. Desde aquel verano de tercero de carrera y durante los siguientes dos años, me hicisteis sentir como en casa, y estoy totalmente segura de que sin vosotros no habría comenzado la carrera investigadora. Gracias. Tampoco debo olvidarme de todos los demás investigadores, tanto del INA como del ICMA y del Departamento, con los que he tenido el placer de trabajar: José Mari, Javier, Anabel, Jasiek, Gerardo, Silvia, Miguel, Luis Miguel, Fernando... siempre que he necesitado ayuda, me la habéis prestado con una sonrisa, gracias también a vosotros, y a César, su trabajo como microscopista ha sido impecable y fundamental para la investigación.

Mil gracias debo dar también, a José Ángel, con el que tanto he aprendido, al "Equipo PLD": Alberto, Lorena, Soraya, Gala, y Laura, y a Noelia. Vais a llegar lejos. Y por supuesto, muchísimas gracias al frente de becarios, de los cuales alguno es ya doctor: Amalio,esta tesis se debe también a tu inestimable trabajo , gracias a Jose Luis, Ana Valera, Teo, Rocío, Luis, gracias a "mis Lauras"... Juana, Rosa, Isabel, Carlos, Rubén y Marisol, vosotros sois una piedra angular en el laboratorio sin la que no habría sido posible este trabajo. Muy agradecida estoy también a toda la gente de secretaría, tanto del INA como del departamento.

Y no debo olvidarme del frente físico, compañeros de tantas fatigas durante la carrera, y después durante el doctorado: Ortiz (Lord Ashcroft), Manu, Jose, Paco, Javi de Lucas... gracias por los cafés y los terapéuticos gabinetes de crisis.

Franca e Francesca, primo di tutto, scusate il mio poverino nivele d'italiano. Grazie per la vostra dedicazione quando sono stata nel laboratorio à Parma, e per i primi lezione d'italiano. Ho cominciato a impararlo con voi, dopo, non ho tenuto tanto tempo di studiarlo, però anche oggi non mi dimentico dei giorni della settimana, neanche dei messi dil anno! Grazie! 
Dejando a un lado la investigación, debo dar las gracias a mis amigas de siempre, que tanto me han apoyado siempre, y más especialmente este último año: Cristina, Maria Pilar, Elena, Ana, Isabel y Julia P. Gracias también a la cuadrilla navarra, que tan bien me ha acogido, y de los cuales no puedo olvidarme en estos folios.

Pero sin duda, si hay algunas personas que se merecen una especial mención, son mi familia. Mamá, papá, gracias por vuestro cariño y vuestro apoyo incondicional, siempre he podido contar con vosotros, y por ello este trabajo es también vuestro. Isabel, muchas gracias por ser más que una hermana, convirtiéndote en confidente y mejor amiga, gracias por tus sabios consejos y por tu cariño, si alguien sabe entenderme, esa eres tú. Y ahora... la familia no sólo queda aquí, también forma parte Alfredo, y Fernando, Jesús y Elena, a la que tanto se echa de menos. Muchas gracias a vosotros también, con vuestro apoyo e interés me habeis animado día a día.

Y por último, Javier. Gracias por todo, siempre consigues hacerme sonreir en mis momentos bajos, y nunca dudas en animarme para seguir adelante en la investigación. Juntos hemos pasado muchas cosas, unas buenas y otras no tanto, estudiado la carrera, llevado a fin el doctorado... y lo mejor está por venir. 


\section{Acronyms}

\begin{tabular}{|c|c|}
\hline AFM & Atomic Force Microscopy \\
\hline $\mathrm{ADF}$ & Annular Dark field \\
\hline AGFM & Alternating Gradient Force Magnetometry \\
\hline AGS & Average Grain Size \\
\hline AHE & Anomalous Hall Effect \\
\hline AMR & Anisotropic Magnetoresistance \\
\hline $\mathrm{APB}$ & Antiphase Boundary \\
\hline CCR & Closed Cicle Refrigerator \\
\hline CNR & Consiglio Nazionale delle Ricerche \\
\hline CVD & Chemical Vapor Deposition \\
\hline CSIC & Consejo Superior de Investigaciones Científicas \\
\hline $\mathrm{DC}$ & Direct Current (Sputtering) \\
\hline DOS & Density of States \\
\hline EELS & Electron Energy Loss Spectroscopy \\
\hline FWHM & Full Width Half Maximum \\
\hline GMR & Giant Magnetoresistance \\
\hline HDD & Hard Disk Drive \\
\hline HR & High Resolution \\
\hline HRXRD & High-Resolution x-ray diffractometer \\
\hline i-CCD & Intensified Charge Coupled Device \\
\hline ICMA & Instituto de Ciencia de Materiales de Aragón \\
\hline ICP & Inductively Coupled Plasma Mass Spectrometry \\
\hline IMEM & Instituto dei Materiali per l'Èlettronica ed il Magnetismo \\
\hline INA & Instituto de Nanociencia de Aragón \\
\hline MBE & Molecular Beam Epitaxy \\
\hline ) $\mathrm{MFM}$ & Magnetic Force Microscopy \\
\hline MIG & Metal in Gap \\
\hline MOKE & Magneto Optic Kerr Effect \\
\hline MR & Magnetoresistance \\
\hline MTJ & Magnetic Tunnel Junction \\
\hline $\mathrm{OHE}$ & Ordinary Hall Effect \\
\hline
\end{tabular}


PHE Planar Hall Effect

PLD Pulsed Laser Deposition

PNR Polarized Neutron Reflectometry

PPMS Physical Properties Measurement System

RF Radio Frequency (Sputtering)

RMS Root-Mean-Square (Roughness)

RSM Reciprocal Space Map

RSO Reciprocating Sample Option

SNR Signal to Noise Ratio

SQUID Superconducting Quantum Interference Device

SVG Silicon Valley Group

TEM Transmission Electron Microscopy

TMR Tunnel Magnetoresistance

VSM Vibrating Sample Magnetometer

XAS X-ray Absorption Spectroscopy

XMCD X-ray Magnetic Circular Dichroism

XPS X-ray Photoemission Spectroscopy

XRD X-ray Diffraction

XRR X-ray Reflectivity 


\section{Introduction}

The motivation of this thesis is found within the newborn research field of nanotechnology, which presents a large number of applications in a wide variety of scientific areas.

Different nanostructures such as nanodots, nanoparticles and thin films have been developed and studied in the last years, and among them, thin films are of the greatest importance in the fields of electronics and magnetoelectronics.

In the last decades, a fruitful research in thin films and thin-film-based devices has taken place, and efforts have been directed towards the new field of spintronics, in which both the charge and spin of the electron are controlled. As a result, new phenomena such as GMR in spin valves [1, 2], and tunnel magnetoresistance (TMR) [11, 12] in magnetic tunnel junctions (MTJs) have been discovered and applied in hard disks and magnetic random access memories.

Focusing on TMR, the optimization of MTJs requires, in addition to high crystalline quality tunnel barriers and electrodes, the use of half-metallic ferromagnetic electrodes at room temperature. In order to obtain devices operating at room temperature, materials with a high Curie temperature $\mathrm{T}_{C}$ are required. However, just a few oxides, semiconductors and metallic alloys are candidates to exhibit half metallicity [3], and therefore, finding new half-metallic materials has became a relevant issue nowadays.

Therefore, the growth of thin films and heterostructures based on these materials has a great importance [4]. An epitaxial growth can be achieved by using adequate monocrystalline substrates with a lattice parameter similar to the grown material, by means of different deposition techniques such as laser ablation [5], sputtering [6], molecular beam epitaxy [7] and chemical deposition [8] among others. The properties of the materials grown by these techniques can be tuned depending on parameters such as the substrate-film lattice mismatch or coherence of the epitaxial growth along the film thickness, which leads to interesting possibilities. 


\section{Nanotechnology}

Nanotechnology, is a new area of science which studies the developing and production of extremely small tools and machines by controlling matter at an atomic and molecular scale. The term "nano" derives from the Greek word for dwarf and is used as a prefix for any unit such as a second or a meter, meaning a billionth of that unit. Hence, a nanometer $(\mathrm{nm})$ is a billionth of a meter $\left(10^{-9}\right.$ $\mathrm{m}$ ). The systems involved in nanotechnology have a size scale between 1 and $100 \mathrm{~nm}$ in at least one dimension. Figure 1 provides a perspective of the "Nanoscale".

The possibility of direct and precise manipulation of individual atoms and molecules was brought up by Richard Feynman, in his lecture at the American Physical Society meeting at Caltech on December 29, 1959, entitled: "There's Plenty of Room at the Bottom". Although he never explicitly mentioned the word "nanotechnology", he suggested the possibility to create "nano-scale" machines with a wide variety of applications, being particularly interested in the possibilities of denser computer circuitry, and microscopes which could image such small systems [13].

The term "nanotechnology" was first defined in 1974 by Norio Taniguchi (University of Tokyo) in a paper as follows: "Nano-technology" mainly consists of the processing of separation, consolidation, and deformation of materials by one atom or one molecule, to refer to a multitude of rapidly emerging technologies, based upon the scaling down of existing technologies to the next level of precision and miniaturization [14]. Taniguchi approached nanotechnology from what is nowadays known as the "top-down" perspective.

In 1986, K. Eric Drexler, at the Foresight Nanotech Institute for Molecular Manufacturing, popularized the term "nanotechnology" thanks to his fictional writings about the construction of machines built at the nanoscale, such as miniature submarines capable of being transported through the human body. Drexler developed, thus, the concept of the assembler, a tiny machine programmed to build objects atom by atom, introducing a new "bottom-up" approach to nanotechnology, instead of the top-down approach discussed earlier by Feynman and Taniguchi. The bottom-up approach involved molecular manipulation and molecular engineering to build molecular machines and molecular devices with atomic precision.

Therefore, there are two different approaches to nanotechnology in terms of techniques for manufacturing nanoscale materials: "bottom-up" or "molecular nanotechnology", which applies to building organic and inorganic structures atom-by-atom or molecule-by-molecule, and "top-down", which refers to making nanoscale structures by machining and etching techniques. 


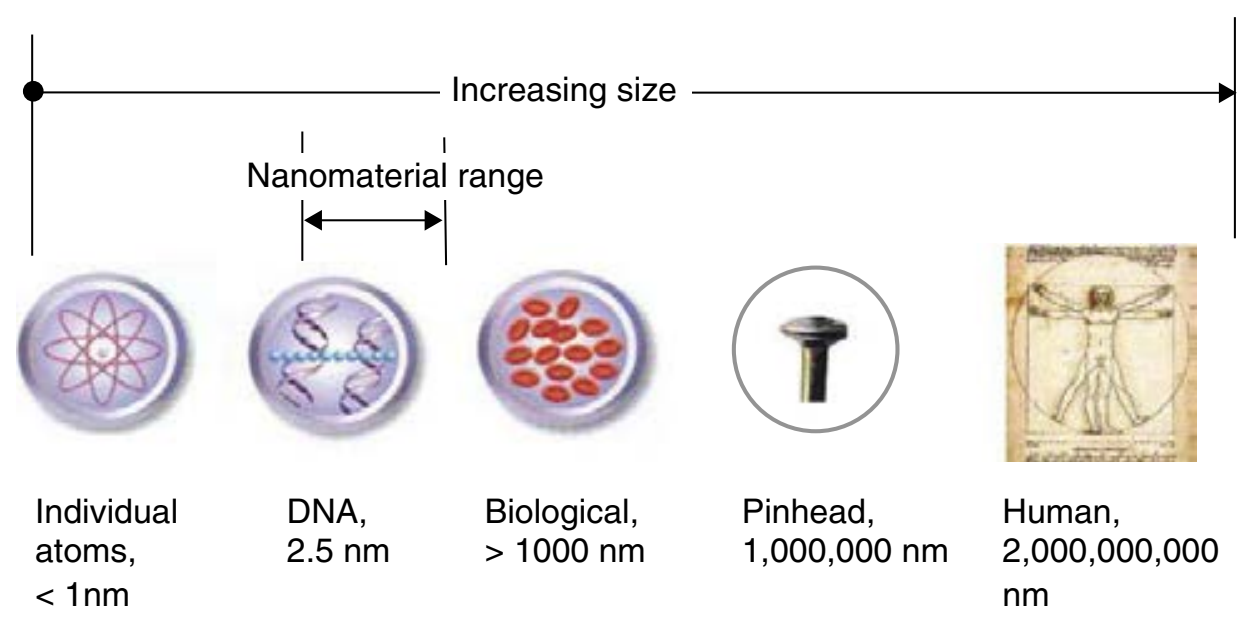

Figure 1: Sequence of images showing the various levels of scale [15].

This new research field has became an interdisciplinary science due to its large number of applications in a wide variety of areas such as medicine, biotechnology, advanced materials, communication technology and environment research among others, which has favored the fast development of manufacturing techniques and manipulation tools. Nanoscale provides a wide range of possibilities:

- By patterning matter at the nanometer length scale, it is possible to vary their mechanical, thermal, electrical, magnetic, optical, and aesthetic properties without changing its chemical composition.

- It is possible to place artificial components and assemblies inside cells, and to make new materials using the self-assembly methods of nature.

- The high surface areas of the nanoscale components make them suitable for its use in composite materials, reacting systems, drug delivery, and energy storage.

- Surface tension increases its importance in small-sized materials, leading to the obtention of harder materials.

- The sizes at the nanoscale become comparable to the interaction wavelength scales of various wave phenomena, making these materials suitable for various opto-electronic applications.

The nanostructures studied in this thesis have been obtained by depositing epitaxial films and heterostructures and performing a subsequent lithography process, thus, approaching the nanoscale with the top-down method and focusing in the thin film branch of nanotechnology and materials science. 


\section{Epitaxial thin film growth}

In thin film deposition, a thin layer with less than a few micrometers in thickness of one substance is placed on another called substrate, either in order to modify the substrate material properties or due to the thin film importance by itself in research fields such as microelectronics or spintronics. The evolution of deposition methods in vacuum environment has allowed the obtention of very high pure films or even multilayers of different materials, which can exhibit properties different from those possessed by any of the bulk components.

This fact has directed great interest to the thin film epitaxial growth, were the films deposited maintain a single-crystalline structure that "fits" on the crystal structure of the substrate thanks to the high purity of the deposition environment, and a correct choice of the substrate combined with its proper cleaning and preparation and suitable deposition conditions.

Thin film deposition is most often developed in closed chambers, and frequently under vacuum conditions. In this process, the addition of atoms or molecules to the substrate can be performed by means of physical vapor deposition, in which atoms or molecules of the source material are vaporized by physical processes such as thermal evaporation and sputtering, and chemical vapor deposition (CVD) techniques, in which a chemical reaction involving one or more vapor species incident on the substrate produce a film of the desired composition. The atoms which arrive at the substrate at a determined rate, depending on the deposition parameters, can diffuse over the substrate or the growing film, find other mobile film atoms to form mobile or stationary clusters, attach to a pre-existing film cluster, be re-evaporated from the substrate or from a cluster, or be dissociated from a cluster and remain on the substrate surface (figure 21). The clusters on the substrate surface will continue their growth until the substrate surface is completely covered, forming the film.

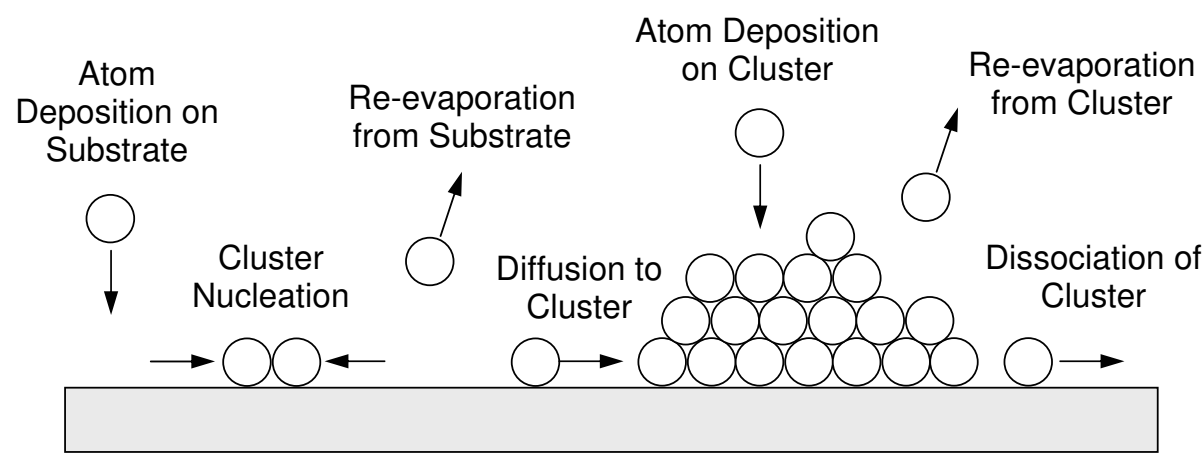

Figure 2: Diagram of possible atomic processes on a substrate during film deposition. 
Therefore, thin film formation is a complex process which can be considered to involve four main stages:

Adsorption: The atoms or molecules directed towards the substrate are attached to its surface by means of physical or chemical adsorption, with a binding energy of $0.25 \mathrm{eV}$, or 1 to $10 \mathrm{eV}$, respectively.

Nucleation: The adsorbed atoms attach to each other forming clusters.

Coalescence: The number of clusters on substrate surface increase until they attach to each other forming bigger clusters until the film is completed.

Crystallization: The film continue its growth while its structure can change depending on the deposition parameters, such as temperature and background gas pressure.

\section{Epitaxial growth}

The word "epitaxy" comes from the Greek terms epi (over) and taxis (located). It was introduced in material science in first place by Royer in 1928, describing the extended single-crystal growth of a film on a crystalline substrate [16].

Epitaxial growth is one of the most important methods to fabricate various electronic and optical devices which require complex structures, as they are usually made of thin layers with various compositions. Quality, performance and lifetime of these devices are determined by the purity, structural perfection, homogeneity, surface flatness and interface abruptness of the epitaxial layers, which depend on factors such as the substrate-film interfacial energy, substrate choice, layer growth method and growth parameters.

Two types of epitaxial growth can be distinguished:

Homoepitaxy: The film is grown upon a crystalline substrate of the same material.

It has the advantage that the substrate and the film have the same lattice constant. Therefore, if the substrate surface is clean of contamination, the epitaxial growth is easy to achieve. This epitaxial growth is specially useful in the growth of semiconductors, in which the films have different properties from the substrate due to a different doping concentration.

Heteroepitaxy: The film and the substrate are different materials.

This type of epitaxial growth involves mismatch between the film and the substrate, although a clean and ordered substrate provides a strongly orientating template for deposited atoms. 
If $a(s)$ is the substrate lattice parameter, and $a_{0}(f)$ the lattice parameter of the film material without stress, i.e. the bulk value, then the mismatch (f) can be defined as:

$$
f=\frac{a(s)-a_{0}(f)}{a_{0}(f)} \times 100
$$

As $\mathrm{f}$ is measured in $\%$, if $a_{0}(f)>a(s) \Rightarrow f<0$ compressive, whereas if $a_{0}(f)<a(s) \Rightarrow f>0$ tensile. If the lattice mismatch is small $(|f|<0.2 \%)$ epitaxial growth occurs easily. However, for larger lattice mismatch the growth is often "pseudomorphic" at the first stages (fig. 3-c), where the film grows with the lattice constant of the substrate resulting in strain in the plane interface. As the film increases in thickness, so does the total strain energy in the strained film, dislocations can be generated as a method of strain relaxation, and the epitaxial growth can become rough on an atomic scale. Finally, for lattice mismatch over $5 \%$ the growth is likely to be relaxed (fig. 3-b) or even polycrystalline [17].

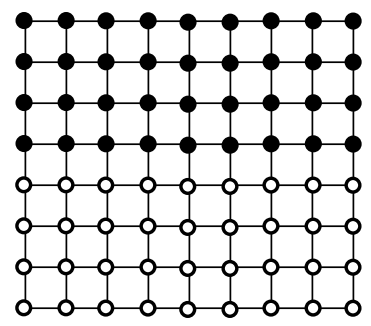

(a)

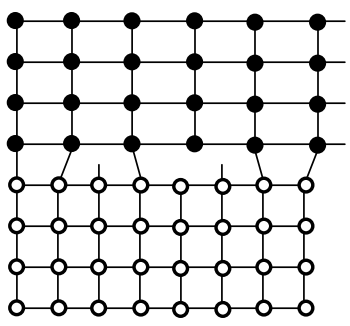

(b)

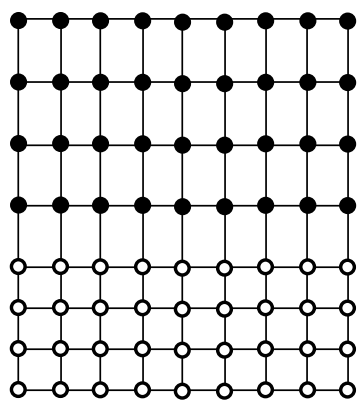

(c)

Figure 3: Schematic cross section of epitaxial growth with different mismatch. (a) Growth with lattice matching. (b) Strain-relaxed growth. (c) Pseudomorphic growth with uniaxial expansion normal to growth interface. Adapted from ref. [18].

\section{Film growth modes}

In 1958 however, Ernst Bauer classified the primary thin film growth process in three modes [19]: three dimensional island growth (Volmer-Weber), two dimensional full monolayer growth (Frank-van der Merwe), and two dimensional growth of full monolayers followed by nucleation and growth of three dimensional islands (Stranski-Krastanov) (figure 4). The selection of one of these growth modes depends on the thermodynamics relating the film and substrate surface energies and the film-substrate interface energy [20]. 


\section{- Volmer-Weber mode: 3D island growth}

Once an island is formed an additional island will nucleate on top of it, and, therefore, the surface roughens each time material is added.

\section{- Frank-van der Merwe mode: 2D full-monolayer growth}

Also called layer-by-layer growth, in this growth mode it is energetically more favorable for the film to form as full monolayers than for threedimensional clusters on the substrate. Therefore, islands nucleate on the surface until a critical island density is reached, and as more material is added, the islands continue to grow until they begin to run into each other (coalescence). Once coalescence is reached, the surface has a large density of pits, and when additional material is added to the surface the atoms diffuse into these pits to complete the layer. This process is repeated for each subsequent layer.

- Stranski-Krastanov mode: 2D full-monolayer growth followed by $3 \mathrm{D}$ island nucleation and growth

This growth mechanism was first noted by Ivan Stranski and Lyubomir Krastanov in 1939 [21]. It is also known as layer-plus-island growth, and follows a two step process. Initially, the film atoms form complete monolayers on the substrate, following a layer by layer growth up to a critical layer thickness which is typically of one to five monolayers. Beyond this critical thickness, three-dimensional clusters nucleate on these layers. This change in the growth mechanism can be explained as a consequence to the stress due to mismatched lattice spacings.
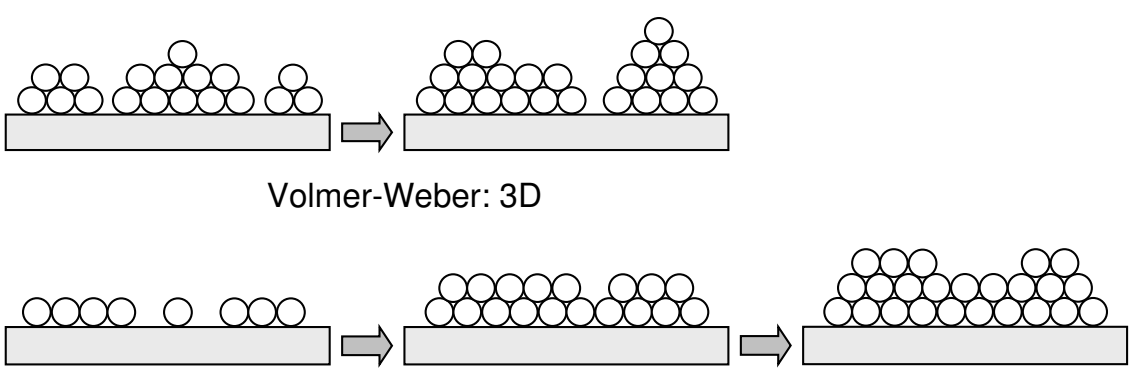

Frank-van der Merwe: 2D

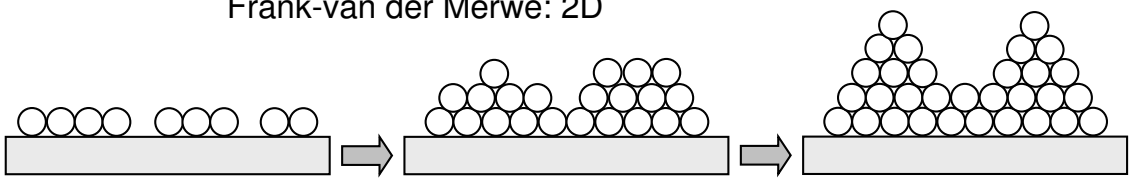

Stranski-Krastinov

Figure 4: Schematic diagram of the basic thin film growth modes. Figure adapted from ref [18]. 


\section{Substrate effects}

Substrates provide the template for the film, and therefore, they should be chosen consequently with the desired thin film material. In addition to substrate structure, substrate surface has an important influence on the film nucleation and growth process, as low energy sites at which nucleation preferentially occurs are provided by surface defects such as atomic steps, point defects and dislocation intersections. Their distribution will control the nucleation rate and nuclei density, which makes a control of the substrate surface quality necessary. However, substrate treatments are sometimes performed in order to induce surface terraces or holes which would act as nucleation sites, leading to a certain growth mode. One example can be found in the different treatments performed in $\mathrm{SrTiO}_{3}$ substrates in order to modify its surface structure [22 28].

If the substrate surface is contaminated with a fraction of a monolayer of impurity atoms, the surface mobility of the arriving film atoms may be reduced affecting the film crystallinity to an extent that the final film can be polycrystalline [18]. Regarding impurities removal from the substrate surface, acetone will eliminate organic residues, and isopropanol will leave the substrate surface clean of acetone residues and other inorganic molecules. As some substrates such as $\mathrm{MgO}$ are sensitive to humidity, it is recommendable the use of dry acetone and isopropanol, with the lower water content possible. However, sometimes the substrate surface is deeply damaged or contaminated and a physical or chemical etching process is necessary, followed by a thermal treatment in order to recover the crystalline quality of the substrate surface.

During the deposition process, the substrate surface temperature plays an important role in the film morphology, as the mobility of the deposited atoms on the surface depends directly on the temperature and can determine the growth mode [18]. The substrate temperature can produce also an improvement of the crystalline structure by defects-diffusion. 


\section{Applications of thin films in electronics}

Thin films have multiple applications in industry, such as thermal barrier coatings and wear protections, which enhance service life of tools and protect materials against thermal and atmospheric influences, catalysis, thin-film batteries, optical coatings and electronic semiconductor devices. We will focus on the thin films application in electronics and magnetoelectronics, fields with a large impact in nowadays society.

One of the key applications of thin films made of magnetic materials is as components of Hard Disk Drive, also named Hard Disk, Hard Drive or HDD. An image of a standard HDD and its main components is shown in figure 5 .

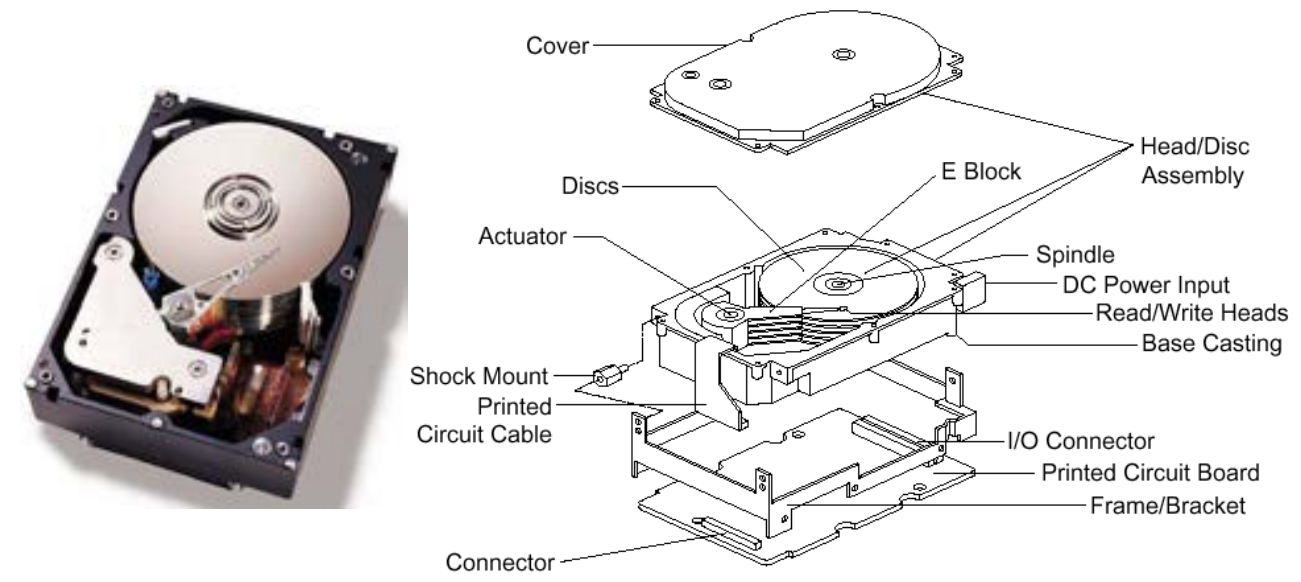

Figure 5: Standard HDD image (left) and sketch with its main components (right) [29].

\section{Thin-film-based devices for HDD components}

\section{HDD disks}

Magnetic thin films are used in HDD as data storage support. The spinning plates that compose standard HDD are coated with a ferromagnetic thin film which is divided into many small sub-micrometer-sized magnetic regions, each of which is used to encode a single binary unit of information. Initially, the magnetic regions were oriented horizontally, but around 2005 the orientation was changed to perpendicular. The advances in materials science and engineering, including nanotechnology, have allowed the exponential increase of data storage capacity, which was initially predicted by Moore in 1965 [30]. Figure 7) shows the original Moore's empirical law: 


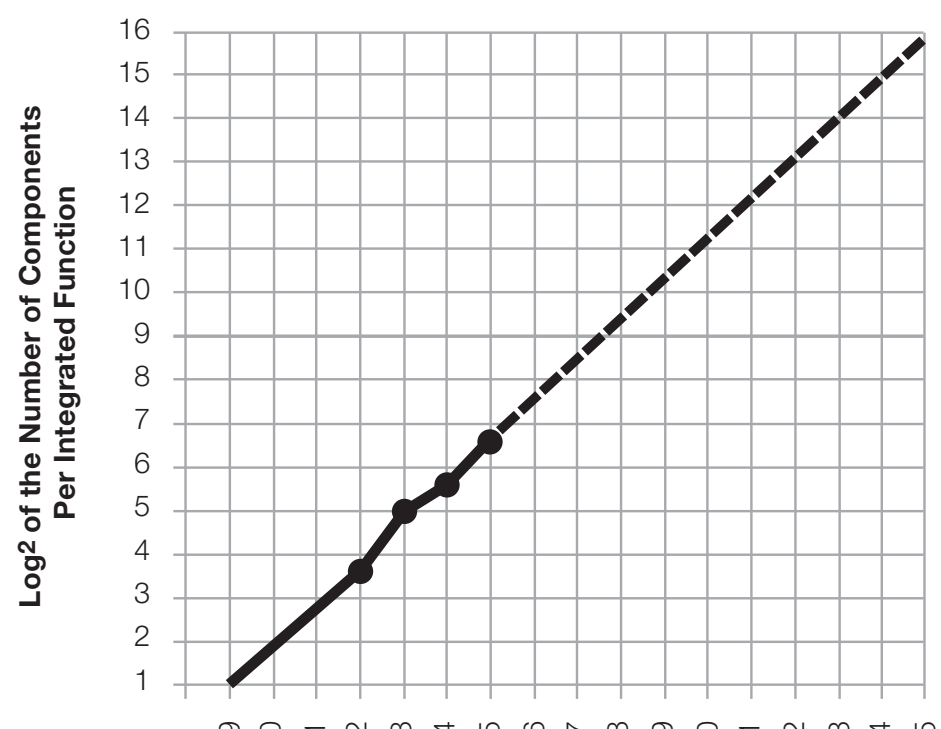

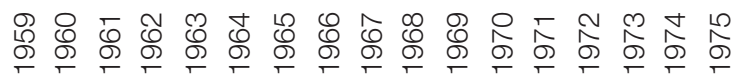

Year

Figure 6: Areal density of data capacity following Moore's Law [30].

\section{Write - Read heads}

These devices are another HDD component that nowadays uses magnetic thin films.

Early HDDs used an electromagnet both to magnetize the region and to read its magnetic field by using electromagnetic induction. The next generation heads were the Metal in Gap (MIG) ones, which incorporated a small piece of metal that concentrated the field, allowing smaller features to be read and written.

MIG heads were later replaced with thin film heads manufactured by photolithographic processes which used the same physics but allowed higher data storage density, obtaining up to $4 \mathrm{~GB}$ storage capacities in 3.5 inch drives in 1995.

The next improvement consisted in the separation of the write-read heads into a thin film head for writing and a head for reading. The new separated read heads, known as AMR heads (Anisotropic Magnetoresistance heads), were developed by Honeywell in the 1980s [31, 32]. The AMR heads used the magnetoresistive (MR) effect, which consists on the change of a material's resistance in the presence of a magnetic field. Such read heads led to a period of fast data storage density increase of about $100 \%$ per year. 
A new path leading to the integration of magnetic devices into computer technology began to emerge with the discovery of GMR in magnetic multilayers in 1988, simultaneously by Baibich et al. (Albert Fert's group in Orsay) [2] and Binash et al. (Peter Grünberg's group in Jülich) [1]. This issue opened a relatively new branch of physics concerned with the storage and transfer of information by means of electron spins in addition to electron charge as in conventional electronics, named as Spintronics, and which is nowadays in considerable expansion.

The first significant GMR device was the spin valve. In the early 1990s IBM started a project to develop such GMR devices into read-write heads, which revolutioned the magnetic HDD industry by enabling the density of the drives to be increased at a much higher rate than was possible with previous technology [33, 34]. Thus, in 2000 GMR heads started to replace AMR read heads, and nowadays, nearly all HDD in the industry incorporate a GMR read-head.

Current research is devoted to a new type of MR magnetic sensor device, known as magnetic tunnel junction (MTJ), which was introduced in first place in 2005 by Seagate, allowing 400 GB drives with 3 disk platters, and uses the TMR.

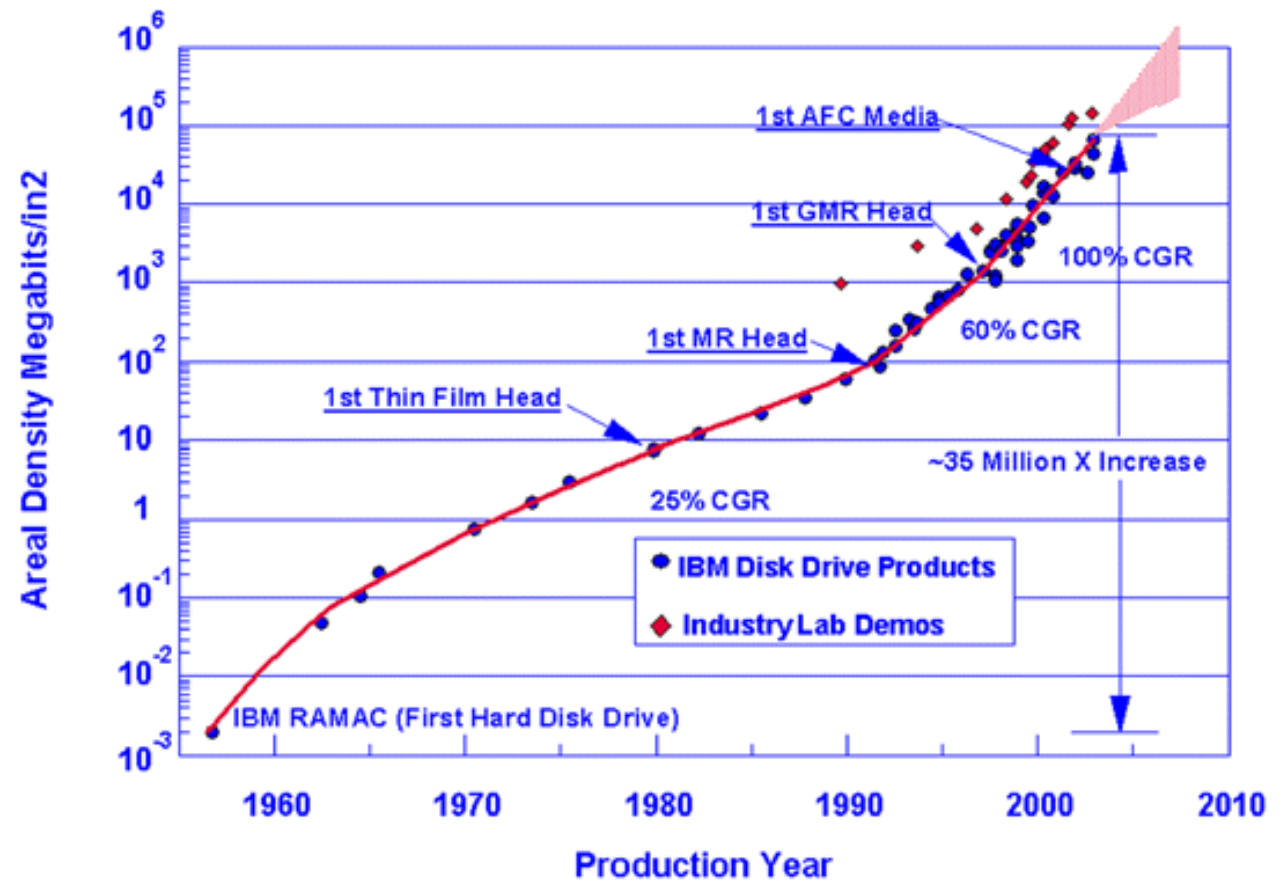

Figure 7: Hard disk areal density trend [35]. 


\section{Spintronics: GMR \& TMR}

Spintronics has became subject of growing interest due to the new functionalities, provided when using both charge and spin degrees of freedom of the electron simultaneously, like non-volatility of the stored information, high data processing speed, low electric power consumption, and high integration densities [34, 36 39]. However, incorporating spin degrees of freedom into an efficient device involves the obtention of high spin-polarized currents, as well as its transport, control and manipulation.

The large research field of Spintronics was opened by the discovery of the GMR effect of magnetic multilayers, which triggered the research on spin transport in magnetic nanostructures. Other interesting effects rapidly appeared, being one of the most important the TMR of MTJ.

\section{Giant Magnetoresistance}

As it was pointed out previously, GMR was discovered by Baibich et al. [2] and simultaneously by Binash et al. [1] in 1988. In both cases $\mathrm{Fe} / \mathrm{Cr}$ (001) multilayers were grown by molecular beam epitaxy (MBE), either Fe/Cr (001) superlattices for the Orsay team or $\mathrm{Fe} / \mathrm{Cr} / \mathrm{Fe}(001)$ trilayers for the Jülich group.

In these structures, when the configuration of the magnetizations in the neighboring Fe layers rotates from antiparallel to parallel alignment, a large resistance drop is observed, see figure 8. Since the reduction of the resistivity is significant, this effect has been called Giant Magnetoresistance or GMR.

The saturation field $\mathrm{H}_{S}$, is the field required to overcome the antiferromagnetic interlayer coupling between consecutive Fe layers and align their magnetizations. The magnetoresistance ratio is defined as the ratio of the resistivity change to the resistivity in the parallel configuration:

$$
M R(\%)=\frac{\rho_{A P}-\rho_{P}}{\rho_{P}} \cdot 100 \%
$$

The physical origin of GMR is found in the "two current model", demonstrating experimentally the theoretical model initially proposed by Mott in 1936 [40]. This model takes into account the influence of the electron spin on the

electronic transport in ferromagnetic conductors, as the spin splitting of the energy bands in the ferromagnetic state leads to specific transport behavior. 


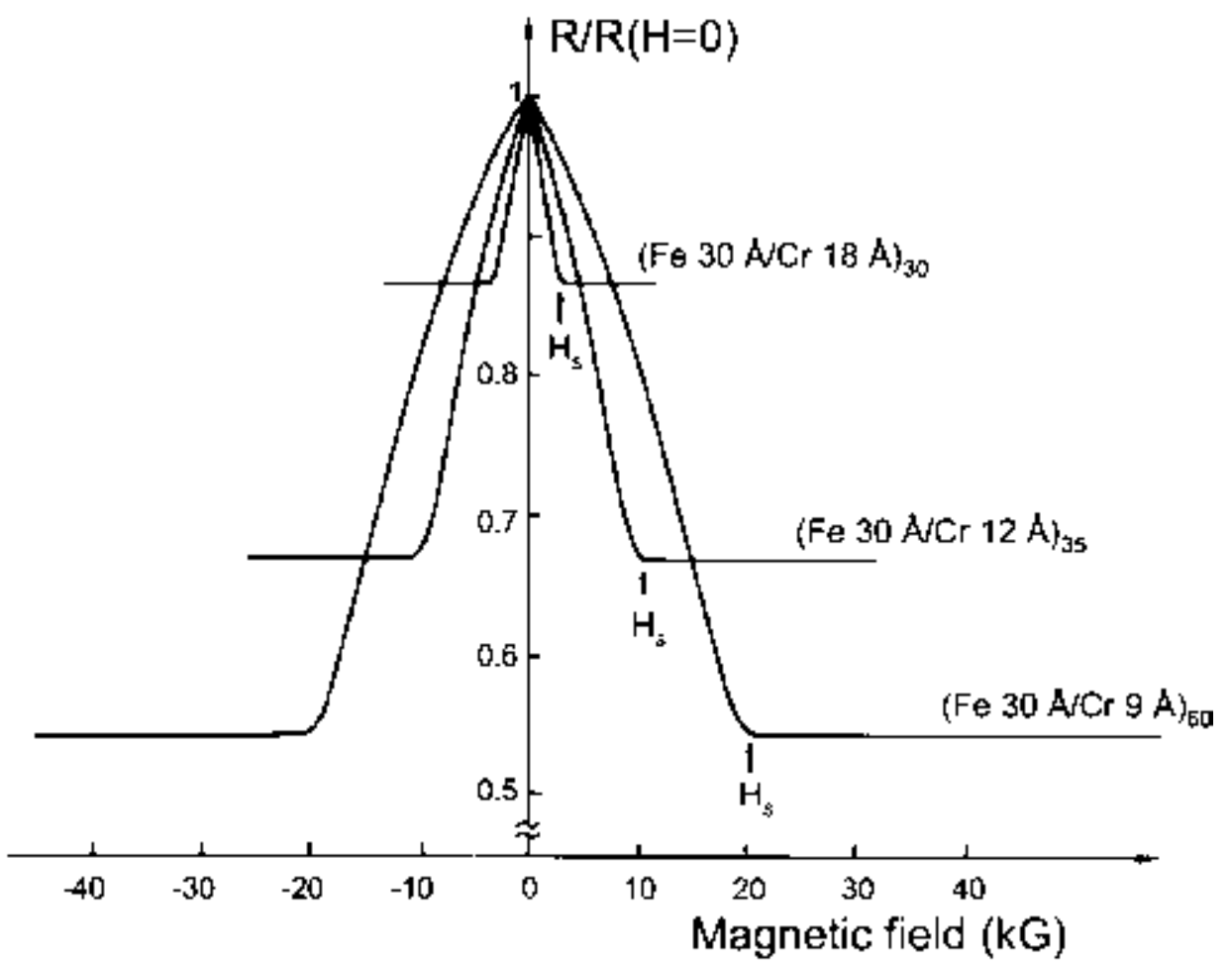

Figure 8: Variation of the resistance as a function of the magnetic field observed by Baibich et al. for $\mathrm{Fe} / \mathrm{Cr}$ superlattices at $4.2 \mathrm{~K} \mathrm{[2]}$.

Therefore, in the low temperature limit, when the spin flip scattering of the conduction electrons by magnons is frozen out, there are two independent parallel channels for the electrical current: spin $\uparrow$ (majority) and spin $\downarrow$ (minority) electrons, and the conductivity of the ferromagnet (FM) is the sum of two independent contributions. If $\rho_{\uparrow}\left(\rho_{\downarrow}\right)$ are the resistivities of the spin $\uparrow(\downarrow)$ channels, respectively, the resistivity can be expressed as:

$$
\rho=\frac{\rho_{\uparrow} \rho_{\downarrow}}{\rho_{\uparrow}+\rho_{\downarrow}}
$$

Electrons passing through the first FM become spin-polarized. If the nonmagnetic (NM) layer is thin enough, with thickness smaller than the electron mean free path for both spin directions, this polarization is maintained and interacts with the second FM, resulting in a different resistance depending on the relative orientation of the FM layers (see figure 9).

The most relevant GMR device is the spin valve [42]. Initially developed by Parkin at IBM, this system is composed by a "soft" ferromagnetic layer and a "pinned" ferromagnetic layer separated by a thin conductor layer. 

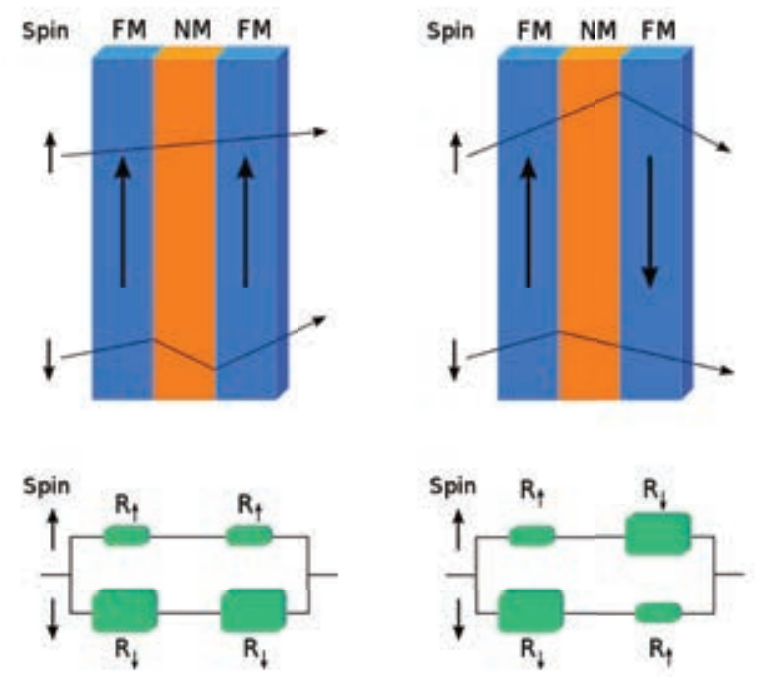

Figure 9: Schematics of spin dependent scattering for the explanation of the GMR effect [4].

Pinning is usually accomplished through exchange coupling with an adjacent antiferromagnetic layer. Therefore, the magnetization of the pinned electrode is fixed, while the magnetization direction of the other electrode can be changed by means of an external applied magnetic field. When the two ferromagnetic layers are parallel, conduction electrons pass between them more freely than when the magnetizations are anti-parallel, and thus the resistance is lower in the parallel magnetization case.

\section{Tunnel Magnetoresistance}

TMR is a magnetoresistive effect that occurs in magnetic tunnel junctions, i.e., heterostructures with two ferromagnetic electrodes separated by a thin insulator. Due to the spin asymmetry of density of state (DOS) in a ferromagnetic conductor, both the conduction and the tunneling probability through a potential barrier are spin-dependent properties, leading to a different resistance of the MTJ for the parallel and antiparallel magnetic configurations of their electrodes.

The first observation and interpretation of spin dependent tunneling between two ferromagnetic electrodes was reported by Jullière in 1975 [43], who obtained small TMR effects at low temperature (a variation of $14 \%$ at $4.2 \mathrm{~K}$ ) in a MTJ composed of an oxidized Ge semiconductor layer separating Co and Fe ferromagnetic electrodes. However, Jullière's observations were not easily reproducible and, actually, could not be really reproduced during 20 years. 
Jullière's model is widely used to account for the spin polarized tunneling effect. Thus, assuming that the tunneling process conserves the spin, the tunneling conductance can be considered as the sum of two independent conduction channels, one channel for each spin direction (Fig. 10). The relative variation of conductance and the DOS of each spin channel are then linked as follows in the Jullière formula:

$$
T M R=\frac{2 P_{1} P_{2}}{1-P_{1} P_{2}}
$$

where $P_{i}$ is the spin polarization of each electrode, defined as:

$$
P_{i}=\frac{D_{i \uparrow}-D_{i \downarrow}}{D_{i \uparrow}+D_{i \downarrow}}
$$

Being $D_{i \uparrow(\downarrow)}, \mathrm{i}=1,2$ the DOS of the two ferromagnetic electrodes at the Fermi level for the two spin directions.

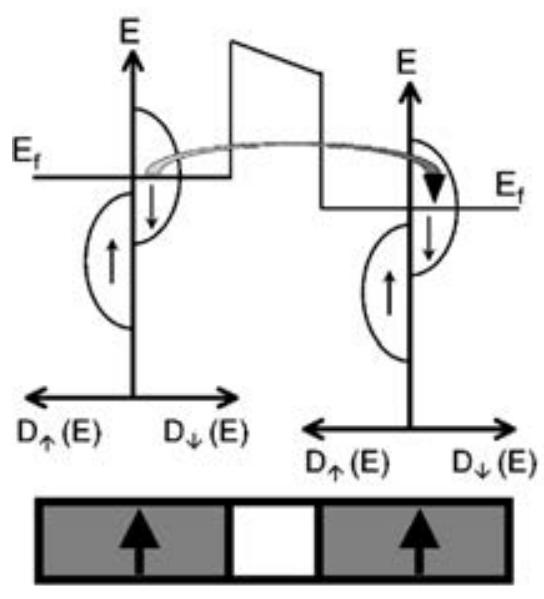

Parallel magnetizations

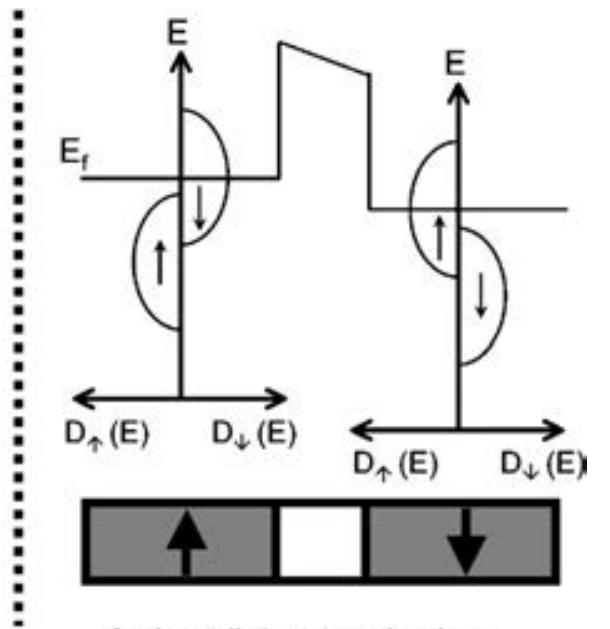

Antiparallel magnetizations

Figure 10: Schematic DOS for both magnetic electrodes in the parallel (right) and antiparallel (left) configurations of magnetizations. As the conductivity of each spin channel is proportional to both the spin DOS in the emitter and in the collector electrode, the conductivity of the barrier, which is the sum of the two channels conductivities, is strongly dependent on the magnetization configuration [44].

It was in 1995 that relatively large $(\approx 20 \%)$ and reproducible effects were obtained by Moodera's [11] and Miyazaki's [12] groups on MTJ with a tunnel barrier of amorphous alumina. One of the key parameters in these experiments was the growth of ultra thin tunneling barriers without any pinholes (metallic short circuits). Since then, an intense research effort has been devoted to the increase of the TMR ratio, using different materials as barrier and electrodes in MTJs (figure 11). 


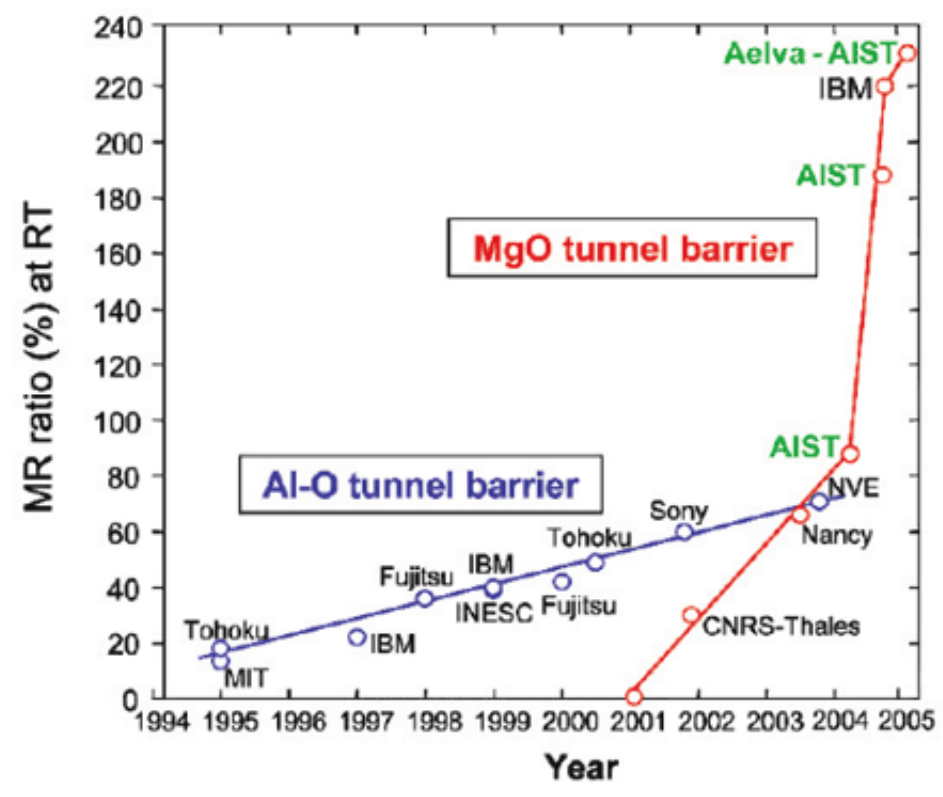

Figure 11: History of improvement in performance of MTJ devices (MR ratio) [45].

Following Jullière's model, a simple way to increase the TMR is to use electrodes with high spin polarization at the Fermi level. Half-metals are materials with the highest spin polarization: $|\mathrm{P}|=1$.

Regarding the tunnel barrier material, conventional MTJs with an amorphous aluminium oxide tunnel barrier $\left(\mathrm{Al}_{2} \mathrm{O}_{3}\right)$ have been extensively studied for device applications, exhibiting a magnetoresistance ratio up to $70 \%$ at room temperature. However, the most promising material is $\mathrm{MgO}$, with highest TMR ratios.

Yuasa reported a giant TMR ratio up to $180 \%$ at room temperature in single-crystal $\mathrm{Fe} / \mathrm{MgO} / \mathrm{Fe} \mathrm{MTJs}$ in 2004 [46], whose origin was found in a coherent spin-polarized tunnelling, where the symmetry of electron wave functions plays an important role. When the coherency of electron wave functions is conserved during tunnelling, only conduction electrons whose wave functions are totally symmetrical with respect to the barrier-normal axis are connected to the electronic states in the barrier region and have significant tunnelling probability. 


\section{Half metallic materials}

From equation 4, it is obvious that the TMR becomes infinite if $\mathrm{P}_{1}=\mathrm{P}_{2}=$ 1 , thus, if both electrodes have $100 \%$ spin polarization. This condition gets satisfied in the half metallic materials family.

Therefore, one of the main issues in the research field of spintronics is the production of magnetoresistive devices such as MTJs with half-metallic ferromagnetic materials at room temperature as active electrodes. A half-metal is any substance that acts as a conductor to electrons of one spin orientation, but as an insulator to those of the opposite orientation. In half-metals, the valence band related to one type of these electrons is fully filled and the other is partially filled. Therefore, only one type of electrons (either spin up or spin down) can pass through it. A schematic diagram of the band structure of a half-metal is shown below:

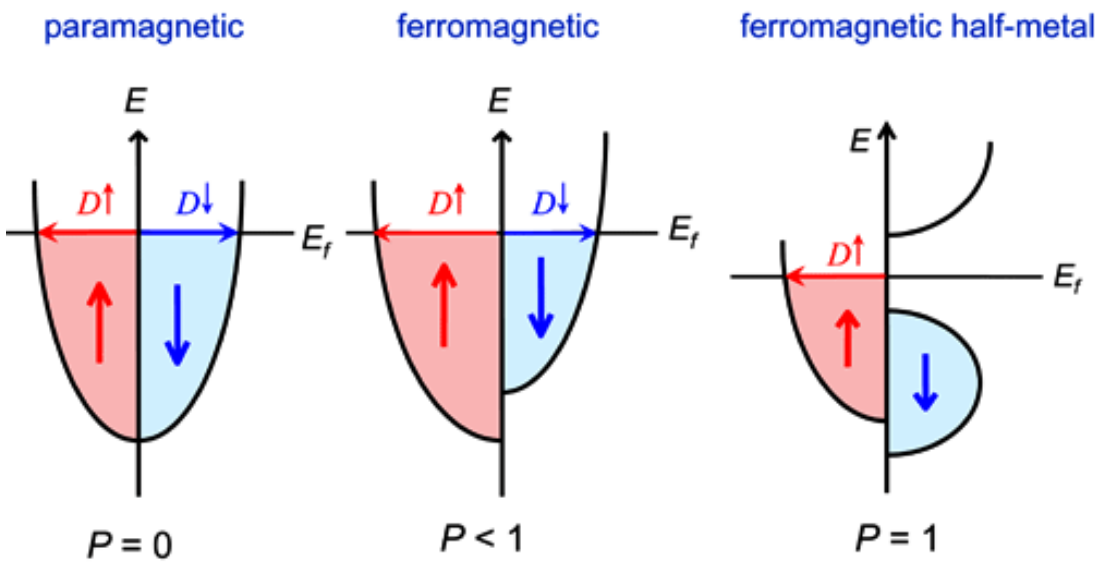

Figure 12: Density of states (DOS) of paramagnetic, ferromagnetic and ferromagnetic half-metal materials [47].

The first half-metallic ferromagnets were the half-Heusler alloys NiMnSb and PtMnSb, which were predicted by de Groot et al. [48] from electronic band structure calculations, but these materials are not suitable for spintronics applications because of difficulties in crystal growth. Subsequently, various half metallic ferromagnetic materials have been studied, such as oxides $\mathrm{CrO}_{2}$, $\mathrm{Fe}_{3} \mathrm{O}_{4}$, and (double) perovskites among others. The four types of predicted half-metals are shown in figure 13.

In order to obtain magnetoresistive devices operative at room temperature, materials with a high Curie temperature $\mathrm{T}_{C}$ are required. However, only a few oxides, semiconductors and metallic alloys are candidates to exhibit half metallicity [3]. 


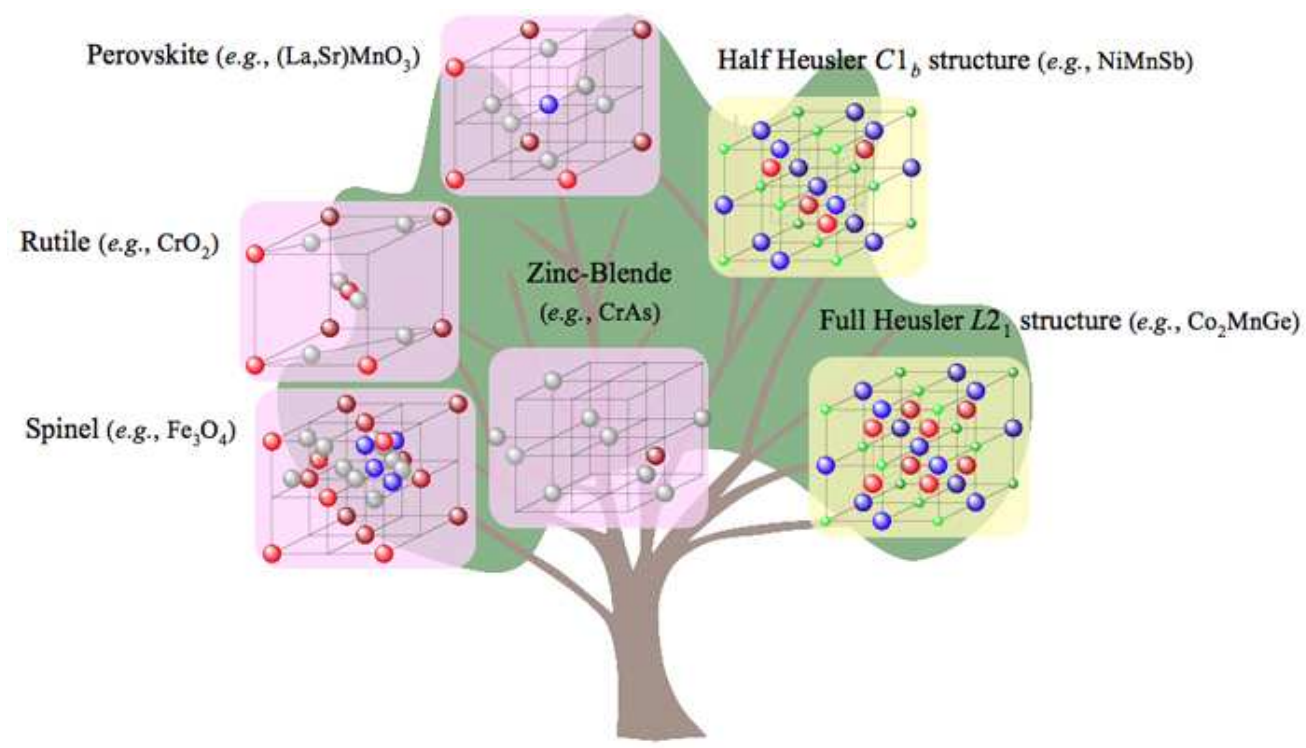

Figure 13: List of half-metallic ferromagnets [49].

In this thesis we have directed our efforts towards two promising materials to exhibit half-metallicity at room temperature: magnetite $\left(\mathrm{Fe}_{3} \mathrm{O}_{4}\right)$ and the double perovskite $\mathrm{Sr}_{2} \mathrm{CrReO}_{6}$, in chapters 2 and 3 , respectively, optimizing its growth conditions when deposited by PLD and performing a systematic study of their structural, morphological, magnetic and transport properties.

We have also studied the growth and properties of $L 1_{0}$-ordered FePt thin films. When this material is $L 1_{0}$-ordered, it presents a high magnetocrystalline anisotropy, which could solve the superparamagnetic limit problem when decreasing bit sizes in perpendicular data storage devices. Indeed, FePt has shown high tunnel magnetoresistance when being part of a MTJ with $\mathrm{MgO}(001)$ barrier [46, 50, 51].

Heterostructures with different electrodes materials and geometries have been studied in this thesis. In all the heterostructures the tunnel barrier is made of $\mathrm{MgO}$, as MTJs with an $\mathrm{MgO}$ barrier have shown the highest TMR ratios up to date. All the MTJs reported in literature have both electrodes with an in-plane easy magnetization direction or both perpendicular to the film surface, and TMR has never been examined in any MTJ including both in-plane and out-of-plane anisotropy geometries in MTJs electrodes. This is the reason why we decided to study $\mathrm{Fe}_{3} \mathrm{O}_{4} / \mathrm{MgO} / \mathrm{FePt}$ heterostructures, which represent an interesting and timely topic. 


\section{Chapter 1}

\section{Experimental Methods}

In this chapter, a brief overview of the main experimental techniques employed in this thesis for the growth and characterization of epitaxial thin films and multilayers is presented.

A large part of the growth and characterization process has been performed using the instruments available at the Institute of Nanoscience of Aragón (INA) and Institute of Material Science of Aragón (ICMA). A fruitful collaboration has been carried out with the IMEM-CNR research institute in Parma (Italy), resulting in the research presented in chapter 4 and part of chapter 5 . These results have been obtained through different techniques, the most relevant being described in this chapter.

\subsection{Epitaxial thin film growth systems}

Preparing very pure, high quality crystalline thin films requires very specialized deposition techniques, being pulsed laser deposition (PLD) and sputtering the film growth systems used in this thesis. Both of these techniques are physical vapor deposition processes in which a thin film of material is deposited onto a substrate according to the following sequence of steps:

- Evaporation

During this stage, a target consisting of the material to be deposited is bombarded by a high energy source such as a laser beam or a beam of electrons or ions, producing the vaporization of the target surface atoms. 
- Transportation

The vapor is transported from its source to the substrate.

This is a crucial part of the deposition from a technological point of view, as it will determine the uniformity of the coverage of the substrate.

- Reaction

In some cases it is necessary to introduce a background reactive gas such as oxygen, nitrogen or methane in order to obtain the desired stoichiometry of the film. Otherwise, the deposition can be carried out in high vacuum or in an inert gas atmosphere (e.g. Ar), and this step would not be part of the process.

- Deposition

The vapor undergoes condensation on the substrate to form the thin film. In this phase the condition of the substrate surface is essential.

In order to get high quality films, physical vapor deposition processes require the use of high vacuum chambers and, depending on the process, some reactions between target materials and the process gases may also take place at the substrate surface simultaneously with the deposition process. 


\subsubsection{Pulsed Laser Deposition}

This thesis is devoted to the study of films and heterostructures partially or completely grown by PLD, thus, this technique represents the essential tool necessary for the success of the work reported in the following chapters. PLD is a physical vapor deposition technique used to deposit high quality epitaxial films, which is conceptually and experimentally relatively simple.

The main advantage of this deposition technique is the preservation of the stoichiometry from the target material to the film, which allows the growth of complex oxide thin films. The principal disadvantage relies on its directionality, which restricts the film homogeneity to small areas. An effect that should also be controlled is "splashing", due to high energetic aggregates that can be produced during ablation and decrease the crystalline quality of the pulsed laser deposited films of certain materials. These issues will be discussed in the following pages.

Figure 1.1 shows a schematic diagram of an experimental PLD setup. A high-power laser is used as an external energy source to vaporize materials. The laser beam is focused and directed towards the vacuum process chamber by means of an external optical set, which usually consists of a lens, a mirror and also an attenuator may be used to decrease the laser energy down to the desired one.

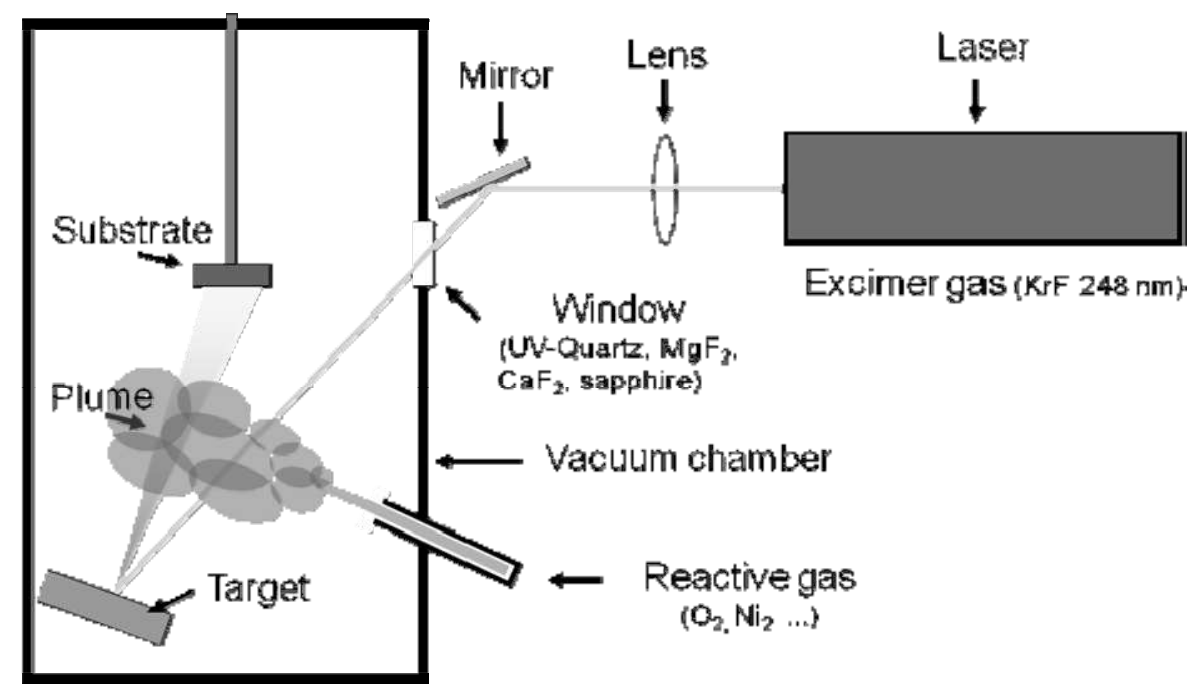

Figure 1.1: Schematic diagram of a Pulsed Laser Deposition setup. 
The laser beam enters the vacuum process chamber through a window made of a specific material, which must be transparent to the laser wavelength, e.g., quartz in case of an ultraviolet laser, and strikes the target, which is placed in a holder situated facing the substrate holder. The laser ablates the target surface producing a plasma called plume, which condensates on the chosen substrate producing the growth of the film.

\section{The history of Pulsed Laser Deposition}

After the first high-power ruby laser became available in 1960, a great amount of both theoretical and experimental studies were performed regarding the interactions of intense laser beam with solid surfaces [52] and gaseous materials. However, the laser-solid interaction is an extremely complex process and the formulation of a complete self-consistent model is a difficult task.

The simplest model was based on a thermal effect which assumed a delay between the laser absorption and vaporization [53], determined by the thermal diffusion rate until a critical temperature in the solid is reached. However, this model does not work properly for beam-solid interactions at power densities above $10^{8} \mathrm{~W} / \mathrm{cm}^{2}$, since overestimates the surface temperature during the interaction and, in addition, neglects the interaction beween the laser radiation and the plasma of the plume. Although more realistic models have been developed, none of them account for all the observations relating the laser-target interaction.

The easy vaporization of different materials by means of intense laser radiation suggested that this could be used to deposit thin films, which was demonstrated in 1965, when Smith and Turner utilized a ruby laser to deposit the first thin films [54]. The advances achieved in laser technology provided other types of laser which were used in PLD successfully, such as the $\mathrm{CO}_{2}$ lasers and the Nd:YAG lasers, with a higher repetition rate than the ruby lasers, making the growth of thicker films possible. Although the stoichiometric preservation of a multiple component target to the growing film was demonstrated, the quality of the deposited layers was still inferior to that obtained in films grown with other techniques such as CVD and MBE.

During the 1970s, the development of electronic switches for generating shorter optical pulses made the obtention of high peak power (more than $10^{8}$ $\mathrm{W} / \mathrm{cm}^{2}$ ) possible, and the achieved shorter wavelength radiation produced shallower absorption depth, reduced splashing, and smaller heated volume, which improved the congruent evaporation. 
In the early 1980s, a few research groups achieved remarkable results when manufacturing thin film structures utilizing laser technology, mostly used as optical coatings. An improvement in crystallinity over electron-beam-deposited films was observed and attributed to the high surface mobility due to hyperthermal impact energy 55]. But the breakthrough came in 1987 when Dijkkamp and Venkatesan [56] were able to laser deposit a thin film of the high temperature superconductor $\mathrm{YBa}_{2} \mathrm{Cu}_{3} \mathrm{O}_{7}$ with much higher quality than films deposited with alternative techniques.

Since then, the technique of Pulsed Laser Deposition has been successfully utilized to fabricate high quality crystalline films and the deposition of ceramic oxides, nitride films, metallic multilayers and various superlattices has been carried out. Finally, it was in the 1990s where PLD became a very competitive tool for the growth of thin and well defined films with complex stoichiometry thanks to the development of new laser technology, such as lasers with high repetition rate and short pulse durations.

Pulsed Laser Deposition technique has also been applied to metal film deposition, production of coatings, biocompatible films, and even polymer films, resulting in an exponential increase of published articles during the last years, as can be observed in figure 1.2. However, this technique has two important drawbacks: the "splashing" phenomenon, which decreases film quality and will be further explained in next subsection, and the lack of film uniformity over a large area due to the high directionality of the plume, restricting possible PLD industrial applications.

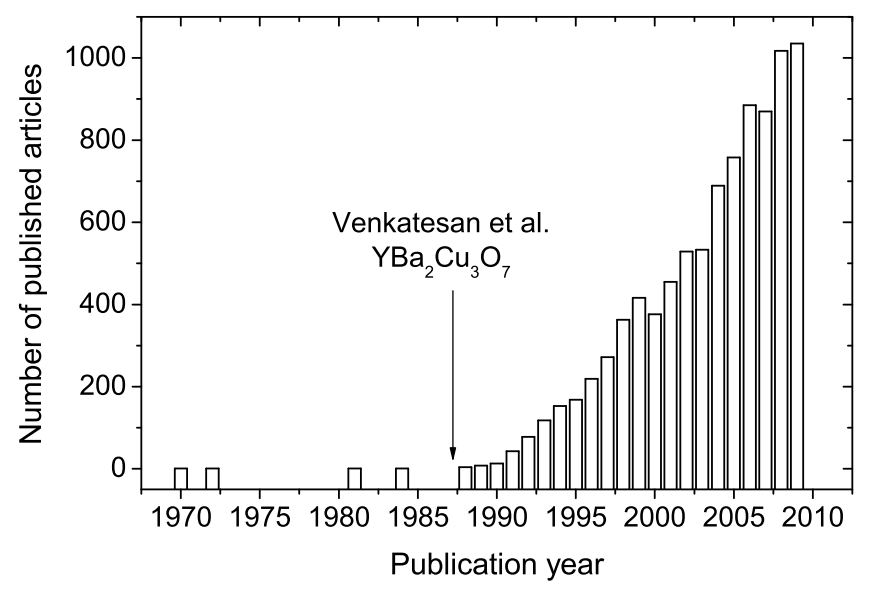

Figure 1.2: Evolution of publications concerning Pulsed Laser Deposition in the last 30 years. The information has been compiled from ISI Web of Knowledge database. 


\section{Stages of the PLD process}

The process of PLD can generally be divided into four stages, being all of them crucial for the crystallinity, uniformity and stoichiometry of the resulting film.

\section{Laser ablation of the target material and creation of a plasma :}

The ablation of the target material upon laser irradiation and the creation of plasma are very complex processes. The removal of atoms from the bulk material is done by vaporization at the surface region in a state of non-equilibrium where the incident laser pulse penetrates into the surface of the material within the penetration depth. This dimension is dependent on the laser wavelength and the refraction index of the target material at the chosen laser wavelength, and is typically around $10 \mathrm{~nm}$ for most materials.

The ablation mechanism depends on laser flux density and pulse duration, as well as the optical, topological and thermodynamical properties of the target, and can be enhanced by microscopic cracks, voids, and nodules at its surface. The basic thermal cycle induced by laser pulses can be observed in figure 1.3. When the laser pulse is absorbed by the target, melting and vaporization begin, melt front propagates into the solid and then recedes, allowing the resolidification of the material. When the solidification completes, frozen waves alter surface topography, which will interact with the next laser pulse.

Although it is a complex process, the materials release from the target surface is essentially stoichiometric, a great advantage of the PLD deposition technique. However, if the absorption depth is too high, thick liquid layers are produced, which can cause splashing of micron-sized particulates (droplets) and deviation from congruent evaporation.

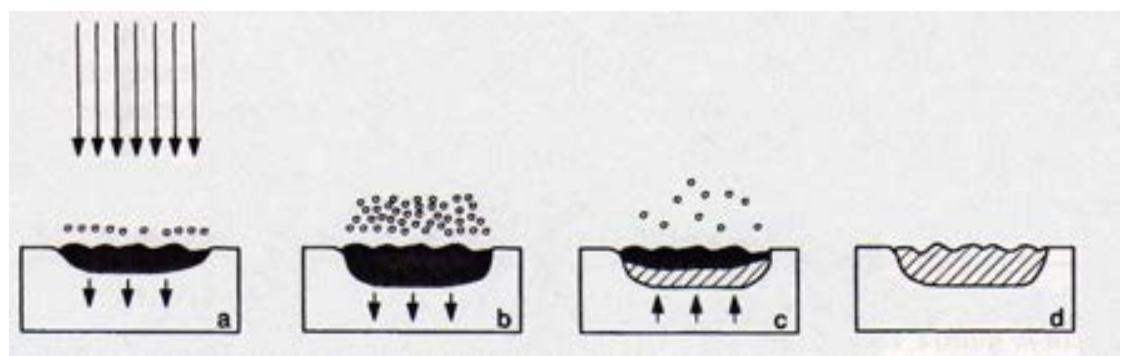

Figure 1.3: Schematic of the basic thermal cycle induced by a laser pulse [20]. (a) Laser pulse is absorbed and the vaporization begins (shaded areas indicates melted material, circles the vaporized particles, and arrows the motion of the solid-liquid interface). (b) Melt front propagates into the solid. (c) Melt front recedes (crosshatched area indicates resolidified material). (d) Solidification completes. 
Splashing becomes particularly problematic for electronic devices quality, where the particulates can induce the formation of defects that lower carrier mobility, this being considered one of the two main drawbacks of PLD. This occurs in most materials except for those with high vapor pressure at a temperature much below the melting temperature (such as CdTe and most II-VI compounds), dense and single-crystal targets, and targets with high thermal conductivity [20].

The main mechanisms that can lead to droplet formation are:

- Subsurface Boiling: This occurs when the time required to transfer laser energy into heat is short, producing the superheating of a subsurface layer before the surface itself has reached the vapor phase, and, therefore, the emission of micron-sized molten globules that impact on the substrate. This effect can be reduced by decreasing the laser power. The maximum laser power density $\left(D_{\max }\right)$ that a solid surface can absorb without causing splashing was estimated by H. Schwartz [57]:

$$
D_{\max }=\frac{L \rho H_{e v}}{t_{r}}
$$

where $t_{r}$ is the relaxation time, $\mathrm{L}$ is the range of surface penetration of the light density into the solid with density $\rho$ and heat evaporation $H_{e v}$.

- The surface liquid layer can be expulsed by the shock wave recoil pressure of the plume. This effect is indistinguishable from the subsurface boiling, and can also be reduced by lowering the laser power density.

- Exfoliation: In this case, the solid particulates ejected from the target are randomly shaped. The repetitive laser ablation produce the target surface erosion, forming long needle-shaped microstructures of few microns. These structures are mechanically fragile and can break due to the thermal shock induced during the laser radiation [58], producing loose particulates that are carried toward the substrate by the expanding plume and condense onto the thin film. This effect could be minimized by a periodic polishing of the target, in order to maintain a smooth surface.

The splashing of droplets on the substrate surface can be decreased with an "off-axis" laser-ablation geometry, as, while particulates travel along their initial trajectory directions, the light species (ablated ions) undergo scattering, modifying their trajectories. Therefore, by adjusting the substrate holder position, the deposition of droplets can be avoided. 


\section{Plume dynamics :}

The ejected species, including neutral atoms, ions, electrons, molecules, clusters, micron-sized solid particulates and molten globules, form a laserproduced plasma plume that expands rapidly away from the target surface (velocities typically $\sim 10^{6} \mathrm{~cm} / \mathrm{s}$ in vacuum), parallel to the normal vector of the target surface towards the substrate.

The plume is highly energetic and very directional, although it can be attenuated and thermalized by ambient gases during PLD, changing its spatial distribution, the film deposition rate and the kinetic energy distribution of the deposition species. The background gas effect on the plume shape is different depending on pressure, and can be described in three stages:

- In vacuum almost no scattering occurs with the background gas and the plume is very narrow and forward directed.

- With an intermediate background pressure a splitting of the high energetic ions from the less energetic species can be observed.

- High pressures produce a more diffusion-like expansion of the ablated material, which depends on the background gas mass and can influence the stoichiometry of the deposited film.

In the plume, different component species have different angular distributions, associated with differences in species charge and mass and described by $\cos ^{n} \theta$ law with a shape similar to a Gaussian curve, where $\mathrm{n}$ takes typical values between 8 and 12 (see figure 1.4). This effect may lead to angular stoichiometric deviations in the film for wide surfaces.

\section{Deposition of the ablated material on the substrate :}

The third stage is important to determine the quality of the deposited films.

It is known that the kinetic energy of the impinging species in PLD typically lie in the 1-100 eV range [20, 59]. The energetic species ablated from the target strike substrate surface producing sputtering of species from the substrate, which form a collision region with the particles emitted from the target. If the condensation rate is high enough, a thermodynamic equilibrium is reached, where the film grows directly from the particles emitted from the target. 
However, it has been shown that particles with kinetic energies around $50 \mathrm{eV}$ can resputter the film already deposited on the substrate, causing damage to the surface and also producing defect formation in the deposited film. This can be overcome by decreasing the laser power density.
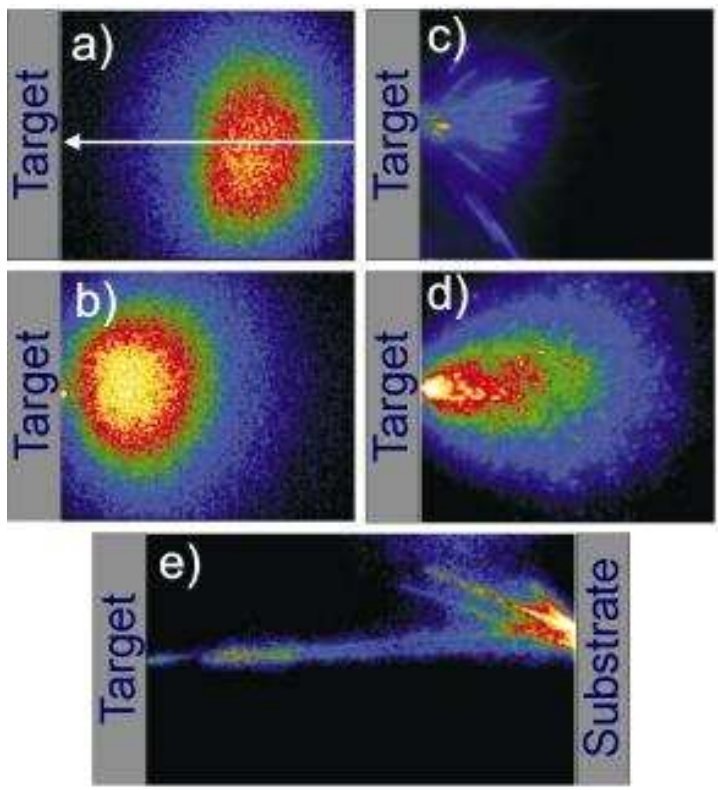

Figure 1.4: A selection of intensified Charge Coupled Device (i-CCD) images of the emitting species arising in $248 \mathrm{~nm}$ nanosecond pulsed laser ablation of a graphite target in vacuum: (a) $\mathrm{C}^{+}$ions (b) $\mathrm{C}$ neutrals (c)-(e) particulates. (a), (b) and (d) are accumulated images of 200 laser pulses, while (c) and (e) are single pulse events 60, 61].

\section{Nucleation and growth of the film onto the substrate surface :}

The nucleation process and growth kinetics of the film depend on several growth parameters including [62]:

- Laser Parameters

Film quality, stoichiometry [63] and deposition flux are affected by the laser wavelength, fluence, energy and repetition frequency of the laser pulses, as well as the ionization degree of the ablated material. Generally, the nucleation density increases when the deposition flux is increased.

- Substrate temperature

Usually, the nucleation density decreases as the temperature is increased. 
- Substrate surface

The nucleation and growth can be affected by the surface preparation, the miscut of the substrate, as well as the roughness of the substrate.

- Background Pressure

If the film grows off stoichiometry, this will affect the nucleation density and film quality. Together with the substrate temperature and surface morphology, background pressure determines the film growth and microstructure.

- Geometry-related parameters such as the target-substrate distance and the spot size influence the film thickness and the energy of the ablated particles, and, therefore, the homogeneity of the film thickness on the substrate.

In PLD the nucleation density is large compared with Molecular Beam Epitaxy or Sputtering Deposition, which increases the smoothness of the deposited film. Depending on the deposition parameters above, three growth modes are possible: three dimensional island growth (VolmerWeber), two dimensional full monolayer growth (Frank-van der Merwe), and two dimensional growth of full monolayers followed by nucleation and growth of three dimensional islands (Stranski-Krastanov), already detailed in the introduction of this thesis. 


\section{Pulsed Laser Deposition equipment}

PLD is a versatile technique for preparing thin films and multilayer structures. The basic equipment required can be reduced to:

- Lasers

In general, the useful range of laser wavelengths for thin film growth by PLD is found between $200 \mathrm{~nm}$ and $400 \mathrm{~nm}$, as most materials used for deposition exhibit a strong absorption in this spectral range. Most of the work performed with PLD has been made with $\mathrm{Nd}^{3+}$ :YAG solid-state lasers and gas excimer lasers. The excimer is generally the laser chosen for PLD, as can achieve pulse repetition rates up to several hundred hertz with typical energies of $500 \mathrm{~mJ} /$ pulse.

The excimer molecules are formed in a gaseous mixture of their component gases, such as $K r$ and $F_{2}$ in the case of the KrF laser. Energy is pumped into the gas mixture through avalanche electric discharge excitation, creating ionic and electronically excited species that react chemically and produce the excimer molecules. Once the excimer is formed, it decays, with a lifetime of $\sim 2.5 \mathrm{~ns}$. Some of the reactions leading to the formation of the excimer molecules for the case of $\mathrm{KrF}$ are listed below, where ${ }^{*}$ denotes electronically excited species:

$$
\begin{aligned}
K r+e^{-} & \longrightarrow K r^{+}, K r^{*}, K r_{2}^{+} \\
F_{2}+e^{-} & \longrightarrow F+F^{-} \\
K r^{+}+F^{-}+X & \longrightarrow K r F^{*}+X \\
K r_{2}^{+}+F^{-} & \longrightarrow K r F^{*}+K r \\
K r^{*}+F_{2}+X & \longrightarrow K r F^{*}+F
\end{aligned}
$$

As homogeneous uniform laser output is necessary in order to obtain good quality deposition, hot spots and deviations from uniformity should be avoided. This issue is especially important when working with multicomponent deposition target, where poor beam quality could result in non stoichiometric films and droplet formation. Figure 1.5 display the typical three dimensional beam profile of our TuiLaser $\mathrm{KrF} 248 \mathrm{~nm}$ laser.

\section{- Optics}

In order to prepare adequately the laser beam, optical elements such as lenses, apertures, mirrors, beam splitters and attenuators are placed between the output port of the laser and the entrance window to the deposition chamber. The optical external elements, as well as the deposition chamber entrance window, must be perfectly clean and made of different materials specifically designed for the used laser wavelength. 


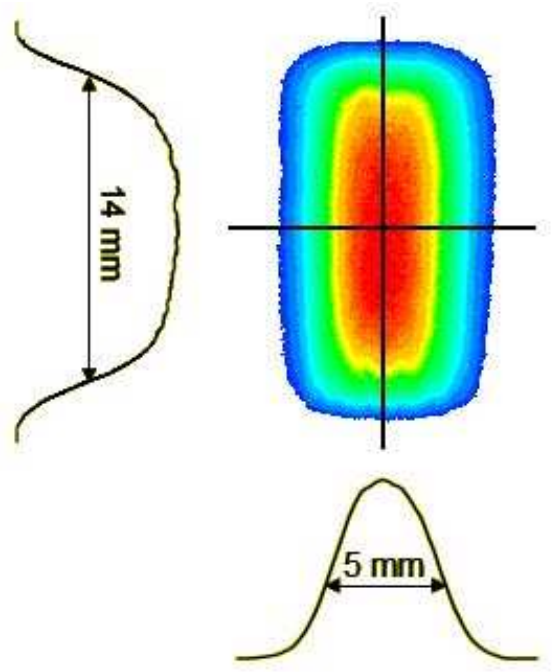

Figure 1.5: Intensity beam profile, obtained from our TuiLaser KrF $248 \mathrm{~nm}$ laser.

- Deposition system

Once the laser beam passes through the optical elements, it enters the deposition system. A typical deposition system consists of a vacuum chamber in which the substrate holder, substrate heater, target manipulation system, gas flow system, pumps and vacuum gauging are situated.

The Pulsed Laser Deposition system used in this thesis can be seen in figure 1.6. It is a combined PLD-Sputtering system by Neocera Inc. company located at the INA, with a $70 \mathrm{l}$ ultra-high vacuum chamber. As there is a loadlock chamber, it is not necessary to break the vacuum each time a substrate is inserted or a sample removed to and from the process chamber, respectively. The turbo-molecular pump connected to the loadlock chamber can provide a pressure as low as $\sim 10^{-7}$ Torr either only in the loadlock compartment or in all the system. The cryogenic pump attached to the process chamber can decrease the background pressure down to $5 \times 10^{-9}$ Torr.

The chamber can be filled with e.g. oxygen, nitrogen or argon, the pressure of which can be tuned directly with mass-flow controllers at the gas entrances, with the turbo molecular pump rotation speed or with the butterfly valve located at the cryogenic pump entrance.

The substrate holder can be placed facing either the PLD or the sputtering targets depending on the deposition technique desired, and it can also perform a continuous rotation. With the oxygen-compatible heater located at the substrate holder positioning system, temperatures as high as $850^{\circ} \mathrm{C}$ can be reached. 


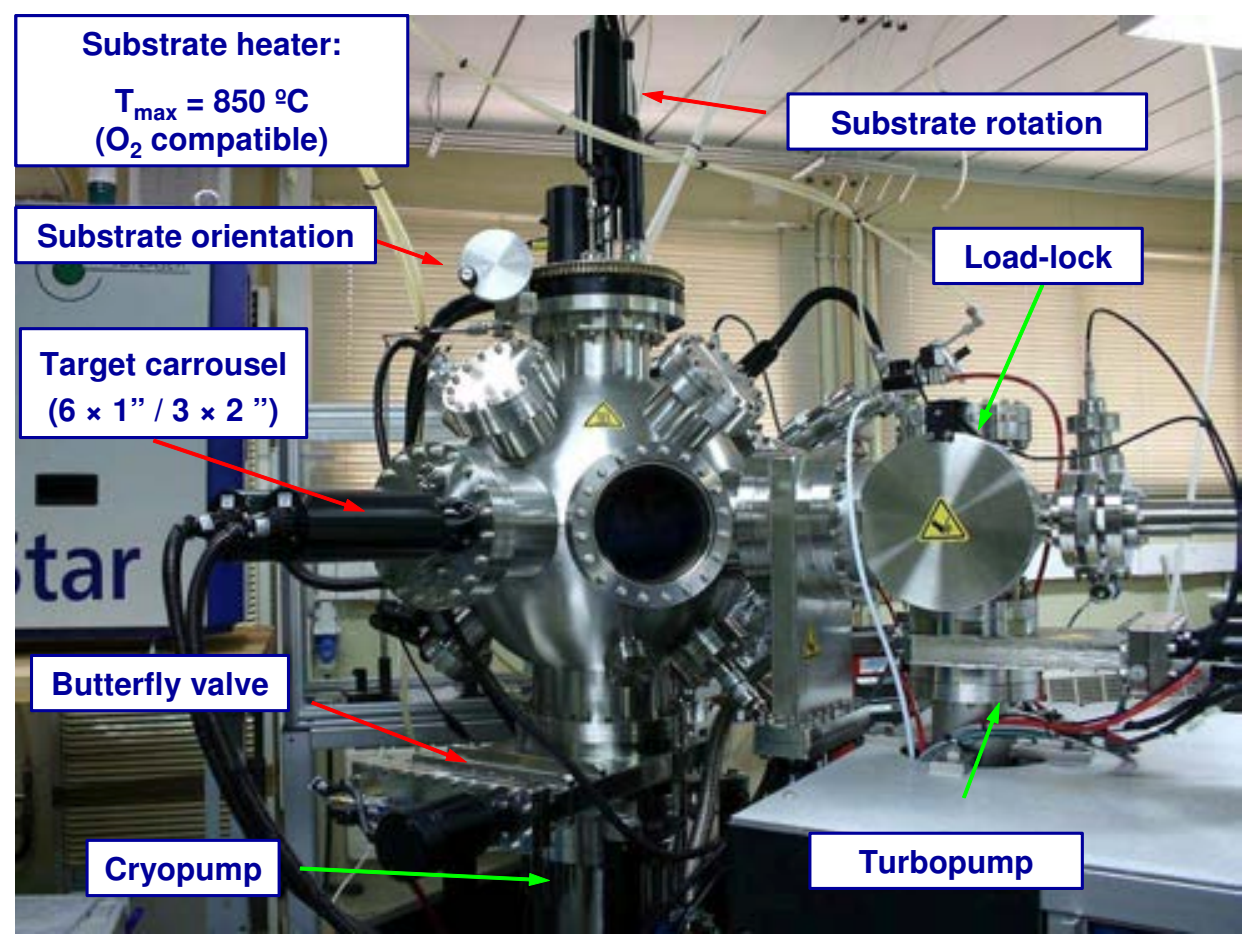

Figure 1.6: Combined PLD-Sputtering system by Neocera Inc. company.

The sinterized or commercial targets are attached to a support called target carrousel, which, in our PLD system, can hold either 6 targets with 1 inch in diameter or 3 targets with 2 inches in diameter. The targets can perform a rotational motion and translational up and down movement along its axis, in order to homogenize their surface erosion. This configuration also permits the deposition of films composed by layers of different materials, due to the multi-target carrousel.

The optical system consists of an aperture, a lens and a mirror which direct and focalize the laser beam coming from the $\mathrm{KrF}$ excimer laser from TuiLaser Company. This laser has a wavelength of $248 \mathrm{~nm}, 250 \mathrm{~mJ}$ maximum energy per pulse and a beam size of $1 \mathrm{~cm} \times 1.5 \mathrm{~cm}$ at the laser exit door, which is focused down to a $3 \mathrm{~mm} \times 4 \mathrm{~mm}$ spot at the target surface by means of the specific optical system. 


\subsubsection{Sputtering}

Sputtering is a physical vapor deposition technique in which ions accelerated to energies of a few $\mathrm{keV}$ by a potential difference are directed towards a target surface. When the striking ions overcome the binding energy of the target atoms, these are ejected from the target and transported to the substrate, where the sputtered atoms form a film.

In principle, any kind of high-energy particles such as electrons, neutrons or photons can sputter matter. However, the momentum transfer between particles is more efficient between similar-mass particles, and hence, accelerated ions are more suitable and usually used as the high-energy particles.

Sputtering has become one of the most widely used techniques for depositing various metallic or oxide films on wafers, due to the advantages that it offers over other physical vapor deposition methods:

- Sputtering can be performed on large-size substrates, allowing the deposition of thin films with uniform thickness over large areas.

- Film thickness is easily controlled by fixing the operating parameters and simply adjusting the deposition time and electrical power.

- Nearly any material can be sputtered, including compounds where one component may have a much larger vapor pressure than the others.

- Control of the alloy composition, as well as other film properties such as step coverage and grain structure are more easily accomplished than by evaporation-assisted deposition, due to the higher kinetic energies of the arriving atoms or molecules.

- Ion bombardment of the substrate often enhances adhesion of the film.

- Sputter of the substrate in vacuum prior to film deposition can be done in order to clean the substrate surface.

Sputtering, however, has some disadvantages compared to evaporation:

- Sputtering typically operates in a poorer vacuum range than deposition by evaporation, having a greater tendency to introduce impurities in both the substrate and the film. Therefore, the inert gas must be extremely pure with a consequent increase in price.

- Sputtered films always contain inclusion of the inert sputtering gas.

- The substrate can be damaged by the ion-bombardment, as well as the growing film. 
- Some materials such as organic solids are easily degraded by ionic bombardment.

The sputtering processes in thin film growth can be grouped in two main types: ion-beam sputtering, where ion sources provide ion beams that are accelerated, collimated and directed towards the target, and discharge sputtering, in which two facing electrodes produce the voltage necessary to ionize a background gas, whose ions sputter the target surface. Depending on the discharge source, discharge sputtering can be mainly divided in Direct Current (DC) and Radio Frequency (RF) Sputtering, and if a reactive gas is introduced in the process chamber, reactive sputtering. Another improvement in the gun design is the magnetron, where a permanent magnet array is incorporated, trapping secondary electrons close to the target surface. This variation of sputtering, called magnetron sputtering, can be applied to both DC and RF discharge techniques, and provides a higher ion density in the region close to the target, increasing the deposition rate and allowing that the plasma be sustained at a lower pressure. However, and due to the magnetic field, the target region where sputtering takes place is limited, producing a waste of most of the target material, with the consequent increase in cost.

\section{DC-Glow Discharge Sputtering}

DC glow discharge sputtering is one of the simplest sputtering techniques. However, non-conductive targets cannot be sputtered by this technique.

A schematic of a DC-glow discharge sputtering system is displayed in figure 1.7. An inert gas (generally Ar), present inside a vacuum chamber, is ionized by an electric field applied between the chosen target (cathode) and the substrate (anode) placed oppositely. The positive ions in the plasma are accelerated to energies of a few $\mathrm{keV}$ by the potential difference towards the target surface, and when the striking cations overcome the binding energy of the target atoms, these are ejected from the target and transported to the substrate through a region of reduced pressure, forming the thin film.

When a dc power is applied to the target, different stages in the process can be distinguished, as shown in figure 1.8. Initially, a very small current flows due to the limited number of charge carriers in the system, and as the applied voltage increases, more charge carriers are created from the secondary electrons emitted from the target and from impact ionization. At this stage, by applying a constant voltage $V_{B}$ (Breakdown Voltage) the current increases and a "Towsend discharge" is created [64]. The avalanche begins leading to a self-sustaining plasma, the "normal glow" state. 
Finally, the gas glow becomes visible and a rise in the current and drop in voltage appear in the "abnormal glow" state, where the sputtering takes place.

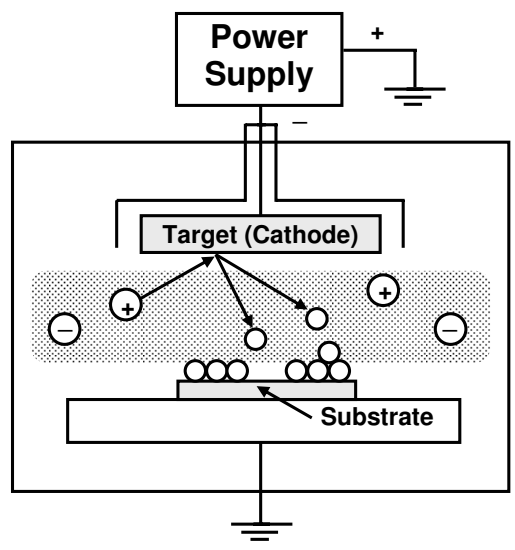

Figure 1.7: Schematic of a dc glow discharge sputtering system.

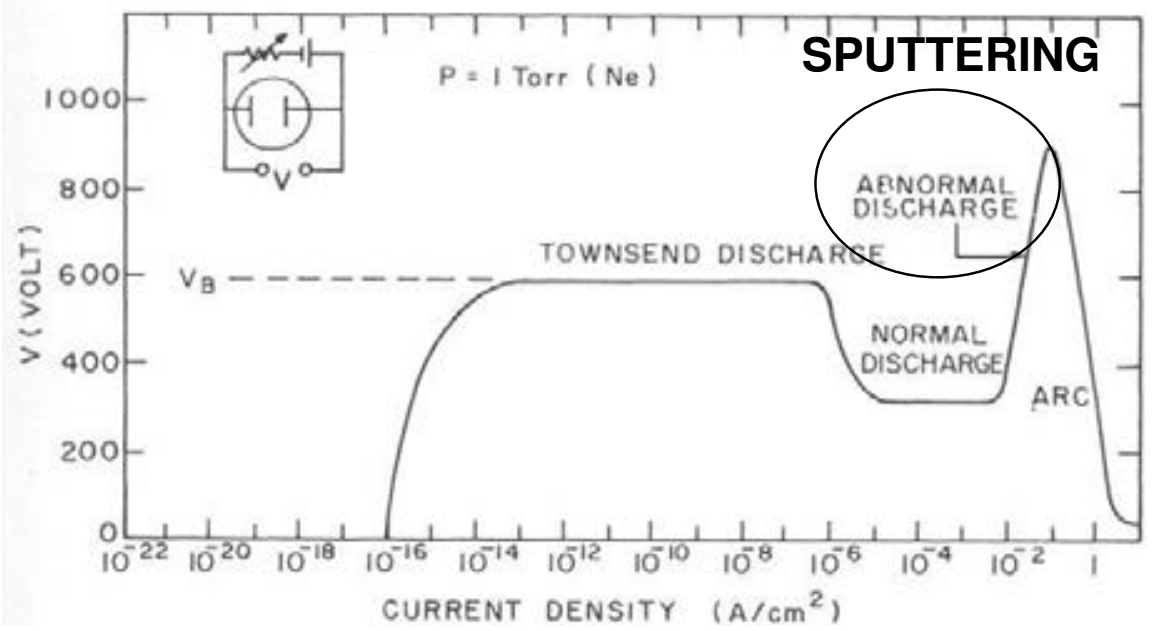

Figure 1.8: Voltage distribution and discharge characteristics across dc glow discharge [64].

The typical discharge voltage applied at the target is $\sim 500-5000 \mathrm{~V}$. The sputtering rate can be increased by increasing the discharge voltage and by increasing the inert gas pressure. However, if the pressure is increased substantially, the ions are slowed down due to the inelastic collisions with the gas atoms, decreasing the sputtering rate [65]. Finally, the deposition rate can be increased at reduced pressures by means of a magnetron sputtering, thanks to the higher efficiency in the ionization process achieved by means of the magnetic field at the target surface. 
The sputtering system employed in this thesis is part of the combined PLDsputtering system by Neocera Inc. company already described in section 1.1.1. The sputtering module of the system (shown in figure 1.9) has three watercooled targets, two of them adapted for magnetic material deposition by means of stronger magnetrons. At present, there are three power sources available: two of them DC, and the third one is an RF source.

This sputtering has been used for the deposition of $\mathrm{Au}$ films on some of the films studied in this thesis, in order to avoid contamination or oxidation of the upper metallic film, in the case of heterostructures. A constant pure argon (99.9999\%) pressure is maintained by fixing the flux of gas through a mass flow controller with a certain opening of the butterfly valve to control the cryopump speed pumping (see figure 1.6).

The substrate positioning and heating system is the same of the PLD system, therefore, the substrate can be faced towards the sputtering guns, rotated at up to $60 \mathrm{deg} / \mathrm{sec}$ during the sputtering process in order to obtain films with uniform thickness, and heated up to $850^{\circ} \mathrm{C}$.

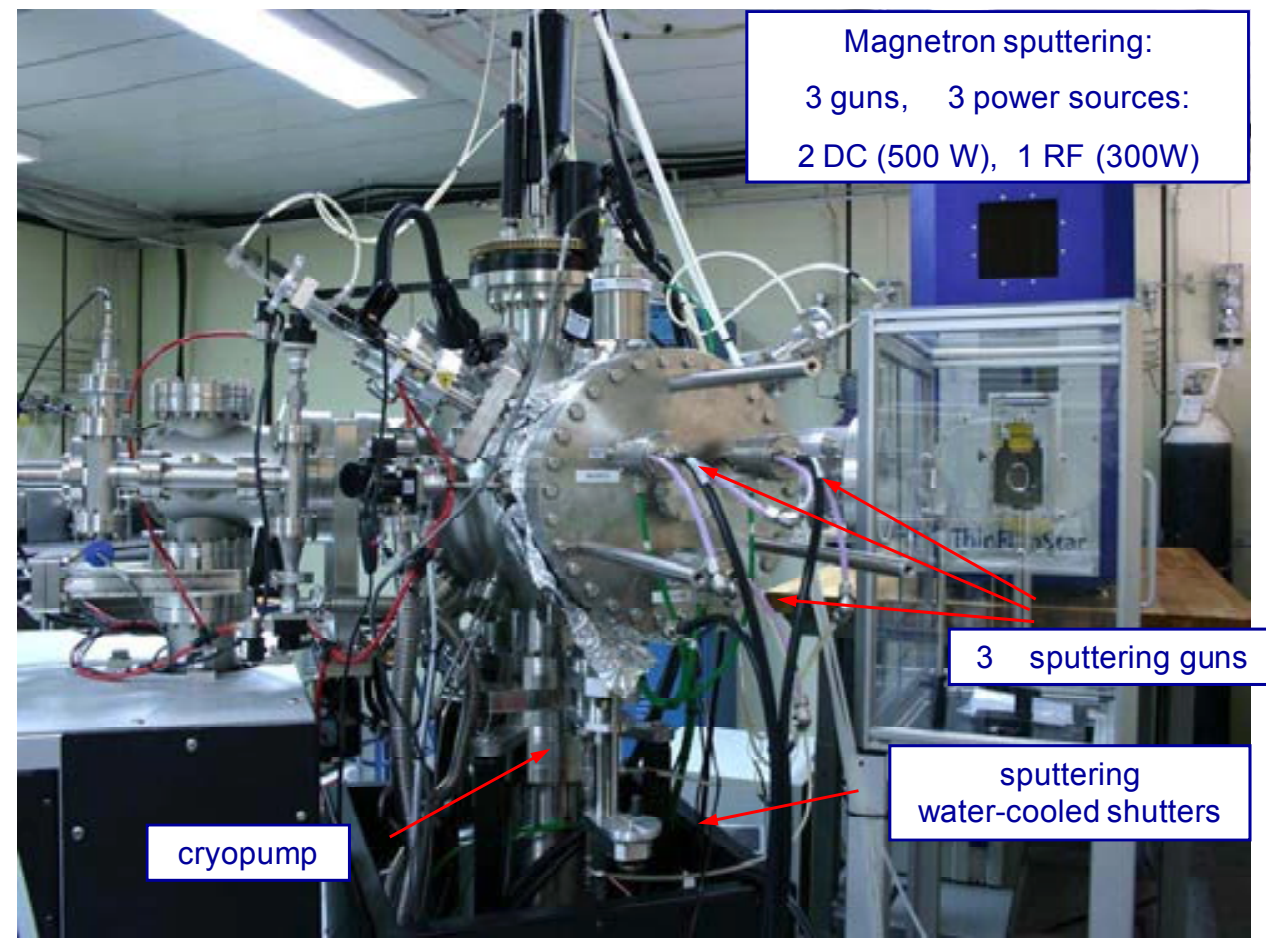

Figure 1.9: Combined PLD-Sputtering system by Neocera Inc. company, sputtering view. 


\section{RF Sputtering}

The charge problem when trying to sputter insulating targets can be avoided with the use of RF sputtering, where the sign of the anode-cathode potential difference is varied at a high rate, allowing the dissipation of the accumulated charge. Therefore, although RF sputtering maintains the fundamentals of DC discharge, it is specially suitable for dielectric film deposition and reactive sputtering, and the higher efficiency in the striking of the discharge allows lower process pressures, with the consequent lower possibility of film contamination.

The RF Sputtering system employed in chapter 4 and part of chapter 5 is located at the IMEM-CNR research institute in Parma (Italy)(see figure 1.10). It is constituted by a load-lock and a main chamber, where three targets set in vertical position can be simultaneously sputtered, as each of them has its own RF power supply operating at $13.56 \mathrm{MHz}$. In the load-lock a vacuum of $7.5 \times$ $10^{-6}$ Torr is made by a turbomolecular pump, whereas a vacuum around 2.3 $3.8 \times 10^{-8}$ Torr can be reached in the main chamber through a cryopump. The main chamber is separated from the cryopump by a throttle valve that can be partially closed allowing to operate in clean conditions during the sputtering process, but also avoiding the saturation of the cryopump by the sputtering gas.

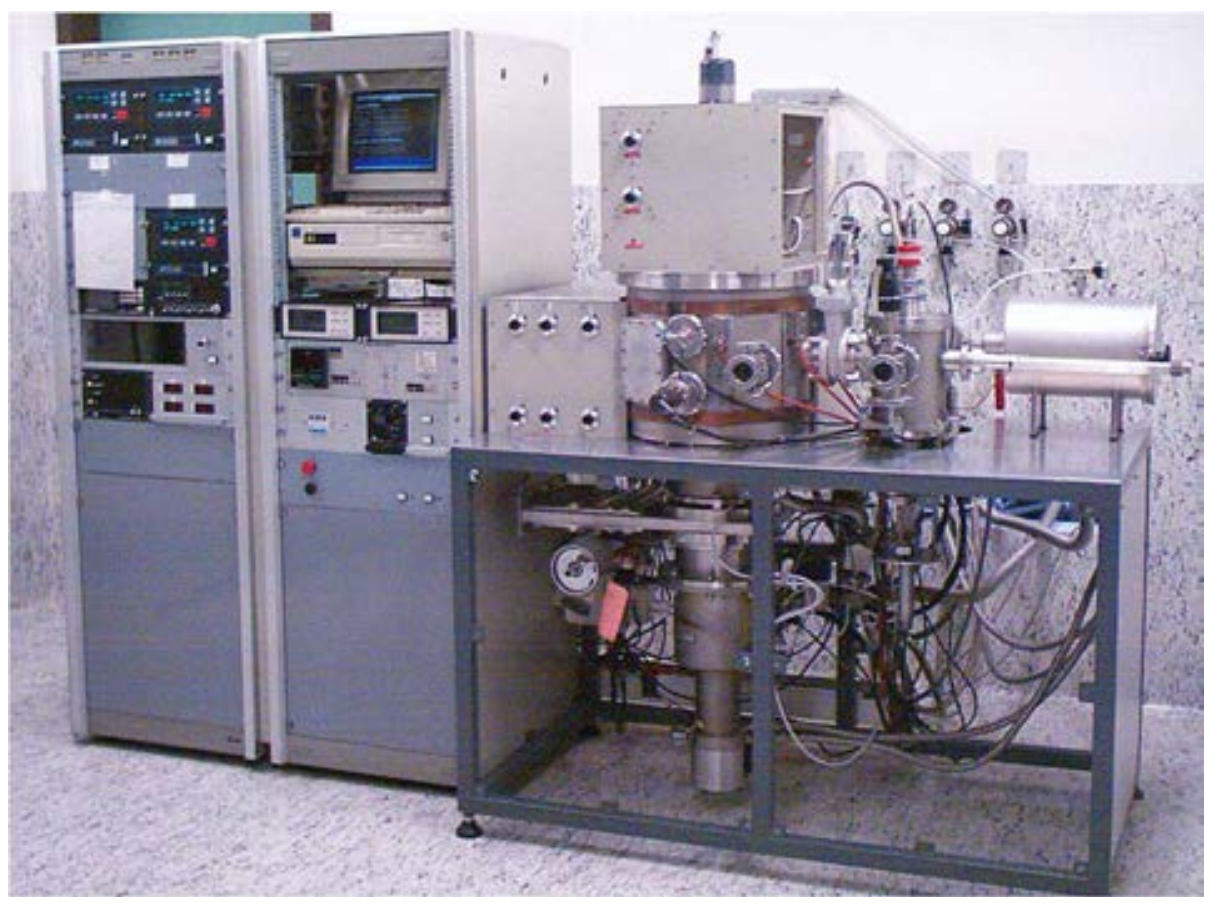

Figure 1.10: RF-Sputtering system available at the IMEM-CNR research institute in Parma (Italy). 
The process pressure is maintained by fixing the flux of sputtering gas (pure 99.999\% Ar) through the fluxmeter and the opening rate of the throttle valve. The substrate, introduced in the sputtering chamber through the load-lock, is screwed to the lateral surface of a cylindrical carousel that can rotate around its axis, coaxial to the cylindrical chamber. As the atoms emitted from the target have a higher concentration along the direction perpendicular to the target surface, the oscillation of the substrate in front of the targets provides films with uniform thickness. The oscillation parameters are selected and controlled by software, allowing the oscillation of the substrate in front of different targets alternatively, which can be used to obtain films with different stoichiometry.

The heater located on the back side of the substrate-holder can reach temperatures up to $600{ }^{\circ} \mathrm{C}$, that are measured by a thermocouple. The real substrate temperature can be calibrated with the heater temperature before the deposition, by means of another thermocouple which can be located at the substrate surface. After heating, the substrate can be cooled down to room temperature with a flux of compressed air on its back side.

The main parameters which need to be optimized when growing films by sputtering are the substrate temperature and the deposition rate of the film material, which depends strongly on the type of sputtering gas and its pressure, the target power, and the sputtering yield, i. e. the number of emitted atoms per incident ion. Therefore, periodical calibrations are necessary in order to obtain the sputtering rates as a function of Ar pressure and target power. 


\subsection{Structural and Morphological Characterization}

The structural and morphological characterization of the films is a fundamental part of this work, as will determine the quality of the films prepared by PLD or sputtering. From this study, different issues such as the crystal structure and lattice parameter, chemical composition of the films, thickness, roughness and the interface structure with the substrate can be extracted. These can be achieved by means of different techniques, including x-ray diffraction (XRD) and reflectivity (XRR), atomic force microscopy (AFM) and transmission electron microscopy (TEM).

All the XRD and XRR measurements shown in this thesis have been performed at the INA in Zaragoza (Spain) except the powder XRD measurements performed for the PLD targets characterization, which were carried out by the $\mathrm{x}$-ray Service at the University of Zaragoza (Spain). The AFM images were taken both at the INA in Zaragoza (Spain) and at the IMEM-CNR research institute in Parma (Italy). Concerning the TEM images, these were obtained by Dr. Magen either at the CEMES - CNRS institute in Toulouse (France), at the Oak Ridge National Laboratory in Tennessee (USA) or at the INA in Zaragoza (Spain).

\subsubsection{X-ray Diffraction}

$\mathrm{X}$-ray diffraction (XRD) is a basic tool for materials science, as it is a nondestructive technique which allows to determine a wide range of properties, ranging from crystallographic structure and chemical composition to physical properties of materials and thin films such as preferred crystalline orientation, lattice parameter (and thus, presence of epitaxial strain), grain size, film thickness and even relative abundance of crystalline compounds when using fitting techniques such as Rietveld refinement.

$\mathrm{XRD}$ techniques are based on the elastic scattering of $\mathrm{x}$-rays from structures with long range order. If an incident monochromatic x-ray beam encounters a crystal lattice, scattering occurs. Although most scattering interferes with itself and is eliminated (destructive interference), diffraction occurs when scattering in a certain direction is in phase with the scattered rays coming from other atomic planes, producing a constructive interference. The condition necessary for constructive interference to occur is given by Bragg's law:

$$
n \lambda=2 d \sin \theta
$$


where $\lambda$ is the wavelength of the characteristic x-rays, $d$ is the lattice interplanar spacing of the crystal, $\theta$ is the Xx-ray incidence angle (Bragg angle, also called $\omega$ ) and $n$ is an integer known as the order of the diffracted beam.

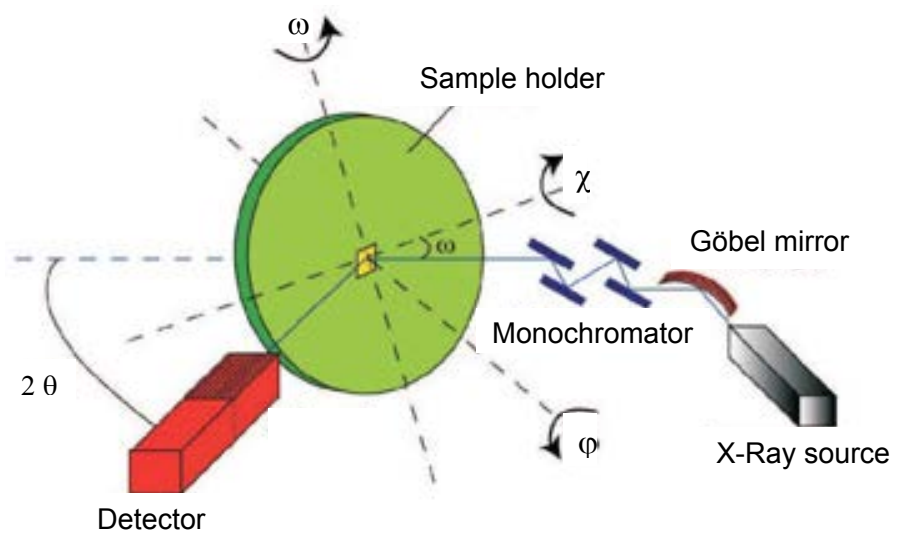

Figure 1.11: Schematic of an x-ray diffractometer [66].

A diffraction pattern is obtained by measuring the intensity of scattered waves as a function of scattering angle, obtaining very strong intensities (Bragg peaks) when scattered waves satisfy the Bragg condition.

The basic geometry of an x-ray diffractometer involves a source of monochromatic radiation and an x-ray detector situated on the circumference of a graduated circle centered on the sample holder (figure 1.11). Divergent slits, located between the x-ray source and the sample, and divergent slits, located between the sample and the detector, limit scattered (non-diffracted) radiation, reduce background noise, and collimate the radiation.

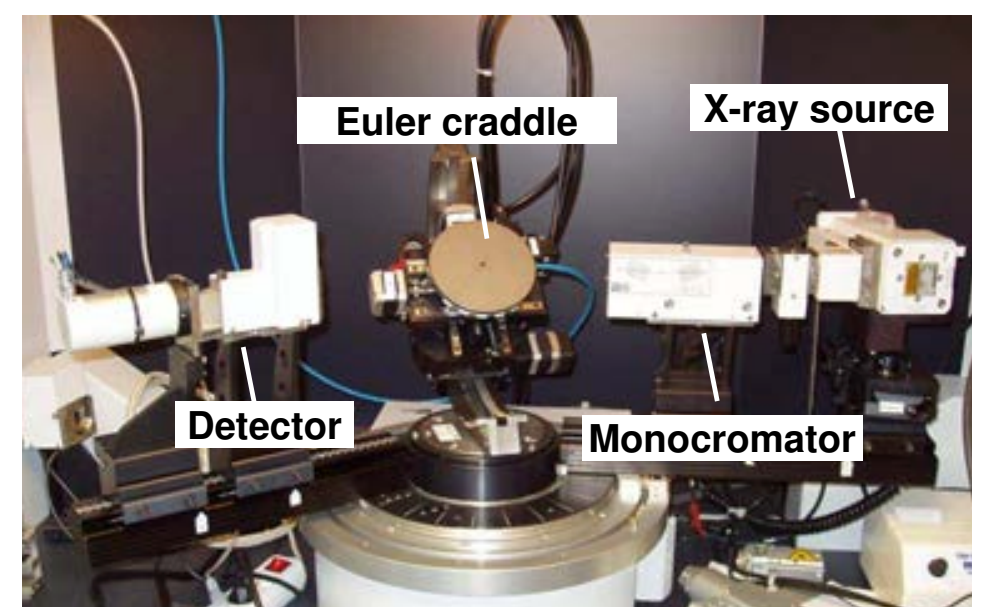

Figure 1.12: HRXRD Bruker D8 Advance equipment at the INA. 
The x-ray diffraction measurements of the films were carried out with the High-Resolution x-ray diffractometer (HRXRD) Bruker D8 Advance equipment, at the INA (figure 1.12). In the used high resolution configuration (HRXRD), the four crystal Ge (220) monochromator selects the $K_{\alpha 1}$ radiation from the $\mathrm{Cu}$ source, providing a x-ray beam with a wavelenght of $\lambda=1.54056$ $\AA$.

With the HRXRD Bruker equipment it is possible to perform different measurements:

- In a typical x-ray diffraction measurement, also called $\theta / 2 \theta$ scan, the detector and sample holder are mechanically coupled with a goniometer so that a rotation of the detector of $2 \theta$ degrees occurs in conjunction a rotation of $\theta$ degrees of the specimen, i.e. a fixed 2:1 ratio. The diffraction peaks position is directly related with the interplanar distance, and gives us information about both the out-of-plane lattice parameter and the crystalline structure, as well as information about the stoichiometry and presence of other phases, as each material with an specific structure has a characteristic pattern. In addition, from the full width at half maximum $\left(\mathrm{FWHM} \equiv \beta_{h k l}\right)$ of a diffraction peak at the position $\theta_{h k l}$, the crystalline grain size $D_{h k l}$ can be extracted by means of the Scherrer formula:

$$
D_{h k l}=\frac{\lambda}{\beta_{h k l} \cos \theta_{h k l}}
$$

- In a rocking curve or $\omega-s c a n$, the intensity from an $h k l$ reflection is measured by keeping $2 \theta$ constant and rotating the sample around an axis perpendicular to the plane defined by the incident and refracted beams. From the rocking curve peak, information about the orientation distribution of the different (hkl) planes (mosaic spread) can be extracted: the lower the FWHM, the better crystallographic orientation has the sample.

- The epitaxy relationship can be demonstrated by means of a $\phi-s c a n$, where an asymmetrical $h k l$ reflection is measured by keeping $2 \theta$ constant and $\omega=\theta$, as a function of the rotation angle around an axis perpendicular to the sample plane $(\phi)$. If the epitaxy relationship is satisfied, all the equivalent reflections from the [hkl] planes should appear.

- Reciprocal Space Maps (RSM) are bidimensional images from a chosen space region that can be obtained by performing several $\theta / 2 \theta$ measurements with an $\omega$ offset between them (figure 1.13). If the reflections measured are asymmetric, both out-of-plane $\left(a_{\perp}\right)$ and in-plane $\left(a_{\|}\right)$lattice parameters can be extracted from the RSM measurement. 
When the RSM region includes reflections from both the substrate and the film, the comparison between their lattice parameters provide information about the epitaxial strain experienced by the film. The diffraction from an asymmetric reflection (hkl) verifies:

$$
\begin{aligned}
\vec{Q} & =\overrightarrow{K^{\prime}}-\vec{K} \\
|\vec{K}| & =\left|\overrightarrow{K^{\prime}}\right|=\frac{2 \pi}{\lambda}
\end{aligned}
$$

Therefore the lattice parameters can be obtained:

$$
a_{\|}=\frac{2 \pi \sqrt{h^{2}+k^{2}+l^{2}}}{Q_{x}} \quad a_{\perp}=\frac{2 \pi \sqrt{h^{2}+k^{2}+l^{2}}}{Q_{z}}
$$

And it can be demonstrated that, if the film is positioned in a "clever direction", the RSM can be performed in such a symmetry that the obtention of the lattice parameters is simpler:

$$
a_{\|}=\frac{2 \pi \sqrt{h^{2}+k^{2}}}{Q_{x}} \quad a_{\perp}=\frac{2 \pi l}{Q_{z}}
$$
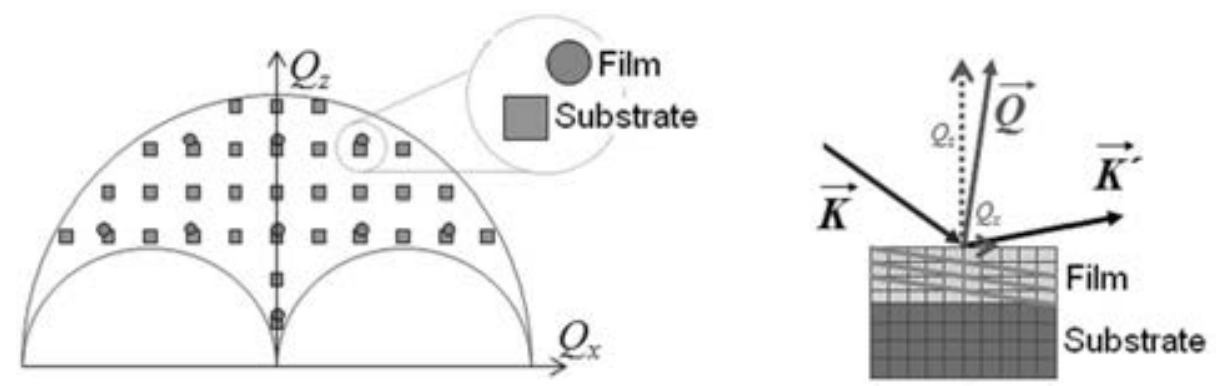

Figure 1.13: Diagram of a full Reciprocal Space Map of a film and its substrate.

\subsubsection{X-ray Reflectivity}

$\mathrm{X}$-ray reflectivity (XRR) is a non destructive characterization technique related to diffraction but which relies on the interference of -rays reflected at interfaces between layers with different refraction index more than crystallographic planes, therefore, it is sensitive to gradients in composition and also in mass density. Film thickness, density and roughness can also be determined with high accuracy by XRR data direct analysis:

In a reflectivity pattern, film thickness (t) can be obtained from the period of interference fringes (Kiessig's fringes) at high angles $(\Delta \alpha)$ (figure 1.14):

$$
t \approx \frac{\lambda}{2 \Delta \alpha}
$$


The critical angle $\left(\alpha_{c}\right)$ depends on the sample density, as shown in figure 1.15 . It can be obtained together with the thickness from the modified Bragg's Law:

$$
\alpha_{n}^{2}=\alpha_{c}^{2}+(n+\Delta n)^{2} \frac{\lambda^{2}}{4 t^{2}}
$$

a $\alpha^{2}$ versus $(n+\Delta n)^{2}$ plot gives both $t$ and $\alpha_{c}$.

Finally, large roughnesses produce a fast decrease in reflectivity intensity. The evolution of oscillation amplitude reveals which interface is rougher: if the amplitude is constant, the roughnesses are similar; if it decreases monotonously, the lighter layer is the rougher; finally, if the amplitude increases and then decreases, it indicates that the denser layer is the rougher.

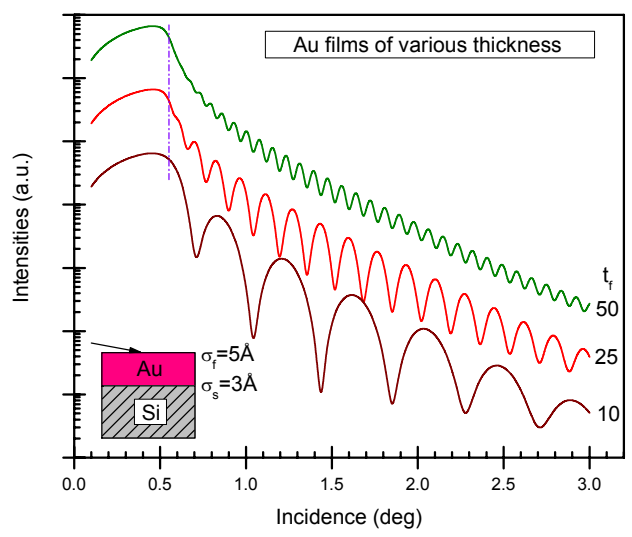

Figure 1.14: Film thickness influence on a XRR pattern.

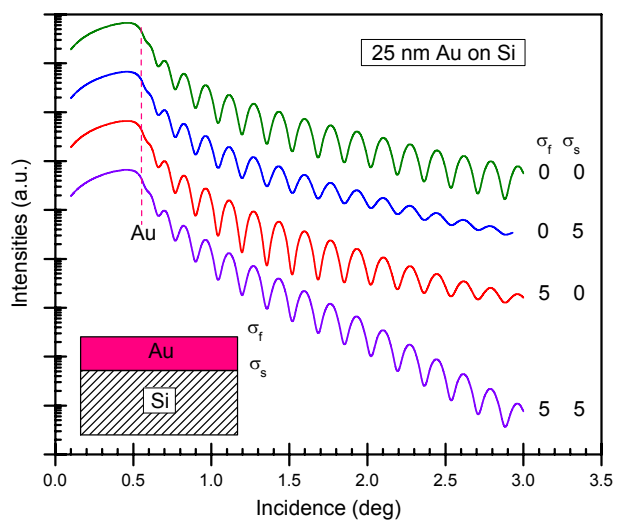

Figure 1.16: Roughness influence on a XRR pattern when the upper layer has a higher density than the substrate.

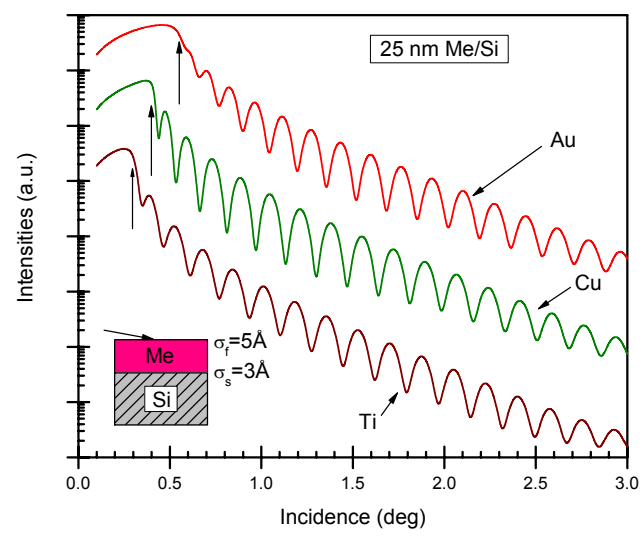

Figure 1.15: Film density influence on a XRR pattern.

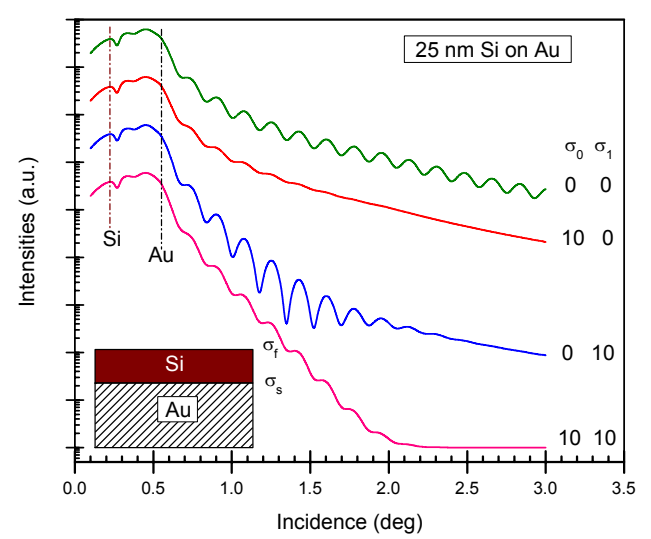

Figure 1.17: Roughness influence on a XRR pattern when the upper layer has a lower density than the substrate [67]. 
The direct analysis works fine for films composed by one homogeneous layer, although it is not useful when analyzing layers with native oxides or multilayers, making a fitting analysis necessary, which would give more accurate results on density, thickness and roughness of all the layers through a proposed model.

The XRR measurements were carried out with the same equipment used of the diffraction measurements: the HRXRD Bruker D8 Advance equipment at the INA (figure 1.12). The roughnesses, thicknesses and densities of the layers and substrates were numerically fitted by means of the commercial software LEPTOS, provided by Bruker Company.

\subsubsection{Atomic Force Microscopy}

Atomic Force Microscopy (AFM) is a type of scanning probe microscope developed by Binning and introduced in 1986 by Binning, Quate and Gerber [68]. This technique take advantage of the relatively strong forces that act between a tip in close proximity to a sample surface, which can range from Van der Waals and capillary forces to electrostatic or magnetic forces.

The tip, with a curvature radius of the order of nanometers, is integrated into a cantilever (usually Si-oxide or Si-nitride, depending on the information we want to obtain from the sample), which is deflected by the sample-tip interaction. This deflection is usually measured by means of a laser beam which is focused on the tip, and the reflected beam is then analyzed by a set of photodiodes.

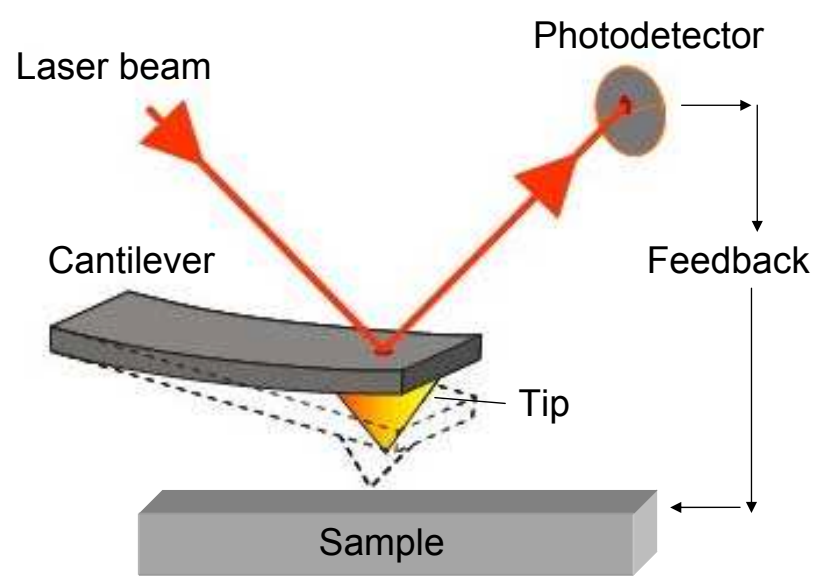

Figure 1.18: Schematic of an AFM setup, adapted from ref. [69].

There are different kind of measurements that can be performed with a AFM: 
- Contact mode, where the tip scans the sample in close contact with the surface.

- Non-contact mode, where the tip hovers 50 - $150 \AA$ above the surface.

- Jumping mode, used to image biomolecules in aqueous media without establishing any mechanical contact between the tip and the sample.

- Tapping mode. In this scanning mode, the cantilever is oscillated close to its fundamental frequency. The oscillation amplitude, phase and frequency are modified by the tip-sample surface interaction forces, providing information about the surface characteristics. Tapping mode can be operated either in frequency modulation operation or amplitude modulation, being the second the most common.

Tapping mode is the technique usually employed in AFM microscopy when studying surface topography and roughness, as it allows high resolution topographic imaging of sample surfaces that are easily damaged, loosely hold to their substrate, or difficult to image by other AFM techniques.

All the AFM images obtained in this thesis, have been obtained in tapping mode. While the AFM images shown in chapter 2 were obtained in a Nanotech AFM microscope in the INA at Zaragoza (Spain), the AFM images in chapters 3 and 4 were taken at the IMEM-CNR research institute at Parma (Italy), with a multimode scanning probe microscope by Veeco, equipped with a Nanoscope IIIa controller.

\subsubsection{Transmission Electron Microscopy}

The Transmission Electron Microscopy (TEM) prototipe was built by Max Knoll and Ernst Ruska in 1931, and eight years later the first commercial microscope was available.

In this microscopy technique, a beam of electrons formed by an electron source is accelerated by means of a positive electrical potential and transmitted through an ultra thin specimen, interacting with the specimen as it passes through and forming the image. The resulting image is magnified and focused onto an imaging device, such as a fluorescent screen, on a layer of photographic film, or to be detected by a sensor such as a CCD camera. The specimen needs to be ultra thin in order to allow the incoming electron beam cross through it, and therefore, the sample is cut and polished until its thickness is only some hundred nm. 
Morphology information about the sample can be extracted with a TEM, and also crystallographic properties like lattice parameter or atomic-scale defects when working in high resolution, as this microscope has enough resolution to "see" the atoms. If the TEM is equipped with an Electron Energy Loss Spectrometer (EELS) for the outcoming electrons, their energy loss can be measured, and as this loss is due to scattering effects specific for the different elements of the sample, the composition of the studied image can be deduced. A TEM equipment can be also improved by adding optical components in order to correct spherical aberrations.

All the TEM images shown in this thesis have been obtained by C. Magen in different equipments. The magnetite images in Chapter 2 were taken in a Philips $C M 30 \mathrm{LaB}_{6}$ microscope at the CEMES - CNRS institute in Toulouse (France). The images in Chapter 3 were obtained in the VG HB603U and HB501UX STEM Microscopes equipped with a Nion aberration corrector at the Oak Ridge National Laboratory in Tennessee (USA) or at the Titan TEM at the INA in Zaragoza (Spain).

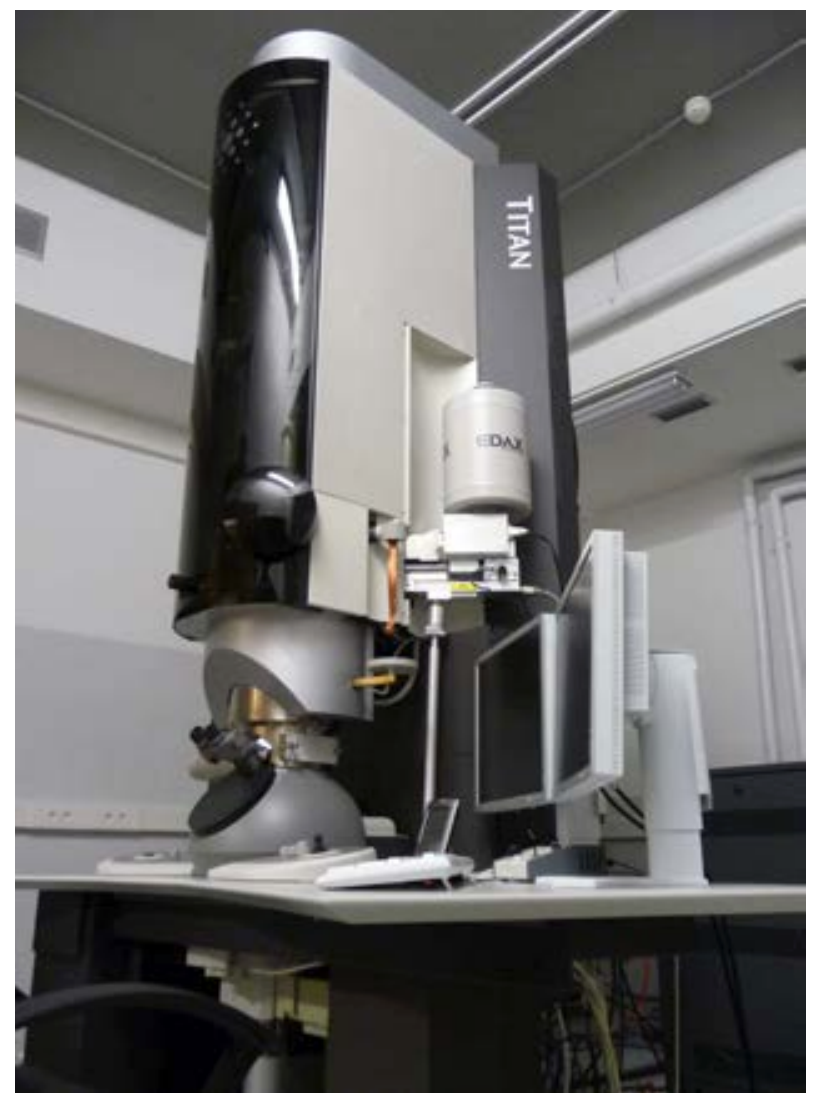

Figure 1.19: Image of the Titan TEM at the INA in Zaragoza (Spain). 


\subsection{Magnetic Characterization}

The magnetic properties of the samples have been mainly studied by two techniques: Superconducting Quantum Interference Device (SQUID) and Vibrating Sample Magnetometry (VSM). I will also introduce other techniques such as Alternating Gradient Force Magnetometry (AGFM) and Magnetic Force Microscopy (MFM) that have been used to characterized the FePt samples described in chapter 4, and Polarized Neutron Reflectivity (PNR) and X-ray Magnetic Circular Dichroism spectroscopy (XMCD) experiments, performed in large installations as part of the ultra-thin magnetite study in chapter 2.

\subsubsection{SQUID magnetometry}

Superconducting Quantum Interference Device magnetometry is one of the most effective and sensitive ways of measuring magnetic properties. It consists of two superconductors separated by thin insulating layers to form two parallel Josephson junctions, and therefore, SQUID magnetometers require cooling with liquid helium $(4.2 \mathrm{~K})$ or liquid nitrogen $(77 \mathrm{~K})$ to operate.

Following the equations established by Brian David Josephson in 1962, the electrical current density through a weak electric contact between two superconductors depends on the phase difference of the two superconducting wave functions. Moreover, in a superconducting ring with one (so-called rf SQUID) or two (dc SQUID) weak contacts, the phase time derivative is influenced by the magnetic flux through this ring. Therefore, such a structure can be used to convert magnetic flux obtained by moving the sample up and down into an electrical voltage.

By locking the frequency of the readout to the frequency of the movement (RSO, reciprocating sample oscillation), the magnetometer system can achieve extremely high sensitivity for ultra small magnetic signals such as $1 \cdot 10^{-8} \mathrm{emu}$.

The magnetometers used in this thesis are MPMS-XL and MPMS-5S DCSQUID models from Quantum Design, and are located at the Magnetic Measurements Service of the University of Zaragoza (Spain). The maximum applied magnetic field is $5 \mathrm{~T}$, and the temperature can be decreased down to 2 $\mathrm{K}$ (Helium-cooled), and the sensitivity can be increased by means of the RSO option in the MPMS-XL equipment. 


\subsubsection{Vibrating Sample Magnetometry}

The VSM is one of the most utilized techniques to measure basic magnetic properties as a function of the applied magnetic field in a wide range of temperatures, with a sensitivity of $\sim 10^{-6} \mathrm{emu}$. On of the main advantages of this technique is the sample positioning system, which facilitates the orientation of the samples and also allows to measure relatively big samples. In addition, VSM has a lower cost than other magnetometry techniques, as the magnetic field is provided by a water-cooled electromagnet, avoiding the use of liquid He or $\mathrm{N}_{2}$, which is required in SQUID magnetometry.

The sample is placed in a constant magnetic field created by an electromagnet and moved up and down at a frequency of $75 \mathrm{~Hz}$ perpendicularly to the magnetic field direction. As the sample is moved, the magnetization changes as a function of time producing an induced current in a set of pick-up coils according to Faraday's Law of induction, which is and lock-in detected in the VSM detection module. When the sample moves in a direction, the magnetic flux increases in one of the coils while decreases for the other cancelating the variations due to the homogeneous field. Therefore, only the changes due to the magnetization from the sample are registered.

The VSM equipment used in this thesis is a model from ADE Technologies (now MicroSense LLC), located at the INA at the University of Zaragoza (Spain) (Figure 1.20).

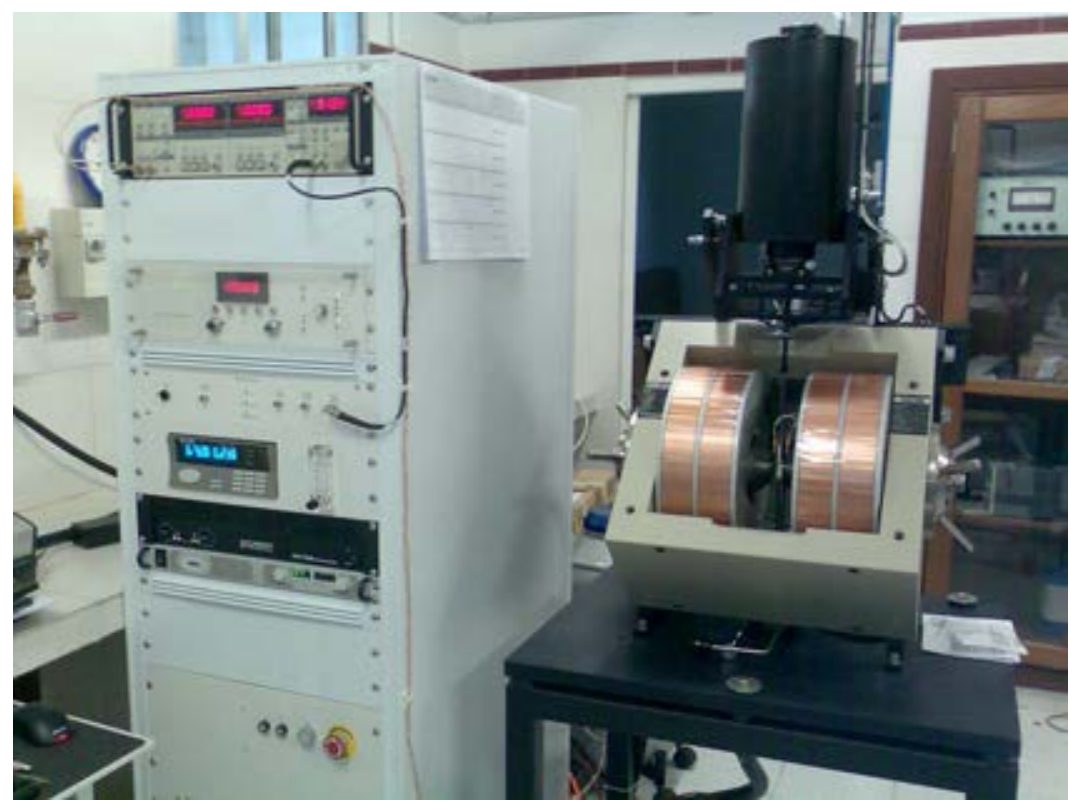

Figure 1.20: VSM equipment from ADE Technologies at the INA (Zaragoza, Spain). 
The maximum applied magnetic field is $1.8 \mathrm{~T}$, although it can be increased up to $2 \mathrm{~T}$ by decreasing the distance between the electromagnet pole pieces, and the measurements as a function of the temperature can be performed from $60 \mathrm{~K}$ to $800 \mathrm{~K}$. The system has sample holders designed for the measurement of powder and films of different sizes up to areas of $1.2 \mathrm{~cm} \times 1.2 \mathrm{~cm}$.

\subsubsection{Magnetic Force Microscopy}

MFM is a technique derived from the AFM that images the spatial variation of magnetic forces on a sample surface by means of a tip coated with a ferromagnetic film. The system operates in non-contact mode, revealing the variation in oscillation frequency or phase induced by the magnetic field's dependence on tip-to-sample separation when the tip is forced to oscillate above the sample surface. The topographic profile of each scan must be measured in first place in order to track the probe tip at a constant height above the sample surface during the second pass, extracting the magnetic signal.

This is a powerful technique for the research in sub-micron magnetization patterns and structures, since a lateral resolution below $50 \mathrm{~nm}$ can be reached. The field dependence of domain structures and magnetic reversal process can also be observed by applying an external field during the measurement. However, although little sample preparation is required, the images are difficult to quantify. This means that the use of an MFM should be completed with other magnetometry techniques such as Magneto Optic Kerr Effect (MOKE), VSM, AGFM or SQUID.

\subsubsection{Alternating Gradient Force Magnetometry}

AGFM technique is based is on the application of an alternated field-gradient, in order to produce a periodic force on a sample set in a uniform field [70]. The sample is mounted on a quartz probe that transmits the deflection due to the field-gradient to a piezoelectric sensor, producing a voltage proportional to the force, and hence to the magnetic moment of the sample. The sample can be oriented either parallel or perpendicular to the applied magnetic field by using two different probes, allowing the accurate measurement of magnetic out-of-plane signal of the films.

It is a high sensitivity technique $\left(10^{-8} \mathrm{emu}\right)$ for the measurement of magnetic moments as a function of an applied magnetic field, as the piezoelectric signal is processed by a lock-in system, thus eliminating most of the noise; and moreover, the measurements are done at the mechanical resonance of the probe $(\approx 500 \mathrm{~Hz})$, with the consequent amplification of the signal. 
The magnetometer employed during this thesis is a Micromag 2900 from Princeton Measurements Corporation. It is located at the IMEM-CNR research institute in Parma (Italy), and operates at room temperature with a maximum magnetic field of $20 \mathrm{kOe}$.

\subsubsection{Polarized Neutron Reflectometry}

PNR is a specially suited technique to the measurement of magnetization and magnetic depth profile in thin films and heterostructures.

In this technique, a highly collimated beam of neutrons is directed onto the sample, and the reflected radiation is measured either as a function of the angle or the neutron wavelength. As neutrons can be reflected from surfaces in the same way as X-rays and electrons, all the formalisms developed for XRR are valid for neutron reflectivity. Moreover, due to the neutron spin, the structure and magnetic order can be obtained simultaneously from a PNR experiment. Therefore, detailed information about the structure of the sample, including thickness, density and roughness of all the sample layers, is also provided by the shape of the reflectivity profile (see Section 1.2.2 in this chapter).

For a magnetic system, an equivalent neutron "optical index" can be derived from the Schrödinger equation. If absorption is neglected, the value of the optical index is given by:

$$
n^{ \pm} \approx 1-\delta_{N} \pm \delta_{M}=1-\frac{\lambda^{2}}{2 \pi} \rho \pm \frac{m \lambda^{2}}{h^{2}} \mu \cdot B
$$

were $\delta_{N}$ is the nuclear contribution to the optical index, and $\delta_{M}$ is the magnetic contribution, whose sign depends on the relative orientation of the neutron spin with respect to the magnetization (parallel or antiparallel). Thus, both optical indexes $n^{+}$and $n^{-}$can be measured by using polarized neutrons, information about the amplitude and direction of the magnetization being obtained.

It should be noticed that the magnetic optical index is of the same order of magnitude as the nuclear optical index, thus, the strength of the magnetic scattering signal is very similar to that of the nuclear scattering signal. However, neutrons are only sensitive to the magnetic induction and thus cannot distinguish between spin and orbital moment [71].

Disadvantages of neutron reflectometry include the necessity of a neutron source and the fact that some materials may become radioactive upon exposure to the beam. Moreover, this technique has low flux and high background, being necessary long measurement times and a careful treatment of the obtained data. 
The magnetic moment and roughness are obtained together with thickness, density and structural roughness by means of numerical fits, which can be performed by means of different software tools. In our case, the PNR data have been optimized and the background signal subtracted by means of LAMP software, whereas data analysis has been performed either with Parrat or GenX software [72], with film thickness, roughness, and magnetic moment, and substrate roughness used as variable parameters.

The PNR data studied in section 2.4, chapter 2 have been obtained in the D17 reflectometer at the Institute Laue-Langevin (ILL) in Grenoble (France). Similar results were obtained from an analogous experiment performed in the Polref instrument at the ISIS pulsed neutron and muon source at the Rutherford Appleton Laboratory in Oxfordshire (United Kingdom).

The reflectometer D17 operates in three modes, time-of-flight, monochromatic and polarising. The time-of-flight mode is performed by a double chopper system with variable phase and separation, having a useful wavelength range of 2-20 $\AA$; the upper limit set by a frame overlap mirror. The other two modes use multilayer monochromators followed by a composite Ni mirror device to remove long wavelength contamination, running at a fixed wavelength of $5 \AA$ with a base resolution of $4 \%$ [73]. The measurements carried out in this thesis were performed in the monochromatic mode.

The instrument is suitable for the analysis of surface structures, buried interfaces and in-plane correlations in solids and solid/liquid interfaces, and the wide angle multidetector allows the simultaneous measurement of background and off-specular scattering. 


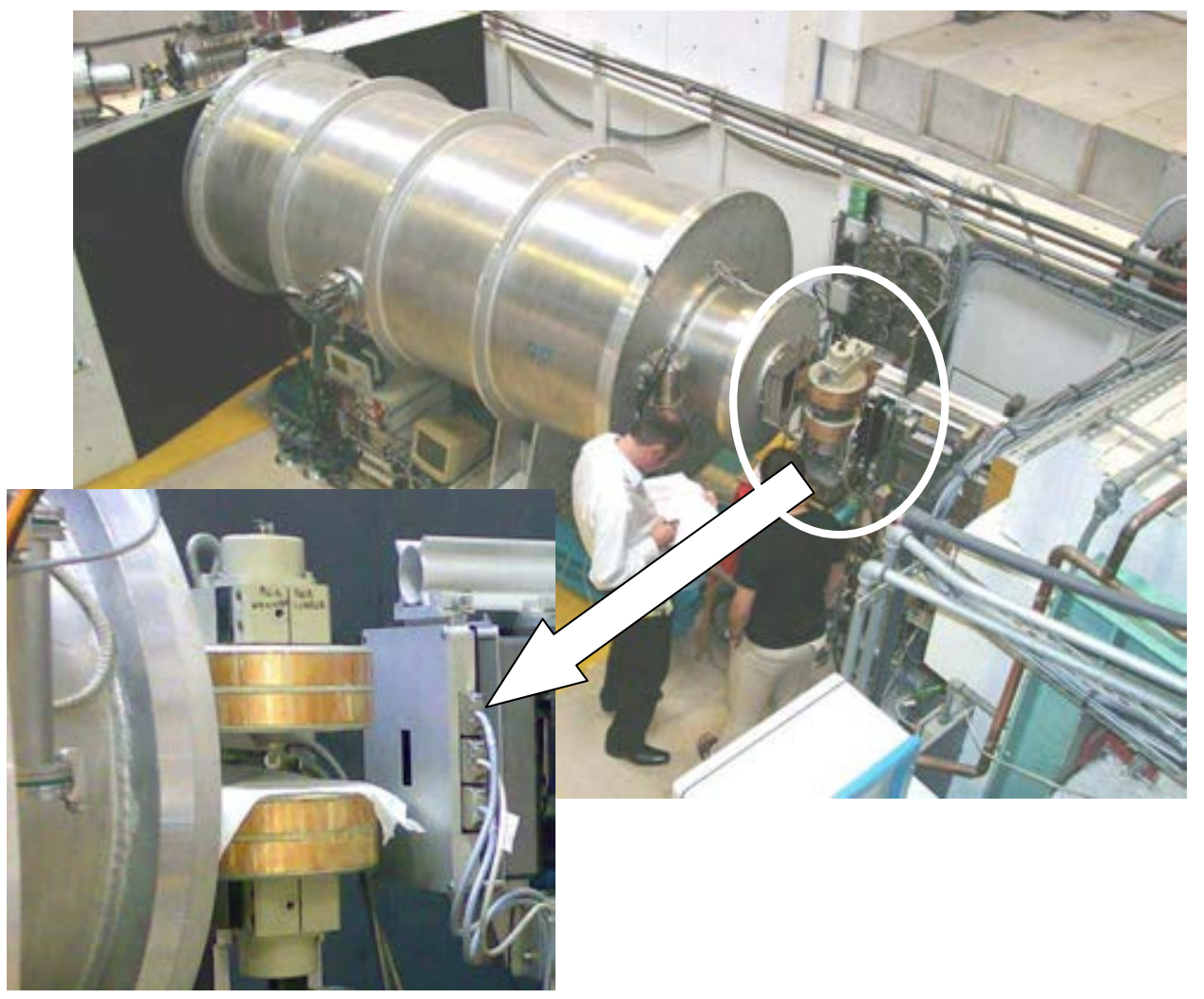

Figure 1.21: Image of the D17 reflectometer at the Institute Laue-Langevin (ILL) in Grenoble (France) 


\subsection{Magnetotransport}

Most of the electrical transport properties as a function of the temperature and magnetic field of the samples studied in this thesis, have been measured at the ICMA in Zaragoza (Spain).

The equipment consists on different elements (Figure 1.22):

- Keithley ${ }^{\circledR} 6220$ DC current source.

- Keithley ${ }^{\circledR} 2182 \mathrm{~A}$ nanovoltmeter.

- A Cryocon ${ }^{\circledR}$ Closed-Cicle-Refrigerator (CCR), which, by means of a thermodynamic cycle in He gas can reach temperatures as low as $25 \mathrm{~K}$. A temperature controller allows the temperature stabilization and the performance of temperature ramps.

- A Walker Scientific electromagnet that can reach a magnetic field of 11 $\mathrm{kOe}$. The magnetic field is measured by means of a Hall Sensor located between the electromagnet coils.
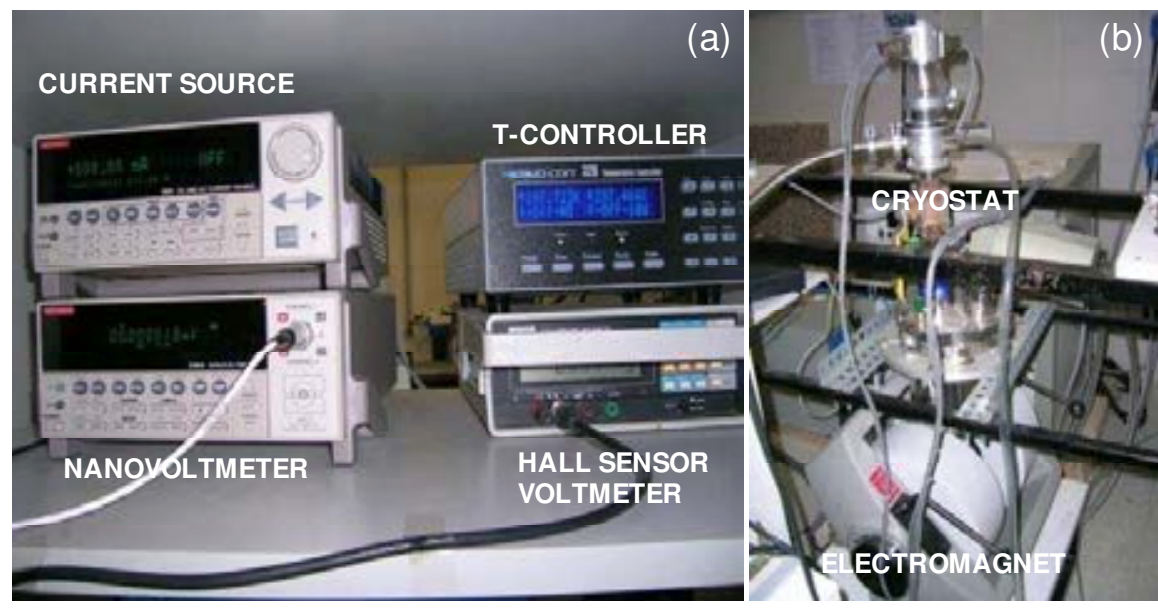

Figure 1.22: Magnetotransport measurements equipment at the ICMA. (a) Electrical measurement equipments, temperature controller and magnetic field visualizer. (b) View of the CCR and the electromagnet.

The system can be PC-controlled by means of Labview programs, developed by Dr. Amalio Fdez-Pacheco and Dr. Jan Michalik from our research group.

The transport measurements performed on magnetite films in chapter 2 have been performed in a 4-probe configuration in patterned samples by optical lithography in order to avoid contact resistances and voltage offsets in the measurements. 
Concerning the magnetotransport measurements in high static magnetic field detailed in chapter 2, they were performed at the High Field Magnet Laboratory (HFML) of the University of Nijmegen (Netherlands), where magnetic fields as high as $33 \mathrm{~T}$ can be generated. The experiment was performed with the assistance of our local contacts Dr. Uli Zeitler and Erik Kampert.

All the other magnetotransport measurements were performed by means of the well known Van der Pauw technique, including the transport properties of $\mathrm{SrTiO}_{3} / / \mathrm{Sr}_{2} \mathrm{CrReO}_{6}$ detailed in chapter 3, which were carried out in a commercial Physical Properties Measurement System (PPMS) from Quantum Design at the Instrumentation Service of the University of Zaragoza (Spain).

Van der Pauw technique [74] provides the resistance value of a film sample independently of its shape. If we take a flat lamella (completely free of holes), perform four very small contacts at arbitrary places on the periphery $(\mathrm{M}, \mathrm{N}, \mathrm{O}$ and $\mathrm{P}$ ), we apply a current $I_{M N}$ and measure the potential difference $V_{P}-V_{O}$, and analogously, we apply a current $I_{N O}$ and measure $V_{M}-V_{P}$, it can be demonstrated that:

$$
\exp \left(-\frac{\pi d}{\rho} R_{M N, O P}\right)+\exp \left(-\frac{\pi d}{\rho} R_{N O, P M}\right)=1
$$

where $d$ is the thickness of the lamella, $\rho$ the resistivity of the material, and:

$$
R_{M N, O P}=\frac{V_{P}-V_{O}}{I_{M N}} \quad R_{N O, P M}=\frac{V_{M}-V_{P}}{I_{N O}}
$$

When the sample possesses a line of symmetry the situation is easier, as if $\mathrm{M}$ and $\mathrm{O}$ are placed on the line of symmetry and $\mathrm{N}$ and $\mathrm{P}$ are symmetrical with respect to this line (Figure 1.23), then $R_{N O, P M}=R_{M N, O P}$. And taking into account that $R_{P M, N O}=R_{M N, O P}$, from equation 1.10 it follows that:

$$
\rho=\frac{\pi d}{\ln 2} R_{M N, O P}
$$

Therefore, one measurement is enough to obtain the desired resistivity.

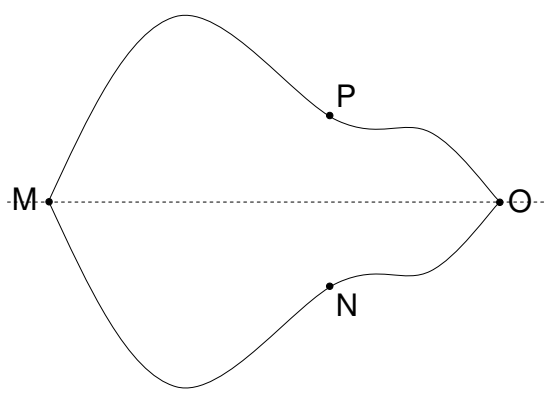

Figure 1.23:

Situation were the resistivity measurement is simplified due to the symmetry of the sample. 


\subsection{Lithography}

Lithography, from the Greek lithos, "stone", and grapho, "to write", is a method for printing widely used from ancient times. Nowadays, the lithography process has acquired renewed interest due to its application to micro- and nanotechnology, and leading to new micro-lithography and nano-lithography techniques, which allow material structuring on a fine scale.

In a lithography process, a previously designed pattern is transferred to a sample by means of different techniques, the most common being:

Photolithography: It is the most commercially advanced form of microlithography, and generally uses previously designed and fabricated photomasks as a master to derive the final pattern.

Electron-beam lithography: The final pattern is created by scanning a resist-coated substrate with an electron beam controlled with a computer, following the desired design. Although electron-beam lithography can reach higher patterning resolution than photolithography (few nm) and no mask is required, it is a much slower technique.

In order to define the proper geometry for magnetite transport measurements in Chapter 2, photolithography processes were carried out by Dr. Amalio Fernández Pacheco, Dr. Javier Sesé together with the technical staff of the Lithography Laboratory (R. Valero, I. Rivas, and R. Cordoba) at the 10,000 class-clean room available at the INA in Zaragoza (Spain). The main process stages are schematized in figure 1.24

Preliminary TMR measurements (presented in Chapter 5) have been obtained from Magnetic Tunnel Junctions (MTJs) processed in the class 100 and class 10 areas at the INESC Microsystems and Nanotechnologies Research Institute in Lisbon (Portugal), under the supervision of Susana Pinheiro Cardoso de Freitas.

\subsubsection{Photolithography standard procedure}

Depending on the pattern that is to be defined, the total photolithographic process will combine several steps in sequence, including:

Cleaning: The organic or inorganic contaminations that may be present on the wafer surface are usually removed by a chemical treatment which depends on the material wafer and an annealing to a temperature high enough to dry and remove any moisture at the wafer surface. 
Wafer preparation: Prior to the photoresist deposition, an "adhesion promoter" needs to be deposited on the wafer surface. This composites are water repellent, which prevents the aqueous developer from penetrating between the photoresist and the wafer surface, and would produce the lifting of photoresist portions in the developing step.

Photoresist application: The wafer is covered with a uniform thin photoresist (either positive or negative) layer by spin coating. Usually the photo resist-coated wafer is then soft-baked in order to remove the solvents from the resist, minimize the stress and improve the adhesion.

Exposure: The wafer coated with the photoresist is exposed to a pattern of intense light (usually ultraviolet light) by means of a photomask or directly with a laser beam. While the positive photoresist becomes soluble in a given developer, the negative photoresist becomes insoluble.

Developing: A photographic developer is used to remove the developer-soluble parts of the photoresist. By means of a baking the remaining photoresist is solidified, producing a more durable protecting layer on the wafer.

Etching: In this step, the upper layers of the wafer that are not protected with the photoresist are removed either by a liquid (wet etching) or a plasma (dry etching).

Photoresist removal: When the photoresist is no longer needed, it is removed by a liquid "resist stripper".

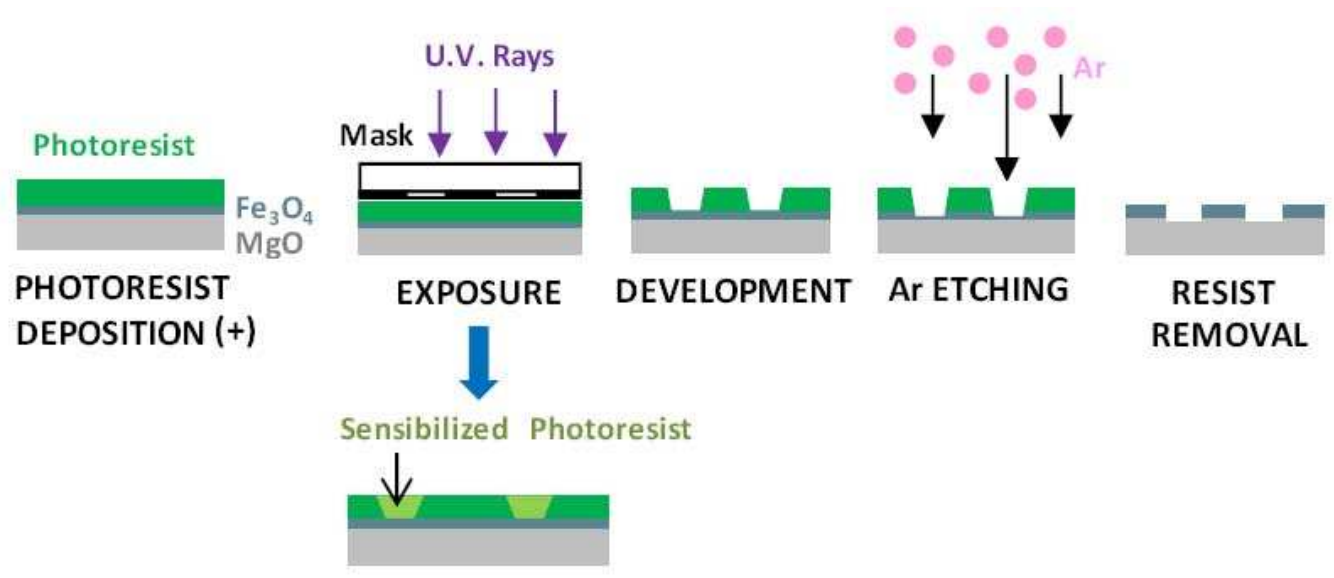

Figure 1.24: Photolithography procedure performed on the $\mathrm{Fe}_{3} \mathrm{O}_{4}$ thin films in order to obtain the optimal geometry for transport measurements (the baking steps are omitted) [75]. 
The photolithography procedure necessary for the obtention of MTJs requires additional preparation stages, depending on the heterostructure to be lithographied. The specific complete process performed in this thesis is detailed in chapter 5, section 5.4, 


\section{Chapter 2}

\section{Magnetite epitaxial thin films}

\subsection{Magnetite: an overview}

There are four different iron oxides abundantly found in nature: wüstite $(\mathrm{FeO})$, magnetite $\left(\mathrm{Fe}_{3} \mathrm{O}_{4}\right)$, maghemite $\left(\gamma-\mathrm{Fe}_{2} \mathrm{O}_{3}\right)$ and hematite $\left(\alpha-\mathrm{Fe}_{2} \mathrm{O}_{3}\right)$ (see figure 2.1), which have been widely known and used in ancient times, specially the hematite and the magnetite, due to their magnetic properties. More specifically, $\mathrm{Fe}_{3} \mathrm{O}_{4}$ is found in nature in rocks called lodestone, and it is thought that its name "magnetite" is derived from the ancient locality Magnesia, near Macedonia, where magnetite was first discovered around 2000 BC. It was the first cubic crystal shown to be magnetically anisotropic [76].

$\mathrm{Fe}_{3} \mathrm{O}_{4}$ is a half-metallic ferrimagnet actively investigated due to its high Curie temperature $(860 \mathrm{~K})$ [77, 78], and its characteristic metal-insulator firstorder transition at $120 \mathrm{~K}$ (Verwey transition) [79 81]. Not only is it magnetic at room temperature, but also theoretical calculations reveal a half-metallic behavior at room temperature with $100 \%$ negative spin polarization at the Fermi level [82 84], calculations which were later supported by x-ray magnetic circular dichroism [85], MOKE [84], photoemission spectroscopy [78, 86], tunneling magnetoresistance [87], and spin polarized scanning tunneling spectroscopy measurements [88], evidencing indeed a high negative spin polarization of $\mathrm{Fe}_{3} \mathrm{O}_{4}$. Therefore, this material is particularly interesting to integrate into spintronic devices such as MTJs [89] because of its potentiality to exhibit large tunnel magnetoresistance. 
However, despite the extensive research, some of the properties of $\mathrm{Fe}_{3} \mathrm{O}_{4}$ are still not understood, specially the nature of the Verwey transition [86, 90, 91]. Therefore, in recent years both magnetite single crystals and thin films have been synthesized and further theoretical and experimental work has been performed.

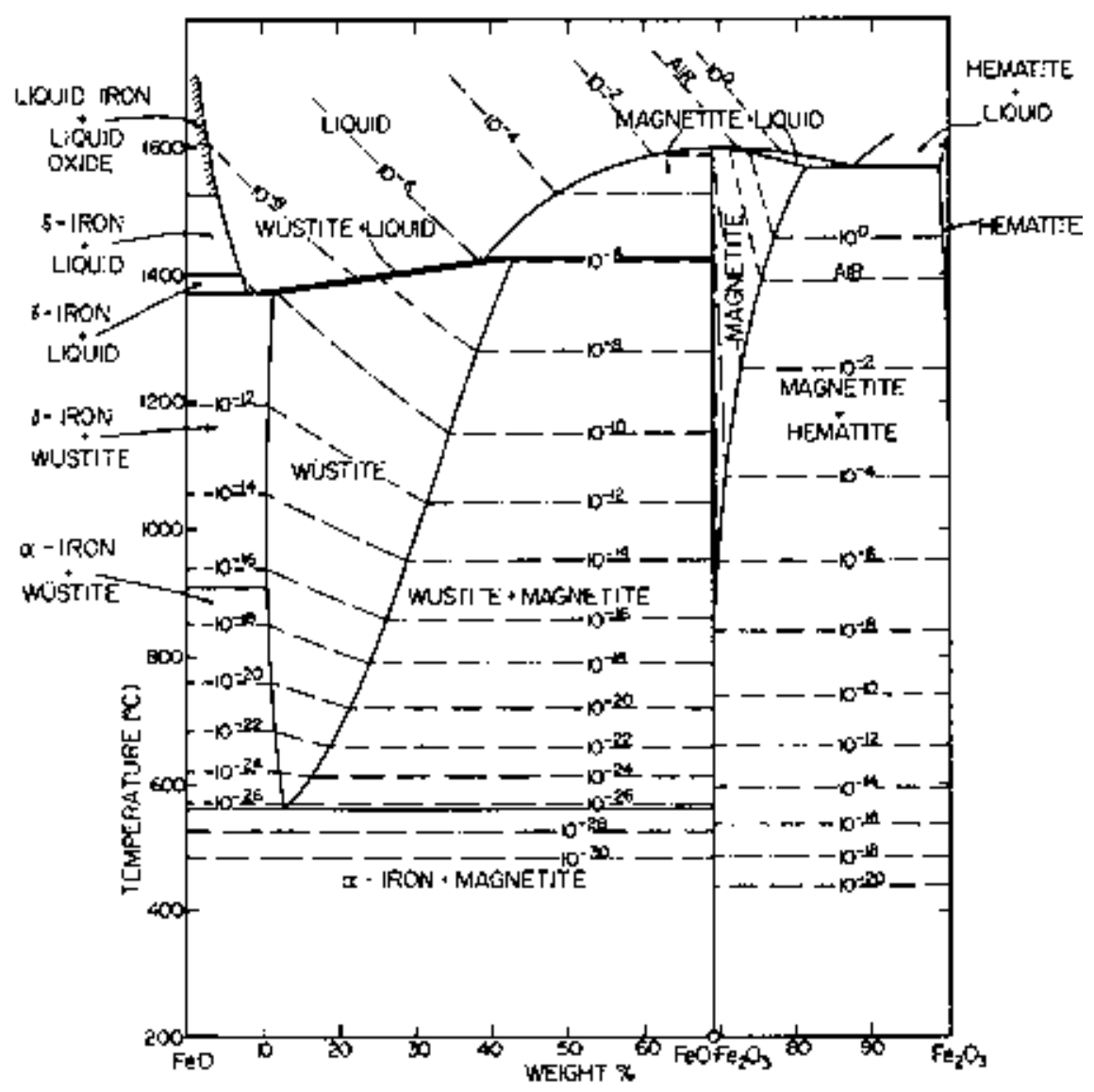

Figure 2.1: Phase relations of the system $\mathrm{FeO}-\mathrm{Fe}_{2} \mathrm{O}_{3}$, with solid lines marking boundaries between respective phases and dash-dotted curves indicating oxygen isobars, indexed in atm units 92].

\subsubsection{Structure and properties of bulk $\mathrm{Fe}_{3} \mathrm{O}_{4}$}

The chemical name ferrous-ferric oxide indicates that magnetite has a ferrous $\mathrm{FeO}$ (wüstite) and a ferric component $\mathrm{Fe}_{2} \mathrm{O}_{3}$ (hematite), with 8 ferrous and 16 ferric Fe atoms respectively. Magnetite is the simplest of the ferrites family and crystallize in the inverse spinel group [93], being the lattice parameter $a$ $=8.3967 \AA$. 
In the inverse spinel structure, specified generally as $\mathrm{AB}_{2} \mathrm{O}_{4}$, two usually non equivalent metal ions, $\mathrm{A}$ and $\mathrm{B}$, are embedded in a cubically face-centered lattice of $\mathrm{O}^{2-}$ ions. The structure is cubic, with space group $\mathrm{Fd} \overline{3} \mathrm{~m}$, and from the ionic point of view, the magnetite general chemical formula can be written as $\left[\mathrm{Fe}^{3+}\right]_{A}\left[\mathrm{Fe}^{2+}, \mathrm{Fe}^{3+}\right]_{B} \mathrm{O}_{4}$, where octahedral iron ions are indicated as red circles (B sites) and the tetrahedral iron ions as blue circles (A sites) (see figure 2.2). Therefore, $\mathrm{Fe}^{2+}$ and $\mathrm{Fe}^{3+}$ ions coexist at the same crystallographic site in the inverse spinel structure.

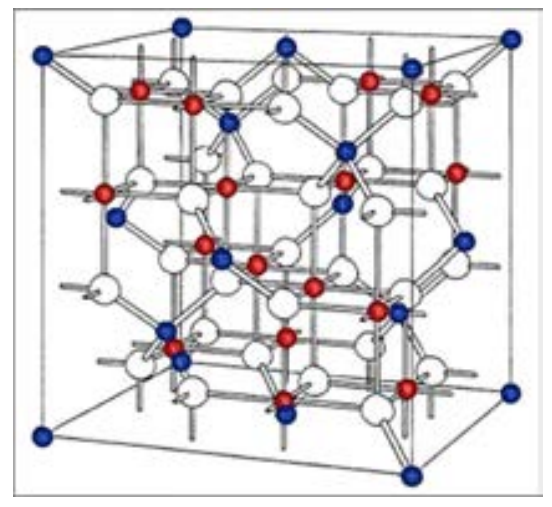

Figure 2.2: The crystal structure of spinel $\mathrm{Fe}_{3} \mathrm{O}_{4}$. Blue atoms are tetrahedrally coordinated $\mathrm{Fe}^{2+}$; red atoms are octahedrally coordinated, 50/50 $\mathrm{Fe}^{2+} / \mathrm{Fe}^{3+}$; white atoms are oxygen [94].

Below $\mathrm{T}_{C}=860 \mathrm{~K}$ magnetite orders ferrimagnetically [95]. This magnetic ordering follows Néels two-sublattice model and implies that the A and B site ions are aligned ferromagnetically within each sublattice, and antiferromagnetically between the two sublattices. The saturation magnetic moment has been determined to be $4.1 \mu_{B} / f . u$. at $300 \mathrm{~K}$, which supports the validity of this model, which predicts:

$$
\begin{gathered}
\mu_{\text {TOTAL }}=\mu\left(F e^{3+} B\right)+\mu\left(F e^{2+} B\right)-\mu\left(F e^{3+} A\right)=5 \mu_{B}+4 \mu_{B}-5 \mu_{B} \\
\mu_{T O T A L}=4 \mu_{B}
\end{gathered}
$$

\section{The Verwey transition}

Around $120 \mathrm{~K}$ magnetite undergoes a phase transition nowadays referred to as the Verwey transition. This transition has been widely studied during the last century. It started in 1929 when Russell W. Millar found a maximum in the specific heat of magnetite at $114.15 \mathrm{~K}$ [96], three years later C. H. $\mathrm{Li}$ reported that the magnetic properties of the crystal abruptly changed at the same temperature [97] and the following years, other authors studied the change in magnetite physical properties at this first-order transition [76]. 
However, it was E.J.W. Verwey who finally gave name to the new transition (Verwey transition hereafter) when characterized it as an order-disorder transition and proposed an ionic model to explain the strong change in the electrical transport properties [79, 80]. This was the first report on a charge ordering phenomenon. According to his model, above the Verwey transition temperature $\left(\mathrm{T}_{V}\right), \mathrm{Fe}^{3+}$ and $\mathrm{Fe}^{2+}$ ions are dynamically disordered in the lattice and electron hopping between them occurs at the B sites. It results in a high conductivity since $\mathrm{Fe}^{3+}$ has $5 \mathrm{~d}$ electrons which are parallel to each other and form a filled subshell, whereas $\mathrm{Fe}^{2+}$ has an additional spin-down electron which can easily hop to a neighboring $\mathrm{Fe}^{3+}$ site if their spins are parallel. At the Verwey transition the structure distorts from cubic symmetry [81] and a charge ordering presumably occurs at the $\mathrm{B}$ sites, a long range spatial ordering of the $\mathrm{Fe}^{3+}$ and $\mathrm{Fe}^{2+}$ ions which localizes the electron preventing the motion of carriers and reducing the conductivity by two orders of magnitude.

Usually, above the Verwey transition the electronic conduction is described by a thermally activated hopping process between the ferrous and ferric ions in the octahedral lattice sites, and the random distribution of the ions leads to an isotropic conductivity. Below the transition the conductivity has a rather complex character [98]. Pai and Honig [99] showed that in high purity single crystals, the behavior between about $0.5 \mathrm{~T}_{V}$ and $\mathrm{T}_{V}$ is activated type (equation 2.1), while below $0.5 \mathrm{~T}_{V}$ it exhibits a variable range hopping mechanism (equation 2.2), reported previously by Drabble and co-workers [100].

Thermally-activated hopping transport:

$$
\rho=\rho_{0} e^{E_{a} / k T}
$$

Mott's formula for variable range-hopping:

$$
\rho=\rho_{0} e^{\left(E_{a} / k T\right)^{1 / 4}}
$$

Where $\mathrm{E}_{a}$ is the activation energy, $\mathrm{k}$ is the Boltzman constant, $\mathrm{T}$ is the temperature and $\rho_{0}$ is the resistivity constant. The activation energy is the energy required for the charge exchange between ferrous and ferric ions at the octahedral site, and for a magnetite bulk single crystal is $\sim 60 \mathrm{meV}$ at $\mathrm{T}>$ $\mathrm{T}_{V}$, and $\sim 105 \mathrm{meV}$ when $\mathrm{T}<\mathrm{T}_{V}[101]$.

The symmetry for the low-temperature phase of magnetite was proposed by Verwey to be tetragonal [81]. However, following authors considered a rhombohedral geometry [102, 103], and even an orthorhombic phase [104], until it was finally identified as monoclinic [105] by means of neutron diffraction experiments. 
Concerning the transition nature, it was identified by Brabers et al. [106] as not primarily induced by magnetic interactions, the ordering mechanism being related to the second-neighbor Coulomb interaction of the cations on the octahedral sites. Nevertheless, no conclusive structural model or charge ordered arrangement has been identified yet because of the complexity of the low temperature structure.

In nominally stoichiometric materials the transition temperatures have been reported in the 119 - $124 \mathrm{~K}$ range. External applied pressure and the presence of vacancies and impurities are some of the factors which can produce a change in $\mathrm{T}_{V}$, providing an accurate method to probe the quality of magnetite crystals. Elevated applied pressures induce a decreased $\mathrm{T}_{V}$ 110], attributed to the suppression of cation ordering and delocalization of electrons with increasing pressure, produced by the increase in orbital overlap [108]. On the other hand, departures from ideal cation to oxygen stoichiometry in $\mathrm{Fe}_{3(1-\delta)} \mathrm{O}_{4}$ depress $\mathrm{T}_{V}$ down to values as low as $82 \mathrm{~K}$ [111], and beyond a critical composition $\left(\delta_{c r} \approx 0.0039\right)$ the transition changes from first to second order [106, 112 114], attributed to the inhibition of the long-range ordered structure [115].

\subsubsection{Structure and properties of epitaxial $\mathrm{Fe}_{3} \mathrm{O}_{4}$ films}

The fact that magnetite is a suitable material for application in spintronics, combined with the development of ultra-high vacuum techniques, has produced strong interest in the deposition of magnetite thin films during last years. Much effort has been done by many groups to grow and characterize high-quality epitaxial thin films using different methods such as reactive vapor deposition [16], electron beam evaporation [117], MBE [117 120], sputtering [121 123] and PLD [98, 124 128]. There are several substrates that have been used in order to grow magnetite epitaxial films such as $\mathrm{Si}, \mathrm{Al}_{2} \mathrm{O}_{3}, \mathrm{MgAl}_{2} \mathrm{O}_{4}, \mathrm{SrTiO}_{3}$ and $\mathrm{MgO}$. However, the most commonly chosen substrate is $\mathrm{MgO}(001)$, due to the small lattice mismatch with $\mathrm{Fe}_{3} \mathrm{O}_{4}(0.35 \%)$. It has been reported that the $\mathrm{Fe}_{3} \mathrm{O}_{4}$ films grow on $\mathrm{MgO}$ (100) coherently strained, relaxing partially above a critical thickness, but even for thickness of $700 \mathrm{~nm}$ the relaxation is still partial [120, 129].

It has been observed that differences exist between some properties of the bulk material and those of epitaxial magnetite films, such as the non saturation of the magnetization at high fields [129, 130], decreased and broadened Verwey transition temperature [124, 127, 128], superparamagnetic behavior of films below $5 \mathrm{~nm}$ [131, 132], increased resistivity found in epitaxial magnetite films [119, 124, 133] and large negative magnetoresistance [134-136]. 
These anomalous behaviors have often been associated to the films microstructure and the presence of structural growth defects called antiphase boundaries (APBs) [129, 130].

\section{APBs: Antiphase Boundaries}

Both $\mathrm{Fe}_{3} \mathrm{O}_{4}$ and $\mathrm{MgO}$ crystallize with the $\mathrm{O}$ atoms in an approximately facecentered cubic (fcc) lattice, and the similar $d$ spacings of the $\mathrm{O}$ lattices in $\mathrm{MgO}$ and $\mathrm{Fe}_{3} \mathrm{O}_{4}$ make $\mathrm{MgO}$ an excellent substrate for the growth of epitaxial single crystal $\mathrm{Fe}_{3} \mathrm{O}_{4}$.

There are two forms of symmetry breaking in $\mathrm{MgO} / / \mathrm{Fe}_{3} \mathrm{O}_{4}$ epitaxy. The first one is due to the fact that $\mathrm{Fe}_{3} \mathrm{O}_{4}$ has twice the unit cell size of $\mathrm{MgO}$, which means that, when two growing islands meet, the correct stacking sequence of the spinel structure can occur as well as the stacking sequence being half a unit cell (Figure 2.3), and structural defects can be formed during the growth process: antiphase boundaries. The other one is due to the fact that the $\mathrm{Fe}_{3} \mathrm{O}_{4}$ $(\mathrm{Fd} \overline{3} \mathrm{~m})$ crystal structure is lower in symmetry than $\mathrm{MgO}(\mathrm{Fm} 3 \mathrm{~m})$ structure. Therefore, $\mathrm{MgO}$ unit cell can be rotated by $90^{\circ}$ around an axis perpendicular to the interface, whereas $\mathrm{Fe}_{3} \mathrm{O}_{4}$ cannot, which leads to the formation of APBs. Across the APBs the oxygen lattice remains undisturbed while the cation lattice is displaced, therefore, the system remains monocrystalline.

Although it was first believed that the APBs formed at the very first stages of the growth and that the domain size was fixed [131], Eerenstein et al. demonstrated that the density of APBs depends directly on the deposition time during film growth [133, 137], decreasing systematically with increasing deposition time. Therefore, the antiphase domain sizes (D) are bigger in the thickest magnetite films due to the prolonged deposition time $t$ [119]; indeed, D increases with the increase of film thickness as:

$$
D \propto \sqrt{t} \Rightarrow D=D_{0} \sqrt{t}
$$

Finally, it has been accepted that the density of the APBs is strongly influenced by the growth conditions and substrate microstructure 131, 138, 139]. Indeed, it has been observed the existence of $\mathrm{APBs}$ in $\mathrm{Fe}_{3} \mathrm{O}_{4}$ films grown on $\mathrm{MgAl}_{2} \mathrm{O}_{4}$ and related to the observation of the absence of strain relaxation [140]. If the APBs formed lead to the formation of areas with opposite sign of stress, the stress compensation reduces the effective stress experienced by the films. Thus, APBs can be considered as another stress-relaxation mechanism [120, 140]. 


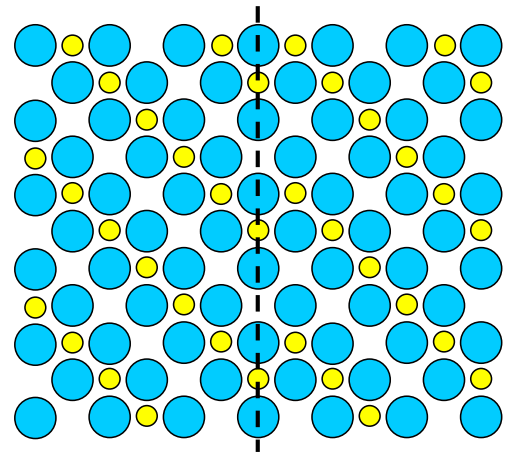

(a)

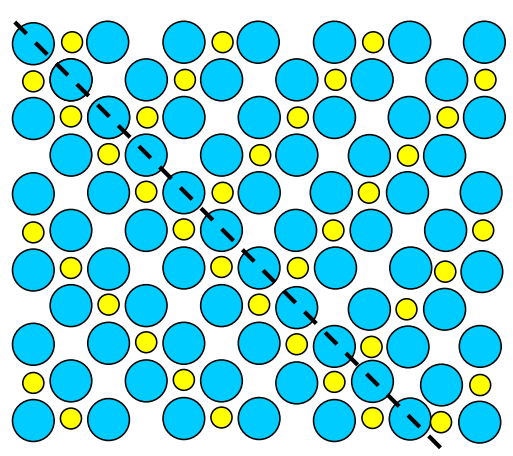

(c)
APB

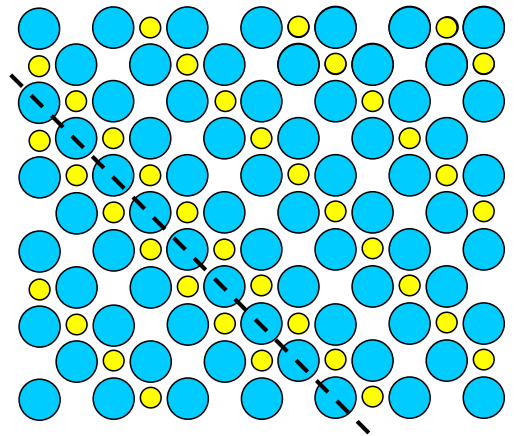

(b)

APB

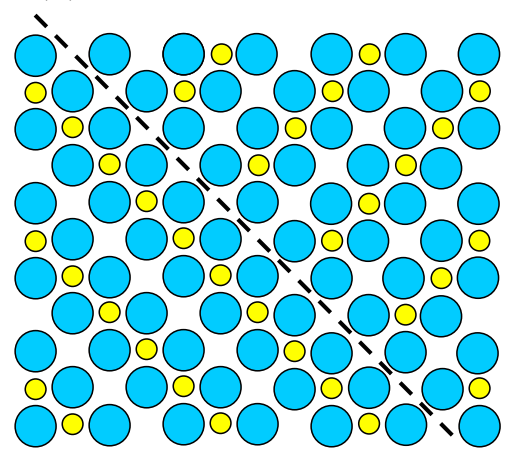

(d)

APB

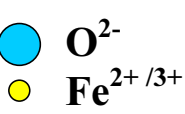

Figure 2.3: Schematic representation of an APB in $\mathrm{Fe}_{3} \mathrm{O}_{4}$ formed in a $\mathrm{MgO}$ (001) substrate assuming a perfect oxygen sublattice: (a) with $1 / 2$ [100] shift vector along the [010] direction; (b) with $1 / 4$ [100] shift vector along the [11̄0] direction; (c) rotationaltype APB aligned along [110] with higher coordination for anions at the boundary; and (d) rotational-type APB aligned along [110] with lower coordination for anions at the boundary [120].

In bulk magnetite the dominating magnetic coupling is the antiferromagnetic superexchange between neighboring tetrahedral and octahedral cation sites, and the coupling between octahedral site cations is effectively ferromagnetic. However, in $\mathrm{Fe}_{3} \mathrm{O}_{4}$ thin films, these magnetic interactions are altered at the APBs [130]. Across the boundary plane there are new, strong $180^{\circ}$ Fe-O-Fe superexchange paths between octahedral site cations, which does not exist in bulk $\mathrm{Fe}_{3} \mathrm{O}_{4}$. The result is that, across the boundary the intrasublattice exchange interactions dominate, which reverse the spin couplings, and the structural boundary then separate oppositely magnetized regions. Therefore, the resultant coupling between two domains turns out to be either frustrated or antiferromagnetic. 
Only when two islands with matching orientations meet, the resultant coupling between the two domains is ferromagnetic [141]. However, this event has a probability of only $1 / 8$ and equivalent to the formation of one larger domain out of two smaller ones, without an internal boundary.

APBs do not all act as magnetic domain walls, which has been demonstrated by a comparison between atomic and magnetic force microscopy images, showing a larger magnetic domain size than the antiphase one [123]. The antiferromagnetic coupling model by Margulies et al. [130] is the most extended in literature in order to explain magnetite films anomalous behavior:

- Decreased saturation magnetization:

The APBs play an important role in the properties of magnetite thin films, as its presence leads to new magnetic exchange interactions, which are not present in the bulk material. The exchange interactions occurring across the APBs, predominantly antiferromagnetic, have been identified as the reason for the impossibility to saturate the film in moderate magnetic fields, as the APBs separate oppositely magnetized regions [129, 130]. Therefore, the absence of saturation in high fields should be independent of deposition technique, as found by other authors [116, 119, 121, 142], and is a volume rather than a surface effect [121|. The approach to saturation in high fields follows the equation 2.4 [129]:

$$
M=M_{S}\left(1-b / H^{n}\right)
$$

where $\mathrm{M}$ is the magnetization, $\mathrm{M}_{S}$ the saturation value, $\mathrm{b}$ a parameter related to the density of APBs which varies with film thickness (t) as $\mathrm{t}^{-0.15}$ [143], and $\mathrm{n}$ an exponent equal to 0.5 in this model.

For a given applied field, $\mathrm{H}$, the magnetization projection along $\mathrm{H}$ reaches a minimum value at each $\mathrm{APB}$. The effective APB width decreases when the applied field increases [144], and becomes equal to the crystallographic width (two crystallographic planes) when the applied field is infinite. However, for finite applied fields, the width of the APB might not be negligible compared to the distance between two APBs (see figure 2.4). The measured magnetization is therefore necessarily lower than the bulk one, due to this decrease of the magnetization at the APB. Regarding the $\mathrm{M}_{S}$ value extrapolated at infinite field, is usually lower than the bulk value of $4.1 \mu_{B} / f . u$.. It is known that magnetic saturation moment should be intrinsic to the $\mathrm{Fe}_{3} \mathrm{O}_{4}$. However, it is still not understood in which way the APB density might influence the $\mathrm{M}_{S}$ value. 


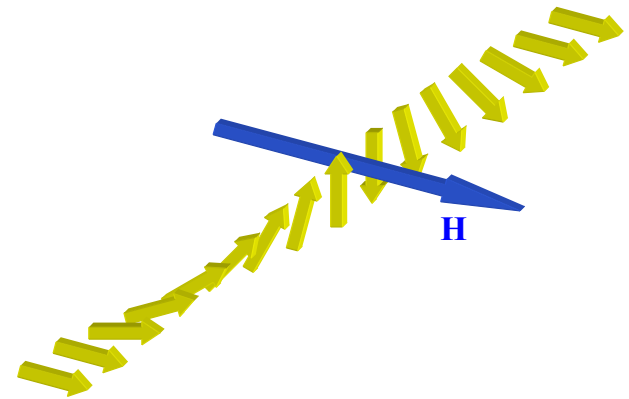

Figure 2.4: Spin - orientation of two ferromagnetic chains with antiferromagnetic coupling at an atomically sharp boundary subject to a magnetic field [134].

- Superparamagnetism in ultra thin films:

A superparamagnetic behavior has been reported [131, 132] for very thin films ( $\mathrm{t}<5 \mathrm{~nm}$ ), which has been ascribed to thermal fluctuations of the magnetic moment of the structural domains defined by APBs. Superparamagnetism is possible only if there is antiferromagnetic coupling between APBs. Otherwise, if all couplings were ferromagnetic, a domain would be rigidly locked, and superparamagnetism would not be observed. Antiferromagnetic coupling leads to frustration, as the superexchange barriers effectively cancel each other, enabling the domains to fluctuate much more freely and thus the film becomes superparamagnetic. Indeed, it has been found that the superparamagnetic behavior can be tuned by varying the structural domain size of the films, by means of annealing or using different substrates or buffer layers [132]. The thickness ( $t$ ) dependence of the barrier was empirically shown to be $t^{1 / 3}$.

- Decreased Verwey transition temperature:

A broadened and shifted $\mathrm{T}_{V}$ to lower temperatures with decreasing film thicknesses has been reported for magnetite films [124, 127, 128]. Different explanations have been given, such as the presence of cation vacancies, oxygen non-stoichiometry [118] like in bulk magnetite ( 90,106$]$, see section 2.1.1), residual strain resulting from the lattice mismatch with the substrate [122, 124, 126, 128], or difference in thermal expansion coefficients between substrate and the $\mathrm{Fe}_{3} \mathrm{O}_{4}$ thin film 145]. However, it could be due to the presence of defects such as APBs [119, 125]: a decrease of the antiphase domain size in the thinner films might affect the formation of the charge localization responsible for the conducting mechanism in $\mathrm{Fe}_{3} \mathrm{O}_{4}$ (section 2.1.1) hindering the long-range charge order, therefore inhibiting the Verwey transition. This effect may explain the shift in $\mathrm{T}_{V}$ or even its disappearance in the case of the thinnest films, where the APB density is highest [125, 146]. 
- APBs as the origin of the increased resistivity in epitaxial films:

An increase in resistivity with decreasing $\mathrm{Fe}_{3} \mathrm{O}_{4}$ film thickness has been observed [119, 124, 133] and explained by the enhancement of the APB density [130, 134, 135]. As it happens with any structural defect, the APBs act as scattering centers which hinder electron transport across the films, and as the magnetic coupling across an APB has been reported to be antiferromagnetic, the charge transfer is inhibited since the transport in $\mathrm{Fe}_{3} \mathrm{O}_{4}$ by electron hopping requires a ferromagnetic alignment between neighboring iron atoms in the octahedral sites, therefore, increasing the resistivity.

Eerenstein et al. [133] used the effective medium approximation for a two-phase composite to describe the conductivity of the films, which is a function of both the bulk and boundary conductivities and of the fraction in which both phases are present.

In this model, $\phi_{b}$ and $\phi_{A P B}$ are the volume fractions of the bulk and APB phases respectively, such that $\phi_{b}+\phi_{A P B}=1$. The domains are assumed to be square shaped with an average size D and a domain boundary of thickness d. Thus, $\phi_{b}$ is calculated as:

$$
\phi_{b}=\frac{(D-d)^{2}}{D^{2}}=\left(1-\frac{d}{D}\right)^{2}
$$

If $\sigma_{b}$ is the bulk conductivity and $\sigma_{A P B}$ is the boundary conductivity, the effective conductivity $\sigma_{e}$ of the magnetite film is given by:

$$
\begin{aligned}
\sigma_{e} & =0,5 \cdot\left[\left(\sigma_{b}-\sigma_{A P B}\right)\left(2 \phi_{b}-1\right)\right. \\
& +\sqrt{\left.\left(\sigma_{b}^{2}-\sigma_{A P B}^{2}\right)\left(2 \phi_{b}-1\right)^{2}+2 \sigma_{b} \sigma_{A P B}\left(2-\left(2 \phi_{b}-1\right)^{2}\right)\right]}
\end{aligned}
$$

Finally, if $\sigma_{A P B} \ll \sigma_{b}$, and as long as $\phi_{b}>0.5$, equation 2.6 may be approximated as:

$$
\sigma_{e}=\sigma_{b}\left(2 \phi_{b}-1\right)
$$

- Increased negative magnetoresistance in magnetite thin films:

Negative magnetoresistance has been found in $\mathrm{Fe}_{3} \mathrm{O}_{4}$ films grown on MgO substrates [134-136]. This behavior is thought to be mainly due to the APBs presence [119], which produces that, although the resistivity in magnetite films follows the bulk behavior within the ferromagnetic domains defined by the APBs, it is strongly enhanced across the antiferromagnetically coupled APBs, where the transport is dominated by tunneling [147]. By applying a magnetic field, the antiparallel spins will align themselves to some degree with the magnetic field, thus increasing the electron transport across the boundaries [130, 134], as shown in figure 2.4 . 


\subsection{Growth optimization}

In this section, we carefully explain the different steps necessary in order to obtain high-quality magnetite epitaxial thin films.

Epitaxial $\mathrm{Fe}_{3} \mathrm{O}_{4}$ thin films with thicknesses of around $45 \mathrm{~nm}$ were grown on $\mathrm{MgO}$ (001) substrates by means of the PLD system described in section 1.1, by using a stoichiometric polycrystalline sintered target. In order to optimize the growth parameters, commercial monocrystalline $\mathrm{MgO}$ (001) substrates were chosen, due to the 2:1 relation between its lattice parameter $\left(a_{M g O}=4.213\right.$ $\dot{A})$ and magnetite lattice parameter $\left(a_{\mathrm{Fe}_{3} \mathrm{O}_{4}}=8.3967 \dot{A}\right)$, which produces a misfit as low as $0.35 \%$. Commercial one-side-polished substrates from Crystal GmbH company were used, with dimensions $5 \times 5 \times 0.5 \mathrm{~mm}^{3}$ and nominal roughness $<0.5 \mathrm{~nm}$.

The substrates were attached with silver paste on the substrate holder and dried for ten minutes at $40{ }^{\circ} \mathrm{C}$ in air atmosphere. After introducing the substrate in the process chamber, the chamber is pumped down to a base pressure lower than $10^{-8}$ torr. Afterwards, the substrate is heated up to $650{ }^{\circ} \mathrm{C}$ at $10^{\circ} \mathrm{C} / \mathrm{min}$ and allowed to degas for $30 \mathrm{~min}$., and then the deposition temperature is set at $10^{\circ} \mathrm{C} / \mathrm{min}$. After growing the thin film, the substrate temperature is decreased at a rate of $10{ }^{\circ} \mathrm{C} / \mathrm{min}$ to room temperature and the sample is removed from the deposition chamber.

The main parameters which determine the quality of the $\mathrm{Fe}_{3} \mathrm{O}_{4}$ films are the process gas atmosphere, the deposition rate, and the substrate temperature. As the PLD method essentially preserves the stoichiometry from the target to the deposited film for simple oxides, working in ultra high vacuum is suitable in the case of the magnetite growth. Therefore, the effect of the deposition rate was studied in first place, as it can influence both the crystalline quality of the film and its stoichiometry: too low deposition rates may produce an oxidation of the deposited material, performing an in situ reactive PLD process. Afterwards, all the deposition conditions were maintained except the substrate temperature, in order to find the optimal temperature for the epitaxial growth of magnetite on $\mathrm{MgO}$ (001).

\subsubsection{Target preparation}

Target materials play a leading role in the PLD method, a control of their high stoichiometric quality being essential. In order to obtain the desired $\mathrm{Fe}_{3} \mathrm{O}_{4}$ stoichiometry, pure spherical iron powder (99.9\% purity) with a particle size less than $10 \mu \mathrm{m}$ diameter was used for the target preparation by solid-state reaction. 
Several thermal treatments were performed in order to obtain the desired stoichiometry: oxidation in air atmosphere and reduction in $\mathrm{CO} / \mathrm{CO}_{2}$ atmosphere [148]. Iron oxidation was produced by annealing the iron powder at $775{ }^{\circ} \mathrm{C}$ for 7 hours in air atmosphere, the treatment was repeated several times grounding the sample in between in order to homogenize the powder and therefore improve the oxidation. After powdering the sample, it was uniaxially pressed into a pellet with a $1.60 \mathrm{~cm}$ diameter mold to form the target, and an annealing was performed at $1425{ }^{\circ} \mathrm{C}$ for 12 hours in $\mathrm{CO} / \mathrm{CO}_{2}$ atmosphere at a 4:96 ratio, obtaining a stoichiometric $\mathrm{Fe}_{3} \mathrm{O}_{4}$ target.

Structural and magnetic characterization of the $\mathrm{Fe}_{3} \mathrm{O}_{4}$ target were performed by means of XRD and SQUID magnetometry, respectively, in order to guarantee its quality before starting the thin film growth process.

\section{Target structural characterization}

The XRD measurement was performed at room temperature and the data was analyzed by the Rietveld method by means of FullProf (figure 2.5). The analysis demonstrates that there is only crystalline $\mathrm{Fe}_{3} \mathrm{O}_{4}$, without any parasitic phases such as $\mathrm{Fe}_{2} \mathrm{O}_{3}, \mathrm{FeO}$ of metallic $\mathrm{Fe}$, which could have remained after the sinterization process.

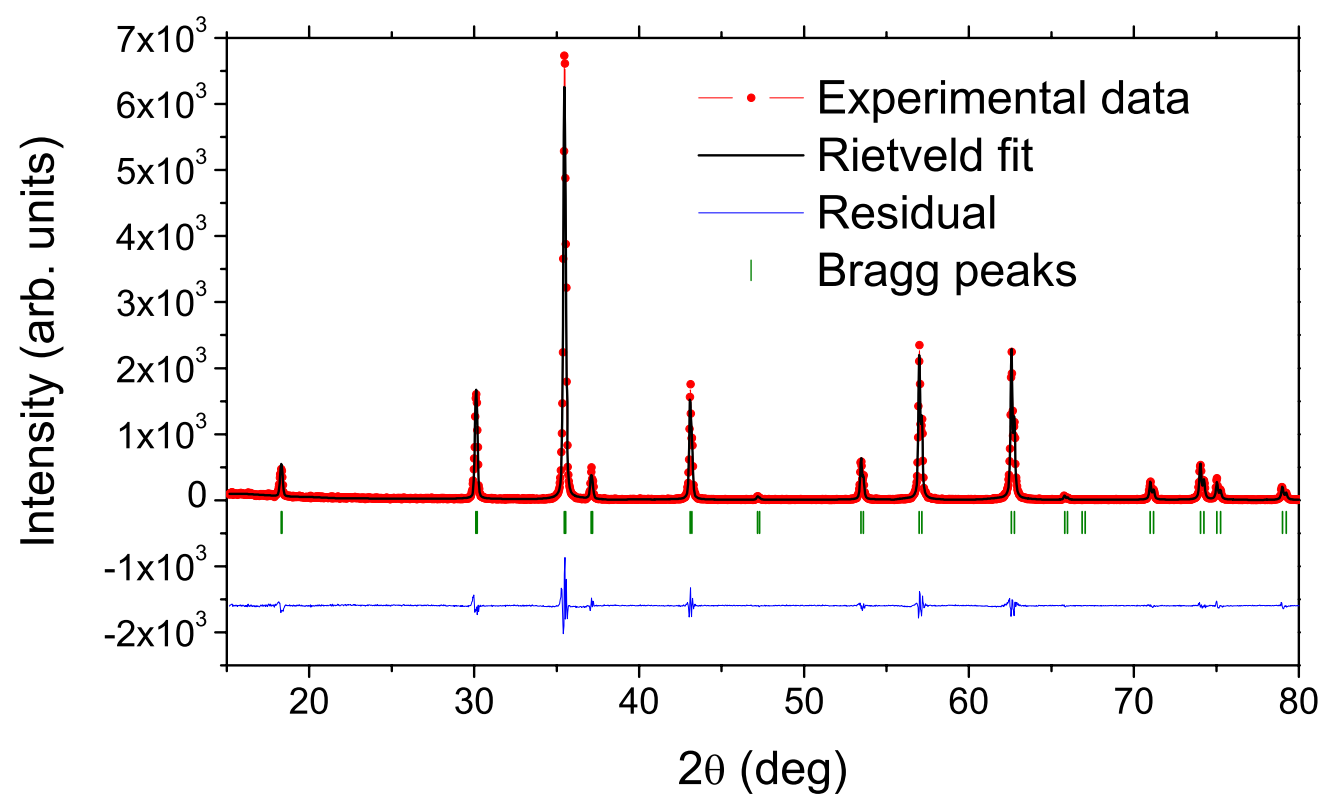

Figure 2.5: XRD measurement $(\theta-2 \theta)$ from the sinterized $\mathrm{Fe}_{3} \mathrm{O}_{4}$ target. The black line represents the Rietveld refinement, and the blue line indicates the residual. 


\section{Target magnetic characterization}

The target magnetization was measured by SQUID magnetometry as a function of the applied magnetic field (Figure 2.6) and as a function of the temperature (Figure 2.7).

The hysteresis loop at a temperature of $10 \mathrm{~K}$ shows a magnetization saturation of $4 \mu_{B} / f . u$., which coincides with the bulk value, confirming the homogeneity and high quality of the target.
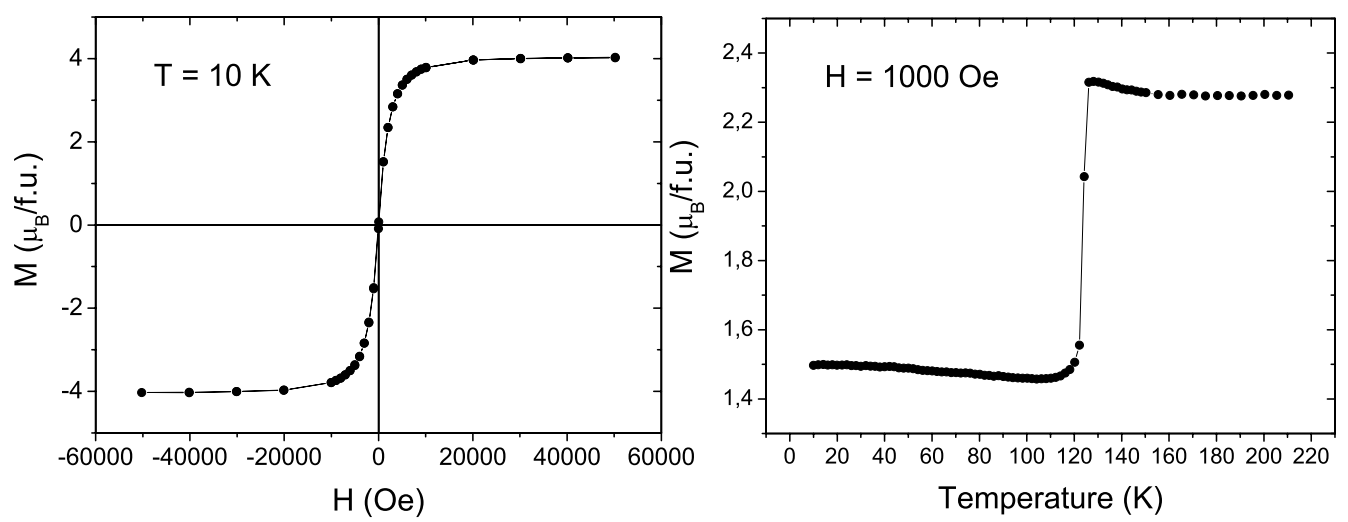

Figure 2.6: Hysteresis loop of an opti- Figure 2.7: Magnetization as a function mized magnetite target at $10 \mathrm{~K}$.

of the temperature in an applied field magnetic field of 1000 Oe.

From the magnetization measurements as a function of the temperature in an applied magnetic field of 1000 Oe (Figure 2.7), the temperature of the Verwey transition was found to be $123 \mathrm{~K}$. This value coincides with the bulk value, and as the Verwey transition is extremely sensible to stoichiometry (see section 2.1), the $\mathrm{Fe}_{3} \mathrm{O}_{4}$ target quality is demonstrated.

Once the target quality is achieved, it can be loaded into the PLD system and proceed with the film deposition optimization process.

\subsubsection{Deposition rate}

In first place, a study of the deposition rate influence on the magnetite films quality was performed in order to find the optimal growth rate for magnetite deposition in our system.

With this purpose, magnetite films were deposited in ultra-high vacuum at three different deposition rates: $3 \AA / \mathrm{min}, 36 \AA / \mathrm{min}$ and $90 \AA / \mathrm{min}$. 
The number of laser pulses was adjusted in order to obtain films with thicknesses around $45 \mathrm{~nm}$, and the substrate temperature was chosen to be $450{ }^{\circ} \mathrm{C}$, in agreement with the parameters used by other authors [98, 124 128]. Afterwards, the films were structurally characterized by means of XRD (Figure 2.8).

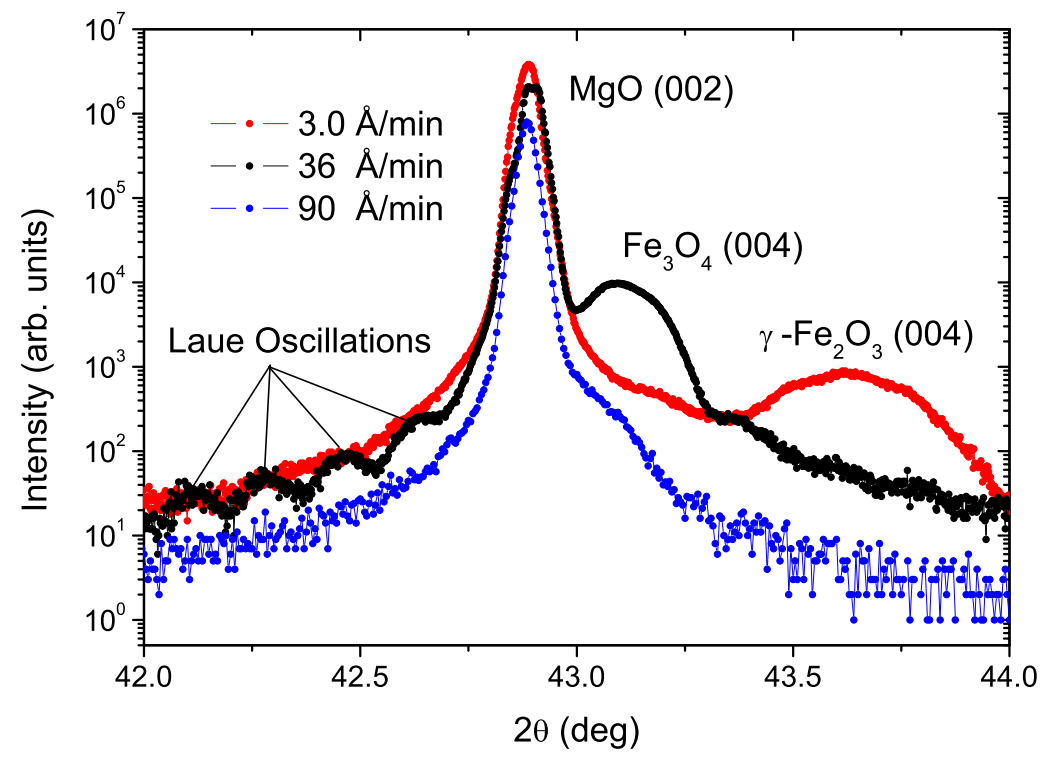

Figure 2.8: XRD measurements $(\theta-2 \theta)$ from samples growth at different deposition rates. Substrate temperature was chosen to be $450{ }^{\circ} \mathrm{C}$.

The symmetrical $\theta-2 \theta$ scan around the (002) Bragg peak from the $\mathrm{MgO}$ substrate performed on the film deposited at the lower deposition rate (3 $\AA /$ min.) show the appearance of the $\gamma-\mathrm{Fe}_{3} \mathrm{O}_{4}$ (maghemite) peak, indicating that the film has been oxidized during the growth process and crystalline maghemite has been obtained instead of crystalline magnetite. However, the diffractrogram of the film deposited at the higher rate $(90 \AA / \mathrm{min}$.) does not show maghemite, but the $\mathrm{Fe}_{3} \mathrm{O}_{4}(004)$ diffraction peak almost disappears, implying a poor crystalline quality. Finally, the diffraction pattern of the sample deposited at $36 \AA /$ min., shows the $\mathrm{Fe}_{3} \mathrm{O}_{4}(004)$ peak together with Laue oscillations (due to finite size effects), proving the high structural coherence along the full film thickness and the crystalline quality of the film.

Therefore, the optimal deposition rate is found to be $36 \AA / \mathrm{min}$ at a deposition temperature of $450{ }^{\circ} \mathrm{C}$, which provides high crystalline quality $\mathrm{Fe}_{3} \mathrm{O}_{4}$ films. 


\subsubsection{Substrate temperature}

Once the deposition rate is optimized, the influence of the substrate temperature $\left(\mathrm{T}_{S}\right)$ on the epitaxial growth of magnetite on $\mathrm{MgO}(001)$ is studied. With this purpose, magnetite thin films (45 nm thickness) were grown at different temperatures ranging from $300{ }^{\circ} \mathrm{C}$ to $800{ }^{\circ} \mathrm{C}$ on $\mathrm{MgO}(001)$ substrates at the optimized deposition rate (36 $\AA$ /min., see section 2.2 .2$)$, and structural and magnetic characterization was performed.

The structural characterization of the films deposited at different temperatures was performed by means of XRD (see figure 2.9). The symmetrical $\theta-2 \theta$ scan around the (002) Bragg peak from the $\mathrm{MgO}$ substrate performed on the film deposited at the lower substrate temperature $\left(350{ }^{\circ} \mathrm{C}\right)$ show the absence of the $\mathrm{Fe}_{3} \mathrm{O}_{4}(004)$ peak, indicating a poor crystalline quality. However, the $\mathrm{Fe}_{3} \mathrm{O}_{4}(004)$ peak is visible in the diffraction patterns of the samples grown at $450{ }^{\circ} \mathrm{C}$ and $550{ }^{\circ} \mathrm{C}$ and surrounded by the Laue oscillations, which proves the high crystalline quality of the magnetite films.

Therefore, from the crystalline point of view, the epitaxial growth of magnetite on the $\mathrm{MgO}$ (001) substrates takes place at a minimum substrate temperature of $450{ }^{\circ} \mathrm{C}$.

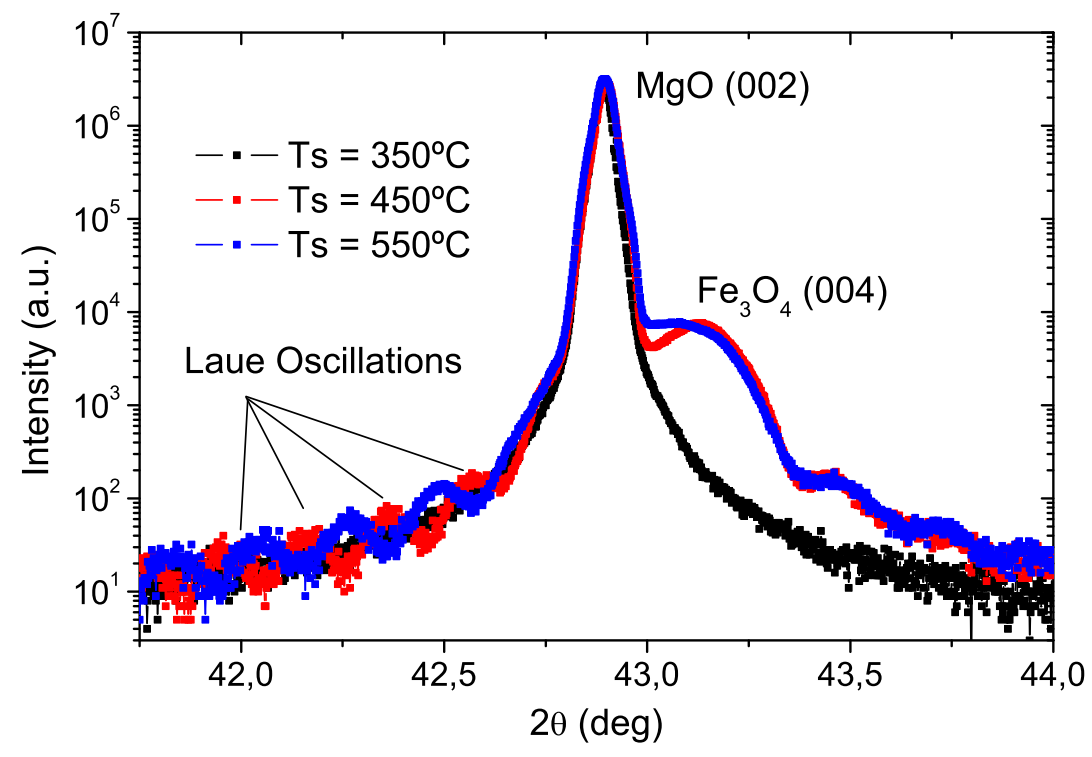

Figure 2.9: XRD measurements $(\theta-2 \theta)$ from samples grown at different substrate temperatures. 
The influence of the deposition temperature in the stoichiometry of the films can be observed in the Verwey Transition temperature $\left(\mathrm{T}_{V}\right)$ and in the abruptness of the transition. SQUID magnetometry measurements were performed as a function of increasing temperature in an applied magnetic field of 500 Oe (figure 2.10). All samples were zero-field cooled prior to measurement.

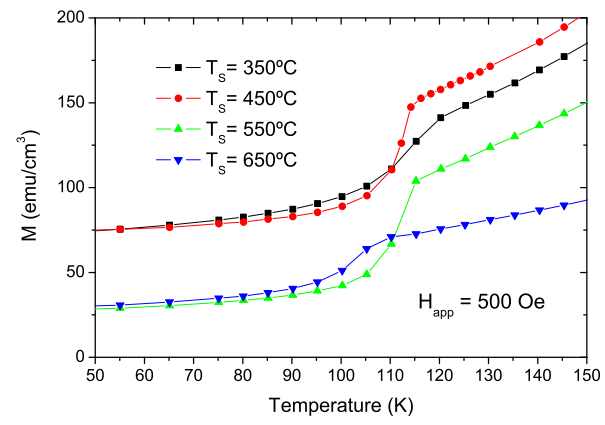

Figure 2.10: Magnetization as a function of temperature, for $\mathrm{Fe}_{3} \mathrm{O}_{4}$ films deposited at different substrate temperatures $\mathrm{T}_{S}$.

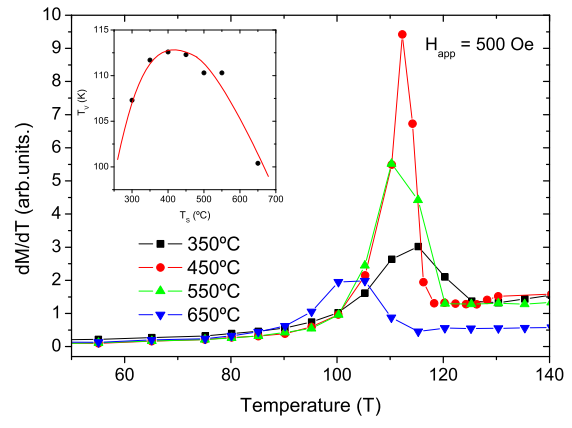

Figure 2.11: Derivative of the magnetization as a function of the temperature, for $\mathrm{Fe}_{3} \mathrm{O}_{4}$ films deposited at different substrate temperatures.

It is easier to appreciate $\mathrm{T}_{V}$ and the abruptness of the transition if we perform a derivative of the magnetization as a function of temperature (figure 2.11). The most abrupt Verwey transition with the highest derivative peak is found for the sample growth at $450{ }^{\circ} \mathrm{C}$, and regarding the temperature transition, it can be seen that, as the deposition temperature is increased, $\mathrm{T}_{V}$ increases and find its maximum value $112 \mathrm{~K}$ at $\mathrm{T}_{S}=350{ }^{\circ} \mathrm{C}-450{ }^{\circ} \mathrm{C}$ whereas for $\mathrm{T}_{S}>450{ }^{\circ} \mathrm{C}, \mathrm{T}_{V}$ decreases again. Therefore, the nearest value to the reported bulk transition temperature and the most abrupt transition is found for the film growth with substrate temperature of $\mathrm{T}_{S}=450^{\circ} \mathrm{C}$.

Magnetic hysteresis loops have also been measured in a maximum magnetic field of $5 \mathrm{~T}$ at room temperature. If we observe the magnetization values at the maximum field (figure 2.12) it can be seen that the films magnetization increase with increasing substrate temperature, find a maximum for $\mathrm{T}_{S}=450^{\circ} \mathrm{C}$ and then decrease again.

From the structural and magnetic characterization, the optimal substrate temperature to grow magnetite films in our system is confirmed to be $450{ }^{\circ} \mathrm{C}$. After having found the optimized growth parameters for magnetite thin films (table 2.1), a film with a thickness of approximately $40 \mathrm{~nm}$ was produced and exhaustively characterized. 


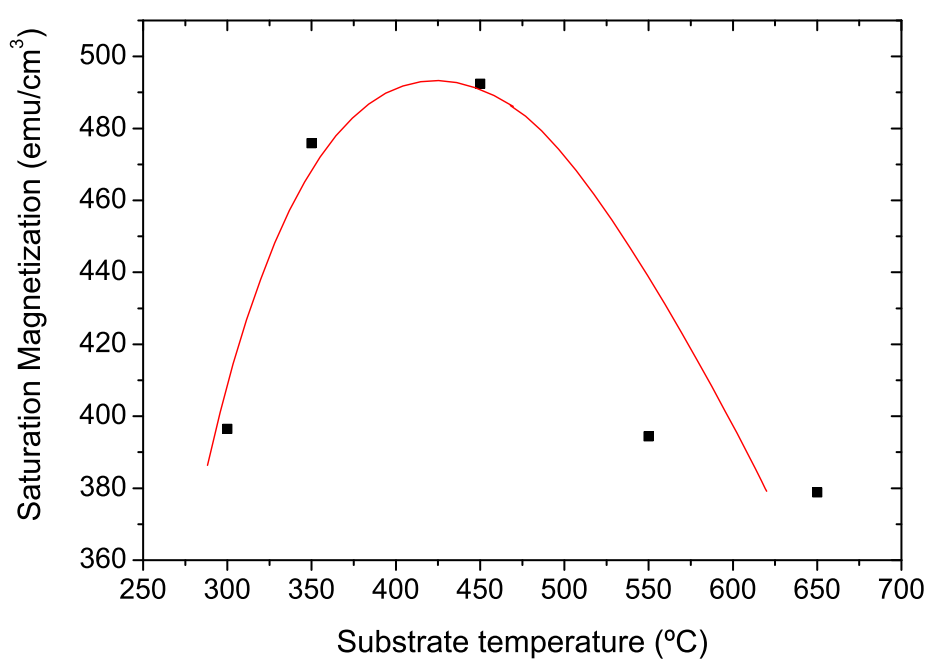

Figure 2.12: Saturation magnetization from samples grown at different substrate temperatures measured with an applied magnetic field of $5 \mathrm{~T}$ at room temperature.

\begin{tabular}{||c|c|c||}
\hline \hline Process atmosphere & Deposition rate & Substrate temperature \\
\hline Ultra high vacuum & $36 \AA / \mathrm{min}$ & $450{ }^{\circ} \mathrm{C}$ \\
\hline \hline
\end{tabular}

Table 2.1: Optimized deposition parameters for magnetite film growth.

\subsubsection{Structural characterization}

The symmetrical $\theta-2 \theta$ scan around the (002) Bragg peak from the $\mathrm{MgO}$ substrate shows the $\mathrm{Fe}_{3} \mathrm{O}_{4}(004)$ reflection and its Laue oscillations up to $10^{\text {th }}$ order (figure 2.13). As the Laue oscillations have its origin in the finite number of diffractive layers, from their periodicity $\Delta q$, a coherence length $\xi=2 \pi / \Delta q=$ $47 \pm 0.5 \mathrm{~nm}$ can be determined (figure 2.14). The fFWHM of the rocking curve centered at the $\mathrm{Fe}_{3} \mathrm{O}_{4}(004)$ peak is 0.04 degrees, which evidences the high crystalline quality of the magnetite samples, as the FWHM of the MgO (002) is $<0.1$ degrees.

In order to measure the film thickness independently from its crystalline quality, XRR measurements (figure 2.15) were carried out. From the numerical analysis of the spacing between the Kiessig fringes, the film thickness was found to be $41.4 \pm 0.1 \mathrm{~nm}$ within a $\pm 0,2 \%$ estimated accuracy, a value which is similar to the obtained from the Laue oscillations, indicating the crystalline coherence along the full thickness of the film. 

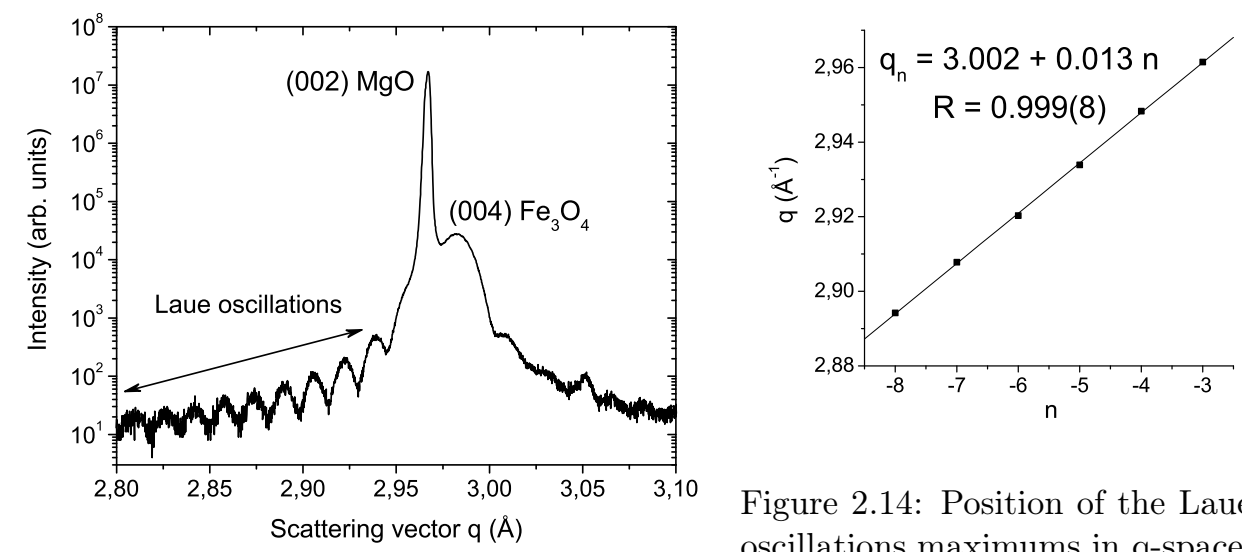

Figure 2.14: Position of the Laue oscillations maximums in q-space. From the linear fit, the coher-

Figure 2.13: $\theta-2 \theta$ from a $40 \mathrm{~nm}$ thick film grown at the optimal growth parameters. ence length can be obtained: $\xi=$ $2 \pi / \Delta q$.

From the numerical fit of the XRR data the roughness $(\sigma)$ from the magnetite film, the interface of the film with the substrate, and also the magnetite density are obtained (table 2.2). The film roughness is in the order of the nominal substrate roughness value, revealing the smoothness of the magnetite layer, and the film density is close to the bulk value. Possible differences between film and bulk density could be explained as a film lattice distortion due to epitaxial strain (see the thesis introduction).

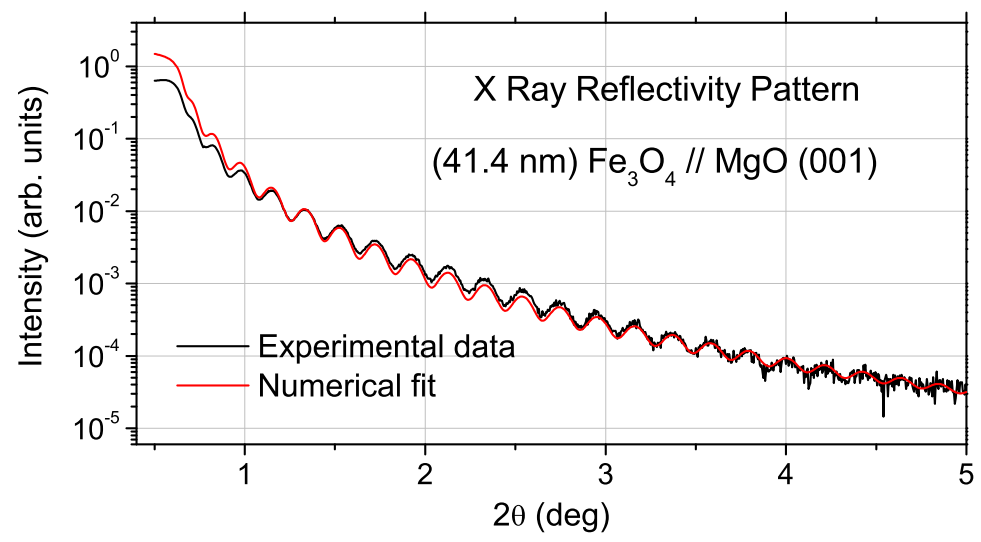

Figure 2.15: XRR measurement from a $40 \mathrm{~nm}$ thick sample produced at the optimal growth parameters. The red line corresponds to the numerical fit. 


\begin{tabular}{||c|c|c|c||}
\hline \hline Material & Thickness $(\mathrm{nm})$ & Roughness $(\mathrm{nm})$ & Density $\left(\mathrm{g} / \mathrm{cm}^{3}\right)$ \\
\hline $\mathrm{Fe}_{3} \mathrm{O}_{4}$ & 41.35 & 0.01 & 5.24 \\
\hline $\mathrm{MgO}$ & Substrate & 0.50 & 3.57 \\
\hline \hline
\end{tabular}

Table 2.2: Values obtained from the XRR numerical fit in figure 2.15,

The out-of-plane lattice parameter can be extracted from the $\mathrm{Fe}_{3} \mathrm{O}_{4}(004)$ peak position at the symmetrical XRD measurement (figure 2.13), obtaining:

$$
c_{\text {out }}\left(\mathrm{Fe}_{3} \mathrm{O}_{4}\right)=8.38 \AA
$$

From the RSM near the asymmetrical reflection (113) from the $\mathrm{MgO}$ and (226) from the $\mathrm{Fe}_{3} \mathrm{O}_{4}$, both in- and out-of-plane lattice parameters can be extracted (figure 2.16), as the scattering vector is inversely proportional to the lattice parameters:

$$
c_{\text {out }}\left(\mathrm{Fe}_{3} \mathrm{O}_{4}\right)=8.38 \AA \quad a_{\text {in }}\left(\mathrm{Fe}_{3} \mathrm{O}_{4}\right)=2 \times a_{\text {in }}(\mathrm{MgO})=8.42 \AA
$$

The lattice parameters obtained with both RSM and XRD are coherent. Due to the epitaxial growth, the magnetite in-plane lattice parameter is exactly the same as the $\mathrm{MgO}$ lattice parameter. The magnetite out-of-plane value is lower than the bulk $\mathrm{Fe}_{3} \mathrm{O}_{4}$ lattice parameter $\left(a_{\mathrm{Fe}_{3} \mathrm{O}_{4}}=8.3967 \AA\right)$, suggesting that the film is tensile strained in the plane due to the slightly larger value of the $\mathrm{MgO}$ lattice parameter with respect to the bulk $\mathrm{Fe}_{3} \mathrm{O}_{4}$ value and, therefore, compressively strained in the out-of-plane direction.

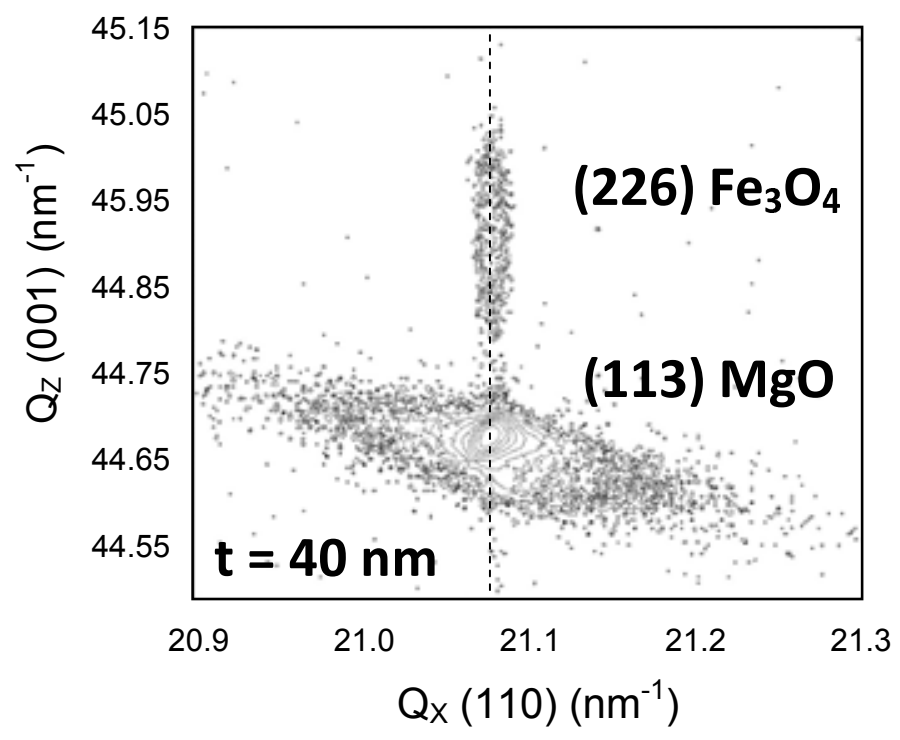

Figure 2.16: RSM near the asymmetrical reflections $\mathrm{Fe}_{3} \mathrm{O}_{4}$ (226) and $\mathrm{MgO}$ (113). 
A $\phi-$ scan of the asymmetrical $\mathrm{Fe}_{3} \mathrm{O}_{4}(226)$ reflection (figure 2.20) was measured by fixing the position angles of the sample in the $\mathrm{Fe}_{3} \mathrm{O}_{4}(226)$ reflection and rotating in-plane the sample along $360^{\circ}$. The eight peaks of similar intensity from the (311) planes were found, revealing the epitaxial in-plane orientation of the magnetite thin film: $\mathrm{MgO}[100] / / \mathrm{Fe}_{3} \mathrm{O}_{4}[100]$.

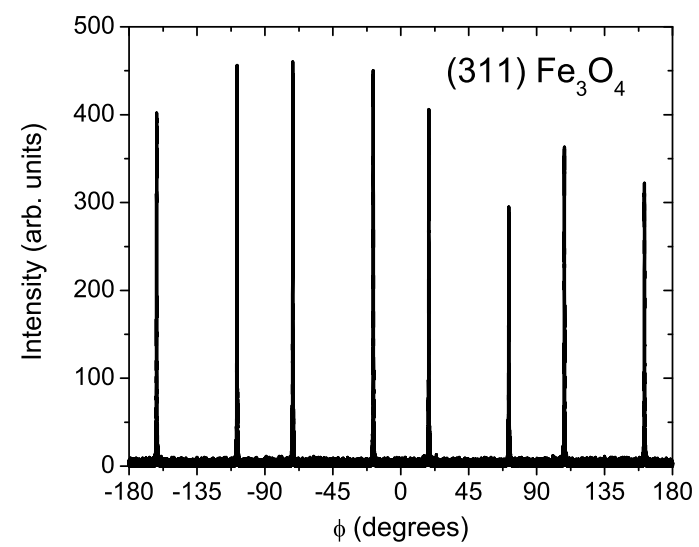

Figure 2.17: $\phi-$ scan of the asymmetrical $\mathrm{Fe}_{3} \mathrm{O}_{4}(311)$ reflection.

\subsubsection{Morphological and surface characterization}

If we want to use the magnetite film as part of a spintronic device such a magnetic tunnel junction, continuity, homogeneity and low roughness of the layer are key issues. Therefore, an exhaustive morphological and surface characterization is necessary.

The low-resolution TEM image in figure 2.18 shows that the film is continuous and quite homogeneous at lateral sizes of the order of the micron.

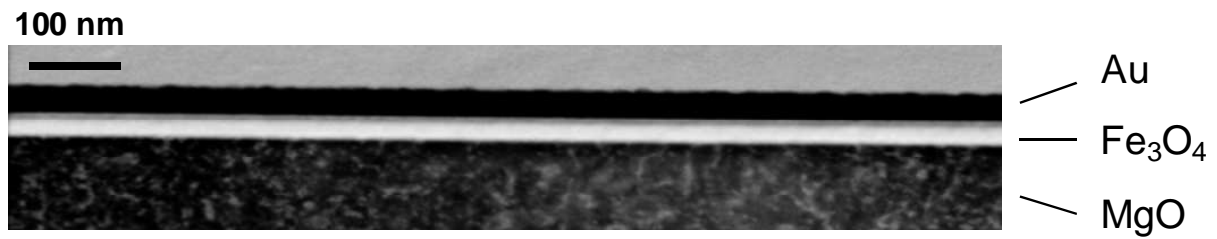

Figure 2.18: Low-resolution TEM image from a $\mathrm{MgO}(001) / /(40 \mathrm{~nm}) \mathrm{Fe}_{3} \mathrm{O}_{4} /$ (45 $\mathrm{nm}) \mathrm{Au}$ film. Measurement performed by Dr. C. Magen at the Centre d'Elaboration de Matériaux et d'Etudes Structurales, CNRS in Toulouse (France). 
High-resolution transmission electron microscopy (HRTEM) images evidence the high-quality epitaxial growth of $\mathrm{Fe}_{3} \mathrm{O}_{4}$ on $\mathrm{MgO}$, presenting a flat and smooth interface with $\mathrm{MgO}$ (Figure 2.19) and the presence of APBs.

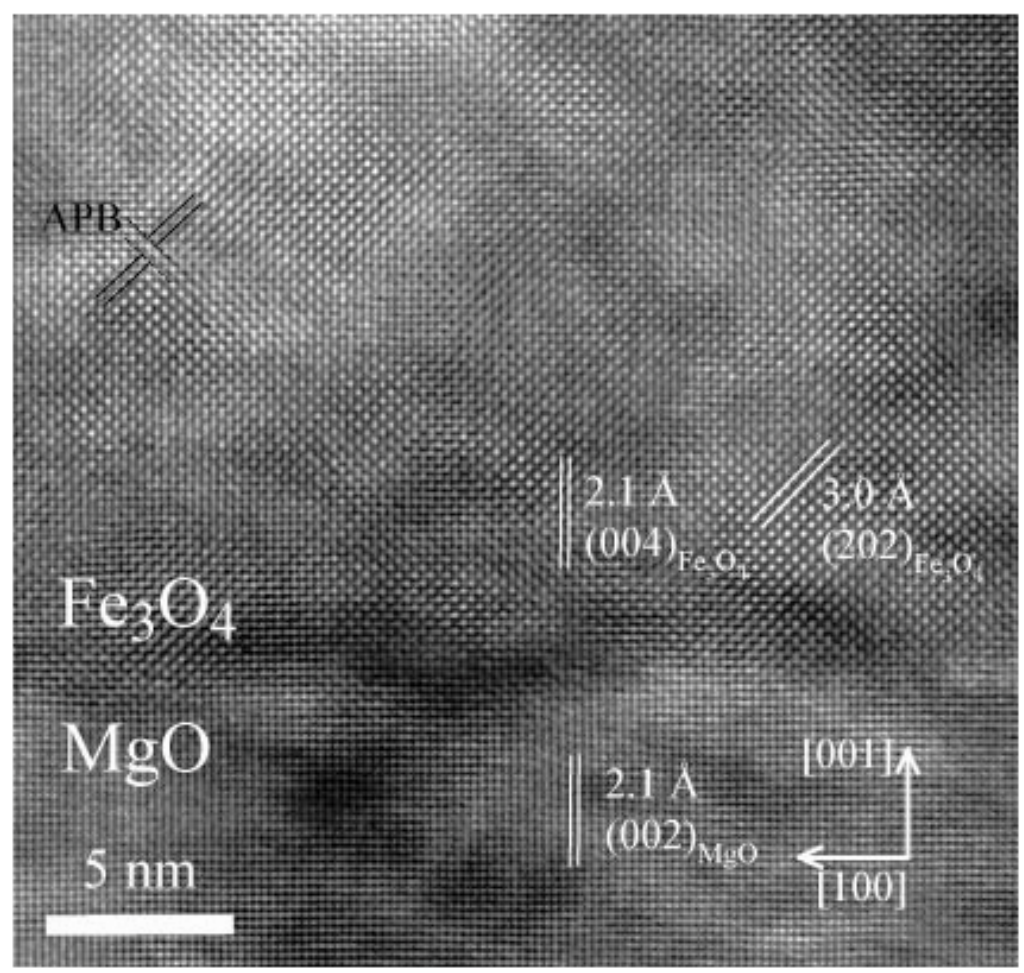

Figure 2.19: Cross section HRTEM image of a $\mathrm{MgO}(001) / / \mathrm{Fe}_{3} \mathrm{O}_{4}$ film. The different crystalline planes and distances in both $\mathrm{Fe}_{3} \mathrm{O}_{4}$ and $\mathrm{MgO}$ substrate are indicated. Measurement performed by Dr. C. Magen at the Centre d'Elaboration de Matériaux et d'Etudes Structurales, CNRS in Toulouse (France).

Electron diffraction measurements confirm the epitaxial growth and good crystallinity of the films. The diffraction image of the substrate indicates the expected cubic structure of $\mathrm{MgO}$, whereas the diffraction image of the film indicates also cubic structure of $\mathrm{Fe}_{3} \mathrm{O}_{4}$, with additional diffraction spots at intermediate positions with respect to the $\mathrm{MgO}$ image due to the doubling of the lattice parameter in $\mathrm{Fe}_{3} \mathrm{O}_{4}$. 


\section{MgO electron diffraction pattern}

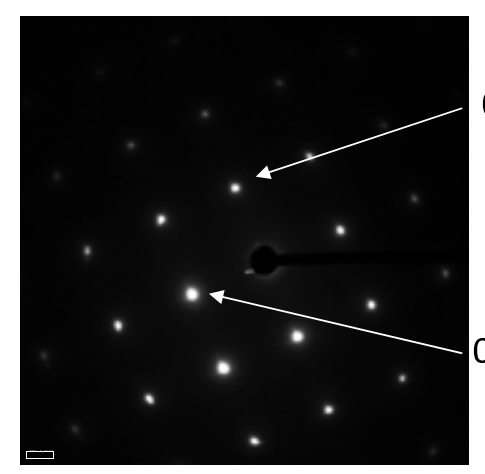

$\mathrm{Fe}_{3} \mathrm{O}_{4}$ electron diffraction pattern

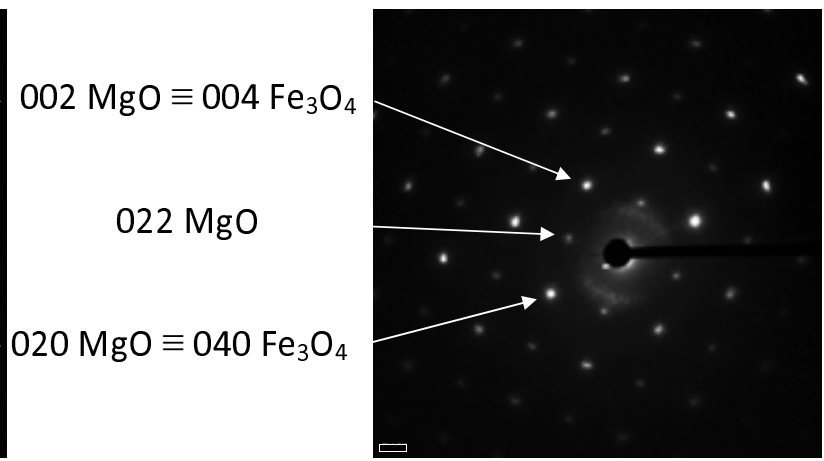

Figure 2.20: Electron diffraction measurement from the $\mathrm{Fe}_{3} \mathrm{O}_{4}$ film (right image) and the $\mathrm{MgO}$ substrate (left image). Measurement performed by Dr. C. Magen at the Centre d'Elaboration de Matériaux et d'Etudes Structurales, CNRS in Toulouse (France).

The film surface was studied by means of AFM measurements (figure 2.21) performed in tapping mode with a $300 \mathrm{kHz}$ tip, as the layer was expected to be quite flat (see section 1.2.3). Indeed, the RMS (Root-Mean-Square) roughness was found to be less than $0.1 \mathrm{~nm}$, therefore similar to the value obtained from the XRR results and as small as the substrate roughness. This is satisfactorily small in order to grow a magnetite-based heterostructure.

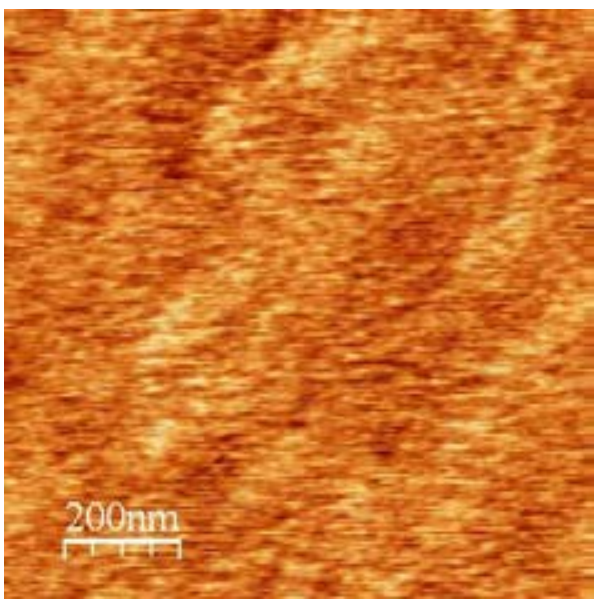

Figure 2.21: $1 \mu m \times 1 \mu m$ AFM image from a standard $40 \mathrm{~nm}$ thick magnetite film in tapping mode.

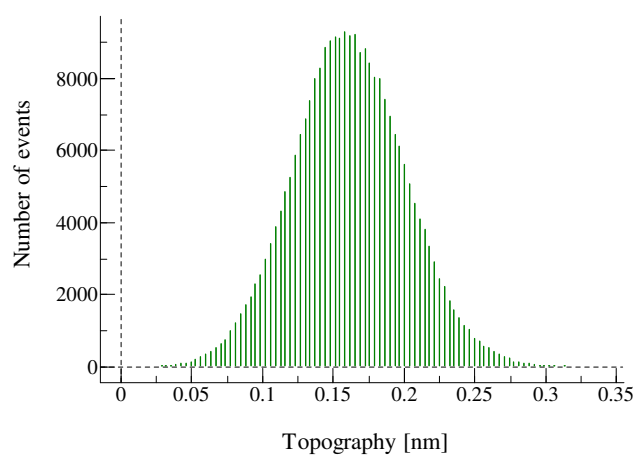

Figure 2.22: Roughness analysis histogram. The RMS average roughness of the magnetite film is $0.04 \mathrm{~nm}$. 


\subsubsection{Magnetic and electrical transport properties}

Once the structural and superficial quality of the magnetite films was confirmed, the magnetic characterization was performed by means of SQUID magnetometry. From the magnetization measurements as a function of the applied magnetic field at room temperature (figure 2.23), we obtained a value of 440 $\mathrm{emu} / \mathrm{cm}^{3}$ for the saturation magnetization, which is about $10 \%$ less than the value reported for bulk $\mathrm{Fe}_{3} \mathrm{O}_{4}$. However, this effect has been observed before in high quality magnetite films, and is generally explained due to the presence of APBs with antiferromagnetic interactions or due to the epitaxial strain (see section 2.1.2).

The Verwey Transition can be observed both in magnetization (figure 2.24) and transport measurements (figure 2.25) as a function of temperature.

In first place, and by means of SQUID magnetometry, the transition was observed in magnetization measurements (figure 2.24). The Verwey Transition Temperature was found at $110 \mathrm{~K}$, which is a value lower than the one measured in single-crystals $\left(\mathrm{T}_{V} \sim 120 \mathrm{~K}\right)$. However, a decrease of $\mathrm{T}_{V}$ has been observed by other authors in epitaxial thin films for thickness below $100 \mathrm{~nm}$, and related with epitaxial strain 122, 124, 126, 128, 145] and the presence of defects such as APBs [119, 125] (for more details see section 2.1.1).

Indeed, the existence of the transition evidences a high stoichiometry, as slight deviations can lead to the disappearance of the transition (section 2.1.1).
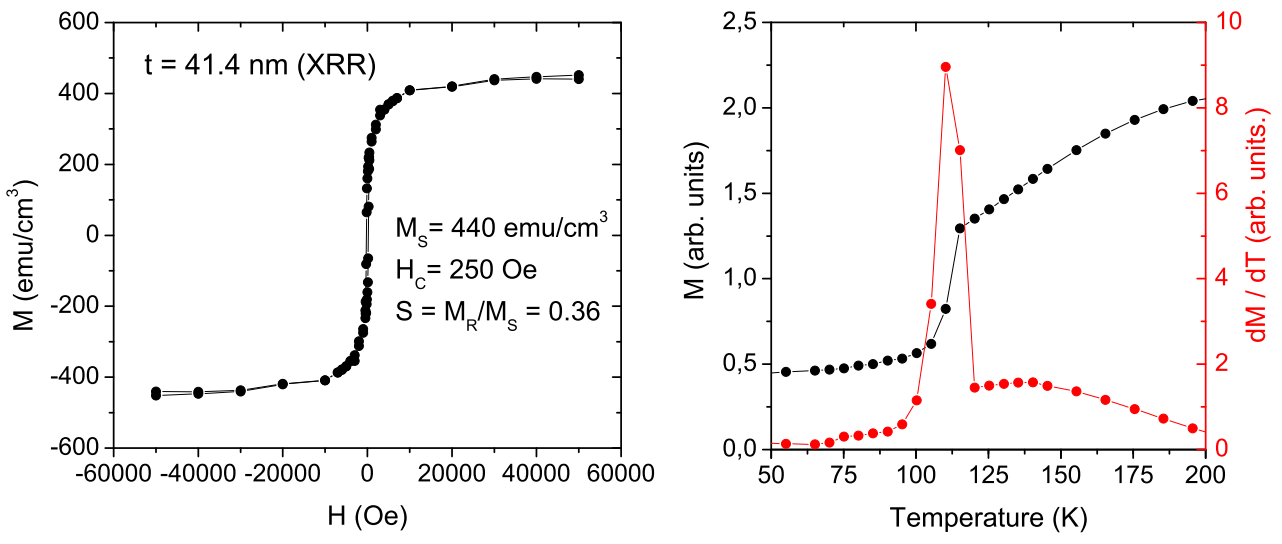

Figure 2.23: Magnetization measurement as a function of magnetic field at room temperature $(300 \mathrm{~K})$.
Figure 2.24: Magnetization as a function of the temperature in an applied field of 500 Oe. The sample is heated after a ZFC. 
As we have pointed out before, the Verwey transition can be also observed by means of transport measurements as a function of the temperature as a substantial increase of the resistivity takes place at the Verwey transition (figure 2.25). Therefore, we find $\mathrm{T}_{V}=111 \mathrm{~K}$, in agreement with the magnetization result obtained in the previous page.

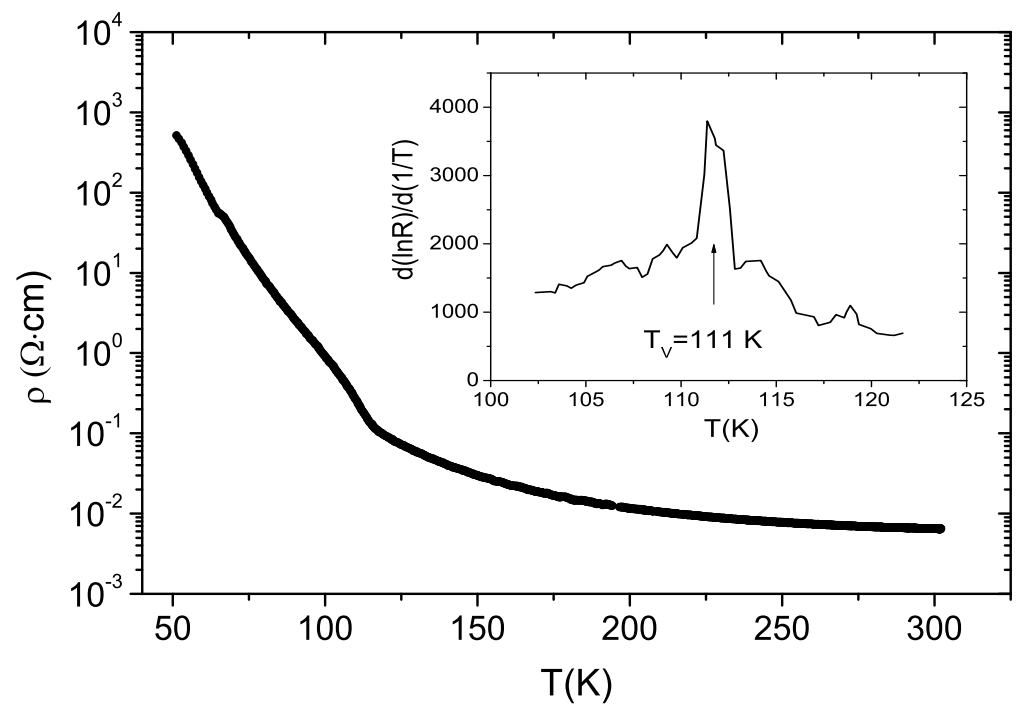

Figure 2.25: Resistivity measurement as a function of temperature. The Verwey transition temperature is found to be $111 \mathrm{~K}$.

On the other hand, the resistivity value at room temperature in our sample is $6.0 \mathrm{~m} \Omega \cdot \mathrm{cm}$, which is higher than the best values found in single-crystals $(\simeq 4 \mathrm{~m} \Omega \cdot \mathrm{cm})$. This behavior has been observed before by other authors, and has been explained as a consequence of the small film thickness where a higher APBs density can be found. Therefore, our resistivity value is higher than the bulk value, but low enough to reflect quite good crystallinity. 


\section{$2.3 \quad$ A thickness study}

In this section, an exhaustive systematic study is performed of the magnetic, structural and electrical transport properties as a function of the magnetite films thickness. With this purpose, samples with thickness ranging from $3 \mathrm{~nm}$ to $300 \mathrm{~nm}$ were deposited on $\mathrm{MgO}$ (001) substrates by means of PLD using a $\mathrm{KrF}$ excimer laser with $248 \mathrm{~nm}$ wavelength, $10 \mathrm{~Hz}$ repetition rate and $3 \times 10^{9}$ $W / \mathrm{cm}^{2}$ irradiance in an ultra-high-vacuum chamber. The optimal deposition parameters detailed in section 2.2 were used.

\subsubsection{Morphological and surface characterization}

The symmetrical $\theta-2 \theta$ scan around the (002) Bragg peak from the $\mathrm{MgO}$ substrate shows the $\mathrm{Fe}_{3} \mathrm{O}_{4}(004)$ reflection and its Laue oscillations (figure 2.26) of the films down to $9 \mathrm{~nm}$-thick, when the sample volume is too low and the diffraction peak too wide to provide an optimal diffraction peak over the background. Therefore, high structural coherence in the growth direction and low roughness is demonstrated.

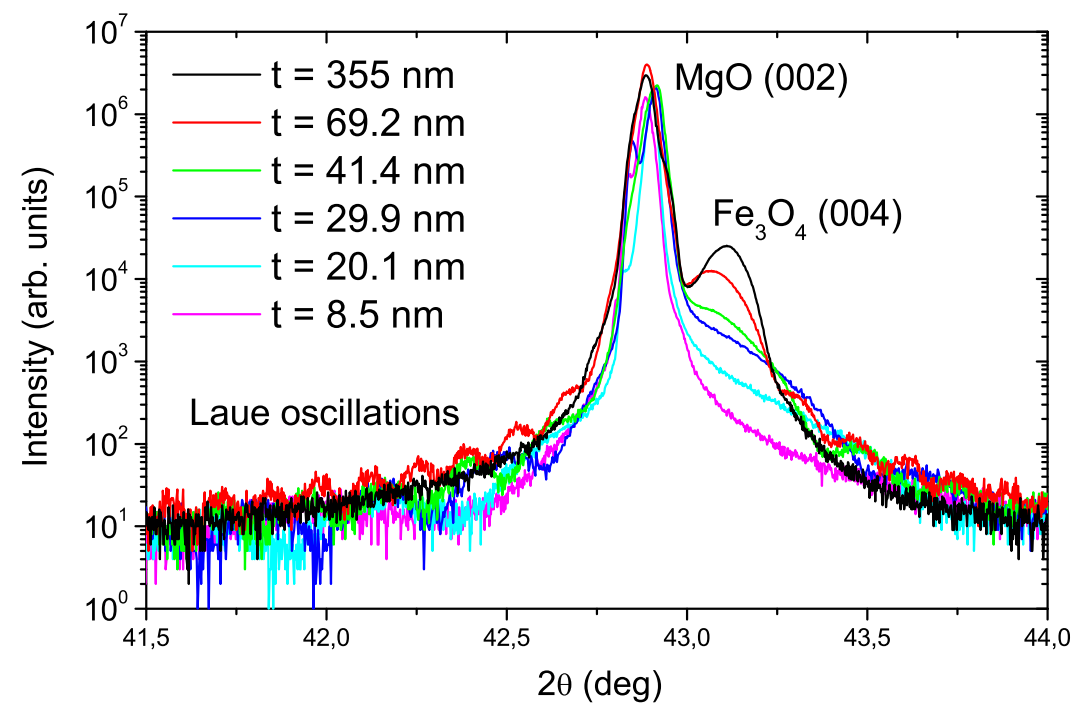

Figure 2.26: XRD measurements $(\theta-2 \theta)$ from $\mathrm{Fe}_{3} \mathrm{O}_{4}$ thin films with varying thickness.

Reciprocal Space Maps have been measured near the asymmetrical reflection (113) from the $\mathrm{MgO}$ and (226) from the $\mathrm{Fe}_{3} \mathrm{O}_{4}$ in order to study the epitaxial strain of our samples. 
As can be seen in figure 2.27 the samples remain completely strained for thicknesses under $150 \mathrm{~nm}$, as the magnetite in-plane lattice parameter is exactly the same as the $\mathrm{MgO}$ lattice parameter. The epitaxial stress starts to relax for the $300 \mathrm{~nm}$-thick film, being the $\mathrm{Fe}_{3} \mathrm{O}_{4}$ in-plane lattice parameter lower than the $\mathrm{MgO}$ lattice parameter.
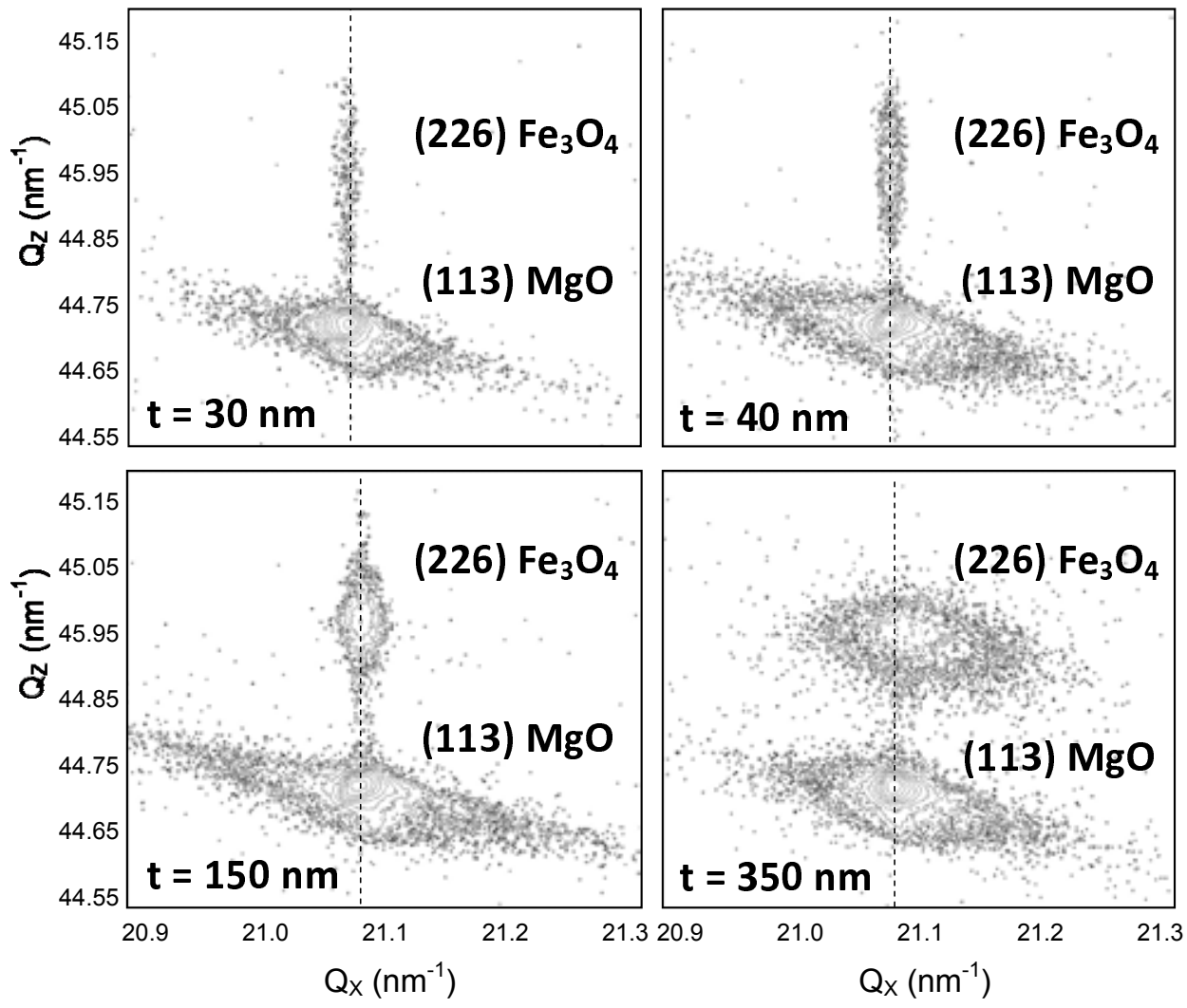

Figure 2.27: Reciprocal space maps in the vicinity of the asymmetrical (226) $\mathrm{Fe}_{3} \mathrm{O}_{4}$ and (113) $\mathrm{MgO}$ reflections for different magnetite film thicknesses. $\mathrm{Q}_{X}$ and $\mathrm{Q}_{X}$ are along the reciprocal space [110] and [001] directions, respectively.

XRR measurements from all the samples were performed in order to obtain the film thickness, the roughness $(\sigma)$ from the magnetite film and from the interface of the film with the substrate (table 2.3). The film roughness is similar to the substrate roughness value, revealing the smoothness of the magnetite layer, and the film density is close to the bulk value.

The surface study was performed by means of AFM (figure 2.28). It was observed that the magnetite films were continuous, independently to their thicknesses, showing a $2 \mathrm{D}$ growth and obtaining low RMS roughnesses of approximately $0.1 \mathrm{~nm}$. 
Therefore, all the grown films are atomically flat, which can be also seen in the surface profiles shown in figure 2.29.

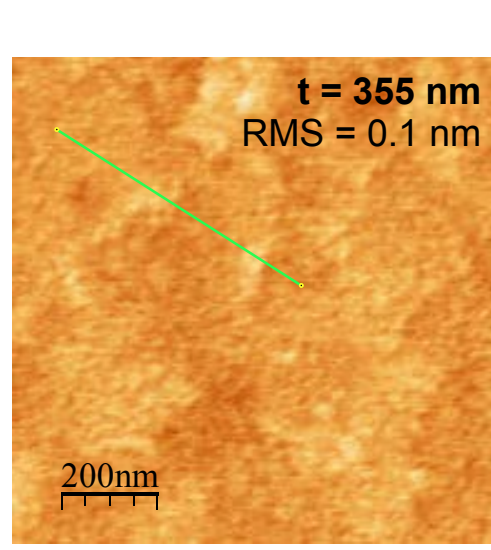

$10 \mathrm{~A}$

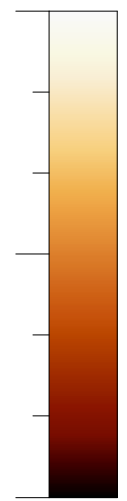

$0 \mathrm{~A}$

$1,5 \mathrm{~A}$
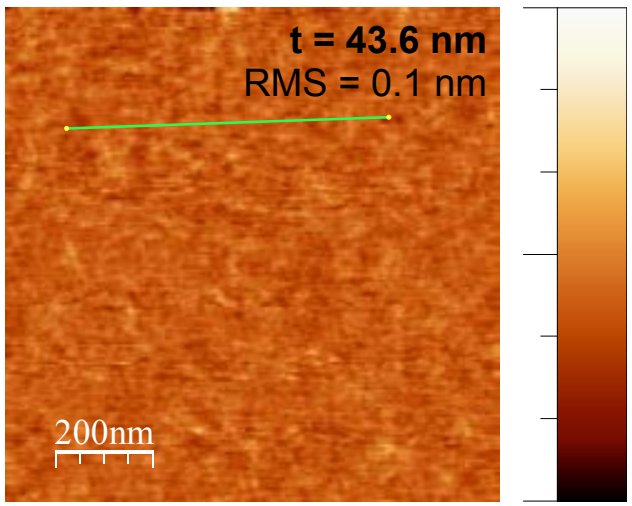

O A

$10 \mathrm{~A}$

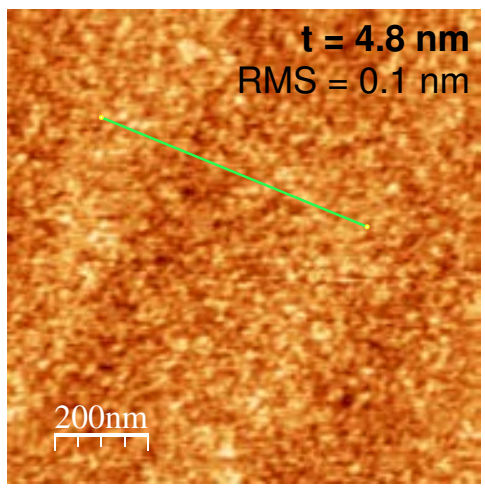

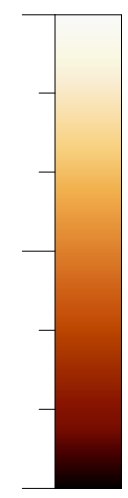

O A
$5 \mathrm{~A}$
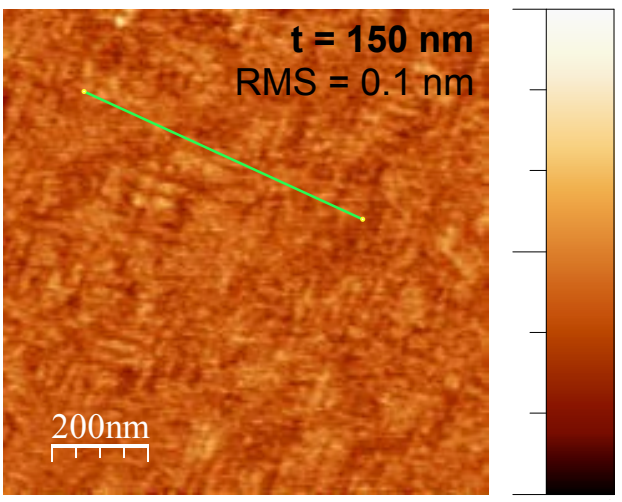

O A

$6 \mathrm{~A}$
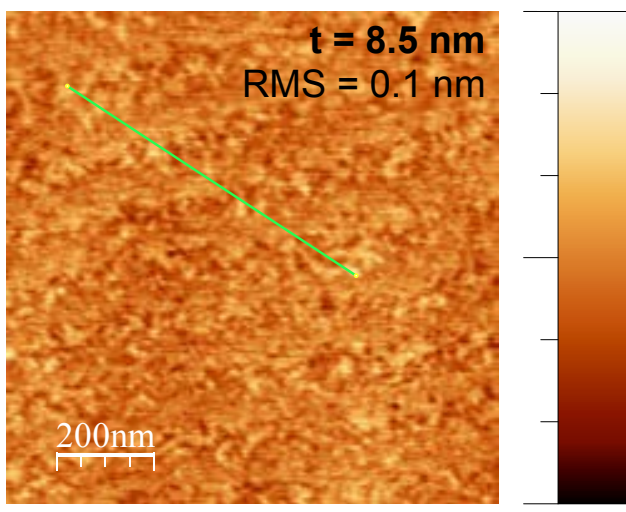

O A

Figure 2.28: $1 \mu m \times 1 \mu m$ AFM images from from $\mathrm{Fe}_{3} \mathrm{O}_{4}$ thin films of selected thicknesses in tapping mode with a 300 $\mathrm{kHz}$ tip. The lines define the profiles shown in image 2.29. 

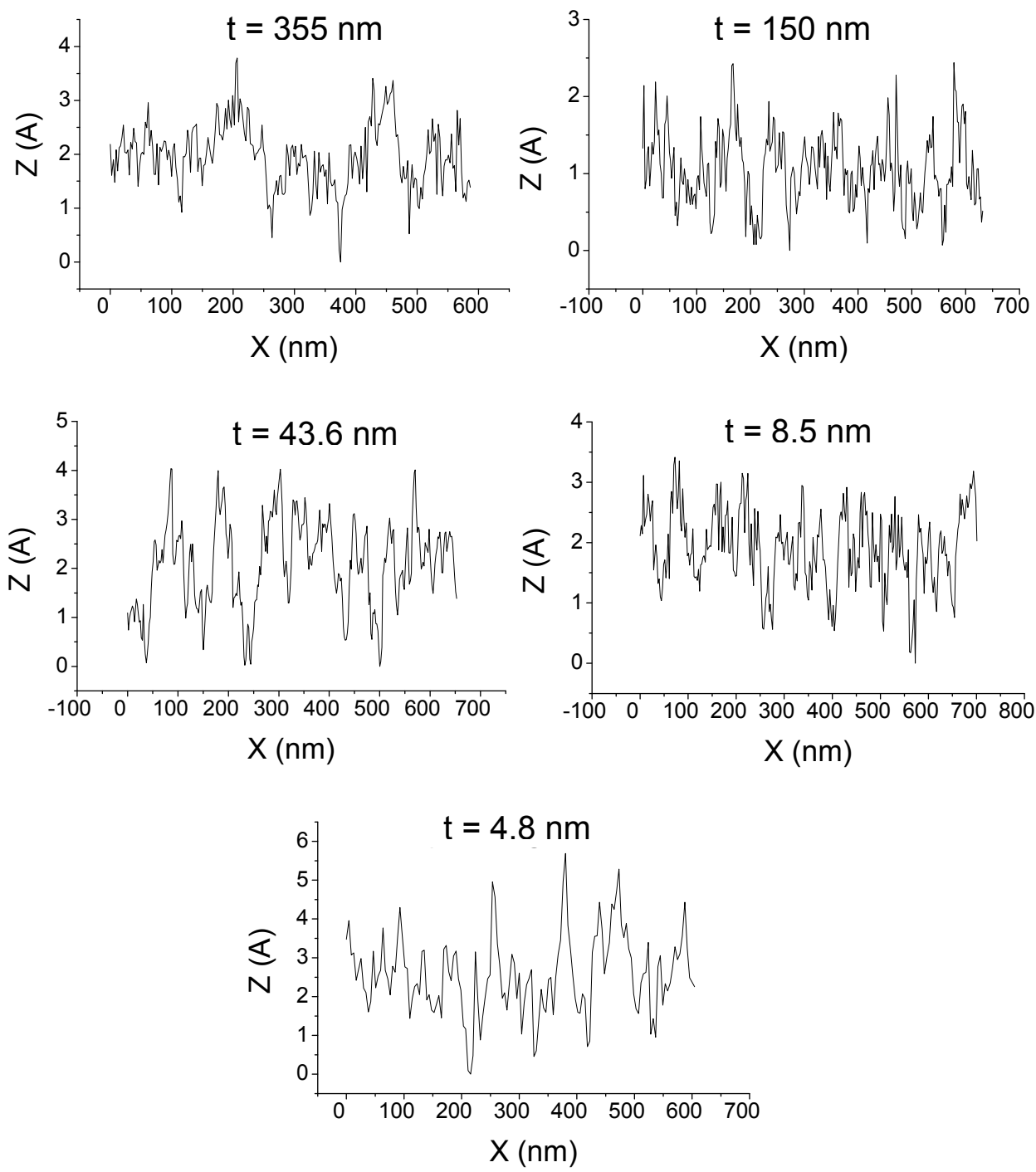

Figure 2.29: Surface profiles of magnetite thin films with different thicknesses.

\subsubsection{Magnetic behavior}

Magnetization measurements as a function of the temperature were performed by means of SQUID magnetometry in an applied magnetic field of 500 Oe in magnetite films with different thicknesses in order to monitor the Verwey transition. The Verwey transition presence down to $4 \mathrm{~nm}$ thick films (see figure 2.31) evidences the high quality of the samples, although a decrease and broadening of the transition temperature $\left(\mathrm{T}_{V}\right)$ for decreasing $\mathrm{Fe}_{3} \mathrm{O}_{4}$ thicknesses is observed. 
This behavior has been reported before in literature [124, 127, 128], and has been explained with different arguments, some of them pointed out in section 2.1.1).

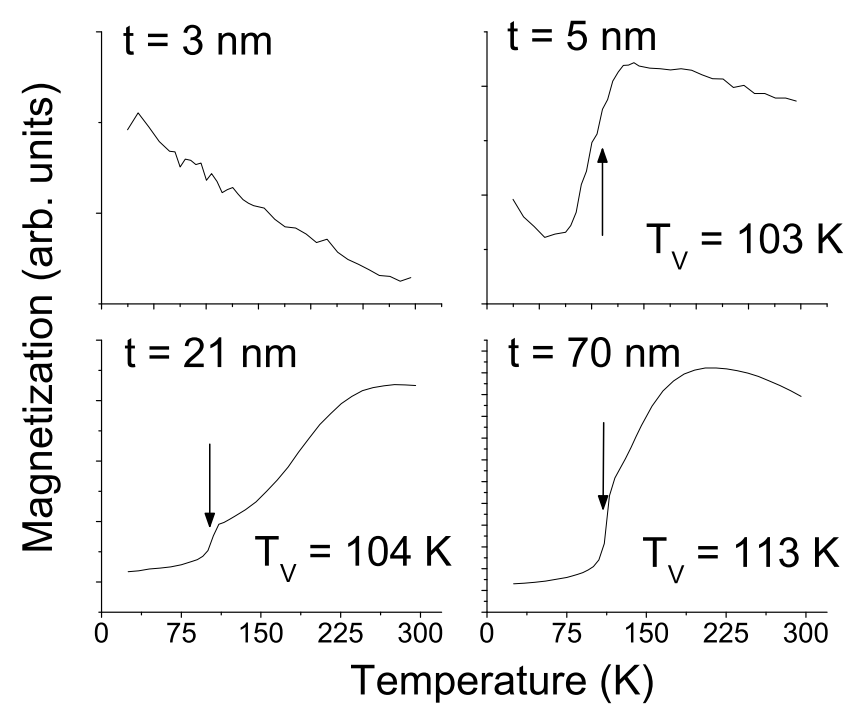

Figure 2.30: Magnetization as a function of the temperature in an applied field of 500 Oe of magnetite thin films with different thicknesses. The samples are heated after a ZFC.

It has been suggested that the decrease in $\mathrm{T}_{V}$ with film thicknesses could be due to the presence of defects such as APBs [119, 125]. In ultra thin films where the APB domain size is small, the long-range order of the $\mathrm{Fe}^{2+}$ and $\mathrm{Fe}^{3+}$ ions at the octahedral sites necessary for the Verwey transition to occur (section 2.1.1) can be inhibited, producing a decrease in $\mathrm{T}_{V}$ or even its disappearance in the case of the thinnest films, where the APB density is highest [125, 146]. Indeed, if we observe the Verwey transition temperature decrease as a function of $\mathrm{Fe}_{3} \mathrm{O}_{4}$ film thicknesses ( $\mathrm{t}$ ) (figure 2.31), it is easy to realize that the $\mathrm{T}_{V}$ dependence with $\mathrm{t}$ can be fitted to the expression given by equation 2.8, obtaining a thickness exponent:

$$
\begin{gathered}
T_{V}=T_{V}^{B U L K} \times\left(1-b \cdot t^{n}\right) \\
n=-0.43 \pm 0.14 \approx-0.5
\end{gathered}
$$

It has been reported that the APBs domain size (D) depends on the thickness of the magnetite films according to the relation $\mathrm{D} \approx \mathrm{t}^{1 / 2}$ (equation 2.3) [119, 133, 137]. Therefore, it can be concluded that $\mathrm{T}_{V}$ decreases with decreasing domain size, and thus with increasing APBs density, which suggests a correlation of the $\mathrm{T}_{V}$ behavior with the existence of APBs. 


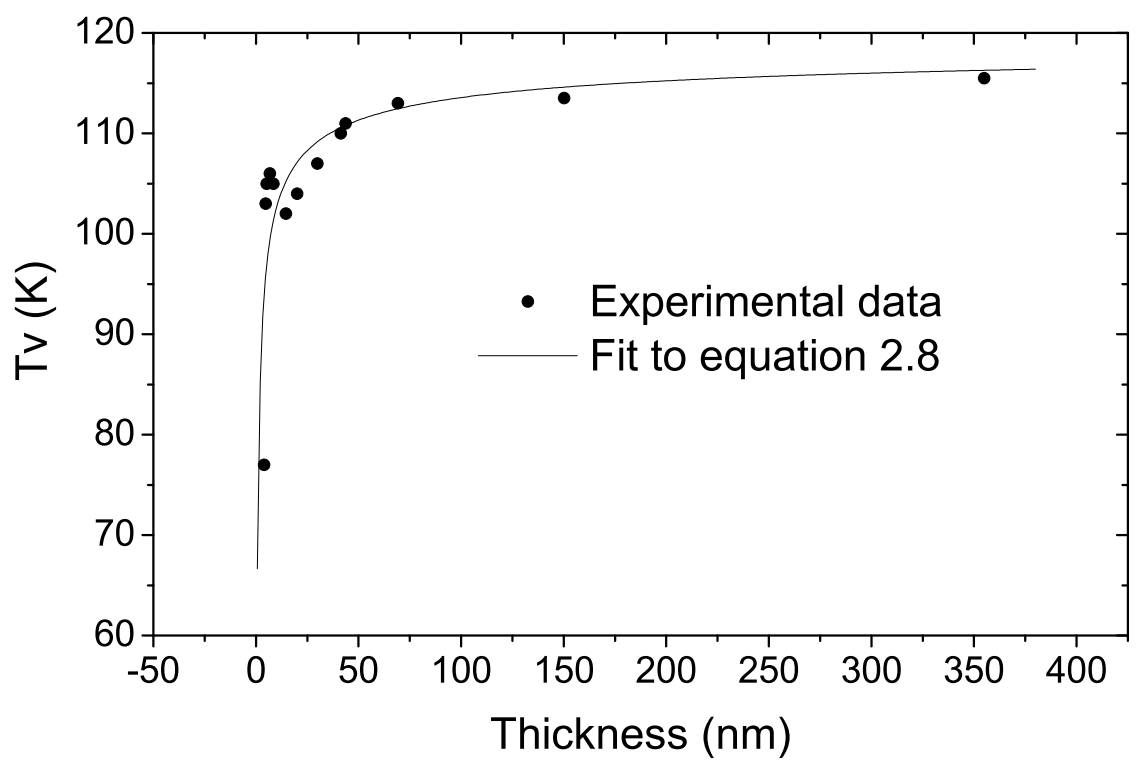

Figure 2.31: Verwey transition temperature as a function of $\mathrm{Fe}_{3} \mathrm{O}_{4}$ films thicknesses measured with a 500 Oe magnetic field, obtained from the maximum derivative of the magnetic moment as a function of the temperature. Samples were previously zero field cooled to $5 \mathrm{~K}$.

The magnetic hysteresis loops were measured at room temperature up to $5 \mathrm{~T}$ by means of SQUID magnetometry and applying the magnetic field in the film plane along the [100] direction at room temperature. Figure2.32 shows the dependence of the saturation magnetization with the film thickness, ranging from $2.8 \mathrm{~nm}$ to $300 \mathrm{~nm}$. The saturation magnetization values were determined by fitting the high-field data to a linear contribution in order to subtract the slope of the hysteresis loops due to the $\mathrm{MgO}$ substrate contribution.

For the films with thickness greater than $20 \mathrm{~nm}$, the obtained magnetization values are about $10-15 \%$ less than the value reported for bulk $\mathrm{Fe}_{3} \mathrm{O}_{4}(480$ $\mathrm{emu} / \mathrm{cm}^{3}$ or $4 \mu_{B} /$ f.u.), which coincides with literature [116, 119, 121, 129, 130, 142] and is generally explained as a consequence to the presence of APBs with antiferromagnetic interactions (see section 2.1.2).

The magnetite films with thicknesses below $20 \mathrm{~nm}$ exhibit increasing magnetization values as the thicknesses are reduced, up to values significantly larger than the bulk magnetic moment reported value. This anomalous magnetic behavior is widely studied in section 2.4 . 


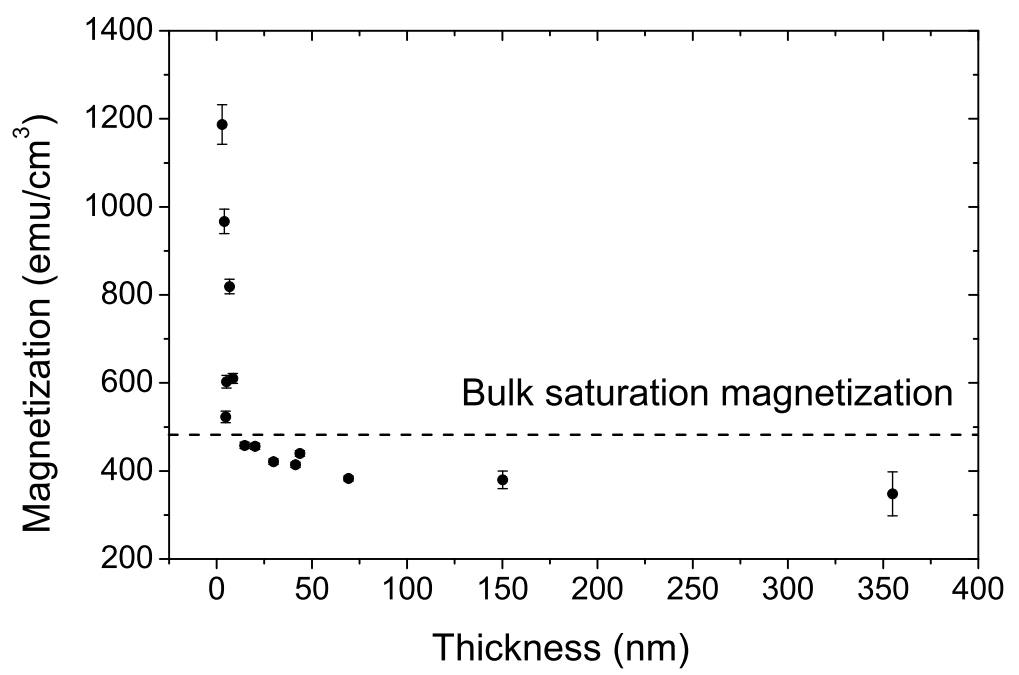

Figure 2.32: Magnetization measured at $300 \mathrm{~K}$ and a magnetic field of $5 \mathrm{~T}$ as a function of the film thickness.

The coercive fields $\left(\mathrm{H}_{C}\right)$ obtained from the hysteresis loops of magnetite films with different thickness are shown in figure 2.33. A decrease in $\mathrm{H}_{C}$ is observed for decreasing thickness and tends to zero for films below $4 \mathrm{~nm}$-thick, which indicates the appearance of a superparamagnetic behavior. This result is in agreement with results previously reported by other authors in literature [131, 132], and has been explained in an APB presence context (see section 2.1.2).

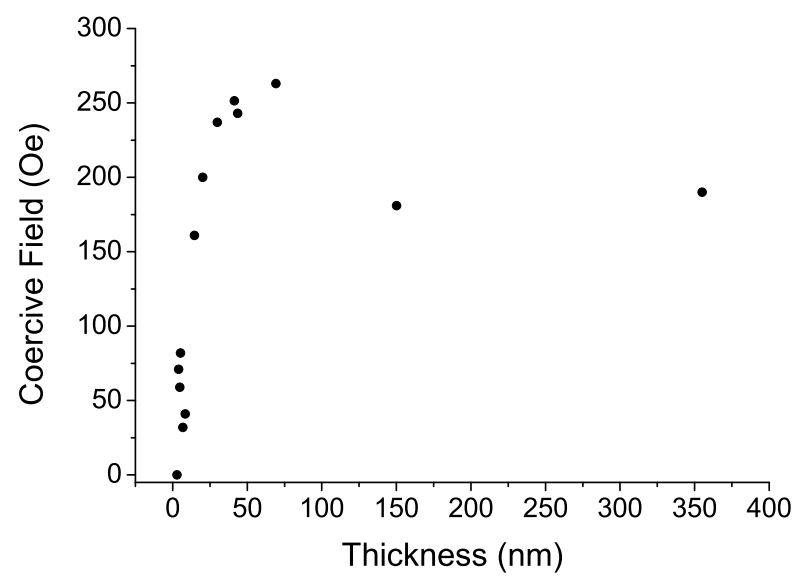

Figure 2.33: Coercive field measured at $300 \mathrm{~K}$ as a function of the film thickness. 


\begin{tabular}{||c||c|c|c||}
\hline \hline $\begin{array}{c}\text { Thickness } \\
(\mathrm{nm})\end{array}$ & $\begin{array}{c}\mathrm{T}_{V} \\
(\mathrm{~K})\end{array}$ & $\begin{array}{c}\mathrm{M}_{S} \\
\left(\mathrm{emu} / \mathrm{cm}^{3}\right)\end{array}$ & $\begin{array}{c}\mathrm{H}_{C} \\
(\mathrm{Oe})\end{array}$ \\
\hline \hline 355 & 115.5 & 348 & 190 \\
\hline 150.2 & 113.5 & 380 & 181 \\
\hline 69.2 & 113 & 383 & 263 \\
\hline 43.6 & 111 & 440 & 243 \\
\hline 41.4 & 110 & 414 & 251.5 \\
\hline 29.9 & 106.5 & 421 & 237 \\
\hline 20.1 & 104 & 456 & 200 \\
\hline 14.6 & 102 & 458 & 161 \\
\hline 6.8 & 114.5 & 819 & 32 \\
\hline 5.2 & 106 & 603 & 82 \\
\hline 4.8 & 103 & 523 & 59 \\
\hline 4.0 & 77 & 967 & 71 \\
\hline 2.8 & - & 1187 & 0 \\
\hline \hline
\end{tabular}

Table 2.3: Magnetic parameters for magnetite thin films with different thicknesses.

\subsubsection{Electrical transport}

The resistivity measurements were performed by means of the Van der Pauw method using a CCR to measure at different temperatures at the ICMA in Zaragoza (Spain) (see section 1.4).

Typical resistivity values ranging from $5,46 \mathrm{~m} \Omega \cdot \mathrm{cm}$ for the $150 \mathrm{~nm}$ film to $121,4 \mathrm{~m} \Omega \cdot \mathrm{cm}$ for the $5 \mathrm{~nm}$ film were obtained at room temperature, in agreement with the values obtained in other studies [119, 120, 133, 149]. The resistivity of the magnetite films is higher than the monocrystalline bulk material and indeed, it increases with decreasing film thicknesses. This behavior is related with the increase of APBs density in magnetite epitaxial thin films as their thicknesses decrease, due to their antiferromagnetic interaction, which leads to an increased resistivity [130, 134, 135] (see section 2.1.2).

The Verwey transition can be also observed in resistivity measurements as a function of the temperature (figure 2.34), and the values obtained are in agreement with the Verwey transition temperatures obtained from the magnetization measurements. The activation energy values $\left(\mathrm{E}_{a}\right)$ above and below $\mathrm{T}_{V}$ can both be obtained by fitting the resistivity values as a function of the temperature to the equation given for thermally activated hopping transport (equation 2.1 in section 2.1.1). The obtained values are displayed in table 2.4. together with results from other authors. 


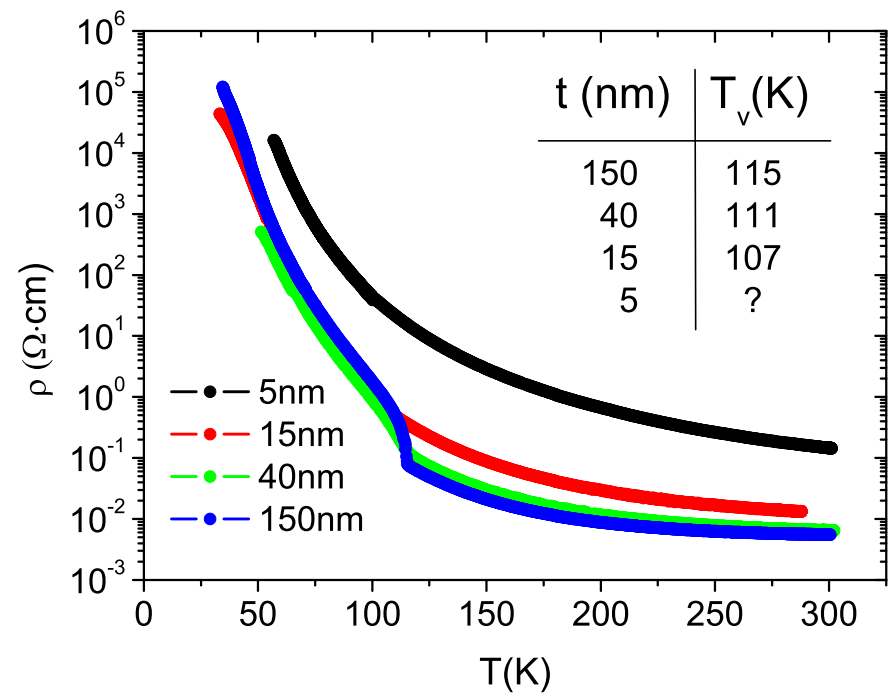

Figure 2.34: Resistivity as a function of the temperature for magnetite films with different thicknesses [75].

\begin{tabular}{||c||c||cc||}
\hline \hline & \multicolumn{1}{|c||}{$\begin{array}{c}\mathrm{E}_{a}(\mathrm{meV}) \\
(\mathrm{nm})\end{array}$} & $\mathrm{T}<\mathrm{T}_{V}$ & $\mathrm{~T}>\mathrm{T}_{V}$ \\
\hline \hline \multirow{3}{*}{ Experimental } & 150 & 56 & 53.5 \\
results & 40 & 65 & 50.1 \\
& 15 & 59 & 57 \\
& 5 & - & 70.6 \\
\hline \multirow{3}{*}{ Zhou et al [150] } & 4 & 68.9 & 67.8 \\
& 6 & 71.7 & 68.6 \\
& 8 & 67 & 64.1 \\
\hline \multirow{3}{*}{ Ziese et al [135] } & 200 & 69 & 56 \\
& 50 & 73 & 60 \\
& 15 & - & 59 \\
\hline Feng et al [101] & BULK & 105 & 60 \\
\hline \hline
\end{tabular}

Table 2.4: Activation energies determined above and below the Verwey transition for the samples studied and compared with the values obtained by other authors.

Whereas the high-temperature activation energies are in agreement with the bulk values reported by Zhou et al. [150], Ziese et al. [135] and Feng et al. [101], the low-temperature activation energies are slightly lower than literature values. 
Eerenstein et al. [133] used the effective medium approximation to describe the conductivity of the films grown by MBE as a function of the bulk and APB conductivities, $\sigma_{b}$ and $\sigma_{A P B}$ respectively, and of the fraction in which both phases are present $\left(\phi_{b}\right.$ and $\left.\phi_{A P B}\right)$, which follows the relation given by equation 2.6. Therefore, if we use the APB domain size (D) observed in a transmission electron microscopy plane image from a magnetite thin film with known thickness, the film conductivities as a function of sample thickness can be numerically fitted and $\sigma_{b}$ and $\sigma_{A P B}$ can be obtained

Direct visualization of the APB network in our samples has been obtained through TEM measurements in plane-view samples. The experiments were carried out by C. Magen in a Phylips CM30 LaB 6 microscope with a point resolution of $0.19 \mathrm{~nm}$ located at the CEMES-CNRS/Nanomat in Toulouse (France).

The inset of figure 2.35 shows the dark field image of a plane-view TEM specimen (40-nm-thick magnetite film). The experimental size distribution of the antiphase domains can be successfully fitted by a logarithmic-normal function as shown in Fig. 2.36. The mean D value obtained for this sample is $24.4 \mathrm{~nm}$ with a standard deviation of $13.9 \mathrm{~nm}$. This average domain size is comparable to other reported results for this film thickness [119].

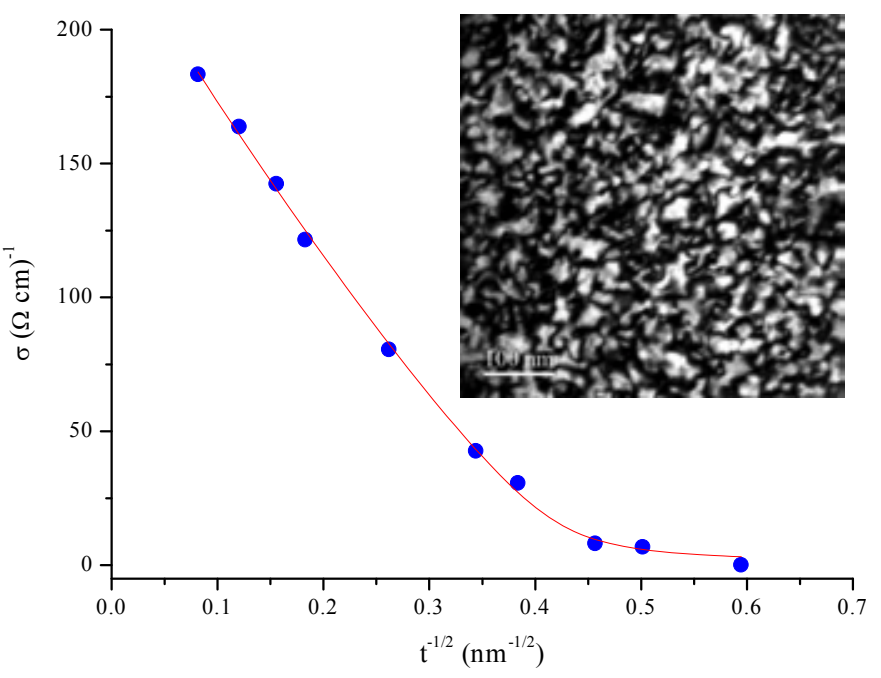

Figure 2.35: Experimental data (dots) of the value of conductivity as a function of $\mathrm{t}^{-0.5}$, where $\mathrm{t}$ is the film thickness and the corresponding fit (line) to the model developed in Ref. [133, 141]. The inset shows a TEM planar-view image of the 40nm-thick film where the APB network can be observed. The (220)-type reflection has been selected close to a two-beam condition, in which only the direct beam and one (220)-type reflection are strongly excited, near the [001] zone axis [151]. 


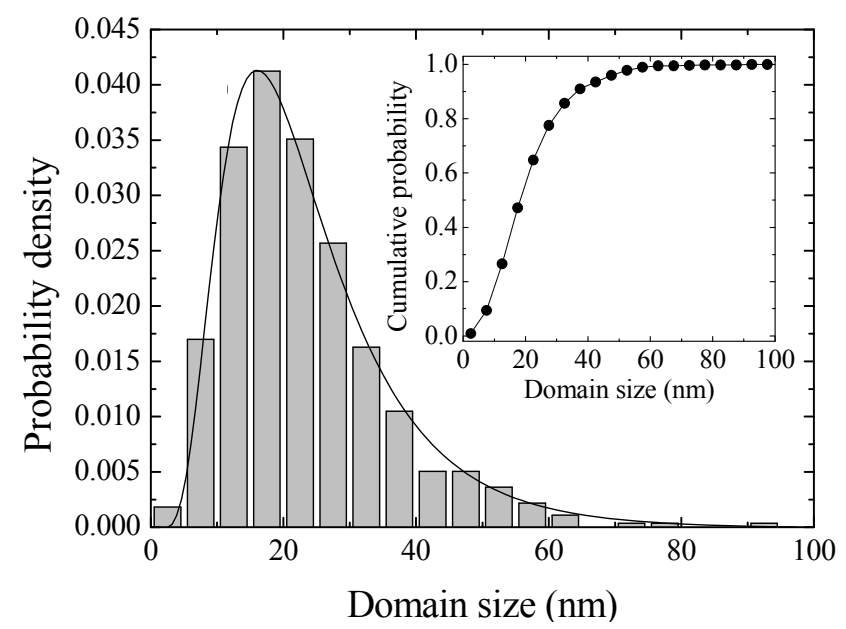

Figure 2.36: Size distribution of the antiphase domain size as calculated from the TEM image shown in figure 2.35 and the corresponding fit to a logarithmic-normal distribution. In the inset, the cumulative probability is shown as a function of the domain size 151].

From the fit to equations 2.6 and 2.5, the conductivities $(\sigma)$ and the boundary width $(\mathrm{d})$ can be extracted:

$$
\begin{gathered}
\sigma_{b}=(235 \pm 6) \Omega \cdot \mathrm{cm}^{-1} \\
\sigma_{A P B}=(1.0 \pm 3.9) \Omega \cdot \mathrm{cm}^{-1} \\
d=(2.6 \pm 0.3) \mathrm{nm}
\end{gathered}
$$

The value of $\sigma_{A P B}$ is negligible; indeed, it is expected to be zero in the case of perfect antiferromagnetic coupling at the boundaries.

The conductivity and APB thickness values are comparable with the ones obtained in magnetite thin films grown by MBE [133]:

$$
\begin{gathered}
\sigma_{b}=(260 \pm 12) \Omega \cdot \mathrm{cm}^{-1} \\
\sigma_{A P B}=(2 \pm 2.3) \Omega \cdot \mathrm{cm}^{-1} \\
d=(3.0 \pm 0.3) \mathrm{nm}
\end{gathered}
$$

Finally, we can assume that the results obtained from the systematic study of magnetite thin films as a function of the thickness are satisfactory, in good agreement with those published by other authors regarding magnetite films deposited by other methods and over different substrates. Therefore, it is guaranteed that our $\mathrm{Fe}_{3} \mathrm{O}_{4}$ films have quality enough to be used in spintronic devices. 


\subsubsection{Magnetotransport}

In this section we will give a general overview of the magnetotransport properties of epitaxial $\mathrm{Fe}_{3} \mathrm{O}_{4}$ thin films grown on $\mathrm{MgO}$ (001). A broad systematic characterization has been performed, the resistivity, magnetoresistance in different geometries, Hall effect and planar Hall effect for several film thicknesses and as a function of temperature being studied, obtaining interesting results that allowed the publication of several papers [151 154]. The magnetotransport measurements of the films were performed by A. Fernández-Pacheco, this being the subject of his $\mathrm{Ph}$. D. Thesis [75]. In this section we will summarize the main results obtained.

In general, in a magnetic material where a current density $(\vec{J})$ is flowing, an electric field $(\vec{E})$ proportional to $\vec{J}$ is established:

$$
\begin{aligned}
\vec{E} & =\widetilde{\rho} \vec{J} \\
\vec{E} & =\rho_{\perp}(B) \vec{J}+\left[\rho_{\|}(B)-\rho_{\perp}(B)\right](\vec{m} \cdot \vec{J}) \vec{m}+\rho_{H}(B) \vec{m} \times \vec{J}
\end{aligned}
$$

where $\widetilde{\rho}$ is the resistivity tensor and $\vec{m}$ is the normalized magnetization $(\mathrm{m}=$ $\mathrm{M} / \mathrm{M}_{0}$ ). The second term accounts for the AMR and the Planar Hall Effect (PHE), and the third term corresponds to the anomalous Hall Effect (AHE).

\section{Planar Hall Effect}

The planar Hall Effect in magnetic materials occurs due to the difference in resistivity when the current and the magnetization are parallel $\left(\rho_{\|}\right)$or perpendicular $\left(\rho_{\perp}\right)$ to each other, the physical origin being the same as the AMR [155.

In the measurement geometry of the PHE, both the magnetic field and the current are applied in the film plane. In a simple but common situation with a single-domain sample showing in-plane magnetization $(\vec{M})$, when a current density $\vec{J}$ is applied along the x direction and $\vec{M}$ forms an angle $\theta$ with the $\vec{J}$ direction, an electric field appears given by

$$
\begin{array}{lrl}
E_{x}=J \rho_{\perp}+J\left(\rho_{\|}-\rho_{\perp}\right) \cos ^{2} \theta & \rho_{x x}=\frac{E_{x}}{J} \\
E_{y}=J\left(\rho_{\|}-\rho_{\perp}\right) \sin \theta \cos \theta & \rho_{x y}=\frac{E_{y}}{J}
\end{array}
$$

While the longitudinal component of this electrical field, $\mathrm{E}_{x}$, gives rise to the AMR effect with resistivity $\rho_{x x}$, its transversal component, $\mathrm{E}_{y}$, gives rise to the PHE effect with $\rho_{x y}$. 
Due to the relation between the magnetization direction and the PHE, the PHE has been used in thin films as a sensitive tool to study in-plane magnetization processes when transport measurements are more suitable than direct measurements of the magnetization [156, 157]. The PHE can be also used for sensing low magnetic fields, showing advantages with respect to AMR sensors in terms of thermal drift [158, 159].

Recently, the discovery of the so-called giant planar Hall effect (GPHE) found in the ferromagnetic semiconductor $\left(\mathrm{Ga}_{1-x} \mathrm{Mn}_{x}\right)$ As [160] has attracted much interest. The effect at liquid helium temperature for this compound is four orders of magnitude higher than the existing in ferromagnetic metals, reaching values for $\rho_{x y}$ of a few $m \Omega \cdot \mathrm{cm}$. The discovery has resulted in a thorough study of the magnetization properties of the material by means of this effect 161 164]. The system $\mathrm{Ga}_{1-x} \mathrm{Mn}_{x}$, with $\mathrm{T}_{C}$ below $160 \mathrm{~K}$, is restricted for applications of GPHE in magnetic sensing at low temperatures. In the search for giant PHE at room temperature, promising results have been found in $\mathrm{Fe}_{3} \mathrm{O}_{4}$ at room temperature [165, 166]. Therefore, we have investigated the giant $\mathrm{PHE}$ in epitaxial thin films of $\mathrm{Fe}_{3} \mathrm{O}_{4}$ grown on $\mathrm{MgO}$ (001) substrates in a wide range of thicknesses and temperatures.

The PHE was studied at room temperature in magnetite films with thicknesses 5, 9, 15, 40 and $150 \mathrm{~nm}$, as a function of the applied magnetic field (Fig. 2.37). The measurements were done with the applied magnetic field forming a fixed angle with the current, $\theta=45^{\circ}$, since the signal will be maximum in this geometry when saturation in magnetization is reached (Equation 2.12):

$$
E_{y}^{\left(45^{\mathrm{o}}\right)}=\rho_{x y}^{\left(45^{\mathrm{o}}\right)} J=\frac{\rho_{\|}-\rho_{\perp}}{2} J
$$

The values for $\rho_{x y}$ at maximum magnetic field are of the same order as those reported by other authors [165, 166], and a continuous decrease in magnitude is observed with increasing film thickness. A record value at room temperature of $\left|\rho_{x y}\right| \approx 60 \mu \Omega \cdot \mathrm{cm}$ is obtained for the 5-nm-thick film.

The peaks associated with the coercive field shift towards zero field as the sample thickness is decreased, indicating a evolution of the films towards superparamagnetic behavior, also observed in the magnetization measurements showed previously in section 2.3 .2 .

In order to study the evolution of the GPHE as a function of temperature, 20-nm-thick and 40-nm-thick samples were selected and patterned in a twostep lithography process to define a Hall geometry (width $=300 \mu m$ ) which minimizes transversal voltage offsets, really appreciable below the Verwey transition [153]. 


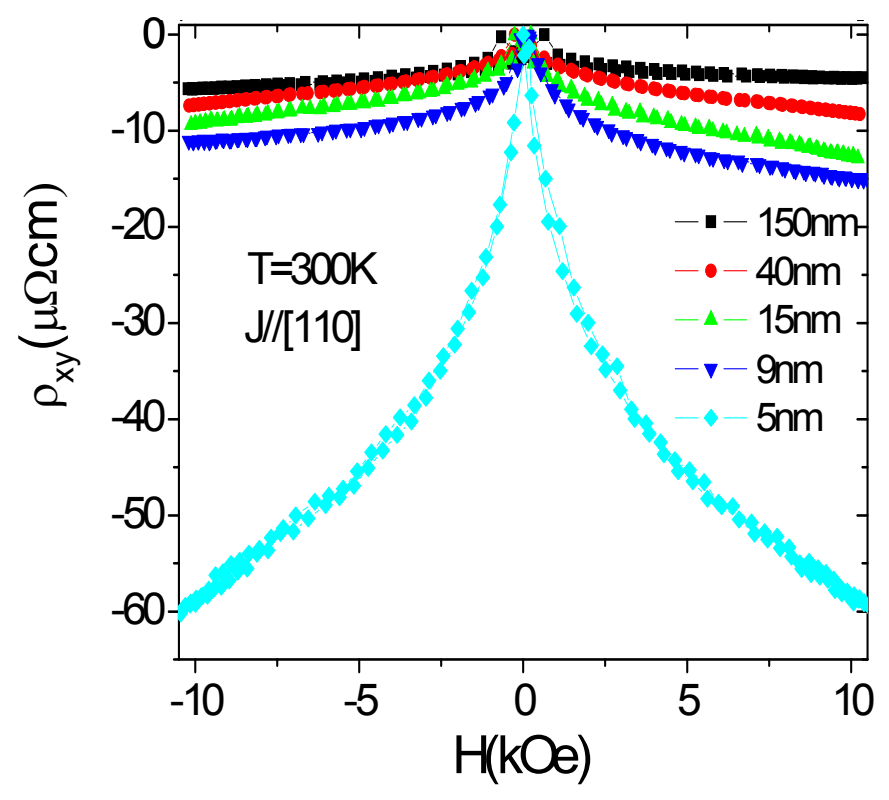

Figure 2.37: Transversal resistivity as a function of the applied magnetic field $\theta=45^{\circ}$ for several thin-film thicknesses at room temperature with current direction $\mathrm{J} / /[110]$ [75, 151].

As a function of temperature the planar Hall effect increases in magnitude, reaching colossal values below the Verwey transition, $16 \mathrm{~m} \Omega \cdot \mathrm{cm}$ at $\mathrm{T}=73 \mathrm{~K}$ for the 20-nm-thick film. This result demonstrates the potentiality of the planar Hall effect in magnetite thin films for studies of magnetization processes as well as for sensitive low-field detection.

\section{Anomalous Hall Effect}

In magnetic materials, when a magnetic field is applied perpendicularly to the film plane and a current is injected in the $\mathrm{x}$ direction, in addition to the transversal voltage proportional to the applied magnetic field that corresponds to the Ordinary Hall Effect (OHE), it appears a term derived from the spontaneous magnetization of the material. This additional contribution is the Anomalous Hall Effect (AHE), accounted in the last term in equation 2.10.

Therefore, the Hall resistivity can be written as a sum of the OHE proportional to the applied magnetic field and the AHE proportional to the magnetization of the material, which is in general larger than the first term in ferromagnets:

$$
\rho_{H}=\rho_{x y}=\mu_{o}\left(R_{0} H+R_{A} M\right)
$$


The origin of the AHE has been a controversial topic in the last years. However, the most recent theory by S. Onoda et al. [167] seems to have shed some light to it, distinguishing three regimes as a function of the longitudinal conductivity: a clean regime with extremely high conductivity $\left(\sigma_{x x}>\right.$ $\left.10^{6} \Omega^{-1} \mathrm{~cm}^{-1}\right)$, an extrinsic-to-intrinsic crossover at lower conductivities $\left(10^{4}\right.$ $\left.\Omega^{-1} \mathrm{~cm}^{-1}<\sigma_{x x}<10^{6} \Omega^{-1} \mathrm{~cm}^{-1}\right)$ and a dirty regime $\left(\sigma_{x x}<10^{4} \Omega^{-1} \mathrm{~cm}^{-1}\right)$.

We have studied systematically the AHE in epitaxial thin films of magnetite grown on $\mathrm{MgO}$ (001) substrates as a function of film thickness (from 5 to 150 $\mathrm{nm})$ and temperature $(60 \mathrm{~K}<\mathrm{T}<300 \mathrm{~K})$ [75, 153].

The Hall resistivity was found to be negative for all the measured temperature range, presenting a huge enhancement at temperatures below the Verwey transition, reaching values above $1 \mathrm{~m} \Omega \cdot \mathrm{cm}$. The anomalous Hall conductivity scales as $\sigma_{H} \approx \sigma_{x x}^{1.6}$ (dirty regime of conduction) over four decades of the longitudinal conductivity $\sigma_{x x}$ in all the samples, irrespective of thickness or temperature. Comparison with literature confirms this behavior is general for this compound [167], indicating the universality of this relationship in the dirty regime of conductivities (Figure 2.38).

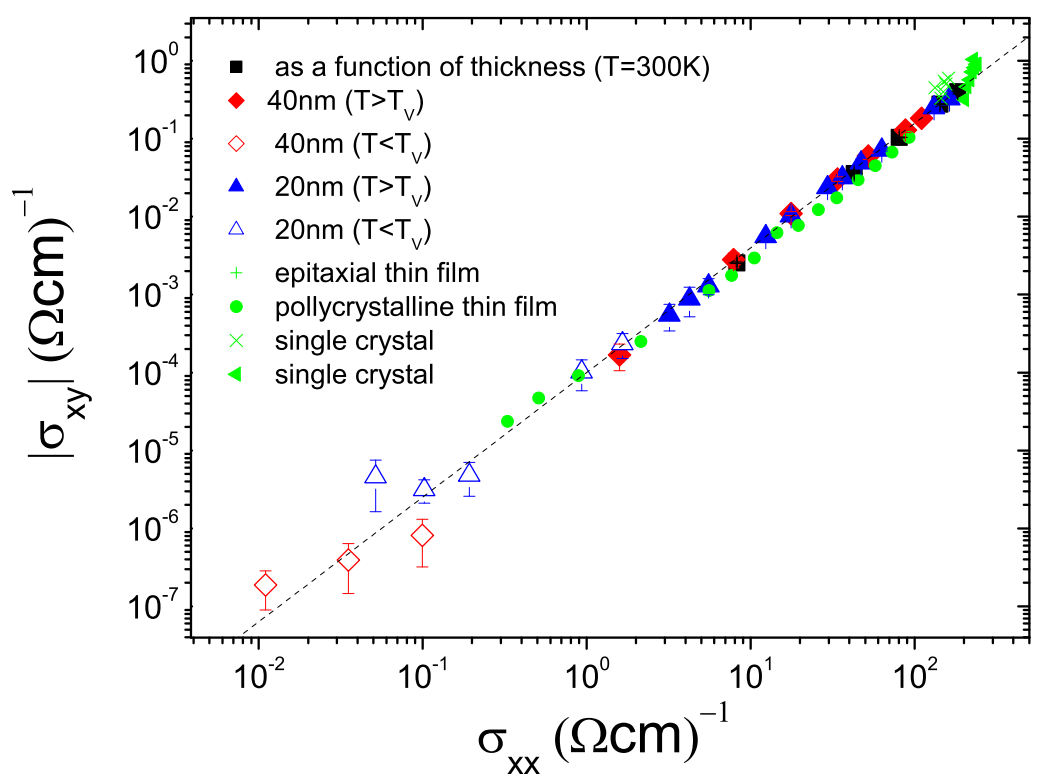

Figure 2.38: Magnitude of the Hall conductivity at $11 \mathrm{kOe}$ as a function of the longitudinal conductivity for our films [75, 153] and data taken from references [149, $168-170$. 


\section{Ordinary Hall Effect}

The ordinary Hall constant $\left(\mathrm{R}_{0}\right)$ can be obtained from the high-field slope of the Hall effect, as once the sample reaches its magnetic saturation the contribution of the AHE is constant. Therefore:

$$
R_{0}=\frac{d \rho_{H}}{d\left(\mu_{0} H\right)}
$$

And from $R_{0}$ the density of conducting carriers can be calculated, considering the conduction dominated by electrons:

$$
N=-\frac{1}{R_{0} e}
$$

However, it is well known that magnetite films are difficult to saturate in moderate magnetic fields, as the APBs separate oppositely magnetized regions (see section 2.1.2), and as a consequence, a clear determination of the $\mathrm{OHE}$ is not possible from our previous measurements.

We have measured the Hall effect and the magnetoresistance of epitaxial $\mathrm{Fe}_{3} \mathrm{O}_{4}$ thin films with thicknesses of 200, 41, 27 and $16 \mathrm{~nm}$ grown on $\mathrm{MgO}$ (001) in magnetic fields up to $30 \mathrm{~T}$ in the very high static magnetic fields at the High Field Magnet Laboratory (HFML) in Nijmegen (The Netherlands) (see figure 2.39). Using such high fields, it is possible to magnetically saturate films thicker than $40 \mathrm{~nm}$, providing the possibility to separate the OHE from the anomalous one.

The electron density calculated for the 41nm-thick-film $\left(0.93 e^{-} / f . u\right.$. $)$ contradicts the values published by some authors [149, 171]. However, their measurements were performed up to $7 \mathrm{~T}$ and therefore, it can be assumed that their magnetite samples are not saturated, obtaining a higher slope, thus a smaller value for $n$.

We find an effective electron density corresponding to 1 electron per f.u, in agreement with the valued reported by other authors [168, 172] in magnetite polycrystalline thin films and in a single crystal, and also agrees with the Verwey conduction model at high temperature, which predicted $\mathrm{n}=1$ $e^{-} / f . u$. [79]. A smaller value is obtained for thinner films, caused by the increasing density of APBs defects, which produces a smaller effective density of conducting electrons. 


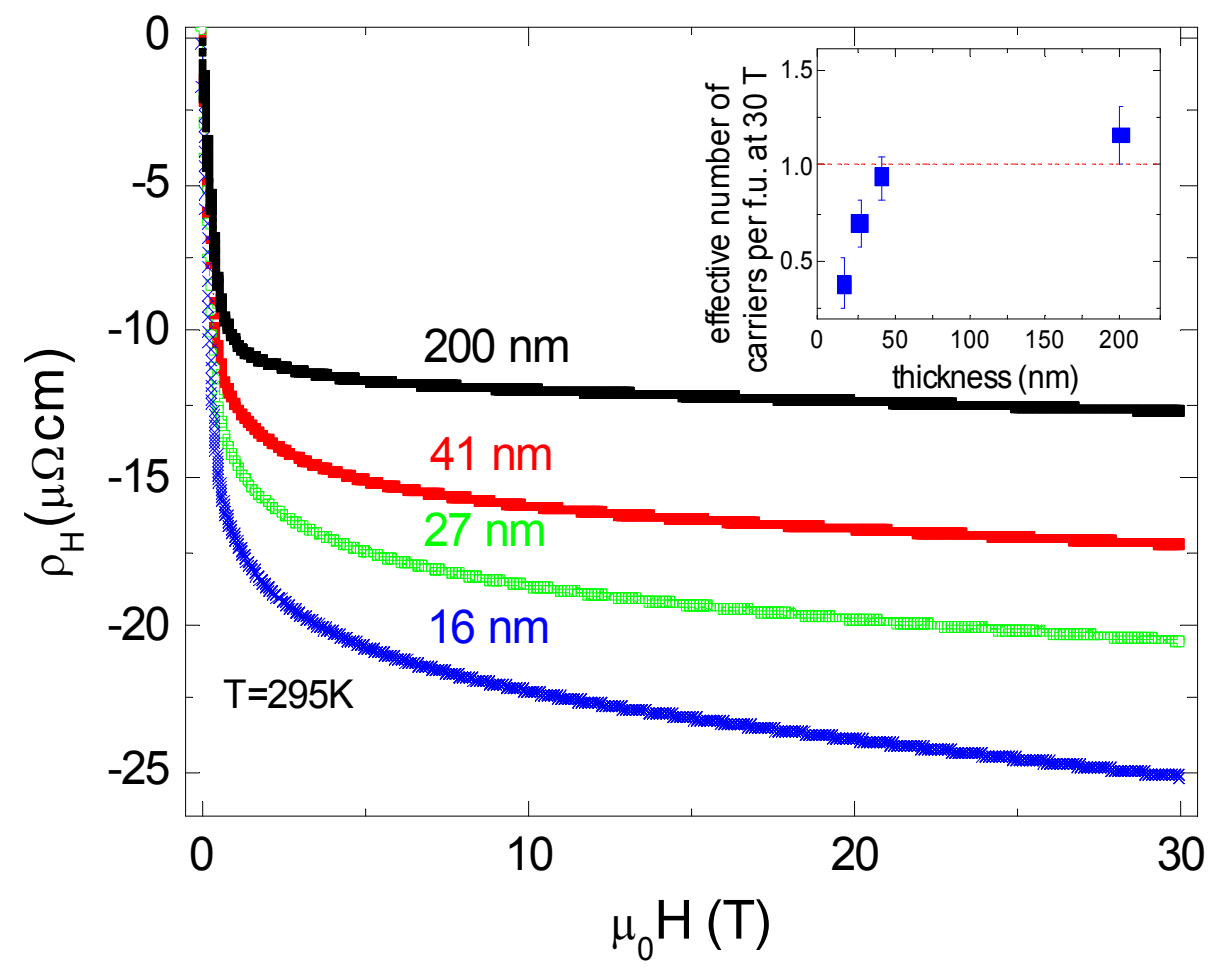

Figure 2.39: Hall resistivity up to $30 \mathrm{~T}$ at RT for four samples, varying in thickness. The inset shows the effective density of electrons at the maximum field. The value associated to 1 e- per f.u. is marked by a dashed line [75, 154]. 


\subsection{Enhanced magnetic moment in epitaxial $\mathrm{Fe}_{3} \mathrm{O}_{4}$ ultra-thin films}

In section 2.3, it was found that magnetite films grown on $\mathrm{MgO}$ (001) with thicknesses below $20 \mathrm{~nm}$ exhibit increasing magnetization values as the thicknesses are reduced, up to values significantly larger than the bulk magnetic moment reported value.

Recent studies performed by Arora et al. [9, 10] on well characterized epitaxial $\mathrm{Fe}_{3} \mathrm{O}_{4}$ thin films grown on $\mathrm{MgO}$ (100) by MBE show that the ultrathin films ( $<5 \mathrm{~nm}$ thickness) are ferromagnetic and their magnetization is much greater than that of bulk magnetite. The main factor contributing to the observed enhanced magnetic moment is proposed to be the noncompensation of spin moments between the tetrahedral and octahedral sublattices at the surface, at the interface with the substrate, and at the antiphase-domain boundaries. They suggest a model in which the magnetite films consist of a layer with bulk properties (bulk saturation magnetization $\mathrm{M}_{S}$ ) plus surface contribution of moment $\mathrm{M}_{i}$ at the $\mathrm{Fe}_{3} \mathrm{O}_{4} / \mathrm{MgO}$ and $\mathrm{Fe}_{3} \mathrm{O}_{4} /$ air interfaces, resulting in a magnetization given by:

$$
M=M_{S}+\frac{M_{i}}{t}
$$

were $t$ is the film thickness.

Similar results have been found for the spinel ferrite $\mathrm{NiFe}_{2} \mathrm{O}_{4}$ grown on $\mathrm{SrTiO}_{3}$ substrates [173], showing for $3 \mathrm{~nm}$-thick films a magnetization enhancement of almost four times the bulk value, as measured by means of SQUID magnetometry. This effect was ascribed to the cation inversion: the presence of $\mathrm{Ni}^{2+}$ in $\mathrm{A}$ sites and $\mathrm{Fe}^{3+}$ in $\mathrm{B}$ sites would increase the magnetic moment. However, recent x-ray Magnetic Circular Dichroism (XMCD) and resonant photoemission experiments have ruled out this hypothesis [174].

We have obtained similar magnetization results to those reported by Arora [9, 10, 175]. In section 2.3, the magnetic hysteresis loops of magnetite films with thicknesses ranging from 2.8 to $350 \mathrm{~nm}$ were measured at room temperature up to $5 \mathrm{~T}$ using by means of SQUID magnetometry and applying the magnetic field in the film plane along the [100] direction. The saturation magnetization values were determined by fitting the high-field data to a linear dependence in order to subtract the slope of the hysteresis loops due to the $\mathrm{MgO}$ substrate contribution.

We found that the magnetite films with thicknesses below $20 \mathrm{~nm}$ exhibit increasing magnetization values as the thicknesses are reduced, up to values significantly larger than the bulk magnetic moment reported value. 
By fitting the saturation magnetization values shown in figure 2.40 to the expression given by Arora (equation 2.17), we obtained a bulk saturation magnetization $\mathrm{M}_{S}=349 \pm 41 \mathrm{emu} / \mathrm{cm}^{3}$ and a surface contribution $\mathrm{M}_{i}=2088 \pm$ $274 \mathrm{emu} / \mathrm{cm}^{3}$ in agreement with Refs. [9, 10].

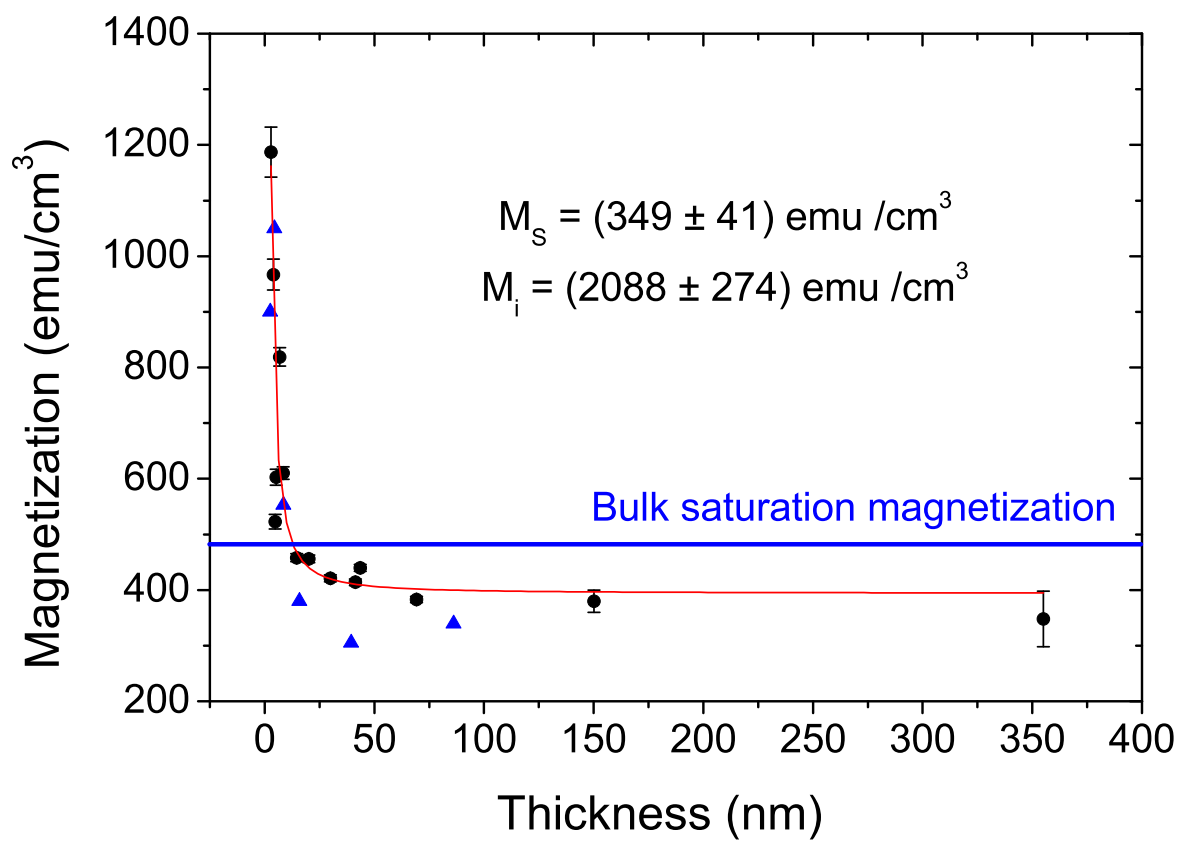

Figure 2.40: Saturation magnetization measured at $300 \mathrm{~K}$ as a function of the film thickness at $5 \mathrm{~T}$ for the standard magnetite films (black dots) and at $1.9 \mathrm{~T}$ for samples grown specifically for PNR measurements (blue triangles). The solid red line represents a fit to the data using equation 2.17

At this point, an investigation needs to be performed in order to identify the origin of the anomalous giant magnetic moment in epitaxial $\mathrm{Fe}_{3} \mathrm{O}_{4}$ thin films grown on $\mathrm{MgO}$ (001). With this purpose, the magnetic characterization of our magnetite layers was completed with PNR and XMCD experiments, and the chemical analysis of the films was investigated by x-ray Photoemission Spectroscopy (XPS). The $\mathrm{MgO}(001)$ substrates were also characterized by SQUID magnetometry and Inductively Coupled Plasma Mass Spectroscopy (ICP). 


\subsubsection{Polarized Neutrons Reflectivity characterization}

PNR measurements were performed in order to understand the anomalous magnetic behavior of the magnetite ultra-thin films, as the PNR is a specially suited technique to the measurement of magnetization and magnetic depth profile in thin films. The reflectivity profiles are different depending on the layer magnetization value, thickness or roughness (from a magnetic point of view). Therefore, it may determine whether the enhanced magnetic moment is confined at the surface of the film, at the interface with the substrate, or is homogeneously distributed over the film thickness, as can be seen in figure 2.41, where different magnetic configurations are simulated for a $4.5 \mathrm{~nm}$ - thick magnetite film by means of Parrat specific software, which uses the Parrat algorithm for reflectivity [176].
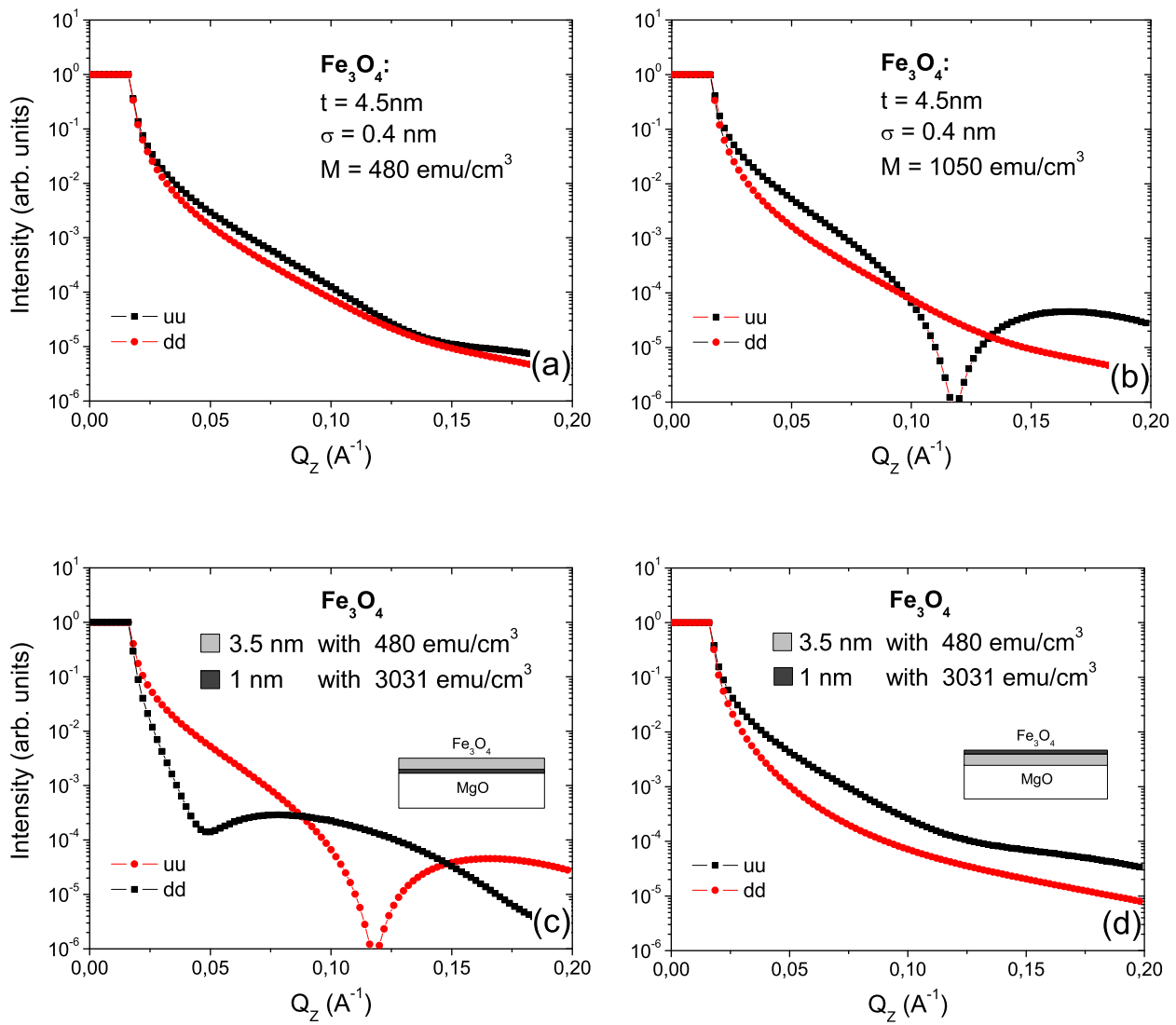

Figure 2.41: PNR simulations from a $4.5 \mathrm{~nm}$-thick $\mathrm{Fe}_{3} \mathrm{O}_{4}$ film with bulk magnetic moment (a), enhanced moment homogeneously distributed (b), and enhanced moment localized in $1 \mathrm{~nm}$ layer, either at the interface with the substrate (c) or at the film surface (d). The simulations have been performed with Parrat software. 
Magnetite films with different thicknesses were grown on $\mathrm{MgO}(001)(1 / 2$ " × $1 / 2$ " $\times 0.5 \mathrm{~mm}$ ) specifically for PNR measurements in order to improve the magnetic signal with bigger samples. The samples were structurally characterized by XRD showing high crystallinity. VSM measurements were performed with the magnetic field applied in the film plane, up to a maximum field of 1.9 Tesla (see figure 2.40), obtaining and a magnetic behavior similar to the standard magnetite films grown on $\mathrm{MgO}(001)(5 \mathrm{~mm} \times 5 \mathrm{~mm} \times 0.5 \mathrm{~mm})$, previously measured by SQUID.

PNR measurements were performed at the Institut Laue-Langevin (ILL) on the D17 vertical sample plane reflectometer, at room temperature with an applied in-plane magnetic field of $1 \mathrm{~T}$ (Figure 2.42). Since the applied magnetic field was large enough to mostly saturate the sample, all of the magnetization is essentially parallel to the applied field, thus, the spin-flip scattering is negligible.
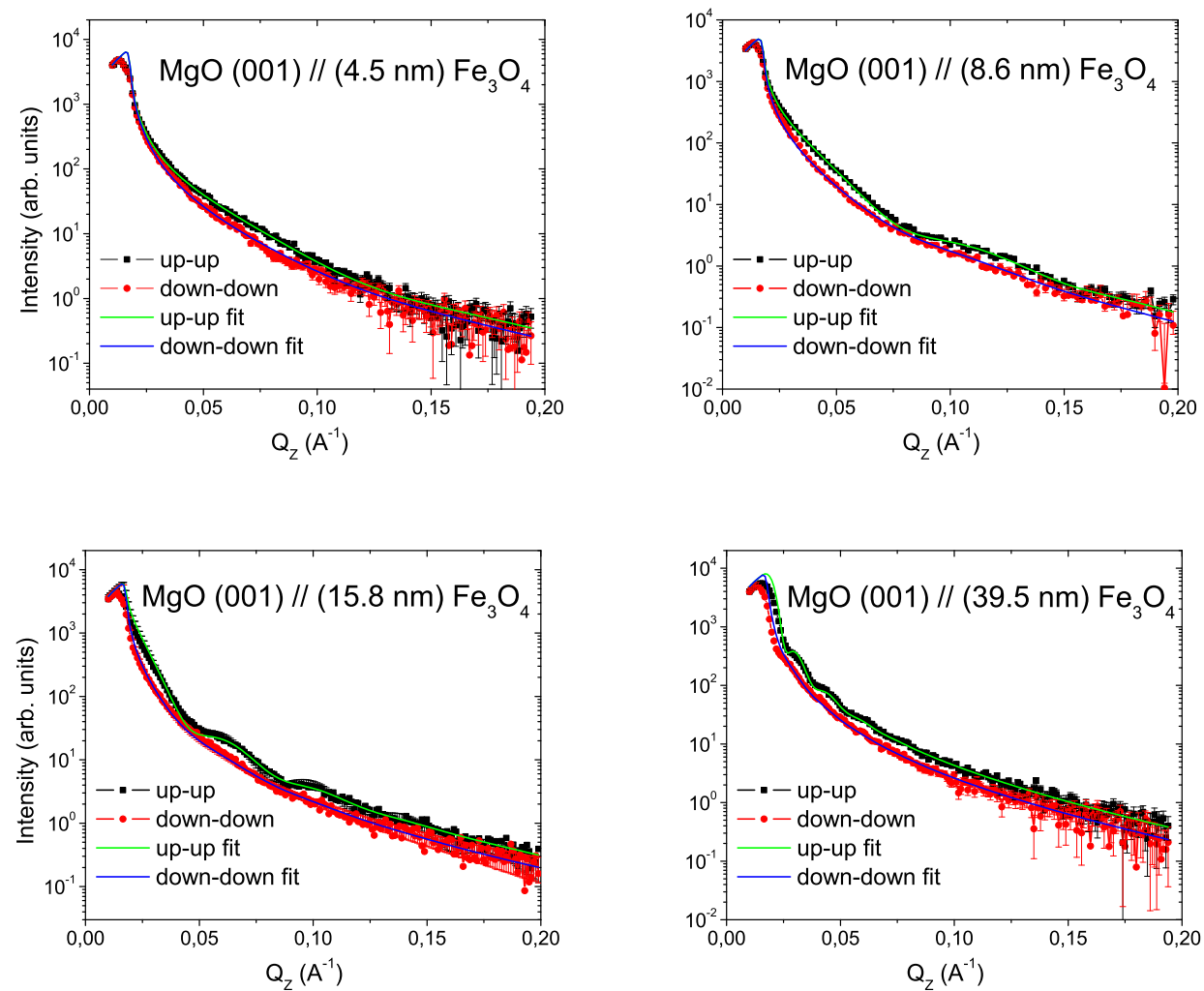

Figure 2.42: PNR measurements from magnetite films with different thicknesses at an applied magnetic field of 1 Tesla at room temperature. The dotted and squared curves correspond respectively to the reflection of up and down polarized neutrons, whereas the solid lines correspond to the fit. 
The measured data was adequately treated with $L A M P$ software in order so subtract both the superficial humidity and the background contribution, and fitted by means of GenX software [72]. The fits represented by solid lines in figure 2.42 were obtained by using film thickness, roughness and magnetic moment, and substrate roughness as variable parameters. Thicknesses (t) and surface roughnesses $(\sigma)$ parameters from the PNR fits are consistent with the previously performed XRR measurements (table 2.5).

The experimental data from PNR is well reproduced when the model used for the fit corresponds to a single layer with an homogeneous magnetic moment throughout the film. However, this is in contrast to the magnetic contribution from the film surface or from the interface of the film with the substrate suggested by Arora et al. [9, 10]. Indeed, the magnetic moment (m) obtained from the fit is always lower than the bulk value of $480 \mathrm{emu} / \mathrm{cm}^{3}$. While the 8 -nm-thick film, has a magnetization value of $553 \mathrm{emu} / \mathrm{cm}^{3}(115 \%$ of the bulk value) obtained from the VSM magnetometry measurement, the PNR data reveal a magnetization of $197 \mathrm{emu} / \mathrm{cm}^{3}$ (41\% of the bulk value). Regarding the 15 -nm-thick film, its saturation magnetization is lower than the bulk value in both sets of measurements but it is even lower in the case of PNR: 380 $\mathrm{emu} / \mathrm{cm}^{3}$ from VSM and $258 \mathrm{emu} / \mathrm{cm}^{3}$ from PNR.

Previous PNR studies in $\mathrm{Fe}_{3} \mathrm{O}_{4} / \mathrm{NiO}$ and $\mathrm{Fe}_{3} \mathrm{O}_{4} / \mathrm{CoO}$ exchange biased multilayers, where the thickness of the $\mathrm{Fe}_{3} \mathrm{O}_{4}$ layers are $15-16 \mathrm{~nm}$, yield a magnetic moment close to that of bulk in the center of the $\mathrm{Fe}_{3} \mathrm{O}_{4}$ layers and a noticeable reduction toward the interfaces [177, 178].

Indeed, the magnetic moments $(\mathrm{m})$ obtained from the fit are always lower than those from the VSM and SQUID measurements, but also, whereas from the VSM values, an increase in the magnetization with decreasing thicknesses is observed, from the PNR values the opposite behavior is found, in agreement with the other results reported previously in the literature [116, 119, 121, 129, 130, 142]. It is evident that the enhanced magnetic signal from the SQUID or VSM measurements cannot be due to any surface or interface magnetic contribution from the film. 


\subsubsection{X-ray absorption and magnetic circular dichroism}

To investigate the origin of the magnetic moment observed in the films we have also performed x-ray absorption spectroscopy (XAS) and XMCD experiments at the $L_{2,3} \mathrm{Fe}$ absorption edges. Two films, of $8 \mathrm{~nm}$ and $58 \mathrm{~nm}$ thickness, were measured at the ID08 beamline of the ESRF in Grenoble. The XMCD experiments were performed at $300 \mathrm{~K}$ under an applied field of $\pm 2.5 \mathrm{~T}$, with total electron yield detection and a $\sim 100 \%$ polarization rate.

In figure 2.43 (top panel), XAS curves are plotted as obtained from the average of the left- and right- circularly polarized light absorption, together with a bulk magnetite for comparison. Once normalized to the absorption edge jump obtained well above the edges $\left(E_{N} \sim 750 \mathrm{eV}\right)$ in a bulk single crystal sample [179], the white line for the films are slightly higher than that obtained in the bulk, indicating that the number of $3 d$ holes in the Fe $3 d$ band is somehow enhanced. This effect may be related with surface effects or APBs. The shape of the XAS curves of the films evidences the absence of any substantial amount of metallic iron in the films.

Figure 2.43 (low panel) shows the XMCD curves of both samples, which are in good agreement with the corresponding spectra of a variety of sizes of iron oxide nanoparticles reported by Park et al. [180]. The sum-rule analysis of the XMCD curves allows obtaining the Fe magnetic moments in the oxide films [181, 182].

The number of $\mathrm{Fe} 3 d$ holes used for the analysis (14.1 and 14 holes in the $3 \mathrm{~d}$ band per formula unit for the 8 and the $58 \mathrm{~nm}$ films respectively) has been estimated from the integral of the XAS white lines taking as reference the bulk value (13.5 holes/f.u.).

The magnetization obtained at $2.5 \mathrm{~T}$ in these two samples is $432 \mathrm{emu} / \mathrm{cm}^{3}$ (or $3.6 \mu_{B} / f . u$.) for the $58 \mathrm{~nm}$ film and $220 \mathrm{emu} / \mathrm{cm}^{3}$ for the $8 \mathrm{~nm}$ film (corresponding to $1.83 \mu_{B} / f . u$.), both in very good agreement with the PNR values (see table 2.42, Given the mean free path of a $710 \mathrm{eV}$ photon in magnetite $(\sim 150 \mathrm{~nm})$ and $\mathrm{MgO}(\sim 500 \mathrm{~nm})$, our result shows that the magnetic moment observed by XMCD is due to $\mathrm{Fe}_{3} \mathrm{O}_{4}$, as the films are probed across entirely, while only 1 in 2000 Fe impurities (for a $1 \mathrm{~mm}$ thick substrate) are excited by the x-ray beam before its total attenuation. Although a precise determination of the orbital to spin ratio in magnetite requires to extend the XMCD experiment in a much wider energy range [183], we estimate that $\mu_{L} / \mu_{S}<0.05$ in our samples. 


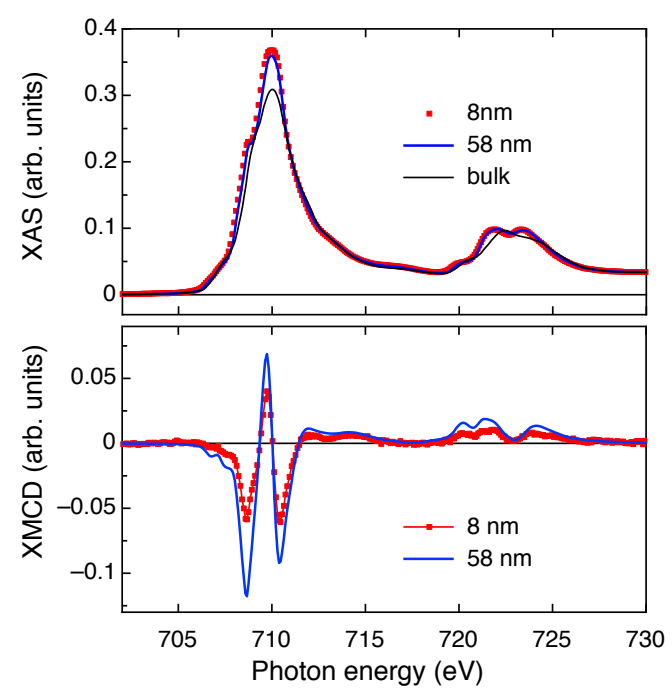

Figure 2.43: Top panel: XAS curves obtained from the average of the left- and rightcircularly polarized light absorption, together with a bulk magnetite. Down panel: XMCD experiment performed at $300 \mathrm{~K}$ under an applied magnetic field of $\pm 2.5 \mathrm{~T}$.

In the following table, a summary of the morphological and magnetic properties obtained with the different characterization techniques can be observed:

\begin{tabular}{||c|c||c|c|c||c||c||}
\hline \hline $\begin{array}{c}t_{X R R} \\
(\mathrm{~nm})\end{array}$ & $\begin{array}{c}t_{P N R} \\
(\mathrm{~nm})\end{array}$ & $\begin{array}{c}\sigma_{X R R} \\
(\mathrm{~nm})\end{array}$ & $\begin{array}{c}\sigma_{P N R} \\
(\mathrm{~nm})\end{array}$ & $\begin{array}{c}m_{V S M} \\
\mathrm{emu} / \mathrm{cm}^{3}\end{array}$ & $\begin{array}{c}m_{P N R} \\
\mathrm{emu} / \mathrm{cm}^{3}\end{array}$ & $\begin{array}{c}m_{X M C D} \\
\mathrm{emu} / \mathrm{cm}^{3}\end{array}$ \\
\hline \hline 4.5 & 4.5 & 0.4 & 0.3 & 1050 & 163 & - \\
\hline 8.0 & - & - & - & - & - & 220 \\
\hline 8.6 & 8.0 & 0.5 & 0.4 & 553 & 200 & - \\
\hline 15.8 & 15.0 & 0.4 & 1.9 & 380 & 258 & - \\
\hline 39.5 & 38.3 & 0.5 & 0.4 & 305 & 284 & - \\
\hline 58.0 & - & - & - & - & - & 432 \\
\hline \hline
\end{tabular}

Table 2.5: Comparison between thickness $(\mathrm{t})$ and roughness $(\sigma)$ values obtained from $\mathrm{XRR}$ and PNR, and magnetization $(\mathrm{m})$ from VSM at $1.8 \mathrm{~T}$, PNR at $1 \mathrm{~T}$, and XMCD at $2.5 \mathrm{~T}$.

The XMCD results agree with the behavior observed in PNR. However, In order to discern the existence of Fe clusters in the film, whose presence could lead to an enhanced magnetization that cannot be properly measured with PNR or XMCD, XPS where performed in selected magnetite thin films to determine the existence of minority parasitic phases in the magnetite films 


\subsubsection{X-ray Photoemission Spectroscopy measurements}

In order to discern the existence of Fe clusters in the film, whose presence could lead to an enhanced magnetization that cannot be properly measured with PNR or XMCD, XPS measurements were performed on the Fe $2 p$ corelevel spectrum in selected magnetite thin films.

The iron and its various oxides $\left(\mathrm{Fe}_{2} \mathrm{O}_{3}, \mathrm{Fe}_{3} \mathrm{O}_{4}\right.$ and $\mathrm{FeO}$ among others) can be distinguished from each other by their characteristic satellite peaks, which arise due to charge transfer screening [184, 185]. The $\mathrm{Fe} 2 p_{3 / 2}$ satellite binding energy is different for the $\mathrm{Fe}^{2+}$ or $\mathrm{Fe}^{3+}$ valence states: $\mathrm{BE}=715.5 \mathrm{eV}$ in $\mathrm{FeO}$ and $\mathrm{BE}=719 \mathrm{eV}$, in $\mathrm{Fe}_{2} \mathrm{O}_{3}$. In the case of $\mathrm{Fe}_{3} \mathrm{O}_{4}$ both satellites are present, which produces an unresolved peak.

Figure 2.44 shows the Fe 2p core level XPS spectra of $3 \mathrm{~nm}, 5 \mathrm{~nm}, 40 \mathrm{~nm}$ and $75 \mathrm{~nm}$ thick magnetite films, and the spectra from pure $\mathrm{Fe}_{3} \mathrm{O}_{4}, \mathrm{Fe}_{2} \mathrm{O}_{3}$ and Fe powder in the range of 705 to $735 \mathrm{eV}$. From the figure it is evident that the binding energy (BE) peak at $706.6 \mathrm{eV}$, characteristic of metallic Fe $2 p_{3 / 2}$ cannot be observed in any of our films, leading to the conclusion that there are not metallic Fe clusters and, definitely, the origin of the enhanced magnetic moment is not intrinsic to the magnetite film.

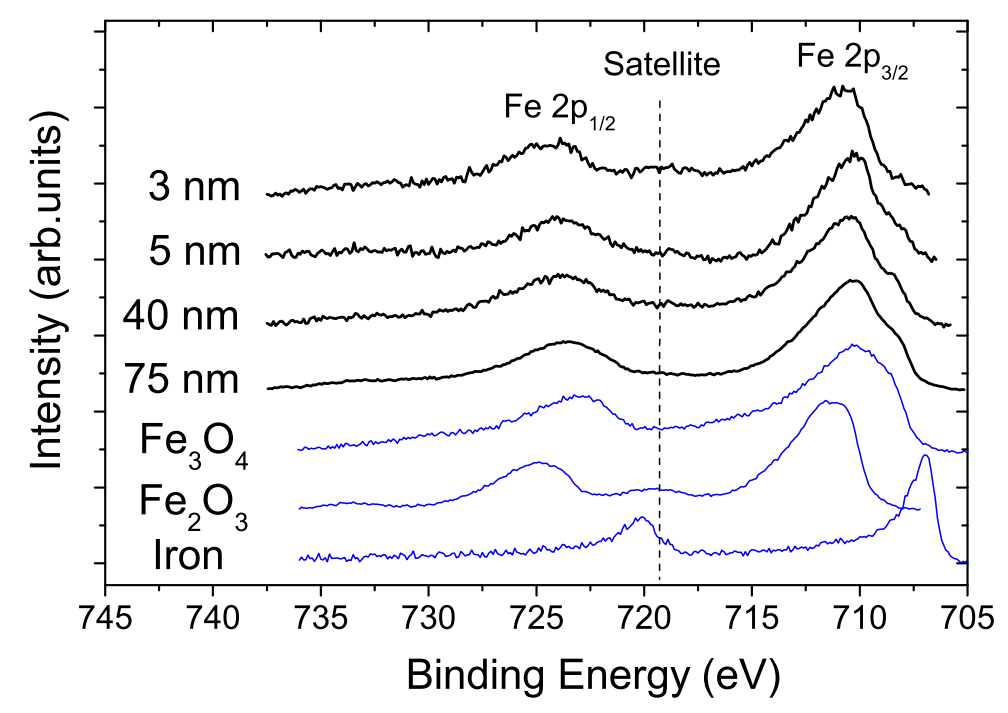

Figure 2.44: XPS measurements on the Fe $2 \mathrm{p}$ core level spectrum in magnetite thin films with different thicknesses. The data are plotted together with XPS spectrums of pure $\mathrm{Fe}_{2} \mathrm{O}_{3}, \mathrm{Fe}_{3} \mathrm{O}_{4}$ and $\mathrm{Fe}$. 
However, it is possible to observe that in the case of the thinner films (3 $\mathrm{nm}$ thick), the $2 p_{3 / 2}$ satellite increases its intensity and its position can be distinguished near $\mathrm{BE}=719 \mathrm{eV}$, indicating a deviation of the stoichiometry towards an $\mathrm{Fe}_{2} \mathrm{O}_{3}$ enriched phase which should lead to a decrease in the saturation magnetization, in opposition to the magnetic measurements performed through SQUID or VSM, but in agreement with PNR and XMCD results.

As a conclusion, it is proved that the origin of the enhanced magnetic moment measured by SQUID and VSM magnetometries cannot be found in the magnetite film, neither as a consequence to the noncompensation of spin moments between the tetrahedral and octahedral sublattices nor due to the presence of Fe clusters. Therefore, its origin might be related to impurities in the substrates or experimental errors.

\subsubsection{Origin of the enhanced magnetic moment.}

The possible sources of experimental error in the observation of nanoscale magnetism have been recently reported [186], as when measuring small signals (below $10^{-4} \mathrm{emu}$ ) magnetic traces introduced in our samples when handling them or due to impurities in the sample substrates must be considered. Therefore, as the enhanced magnetic moment was only found in the case of SQUID and VSM measurements, and PNR and XMCD are insensitive to the substrate, a study of the $\mathrm{MgO}$ substrates is strongly required.

ICP measurements of the $\mathrm{MgO}(001)(5 \mathrm{~mm} \times 5 \mathrm{~mm} \times 0.5 \mathrm{~mm})$ substrates, provided by our substrate commercial provider Crystal Gbmh, showed the existence of Fe impurities with a concentration $\leq 5 \mathrm{ppm}$. This concentration is enough to provide a magnetic signal of approximately $50.4 \mu e m u$ in each (5 $\mathrm{mm} \times 5 \mathrm{~mm} \times 0.5 \mathrm{~mm}$ ) substrate, and $290.3 \mu \mathrm{emu}$ in the case of the bigger substrates used for the PNR samples $(1 / 2 " \times 1 / 2 " \times 0.5 \mathrm{~mm})$. SQUID-RSO measurements were performed in a $\mathrm{MgO}(001)$ substrate in order to confirm the Fe impurities presence, and a typical hysteresis loop was obtained with a saturation magnetization of 11 eemu measured at a maximum magnetic field of 5 Tesla, which corresponds to a concentration of 1 ppm Fe impurities (figure 2.45).

It is possible to correct the saturation magnetization data by subtracting the substrate magnetic signal assuming a maximum Fe contribution of approximately $50 \mu е т u$. The resulting values show a decrease in the magnetization when decreasing magnetite films thicknesses (Figure 2.46), as reported previously in the literature [129, 130, 144, 153] 


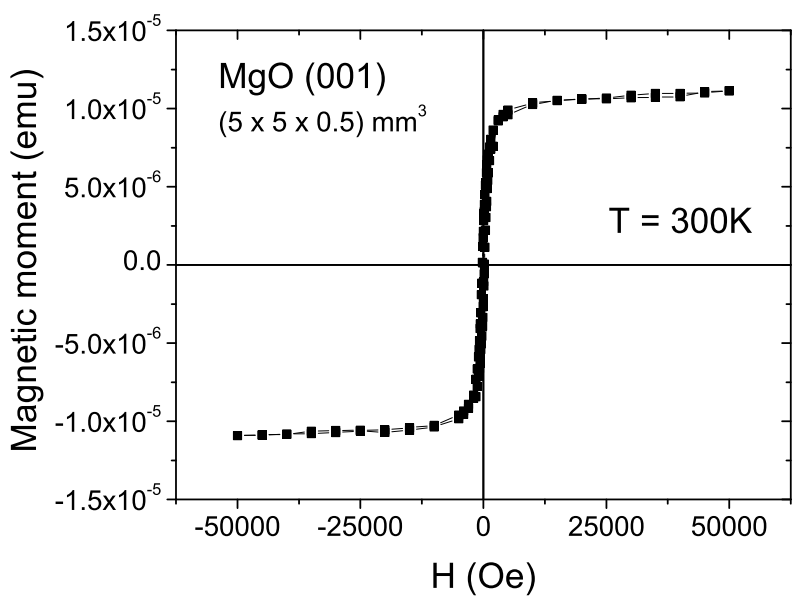

Figure 2.45: Magnetization of a standard as cast $\mathrm{MgO}(001)$ substrate $(5 \mathrm{~mm} \times 5$ $\mathrm{mm} \times 0.5 \mathrm{~mm})$ as a function of the applied magnetic field parallel to the surface at room temperature.

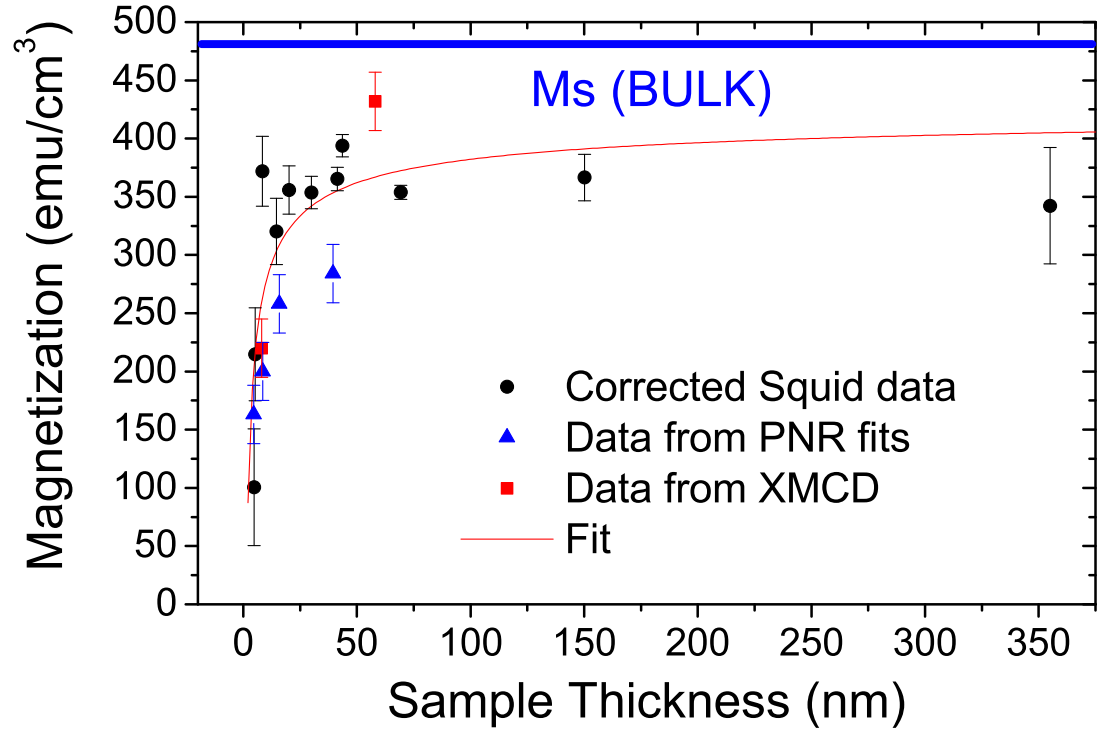

Figure 2.46: Magnetization as a function of the film thickness after the magnetic signal from the $\mathrm{MgO}$ (001) substrates was corrected. The red line represents a fit to the data using equation 2.18 
Therefore, the magnetization of the $\mathrm{Fe}_{3} \mathrm{O}_{4}$ films can be modeled as a layer with saturation magnetization Ms, which decreases as the film thickness ( $\mathrm{t}$ ) decreases. The corrected magnetization data (M) follow the same phenomenological law as that obtained for the Verwey transition temperature as a function of the film thickness (equation 2.8 in section 2.3.2):

$$
M=M_{S} \times\left(1-c \cdot t^{n}\right)
$$

If Ms, and c are taken as free parameters, the optimal fit is found when $n \approx$ -0.5 , and we obtain:

$$
\begin{gathered}
\mathrm{M}_{S}=430.7 \pm 35.2 \mathrm{emu} / \mathrm{cm}^{3} \\
\mathrm{c}=1.1 \pm 0.2 \mathrm{~nm}^{1 / 2}
\end{gathered}
$$

The $\mathrm{M}_{S}$ value obtained from the fit is $10 \%$ lower than the saturation magnetization reported for bulk magnetite $\left(480 \mathrm{emu} / \mathrm{cm}^{3}\right)$, which can be explained as a non saturation of the samples at the magnetic field where the saturation magnetizations were measured (5 T), due to the presence of APBs [129, 130]. As the APBs domain size (D) depends on the thickness of the magnetite films according to the relation given by equation 2.3 , the magnetization depends on D:

$$
M=M_{S} \times\left(1-\frac{d}{D}\right)
$$

Therefore, it can be understood that the magnetization of the films decreases with decreasing domain size, and thus with increasing APBs density.

The dependence of $\mathrm{M}_{S}$ with film thickness obtained after the substrate correction is in good agreement with the results obtained from PNR and XMCD experiments (Figure 2.46), supporting our assumption that the enhanced magnetic moment suggested by the magnetization measurements is indeed due to Fe impurities present in the $\mathrm{MgO}$ substrates.

It is important to stress that the Fe impurities are present in the as-received substrates and not implanted during the PLD deposition of $\mathrm{Fe}_{3} \mathrm{O}_{4}$. It is known that the kinetic energy of the impinging species in PLD typically lie in the 1$100 \mathrm{eV}$ range [20, 59]. Nevertheless, at the low fluence used, and given the high crystallinity, low roughness, and sharp interfaces of our films, we can estimate the energy of the species arriving at the substrate being $<5 \mathrm{eV}$ or lower. In this range of energy, implantation in the $\mathrm{MgO}$ is negligible [187]. Studies of $\mathrm{Fe}$ implantation in $\mathrm{MgO}$ typically require energies in the range of $50-100 \mathrm{keV}$ or higher [188, 189].

Another interesting possibility is the existence of $\mathrm{d}_{0}$ ferromagnetism in $\mathrm{MgO}$ as suggested recently in both nanoparticles [190, 191], and thin films [192]. We have not found any evidence of $\mathrm{Mg}$ vacancies in our single crystalline substrates and, therefore, this effect may be ruled out. 
As a conclusion, we obtained contradictory results in magnetization saturation of ultra-thin magnetite films when comparing SQUID and VSM magnetometry with PNR and XMCD measurements. However, XPS experiments showed the absence of metallic Fe impurities in the magnetite films, whose presence could justify the enhanced magnetic moment found through SQUID and VSM measurements.

Finally, ICP and SQUID magnetometry measurements performed in standard $\mathrm{MgO}$ (001) substrates showed the presence of Fe impurities in the substrates, and by subtracting its magnetic signal, the corrected thin films magnetizations showed a decrease with decreasing film thickness as $\propto t^{-1 / 2}$, which can be related to APBs density. This result together with the PNR and XMCD experiments indicate that the origin of the enhanced magnetic moment is extrinsic, due to the presence of Fe impurities in the $\mathrm{MgO}$ substrates, and not intrinsic to $\mathrm{Fe}_{3} \mathrm{O}_{4}$ [175]. 


\subsection{Study of the $\mathrm{Nb}: \mathrm{SrTiO}_{3} / / \mathrm{Fe}_{3} \mathrm{O}_{4}$ Schottky bar- rier}

The injection and detection of spin-polarized currents has attracted researchers interest due to its direct applications in spintronic devices, specially from the recent demonstration that spin injection can be obtained by tunneling through a barrier interface from a ferromagnetic half-metal into a semiconductor [193]. With this purpose, different types of tunnel contacts have been successfully used, such as STM tips [194], and Schottky barriers [195]. However, the growth of half-metallic ferromagnets onto semiconductors is not trivial, and very often the spin polarization is determined by disordered interfacial layers more than by the bulk properties. Therefore, great effort have been directed to the study of the necessary high quality, atomically flat surfaces.

Magnetite is an attractive spin-polarized source due to its predicted halfmetallicity. On the other hand, strontium titanate, $\mathrm{SrTiO}_{3}$ (tausonite) is a perovskite with a Pm3m space group, lattice parameter of $3.905 \AA$ and a density of $5.12 \mathrm{~g} / \mathrm{cm}^{3}$ whose transport properties can be tunned by doping. $\mathrm{SrTiO}_{3}$ (STO) is an insulator at room temperature [196], but through impurity doping (for example with $\mathrm{Nb}, \mathrm{La}$ or In) it can behave as a n-type semiconductor [197], a p-type semiconductor [198], and can even became superconductor at subKelvin temperatures [199], which makes Nb-doped $\mathrm{SrTiO}_{3}$ (Nb:STO) suitable as back electrode for an $\mathrm{Fe}_{3} \mathrm{O}_{4}$ - based spintronics device.

\begin{tabular}{||c||c|c|c|c||c||}
\hline \hline$\% \mathrm{Nb}: \mathrm{SrTiO}_{3}$ & $1.0 \%$ & $0.7 \%$ & $0.4 \%$ & $0.1 \%$ & $0 \%$ \\
\hline Resistivity $(\Omega \mathrm{cm})$ & 0.0035 & 0.0070 & 0.0500 & 0.0800 & $>10^{7}$ \\
\hline Mobility $\left(\mathrm{cm}^{2} / V s\right)$ & 9.0 & 8.5 & 8.5 & 6.5 & \\
\hline$e^{-}$concentration $\left(\mathrm{cm}^{3}\right)$ & & & $10^{21}$ & & $<10^{18}$ \\
\hline \hline
\end{tabular}

Table 2.6: Typical transport characteristics of $\mathrm{Nb}: \mathrm{SrTiO}_{3}$ as a function of the $\% \mathrm{Nb}$ [200].

Therefore, we have studied the bilayer system consisting on a magnetite layer on conducting Nb-doped $\mathrm{SrTiO}_{3}$ (001) $5 \times 5 \times 0.5 \mathrm{~mm}^{3}$ monocrystalline substrates, with different $\mathrm{Fe}_{3} \mathrm{O}_{4}$ thicknesses and two Nb-doping concentration: $1.4 \%$ and $0.1 \%$. The magnetite films have been grown with the optimized deposition conditions described in section 2.2 on the conductive Nb:STO substrates. Finally, structural, magnetic and temperature dependent electrical transport systematical characterization was performed. 
Standard XRD measurements were performed in all the magnetite samples deposited on $\mathrm{Nb}: \mathrm{STO}$ substrates in order to determine its crystallographic orientation growth. A standard symmetric $\theta-2 \theta$ X-ray experiment performed on a $33.6 \mathrm{~nm}$ magnetite film deposited on $0.1 \% \mathrm{Nb}: \mathrm{SrTiO}_{3}(001)$ substrate can be seen in figure 2.47, where $\mathrm{Fe}_{3} \mathrm{O}_{4}$ [001] peaks appear, showing the epitaxial growth of $\mathrm{Fe}_{3} \mathrm{O}_{4}(001)$ on the $\mathrm{STO}$ substrate.

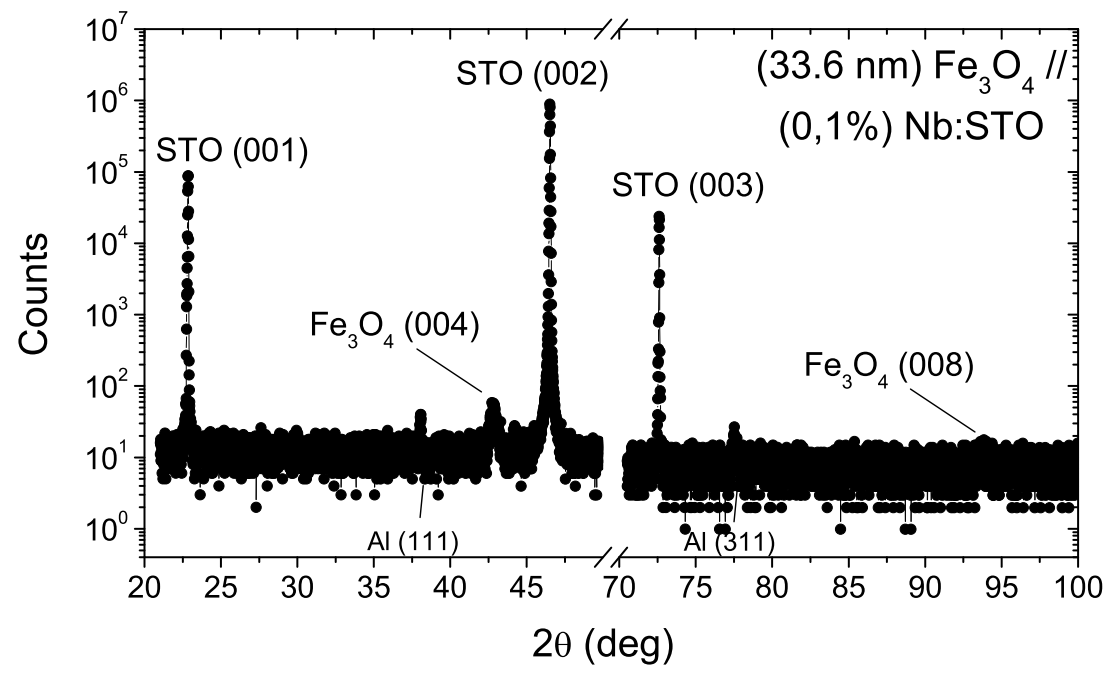

Figure 2.47: $\theta-2 \theta$ of a $33.6 \mathrm{~nm}$ magnetite film deposited on a $0.1 \% \mathrm{Nb}: \mathrm{SrTiO}_{3}$ substrate. The peaks indexed in blue come from the sample holder of the XRD equipment.

However, the Laue oscillations present when growing magnetite films on $\mathrm{MgO}$ (001) substrates are not present, revealing that the $\mathrm{Fe}_{3} \mathrm{O}_{4}$ films grown on STO substrates have a worse crystalline coherence. Indeed, the mismatch between the magnetite and the STO substrates is about $7.5 \%$.

The magnetic characterization was performed by means of SQUID magnetometry. Magnetization measurements as a function of the applied magnetic field at room temperature (figure 2.48) show a magnetization saturation of $435 \mathrm{emu} / \mathrm{cm}^{3}$, a $91 \%$ of the bulk value, revealing the low presence of APBs [129, 130] and the stoichiometric quality of the film. The stoichiometry and absence of epitaxial strain is also evidenced by the Verwey transition temperature extracted from the magnetization measurement as a function of the temperature in an applied magnetic field of 500 Oe [90, 106, 118, 122, 124, 126, 128], as is equal to the bulk value: $\mathrm{T}_{V}=120 \mathrm{~K}$ (see figure 2.49). 


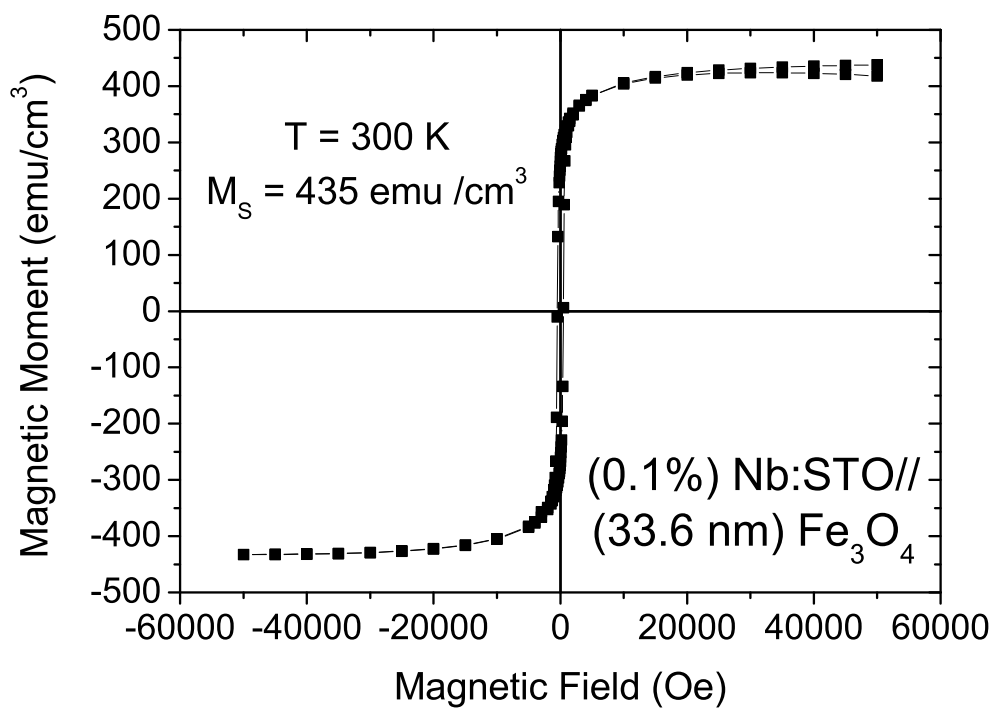

Figure 2.48: Magnetization as a function of the applied magnetic field at $300 \mathrm{~K}$ of a $32.6 \mathrm{~nm}$ magnetite film deposited on a $0.1 \% \mathrm{Nb}: \mathrm{STO}$ substrate.

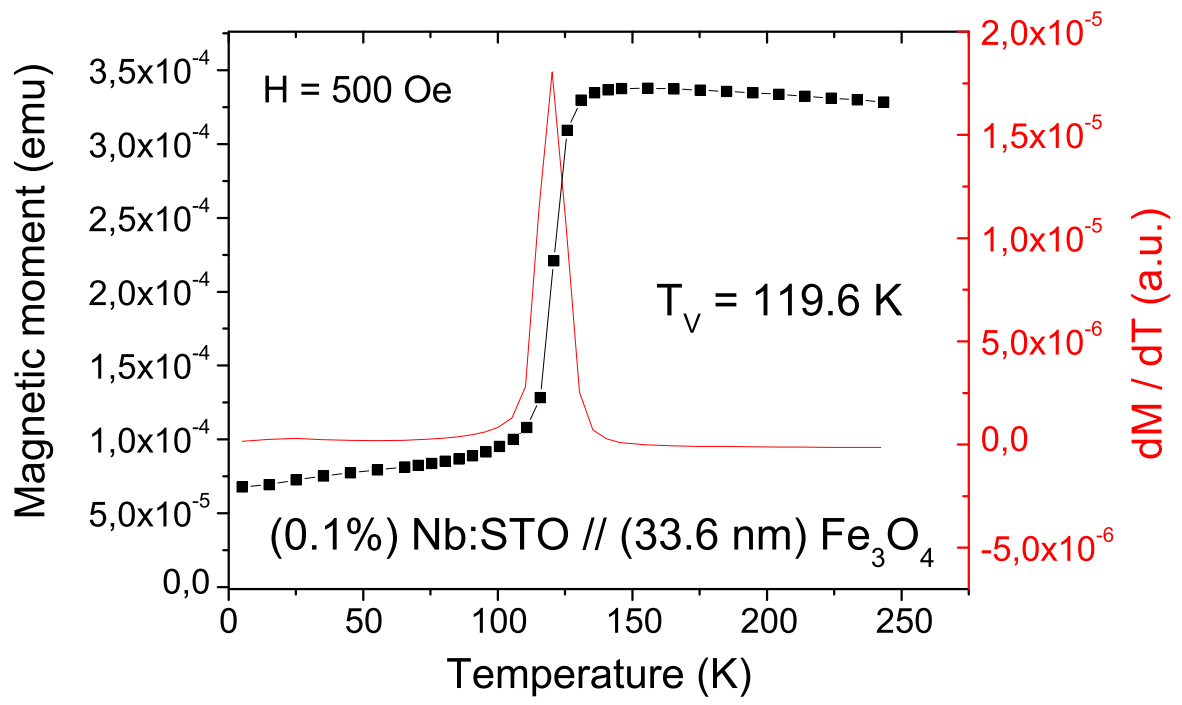

Figure 2.49: Magnetization as a function of the temperature in an applied field of 500 Oe of a $32.6 \mathrm{~nm}$ magnetite film deposited on a $0.1 \% \mathrm{Nb}$ :STO substrate. 
Current-voltage characteristics were measured with the conventional fourprobe method with the current perpendicular to the interface with the contact configuration shown in figure 2.50. The samples were contacted with indium contacts of sizes between $1 \times 1 \mathrm{~mm}^{2}$ and $2 \times 2 \mathrm{~mm}^{2}$ on the backside of the substrate, and silver paint contacts of sizes less than $1 \times 1 \mathrm{~mm}^{2}$ on the magnetite film surface.

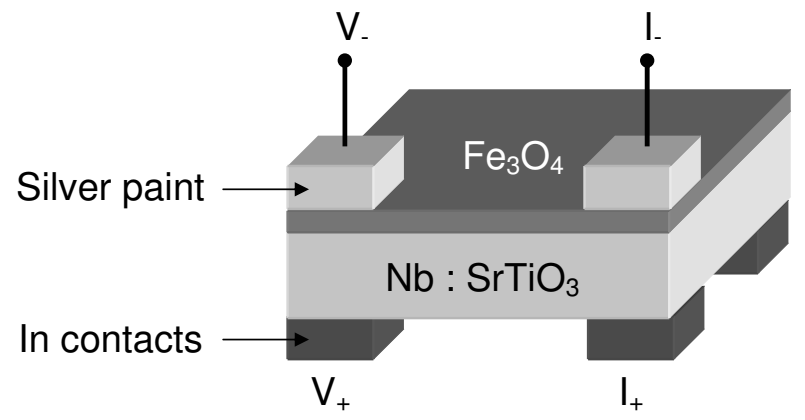

Figure 2.50:

Sketch of the contact configuration. The forward bias is defined with the positive voltage applied to the Nb:STO substrate.

The current-voltage (I-V) characteristics of the Nb:STO // $\mathrm{Fe}_{3} \mathrm{O}_{4}$ interface measured at zero applied magnetic field for the lower doping concentration $(0.1 \% \mathrm{Nb})$ is shown in figure 2.51

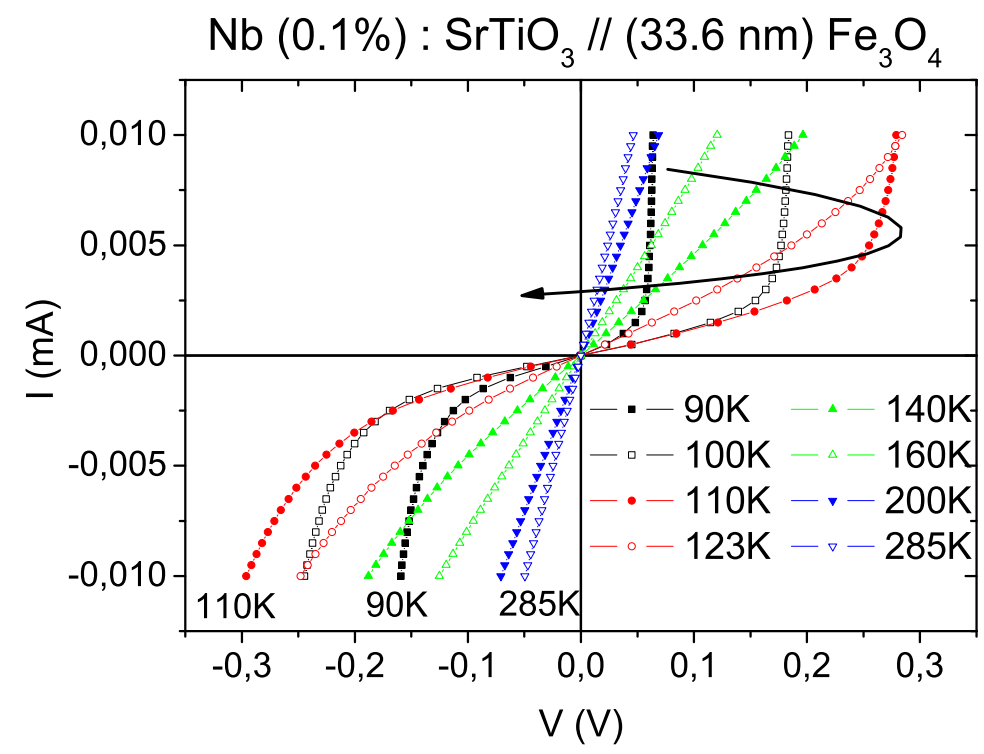

Figure 2.51: I-V characteristics of the $(0.1 \%) \mathrm{Nb}: \mathrm{SrTiO}_{3} / / \mathrm{Fe}_{3} \mathrm{O}_{4}$ Schottky barrier diodes in zero magnetic field. 
Due to the formation of a Schottky barrier at the interface between the magnetite and the substrate, the current-voltage characteristics are nonlinear and asymmetric [201], which indicates that the Nb:STO acts as a metal and magnetite as a semiconductor, being consistent with both the metallic conductivity of the substrate and the band structure of magnetite. It can be observed that the rectifying characteristics are limited in the temperature range between 90 and 120K, whereas at higher temperatures its I-V shows almost linear (ohmic) behavior.

The out of plane I-V data can be analyzed within a thermoionic emission model with an ohmic term $(\mathrm{V} / \mathrm{R})$ introduced to account the leakage currents, which is expected to have a small contribution [201]:

$$
I=I_{S}\left[\exp \left(\frac{e V}{\eta k_{B} T}\right)-1\right]+\frac{V}{R}
$$

$\mathrm{R}$, the ideality factor $\eta$ and the reverse saturation current $\mathrm{I}_{S}$ are taken as free parameters. The ideality factors ranged from 1 at low to 6 at high temperatures, in agreement with the values obtained previously in literature by other authors [201].

The Schottky barrier height in zero magnetic field can be determined from the reverse saturation current $I_{S}$ value, taking into account that:

$$
I_{S}=S A^{*} T^{2} \exp \left(\frac{-e \Phi_{B}}{k_{B} T}\right)
$$

where $\mathrm{S}$ denotes the junction area, e the electronic charge, $\Phi$ the Schottky barrier height, $\mathrm{k}_{B}$ the Boltzmann constant and $\mathrm{A}^{*}$ the Richardson constant, defined as:

$$
A^{*}=\frac{e m^{*} k_{B}^{2}}{2 \pi^{2} \hbar^{3}}
$$

being $\mathrm{m}^{*}$ the effective mass and $\hbar$ Planck's constant.

However, as the junction area is a parameter that may not be properly estimated, it is preferable to obtain the Schottky barrier height from $\mathrm{I}_{S}$ dependence with the temperature, considering two different regimes above and below the Verwey transition temperature as can be seen in figure 2.52. The Schottky barrier height values obtained were $\Phi_{B}=0.07 \pm 0.01 \mathrm{eV}$ below the Verwey transition and $\Phi_{B}=0.71 \pm 0, .17 \mathrm{eV}$ above the transition, in agreement with the values reported in literature by other authors [201, 202]. The obtained results indicate that a well defined Schottky barrier is formed below the Verwey temperature, where a gap opens at the Fermi level in the electronic structure of magnetite. However, above the Verwey transition temperature, the weak nonlinearity in the current-voltage curves might be due to some tunneling process [201], which should be further investigated. 


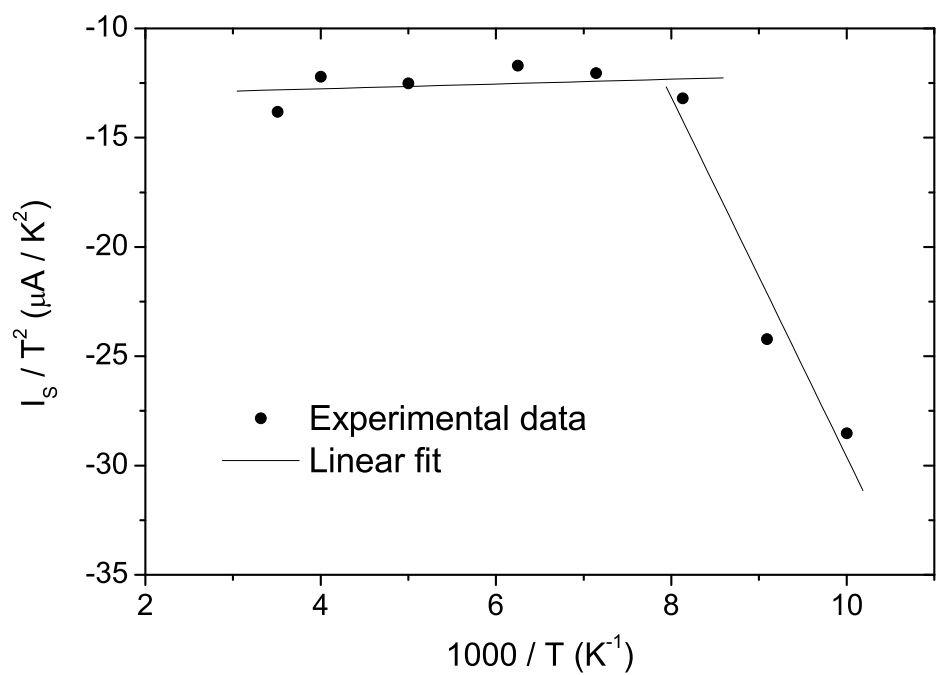

Figure 2.52: Reverse saturation current $\mathrm{I}_{S}$ divided by the square of the absolute temperature of the $0.1 \% \mathrm{Nb}: \mathrm{STO} / / \mathrm{Fe}_{3} \mathrm{O}_{4}$ Schottky barrier.

Concerning the magnetite films deposited on (1.4\%)Nb:STO substrates with the higher doping concentration, as the film thickness is decreased, the non-linearity in I-V appears for higher temperatures, enhancing the diode behavior at high temperature (Figure 2.53). For the heterojunctions with all magnetite thicknesses but the $3.9 \mathrm{~nm}$ thick, the I-V characteristics shift towards higher forward bias voltage up to a maximum value upon lowering temperature, and below a certain temperature, the tendency is reversed with a shift of the I-V characteristic towards lower forward voltage.

However, it is observed that the maximum forward bias of the magnetite films deposited on $0.1 \% \mathrm{Nb}: \mathrm{SrTiO}_{3}$ is higher than the value obtained for the samples grown on $1.4 \% \mathrm{Nb}: \mathrm{SrTiO}_{3}$ substrates. Theoretical calculations performed recently by Han et al. in perovskite oxide heterostructures [203], have attributed this behavior to the Schottky junction formed by the In contact and the $\mathrm{Nb}: \mathrm{SrTiO}_{3}$ substrate. This effect decreases the maximum bias voltage for higher doping densities and the experimental measurements cannot be fitted with the previous model. Therefore, a study considering contribution from direct tunneling or temperature-assisted tunneling as well as In-STO:Nb contacts contribution requires further analysis. 

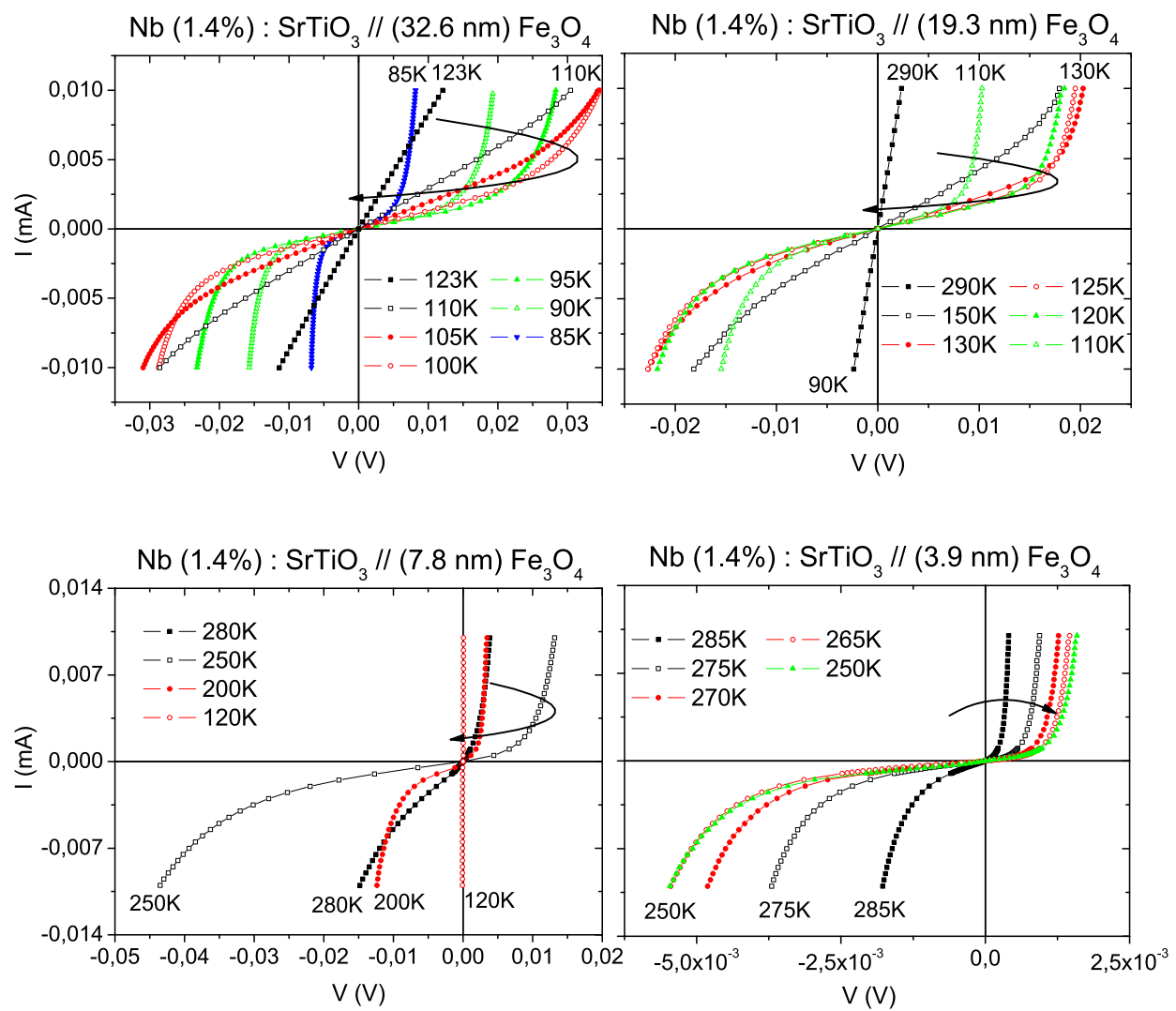

Figure 2.53: I-V characteristics of the $1.4 \% \mathrm{Nb}: \mathrm{STO} / / \mathrm{Fe}_{3} \mathrm{O}_{4}$ Schottky barrier diodes under magnetic field of $0 \mathrm{~T}$. 


\section{Chapter 3}

\section{$\mathrm{Sr}_{2} \mathrm{CrReO}_{6}$ epitaxial thin films}

$\mathrm{Sr}_{2} \mathrm{CrReO}_{6}$ (SCRO) is a ferromagnetic material which belongs to the family of the double perovskite oxides. This system has a great interest in spintronics due to its high spin polarization and high Curie temperature, indeed, it has the highest $\mathrm{T}_{C}$ among double perovskites: $635 \mathrm{~K}$ [204]. However, it has a complex crystalline structure which strongly influences its magnetic and transport properties, making the growth of high quality epitaxial crystalline SCRO films a challenge.

In this chapter, a brief introduction to double perovskites precedes the description of the exhaustive experimental research developed in order to optimize the SCRO growth on STO (001) substrates by means of PLD. Once the optimal deposition conditions were found, the double perovskite films were completely characterized demonstrating the high quality of our films from the structural, magnetic and transport point of view.

\subsection{An introduction to $\mathrm{Sr}_{2} \mathrm{CrReO}_{6}$}

\subsubsection{Double Ordered Perovskites}

Double ordered perovskites are oxides with the general chemical formula $\mathrm{A}_{2} \mathrm{BB}^{\prime} \mathrm{O}_{6}$, where $\mathrm{A}$ is an alkaline or rare earth ion, and B and B' are transition metals. Their investigation dates from 1961, when Longo and Ward reported the ferromagnetic behavior of Re based perovskites with the general formula $\mathrm{A}_{2} \mathrm{BReO}_{6}$ [205]. 
In Fe-based double ordered perovskites, with general formula $\mathrm{A}_{2} \mathrm{FeB}^{\prime} \mathrm{O}_{6}(\mathrm{~A}$ $\left.=\mathrm{Sr}, \mathrm{Ca}, \mathrm{Ba} ; \mathrm{B}^{\prime}=\mathrm{Mo}, \mathrm{Re}\right), \mathrm{Fe}^{3+}$ and B' lattices couple antiferromagnetically. These materials have been reported to have half-metallicity and high Curie temperatures, which makes them interesting for their potential applications at room temperature in spin electronics. What is more, in 1998 Kobayashi et al. reported that polycrystalline samples of the metallic ferrimagnet $\mathrm{Sr}_{2} \mathrm{FeMoO}_{6}$ with the Curie temperature $\mathrm{T}_{C} \approx 420 \mathrm{~K}$ showed sharp low-field tunnel magnetoresistance not only at low temperatures, but even at room temperature due to grain boundary effects, which was explained by means of electronic-structure calculations that indicated the half-metallicity of the material [206]. One year later, Kobayashi reported that $\mathrm{Sr}_{2} \mathrm{FeReO}_{6}$ showed a similar TMR characteristic to $\mathrm{Sr}_{2} \mathrm{FeMoO}_{6}$ [207], and in the next years other Fe-based ordered double perovskites were also reported to have a half-metallic nature and a high $\mathrm{T}_{C}$, such as $\mathrm{Ba}_{2} \mathrm{FeMoO}_{6}$ [208] and $\mathrm{Ca}_{2} \mathrm{FeMoO}_{6}$ [209].

Therefore, and due to their potential application in the new-born field of spintronics [210], the study of structural and magnetic properties as well as electronic structure of Fe-based double perovskites became a research field of general interest [211].

Concerning Cr-based double perovskites with the general formula $\mathrm{A}_{2} \mathrm{CrB}^{\prime} \mathrm{O}_{6}$, they have also been found to show ferromagnetic of ferrimagnetic properties being the $\mathrm{Cr}^{3+}$ and B' sublaticces antiferromagnetically coupled. The first reported works in this materials date from 1960's [212], and more recently $\mathrm{Sr}_{2} \mathrm{CrMoO}_{6}$ and $\mathrm{Sr}_{2} \mathrm{CrWO}_{6}$ have shown to have tunneling-type magnetoresistance [213, 214]. However, few literature can be found about $\mathrm{Sr}_{2} \mathrm{CrReO}_{6}$ (SCRO) polycrystalline bulk and epitaxial thin films.

The crystalline structure of double ordered perovskites is a modified perovskite structure $\left(\mathrm{ABO}_{3}\right)$ where the $\mathrm{BO}_{6}$ and $\mathrm{B}^{\prime} \mathrm{O}_{6}$ octahedra are alternately arranged in two interleaving fcc sublattices antiferromagnetically coupled [211]. The ideal perovskite structure is cubic, being described within de Fm3m space group. However, this structure is often distorted leading to a tetragonal structure (I4/m space group), which requires a new unit cell to be defined with its main axes defined along the cubic [110] and [110] directions. When the tetragonal distortion takes place, a pseudocubic unit cell can be defined in order to facilitate the experimental work, for example, the XRD peaks indexation. However, in this chapter all the double perovskite Bragg reflections will be indexed in the tetragonal $\mathrm{I} 4 / \mathrm{m}$ space group. 


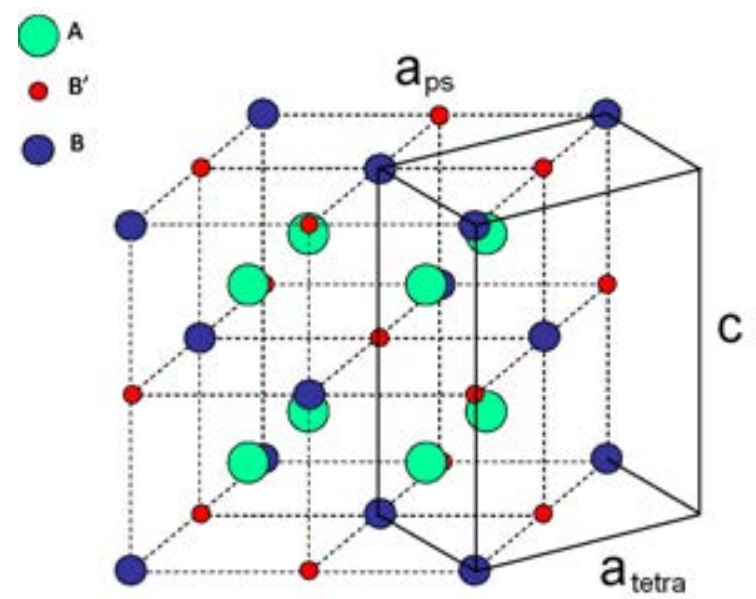

Figure 3.1: Structure of an ideal cubic double perovskite with chemical formula $\mathrm{A}_{2} \mathrm{BB}^{\prime} \mathrm{O}_{6}$. Oxygen atoms are located at the octahedra vertex and $\mathrm{B}$ and $\mathrm{B}$ ' atoms are alternated. The thick line defines the tetragonal cell, whereas the dashed line indicates the cubic or pseudocubic unit cell [211].

As can be seen in figure 3.1, the relation between the lattice parameters of the tetragonal (tetra) and the pseudocubic ( $p s)$ cells is:

$$
\begin{aligned}
c & =c_{\text {tetra }}=c_{p s} \\
a & =b=a_{\text {tetra }}=\frac{a_{p s}}{\sqrt{2}}
\end{aligned}
$$

\subsubsection{Crystallographic structure defects: Antisites (AS)}

Due to their complex crystalline structure, the synthesis of high quality double ordered perovskites is hard to achieve. Usually, these materials present defects such as oxygen vacancies, and more often they have the so-called antisite defects (AS), consisting on misplaced B ions which occupies the B' sites and vice versa which strongly influence the double perovskite physical properties.

By means of Monte Carlo simulations, Ogale et al. [215] predicted that defects such as $\mathrm{Fe} / \mathrm{Mo}$ disorder in the $\mathrm{Sr}_{2} \mathrm{FeMoO}_{6}$ double perovskite and oxygen vacancies can decrease dramatically both the Curie temperature and the magnetic moment. Taking into account that these structural defects affect the local magnetic structure, two possible situations can be found: either the misplaced ion conserves its magnetic moment direction respecting the perfect site, or it acquires the direction expected at the new location, therefore, inverting its magnetic moment direction, as the sublattices are antiferromagnetically coupled. 
By first-principles band structure calculations and a tight-binding approach, Solovyev investigated the local stability of the half-metallic ferrimagnetic (FiM) states in Fe-based double perovskites $\mathrm{Sr}_{2} \mathrm{FeMO}_{6}(\mathrm{M}=\mathrm{Mo}$, Re, and W) [216], finding that FiM phase is unstable and cannot be stabilized by purely electronic mechanisms. It was found that, in partially disordered compounds, the FiM ordering could be stabilized by less than $10 \%$ antisite defects due to the antiferromagnetic super-exchange interactions between Fe atoms located at the ideal and at the antisite positions. As the antisite defects will deteriorate mobility of the electrons, it might explain the fact that some of the ferrimagnetically ordered DP, like $\mathrm{Ca}_{2} \mathrm{FeReO}_{6}$, are insulators. Therefore, there should be some optimal concentration of the antisite defect, which would be sufficiently large to stabilize the FiM phase and low enough to preserve mobility of the electrons and rather high saturation magnetization, required for the magnetoresistive behavior.

As a conclusion, different defect configurations lead to different magnetic moments at different crystallographic sites, producing an overall magnetization of the material that will depend on defect concentration, as well as on their spatial distribution.

Balcells et al. [217] showed that the concentration of antisite defects in $\mathrm{Sr}_{2} \mathrm{FeMoO}_{6}$ could be controlled by the synthesis protocol, and observed a variation of the saturation magnetization $\left(\mathrm{M}_{S}\right)$ with $\mathrm{AS}$, providing an experimental confirmation of the ferrimagnetic ordering in the $\mathrm{Sr}_{2} \mathrm{FeMoO}_{6}$ lattice. They proposed an experimental formula giving its dependence with AS and doping for this double perovskite:

$$
\begin{aligned}
M_{S} & =m_{B}-m_{B^{\prime}} \\
& =(1-2 w) m_{F e}-(1-2 w) m_{M o}
\end{aligned}
$$

where $\mathrm{m}_{B}$ and $\mathrm{m}_{B^{\prime}}$ are the magnetizations of the $\mathrm{B}$ and $\mathrm{B}^{\prime}$ sublattices, $\mathrm{m}_{\mathrm{Fe}}$ and $\mathrm{m}_{M o}$ are the magnetic moment of the Fe and Mo ions respectively, and $w$ is the AS concentration.

If we introduce the parameter $\mathrm{M}_{S T}$ as the saturation magnetization in the case of perfect crystallographic ordering, the equation 3.2 can be rewritten as:

$$
M_{S}=M_{S T}(1-2 w)
$$

In both equations the parameter $w$ indicates the percentage of AS defects, meaning $0 \%$ a defect-free structure fully ordered and $50 \%$ a fully disordered double perovskite. If $\mathrm{B}[\mathrm{B}]$ and $\mathrm{B}$ '[B] are the number of $\mathrm{B}$ and $\mathrm{B}$ ' atoms occupying $\mathrm{B}$ sites, the percentage of $\mathrm{AS}$ can be calculated as:

$$
A S(\%)=\frac{B^{\prime}[B]}{B[B]+B^{\prime}[B]} \cdot 100 \%
$$


Therefore, the concentration of AS can be estimated from the magnetization data, but also from diffraction experiments, as, apart from the Bragg reflections due to the crystallographic structure, some superstructure peaks forbidden by symmetry, are visible in the double ordered perovskites. The less AS, the stronger such reflections are, disappearing for the completely disordered case when $\mathrm{AS}=50 \%$.

\subsubsection{Growth of epitaxial $\mathrm{Sr}_{2} \mathrm{CrReO}_{6}$ thin films}

Of all double perovskite oxides, we decided to focus on SCRO films due to its interest in spintronics, as was shown to have the highest Curie temperature 204]. However, if the growth of crystalline bulk double perovskites is a hard issue to deal with due to their complex structure, the growth of high quality epitaxial crystalline films is ever more difficult, and there was little reported information regarding SCRO films.

This topic represents an interesting challenge, not only from the spintronics perspective, but also from the film growth point of view which, after a thorough experimental work we finally overcame.

SCRO has a theoretical spin-only ionic saturation magnetization of $1 \mu_{B} / f . u$. but the reported experimental value is higher, $1.38 \mu_{B} / f . u$. [218], which has been explained by the large orbital contribution to the magnetization. However, as it was detailed in section 3.1.2, the saturation magnetization is reduced by the existence of antisite (AS) defects at the B/B' sites, which could also destroy the half-metallic nature of the material [211]. Therefore, the controllable preparation of high-quality epitaxial films of SCRO ordered double-perovskite with high $\mathrm{T}_{C}$ and full magnetization is highly desirable for electrodes in spintronic devices.

Most of the reported double perovskite thin film growth efforts have been devoted to the well-studied $\mathrm{Sr}_{2} \mathrm{FeMoO}_{6}$ (SFMO) material [219 223], and to a similar double-perovskite, $\mathrm{Sr}_{2} \mathrm{CrWO}_{6}(\mathrm{SCWO})$ with a lower $\mathrm{T}_{C}(473 \mathrm{~K})$ [224]. However, little information about the growth of SCRO thin films can be found in literature. Initially, Asano et al. reported the growth of SCRO films by magnetron sputtering [225] on STO substrates. They achieved epitaxial films and reported a significant change in coercivity from 100 Oe to $1 \mathrm{kOe}$ just by increasing the substrate temperature by $100{ }^{\circ} \mathrm{C}$, which was attributed to important changes in the surface morphology and roughness. Very recently, Geprägs et al., reported the growth of epitaxial SCRO films on STO substrates by means of PLD [226], with a higher crystalline quality than those from Asano. 


\subsection{Growth optimization}

We optimized the growth conditions of $\mathrm{Sr}_{2} \mathrm{CrReO}_{6}$ films on STO substrates by means of PLD. The use of pure targets is recommendable to obtain high quality films with this deposition technique, therefore, the sinterization and characterization of the SCRO target is the first step.

\subsubsection{Target preparation and characterization}

In order to obtain the desired $\mathrm{Sr}_{2} \mathrm{CrReO}_{6}$ stoichiometry, $\mathrm{SrCO}_{3}, \mathrm{Cr}_{2} \mathrm{O}_{3}, \mathrm{ReO}_{3}$ and Re powders were used as precursors for the target preparation by solidstate reaction.

The precursors were annealed at $1375{ }^{\circ} \mathrm{C}$ for two hours in $\mathrm{Ar} / \mathrm{H}_{2}$ atmosphere at a concentration of approximately $98 / 2$. This treatment had to be repeated several times grounding the sample and performing structural and magnetic characterization in between until the desired stoichiometry was obtained. The powder was then pressed into a pellet, which was structurally and magnetically characterized by means of XRD and SQUID magnetometry, respectively, in order to guarantee the target quality before loading it into the process chamber and starting the thin film growth optimization process.

The XRD measurement was performed at room temperature and the data was analyzed with Rietveld method by means of FullProf (figure 3.2), demonstrating that there was only crystalline $\mathrm{Sr}_{2} \mathrm{CrReO}_{6}$ with an AS concentration of approximately 15-17\%, without any parasitic phases which could have remained.

The target magnetization measurements as a function of the applied magnetic field were performed by means of SQUID magnetometry at $4 \mathrm{~K}$ up to an applied magnetic field of 5 Tesla (Figure 3.3). The hysteresis loop shows a magnetization saturation of $0.73 \mu_{B} / f . u$. at $4 \mathrm{~K}$ and in $5 \mathrm{~T}$, a $53 \%$ of the bulk value [218]. This decrease in the magnetization can be attributed to the presence of antisites, whose concentration can be estimated from equation 3.3 obtaining an AS presence of $24 \%$. This value is relatively low and comparable with the AS concentration obtained from the XRD measurements, demonstrating the quality of the SCRO target [211].

Once the target was synthesized and completely characterized demonstrating its high stoichiometric and structural quality, it was loaded into the PLD system and the film deposition optimization process was started. 


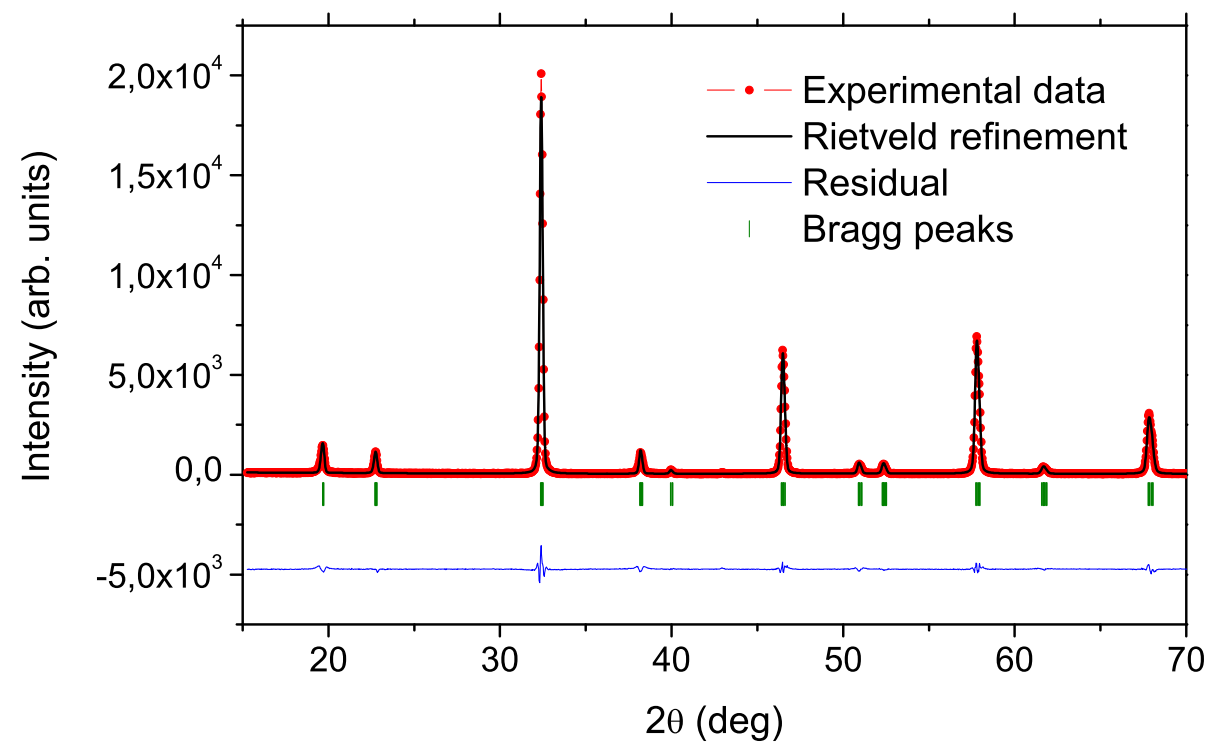

Figure 3.2: XRD $(\theta-2 \theta)$ from the SCRO target. The black line represents the Rietveld method simulation, and the blue line the residual.

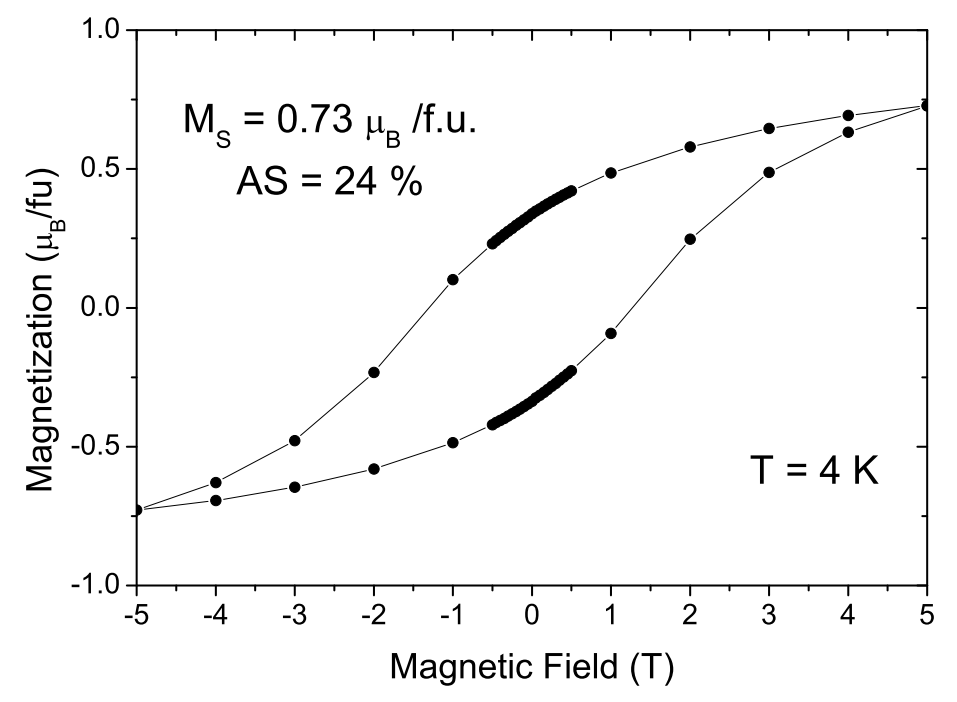

Figure 3.3: Hysteresis loop of an optimized SCRO target at $4 \mathrm{~K}$. 


\subsubsection{A morphological study of the $\mathrm{SrTiO}_{3}(001)$ substrate}

STO (001) substrates were chosen in order to grow epitaxial SCRO thin films due to the small lattice mismatch between the substrate and the film $(<0.1 \%)$.

As it was explained in section 2.5, strontium titanate, $\mathrm{SrTiO}_{3}$ (tausonite) is a perovskite with a Pm3m space group, lattice parameter of $3.905 \AA$ and a density of $5.12 \mathrm{~g} / \mathrm{cm}^{3}$. In a unit cell, Ti ions are located at the cube corner, a $\mathrm{Sr}$ ion in the centre of the cube, and $\mathrm{O}$ ions on the cube edges (see figure 3.4). In the [001] direction the crystal is made up from a stack of alternating $\mathrm{TiO}_{2}$ and $\mathrm{SrO}$ layers, which means that two charge neutral (001) bulk (1x1) terminations are possible: the $\mathrm{TiO}_{2}$ surface and the $\mathrm{SrO}$ surface [227]. In the last years, several studies on $\mathrm{STO}(001)$ report different reconstructions of the (001) surface depending on the preparation performed, being (1x1) the most widely studied.

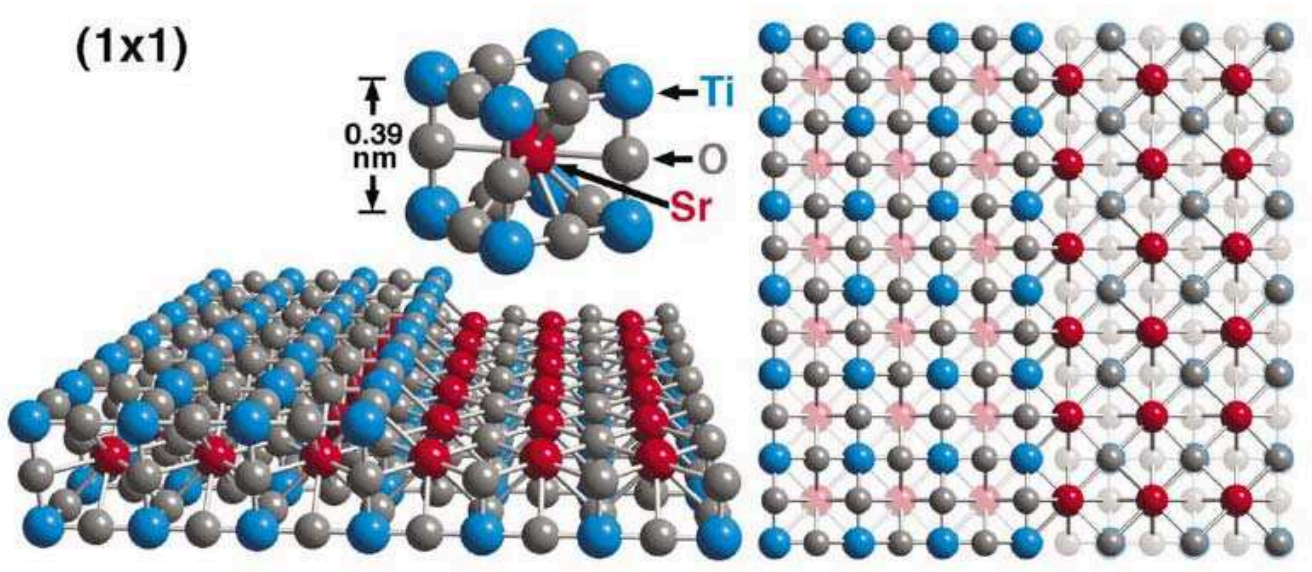

Figure 3.4: The cubic unit cell of $\mathrm{SrTiO}_{3}$ is shown at the top of the figure. The Ti ions (blue) are located at the cube corners, a Sr ion (red) is in the center of the cube, and $\mathrm{O}$ ions (grey) are on the cube edges. A (001) surface with the two possible (1x1) terminations $\left(\mathrm{TiO}_{2}\right.$ on left, $\mathrm{SrO}$ on right) is shown in oblique view (left panel) and in top view (right panel) 227].

Therefore, chemical etching and/or thermal treatments are usually performed on STO substrates in order to obtain the desired surface conditions [228], as its surface chemical composition, structure and morphology may affect the growth of the deposited film. 
Although strontium titanate surface is inert to gas adsorption, after heating above $1000{ }^{\circ} \mathrm{C}$, under reducing conditions or after $\mathrm{Ar}^{+}$bombardment [229, 230], its surface becomes reactive to gas adsorption due to the creation of defects. Surface reconstruction of STO crystals begins at temperatures about $1000{ }^{\circ} \mathrm{C}$ and above, when $\mathrm{Sr}$ atoms shift from the lattice sites to adatom positions on the top of four oxygen atoms of the $\mathrm{TiO}_{2}$ - terminated layers and form different ordered structures on the surface, which can undergo different phase transitions [231].

It has been reported that a weak $(1 \mathrm{x} 1)$ terminated surface can be obtained by cleavage in UHV [22]. A better atomically smooth $\mathrm{TiO}_{2}$ - terminated surface with steps one unit cell in height and flat terraces can be obtained by treating the STO (001) substrate with a $\mathrm{pH}$-controlled $\mathrm{NH}_{4} \mathrm{~F}-\mathrm{HF}$ solution [23], which removes remaining $\mathrm{SrO}$ terminations. After this chemical treatment, the STO surface has carbon contamination that can be completely eliminated in an ultra-high vacuum (UHV) annealing at temperatures higher than $600{ }^{\circ} \mathrm{C}$ [24]. However, annealing at high temperatures in UHV can induce Ti deficiency [25], segregation of Sr atoms on the surface [26 28] and oxygen deficiency [22]. Therefore, the annealing should be carried out in $\mathrm{O}_{2}$ atmosphere in order to prevent the formation of oxygen-deficient surface phases [26].

As a result, we believe that before optimizing the growth conditions of the SCRO films it is necessary a morphological study of the STO (001) substrates, which was performed by means of AFM in tapping mode with a Veeco microscope. The as-received, solvent cleaned, commercial STO substrates present smooth structure-less surfaces (figure 3.5-a). After annealing the STO up to $800{ }^{\circ} \mathrm{C}$ for 30 minutes in air atmosphere, terraces with wavy steps appear (3.5. b) that straighten considerably if the same annealing is performed in oxygen atmosphere (3.5-c).

However, we should note that reproducing the real substrate surface conditions prior to deposition is quite difficult without in-situ characterization techniques. Our approach is to control the conditions in which the substrate reaches the deposition temperature, and use the same parameters in all the samples. Once the substrate surface behavior is studied as a function of the heating atmosphere and final temperature, the SCRO growth deposition optimization can be carried out. 

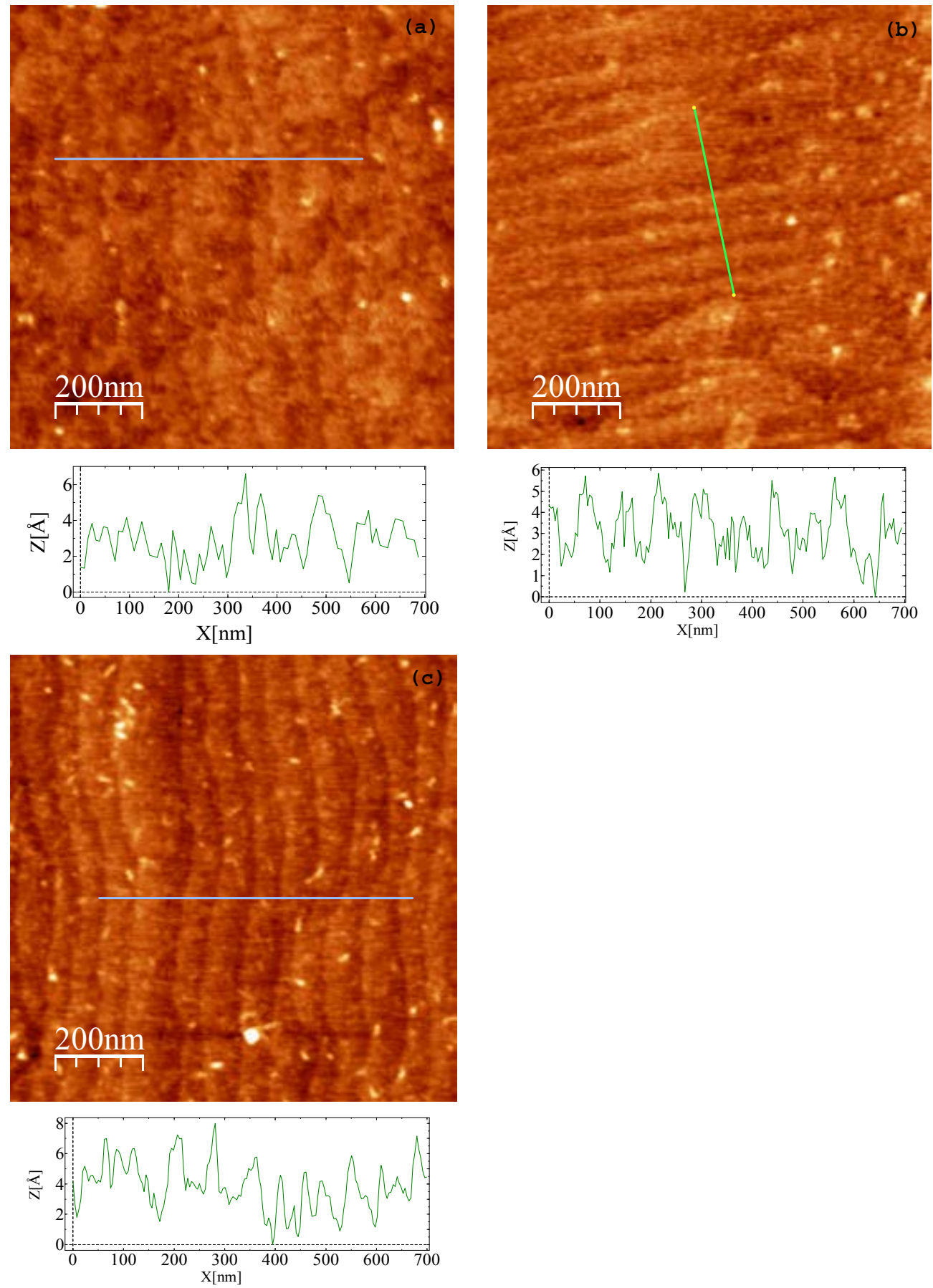

Figure 3.5: $1 \mu m \times 1 \mu m$ AFM images from STO(001) substrates in tapping mode in a Veeco microscope with a $70 \mathrm{kHz}$ tip. The lines define the regions described by the profiles. (a): Clean substrate. (b): Substrate annealed in air at $800{ }^{\circ} \mathrm{C}$ for 30 min. (c): Substrate annealed in oxygen flux at $800{ }^{\circ} \mathrm{C}$ for $30 \mathrm{~min}$. 


\subsubsection{Growth Optimization of $\mathrm{Sr}_{2} \mathrm{CrReO}_{6}$ films.}

We have studied the influence of different growth conditions on the magnetic and structural properties of the films: deposition rate, substrate temperature, and partial oxygen pressure.

The films were deposited by PLD from stoichiometric polycrystalline SCRO targets using a $\mathrm{KrF}$ laser $(248 \mathrm{~nm}), 10 \mathrm{~Hz}$ repetition rate, and laser energy density at the target of $1.5-2.0 \mathrm{~J} / \mathrm{cm}^{2}$. The desired deposition substrate temperature was reached at a rate of $10^{\circ} \mathrm{C} / \mathrm{min}$ and held for 30 minutes in order to obtain the thermic equilibrium in the substrate. Then, the ablation of the target started. After the deposition, the substrate temperature was decreased at $10{ }^{\circ} \mathrm{C} / \mathrm{min}$.

When depositing films in oxygen atmosphere the deposition rate need to be thoroughly controlled, as these two parameters are coupled. Therefore, the growth rate was kept constant at about $4 \times 10^{-3} \mathrm{~nm} /$ pulse. Initially, a large quantity of samples was produced, as both the substrate temperature and oxygen pressure have to be optimized, and films were grown with substrate temperatures varying between $600{ }^{\circ} \mathrm{C}$ and $850^{\circ} \mathrm{C}$ and oxygen partial pressures ranging from $\mathrm{P}\left(\mathrm{O}_{2}\right)=1 \times 10^{-4}$ to $7 \times 10^{-4}$ Torr.

However, the amount of samples needed in order to obtain the optimal deposition conditions can be reduced down to a manageable number by following this procedure: when the partial oxygen pressure $\mathrm{P}\left(\mathrm{O}_{2}\right)$ needs to be studied, the substrate temperature $\mathrm{T}_{S}$ is fixed at a given value (in our case: $800{ }^{\circ} \mathrm{C}$ ), whereas $\mathrm{P}\left(\mathrm{O}_{2}\right)$ was maintained at $2.6 \times 10^{-4}$ Torr when $\mathrm{T}_{S}$ during deposition was varied. The partial oxygen pressure and substrate temperature fixed values were carefully selected. The substrate temperature value at which the partial oxygen pressure was chosen at $800{ }^{\circ} \mathrm{C}$ in agreement with the high temperatures needed for the deposition of other double perovskites. Then, the partial oxygen pressure was fixed at a value for which the films had relatively high quality and the substrate temperature was varied, verifying that the first fixed substrate temperature was acceptable.

In order to study the films structure and roughness, all the samples were characterized by XRD $(\theta-2 \theta, \phi$ scans, and reciprocal space maps) and XRR, and also by VSM magnetometry in order to verify their cationic order and

stoichiometry. STEM experiments were performed only in the films deposited with the optimal growth parameters. 


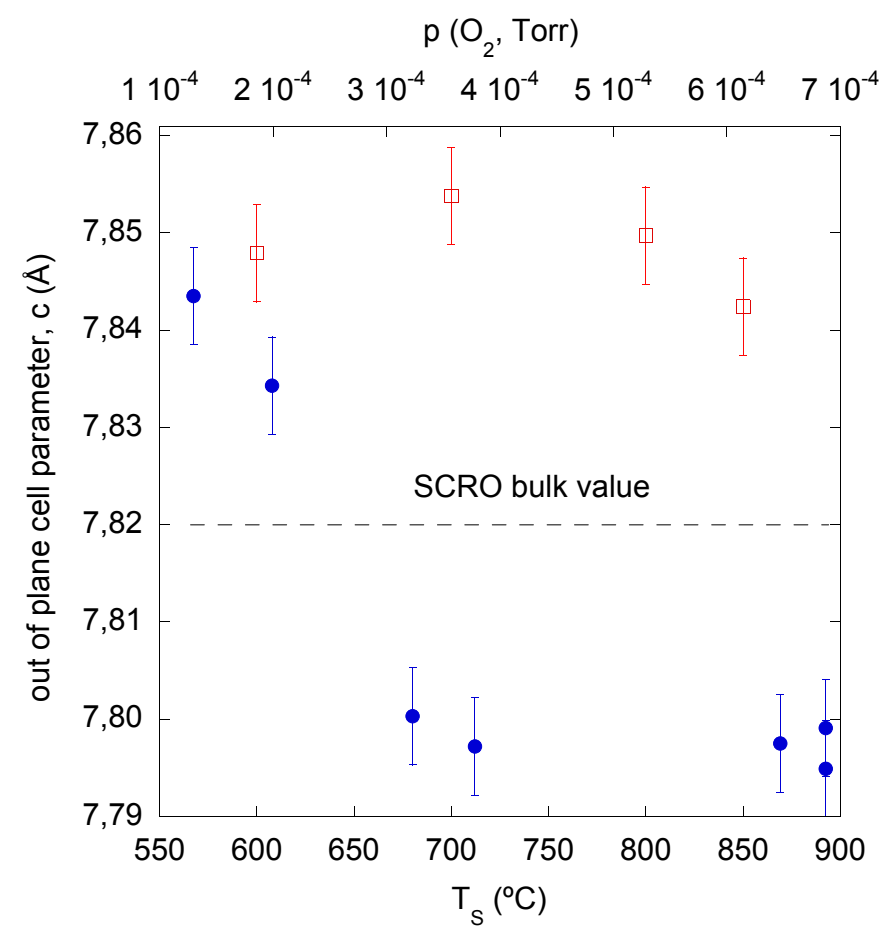

Figure 3.6: Out-of-plane cell parameter, $\mathrm{c}_{\text {out }}$, as a function of both the substrate temperature, $\mathrm{T}_{S}$ (fixing the partial oxygen pressure at $2.6 \times 10^{-4}$ Torr, open red squares) versus the oxygen partial pressure $\mathrm{P}_{\mathrm{O} 2}$ during deposition (at a substrate temperature of $800{ }^{\circ} \mathrm{C}$, block blue circles).

High resolution x-ray diffraction $\theta-2 \theta$ scans near the symmetrical (002) Bragg peak from the STO substrate were performed, showing also the (004) reflection from the SCRO film, and as the out-of-plane cell parameter $\left(\mathrm{c}_{\text {out }}\right)$ can be calculated from the bragg position of the SCRO reflection, a complete study of the oxygen pressure and substrate temperature influence on the film structure was performed (see figure 3.6). It was observed that changing $\mathrm{T}_{S}$ has a small effect on $c_{\text {out }}$, which is always about $0.4 \%$ larger than the SCRO bulk value $\left(\mathrm{a}_{S C R O}=7.82 \AA\right)$, while in contrast, upon increasing $\mathrm{P}\left(\mathrm{O}_{2}\right), \mathrm{c}_{\text {out }}$ diminishes and eventually decreases below the SCRO bulk value.

The magnetic properties dependence with growth conditions of epitaxial SCRO thin films was quantified by saturation magnetization values $\mathrm{M}_{S}$ determined from room-temperature VSM hysteresis loops at room temperature, with the magnetic field applied in the plane of the film (Figure 3.7 and inset). It was observed that $\mathrm{M}_{S}$ shows the highest values $\sim 1 \mu_{B} / f$.u. between 700 and $800^{\circ} \mathrm{C}$, which is higher than that used in sputtered SCRO thin films $\left(527-627^{\circ} \mathrm{C}\right)$ [225] but similar to those utilized in synthesizing epitaxial SCWO epitaxial thin films by PLD [213, 214]. 
However, $\mathrm{M}_{S}$ increases above the SCRO bulk value at high $\mathrm{P}\left(\mathrm{O}_{2}\right)$, which could be related to the segregation of a $\mathrm{CrO}_{x}$ oxide at the surface of the film, in a similar fashion to the reported $\mathrm{FeO}_{x}$ segregation in the case of SFMO films [221].

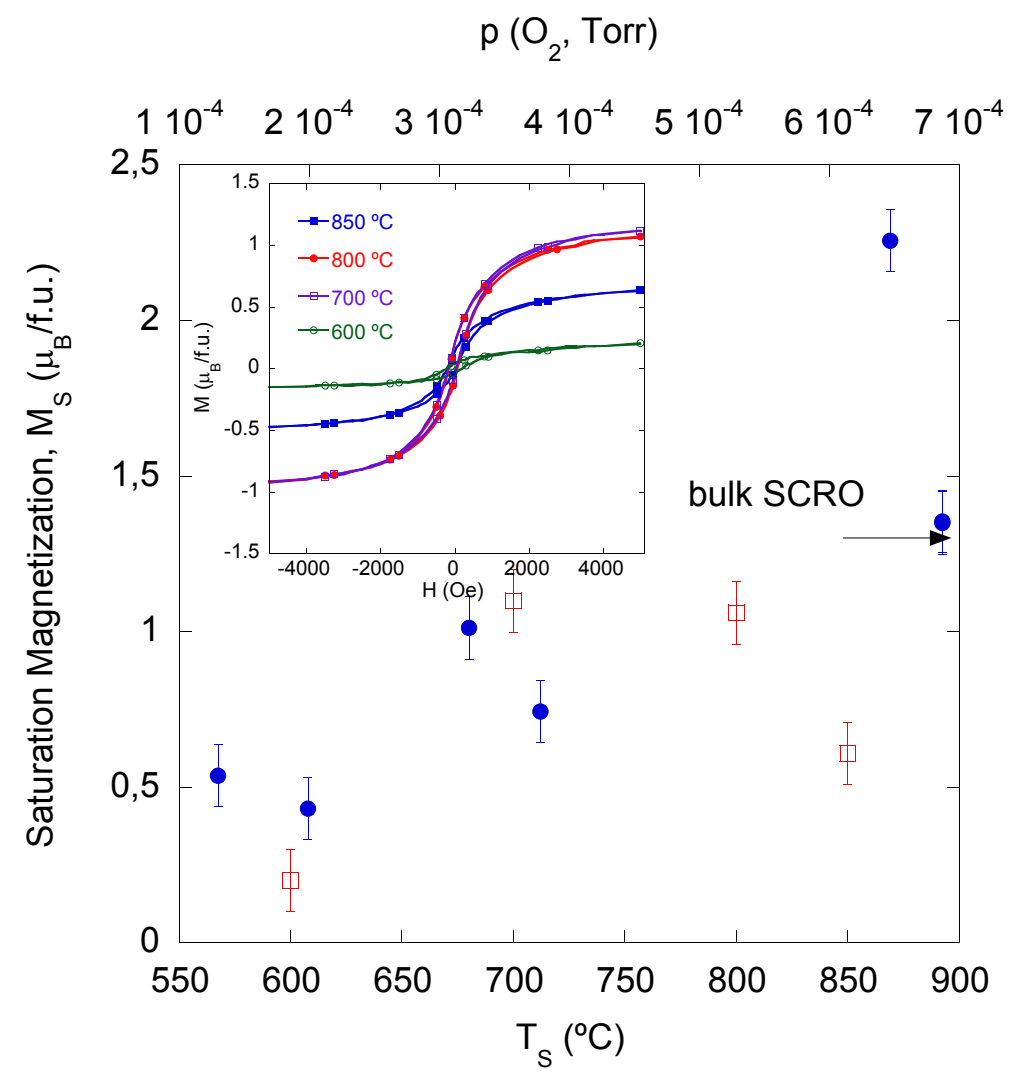

Figure 3.7: Saturation magnetization values, $\mathrm{M}_{S}$, as a function of both the substrate temperature, $\mathrm{T}_{S}$ (fixing the partial oxygen pressure at $2.6 \times 10^{-4}$ Torr, open red squares) versus the oxygen partial pressure $\mathrm{P}\left(\mathrm{O}_{2}\right)$ during deposition (at a substrate temperature of $800{ }^{\circ} \mathrm{C}$, block blue circles).

Therefore, the optimum growth conditions are found at a substrate temperature $\mathrm{T}_{S}=800{ }^{\circ} \mathrm{C}$ and partial oxygen pressure in the range $\mathrm{P}\left(\mathrm{O}_{2}\right)=$ $2.5(5) \times 10^{-4}$ Torr, for a deposition rate of $4 \times 10^{-3} \mathrm{~nm} /$ pulse. 


\subsection{Structural, magnetic and electrical transport char- acterization}

Once the narrow window of growth parameters is found, a deep study of the SCRO films can be performed [232]. In figure 3.8 the symmetrical High Resolution X Ray Diffraction pattern near the (002) Bragg peak from the STO substrate is shown, and the (004) reflection from the SCRO film can be also observed, which gives the expected orientation STO [001] // SCRO [001]. Rocking curves of the 004 peak had a FWHM below $0.03^{\circ}$, which is very close to that of the STO substrate (typically $0.015^{\circ}$ ). In addition, Laue oscillations up to 6 th order are detected around the film diffraction peak indicating that the film grows coherently strained, and their periodicity yields a coherence length $\xi=48.0(5) \mathrm{nm}$.

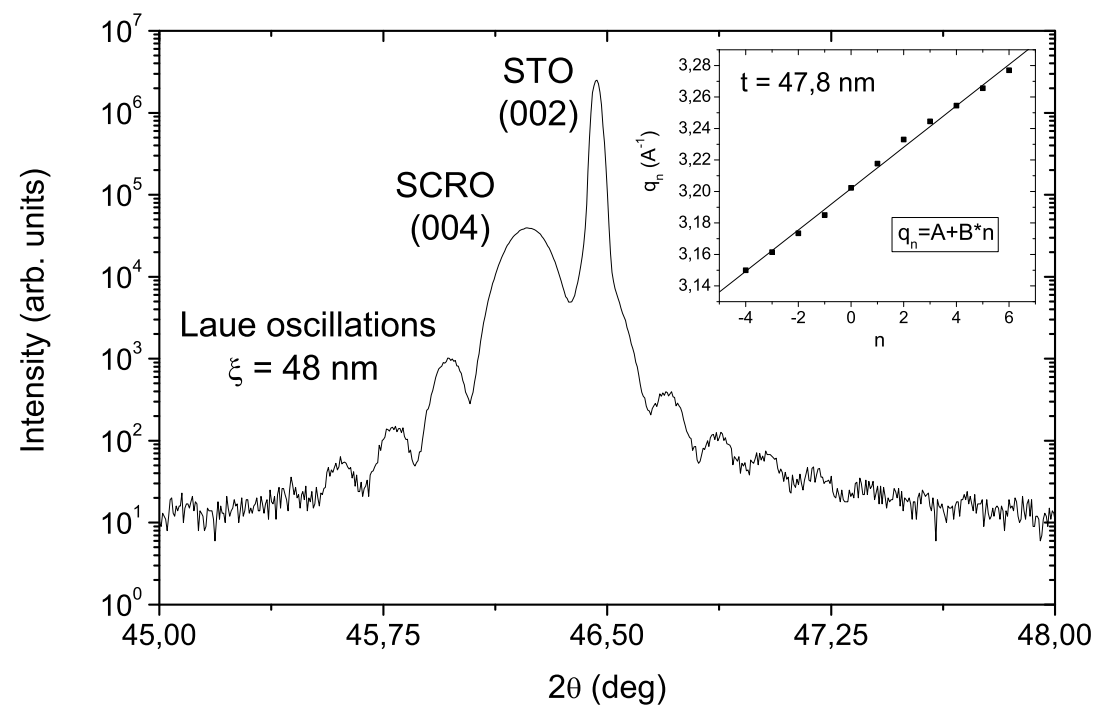

Figure 3.8: XRD measurement $(\theta-2 \theta)$ from a SCRO film grown on STO (001). Inset: Position of the Laue oscillations maximums in q-space. From the linear fit, the coherence length can be obtained, $\xi=2 \pi / \Delta q$.

In order to measure the film thickness independently from its crystalline quality, XRR measurements (figure 3.9) were carried out. From the numerical analysis of the spacing between the Kiessig fringes the film thickness was found to be $55.6 \pm 0.1 \mathrm{~nm}$, which compares reasonably well with the coherence length obtained from the Laue oscillations, indicating the existence of crystalline coherence essentially along the full thickness of the film. 
From the numerical fit of the XRR data the roughness $(\sigma)$ of the double perovskite film and the interface of the film with the substrate, and also the SCRO density are obtained (table 3.1). The film density is close to the bulk value, and its roughness is relatively low. However, the measured substrate roughness is higher than its value previous to the SCRO deposition, indicating a possible surface degradation of the STO surface during the growth process which should be taken into account when integrating the STO // SCRO bilayer in a more complex heterostructure.

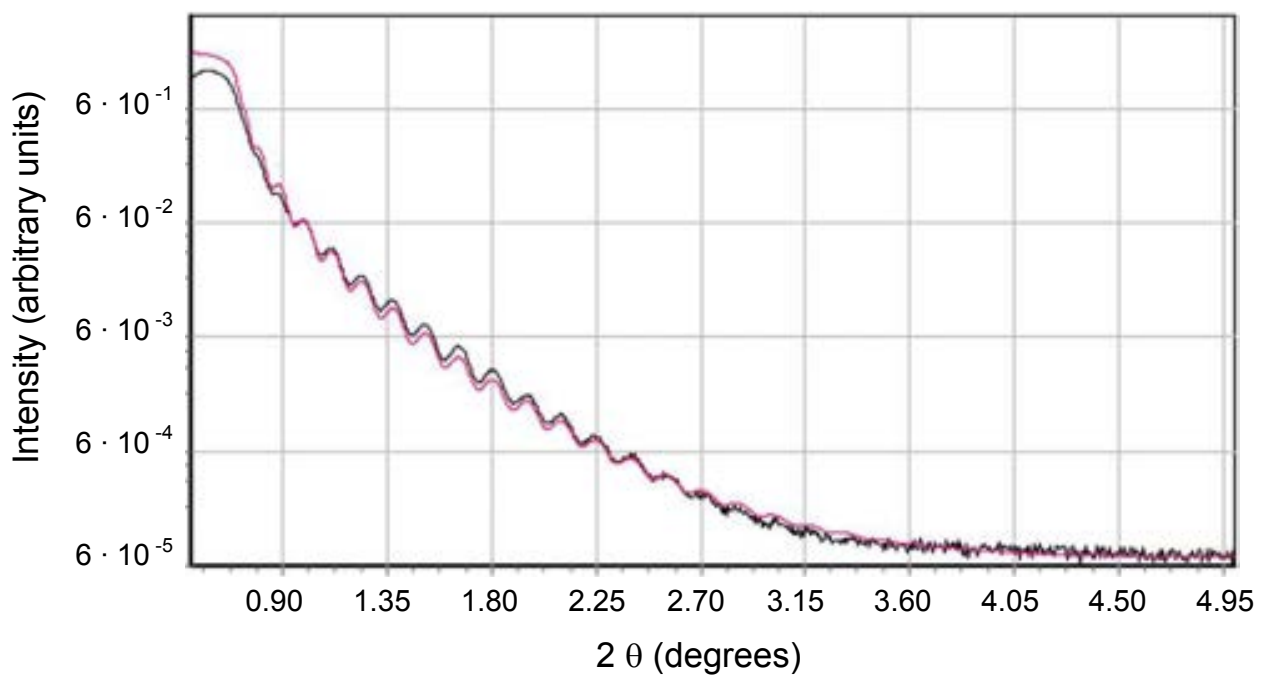

Figure 3.9: XRR measurement from a $55.6 \mathrm{~nm}$ thick sample produced at the optimal growth parameters. The red line corresponds to the numerical fit.

\begin{tabular}{||c|c|c|c||}
\hline \hline Material & Thickness $(\mathrm{nm})$ & Roughness $(\mathrm{nm})$ & Density $\left(\mathrm{g} / \mathrm{cm}^{3}\right)$ \\
\hline $\mathrm{Sr}_{2} \mathrm{CrReO}_{6}$ & 55.54 & 0.58 & 6.600 \\
\hline $\mathrm{SrTiO}_{3}$ & Substrate & 0.95 & 5.117 \\
\hline \hline
\end{tabular}

Table 3.1: Values obtained from the XRR numerical fit in figure 3.9.

The SCRO out-of-plane lattice parameter can be extracted from the peak position in the $\theta-2 \theta$ measurement obtaining:

$$
c_{\text {out }}=7.85 \AA
$$

This value is slightly larger $(0.4 \%)$ than the bulk SCRO lattice parameter $\left(a_{b u l k}=7.82 \AA\right)$, but in good agreement with that found in sputtered thin films [225]. 
RSM performed near the asymmetrical reflection (013) from the STO shows the (116) reflection from the SCRO (figure 3.10), indicating the epitaxial growth of the SCRO film on the STO substrate. As the scattering vector is inversely proportional to the lattice parameters, both in- and out-of-plane lattice parameters can be extracted from the RSM, obtaining the in-plane orientation of the SCRO film:

$$
c_{\text {out }}=7.85 \dot{A} \quad a_{\text {in }}=5.52 \dot{A}
$$

Considering that $2 \cdot a_{S T O}=a_{i n} \sqrt{2}$, it is demonstrated that the film is fully strained, and the epitaxy relationship is thus STO (001) [110] // SCRO (001) [100].

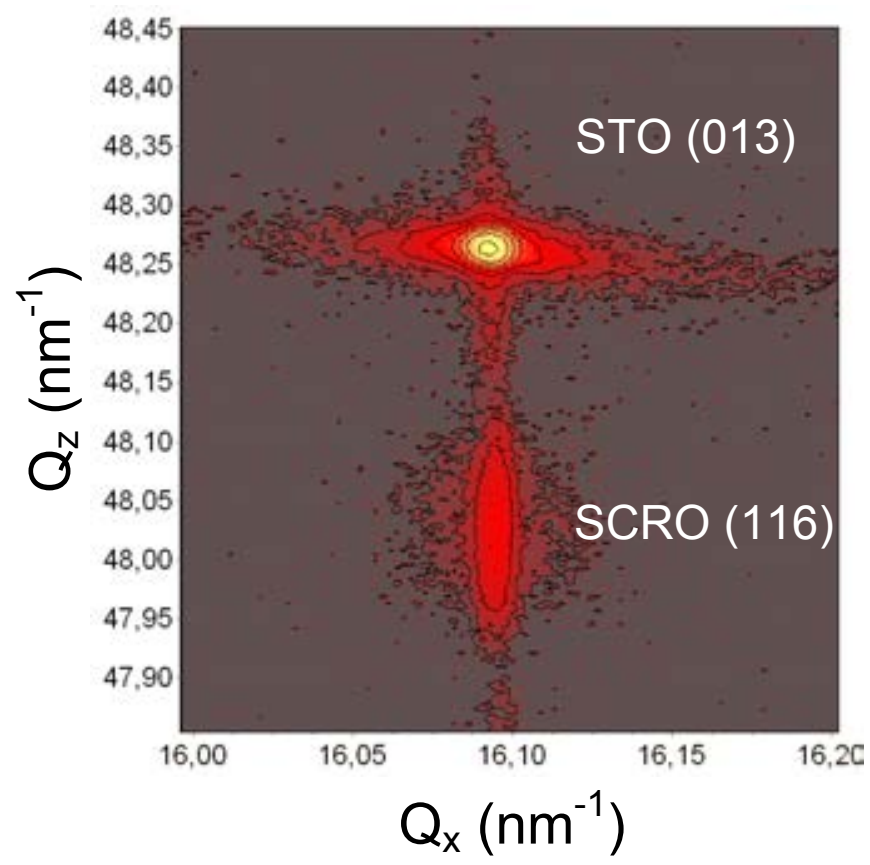

Figure 3.10: RSM in the vicinity of the asymmetrical SCRO (116) and STO (013) reflections. $\mathrm{Q}_{x}$ and $\mathrm{Q}_{z}$ are along the $\mathrm{STO}[010]$ and [001] directions, respectively.

Once the crystalline quality of the films is achieved, the existence of cationic B-site ordering should be confirmed. This can be observed by looking for the (011) superlattice peak, which is forbidden in terms of crystal symmetry and therefore, appears only when the cationic order is maintained. In figure 3.11, a $\phi$ - scan of the asymmetrical (011) reflection from the SCRO film is shown. As expected, a clear fourfold symmetry is observed, demonstrating both the epitaxial growth and the existence of cationic ordering. 


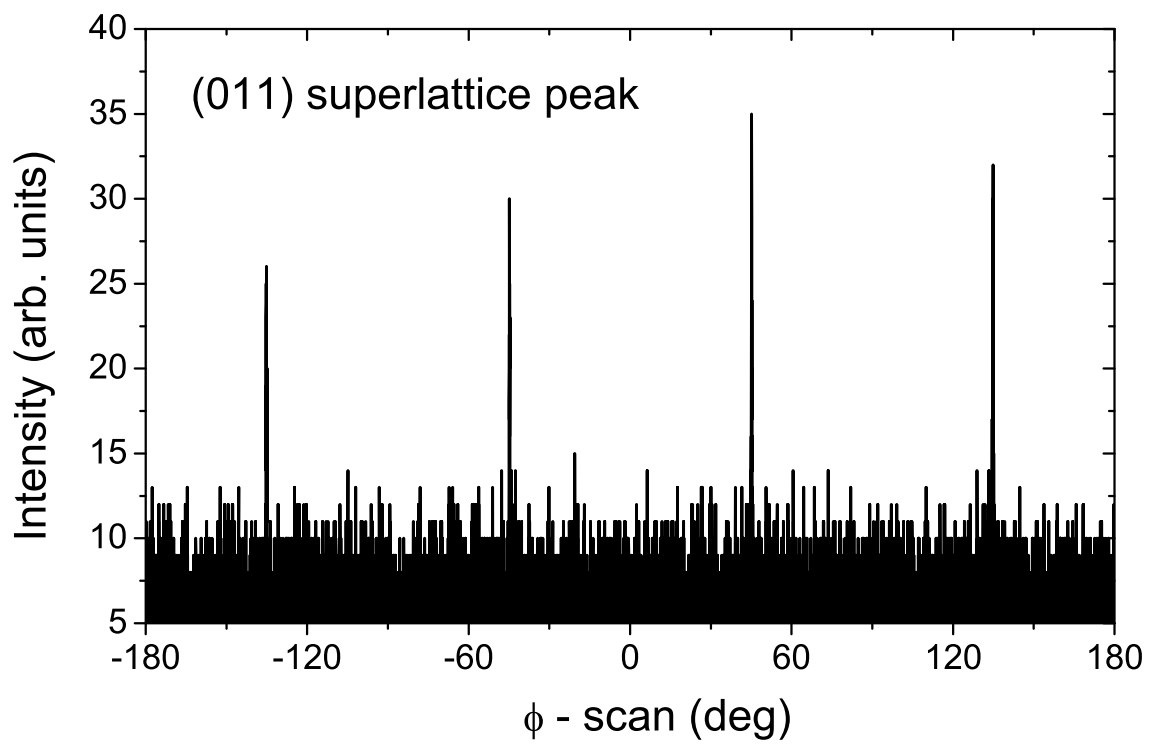

Figure 3.11: $\phi-$ scan of the asymmetrical (011) superlattice reflection from the SCRO film. $\phi=0$ corresponds to the [101] direction of the STO substrate.

Once the structural quality and the cationic ordering of the SCRO films was confirmed, a magnetic and transport characterization need to be performed.

Magnetization measurements as a function of the applied magnetic field at room temperature with the field applied in the plane of the film surface and with a maximum value of 1.9 Tesla were performed (figure 3.12). From the hysteresis loop we obtained a value of $1.1 \mu_{B} / f$.u. for the saturation magnetization, which is about $20 \%$ less than the value reported for bulk SCRO (1.38 $\left.\mu_{B} / f . u.\right)$. As it has been pointed out in section 3.1, this effect is generally explained due to the presence of AS, whose concentration (w) can be calculated from the saturation magnetization value of our samples and equation 3.3 , obtaining an AS concentration: $w=10 \%$.

The coercive field in all the SCRO thin films has been found to be small, $\mathrm{H}_{C} \sim 120$ Oe, whereas bulk SCRO has a coercive field of $3.1 \mathrm{kOe}$ at $300 \mathrm{~K}$ [233]. Asano et al. reported a significant change in coercivity by increasing the substrate temperature by $100{ }^{\circ} \mathrm{C}$ [225]. Therefore, an increase in the coercive field would be expected with further annealing. However, it could also modify the samples surface morphology, producing the segregation of parasitic phases. 


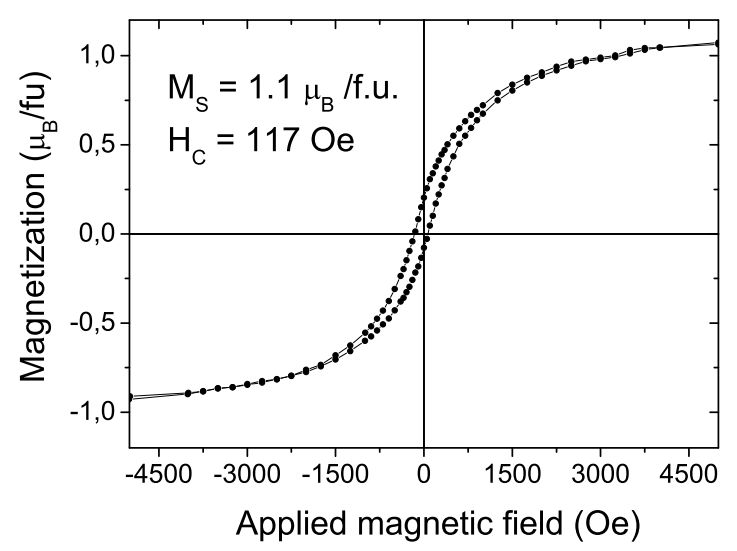

Figure 3.12: Magnetization measurement as a function of magnetic field at room temperature (300K).

The order temperature of the SCRO films was obtained by measuring the magnetization as a function of temperature by means of VSM, in an applied magnetic field of $3 \mathrm{kOe}$ (see figure 3.13) in the plane of the film. The Curie temperature was found to be $\mathrm{T}_{C}=481(2) \mathrm{K}$, which is about $22 \%$ smaller than that reported for bulk SCRO [204] or in sputtered films [225]. This decrease in $\mathrm{T}_{C}$ can probably due to a higher $\mathrm{AS}$ concentration or a non-perfect oxygen stoichiometry.

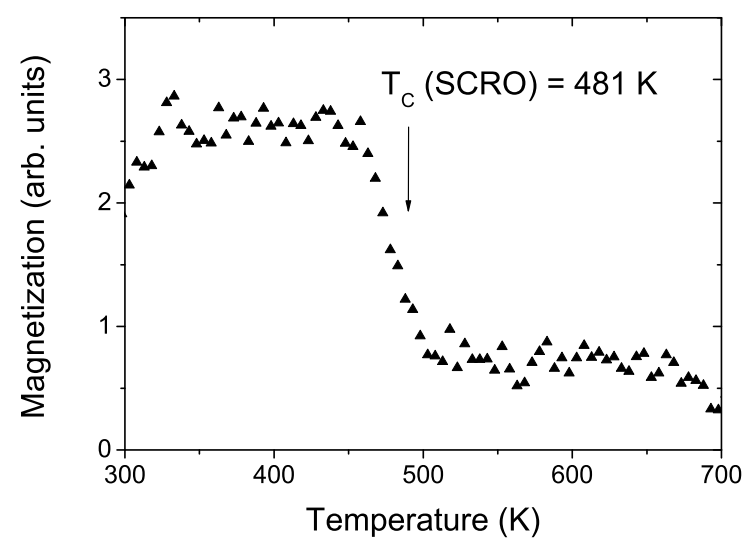

Figure 3.13: Magnetization as a function of temperature in an applied magnetic field of $3 \mathrm{kOe}$. 
The electrical resistivity, $\rho$, was measured as a function of temperature in a selected optimized film in the Van-der-Pauw geometry, finding a room temperature resistivity of around $2.8 \mathrm{~m} \Omega \cdot \mathrm{cm}$ (figure 3.14). This value is smaller than bulk polycrystalline SCRO [204], but in good agreement in both value and temperature dependence with optimized sputter deposited SCRO thin films [225].

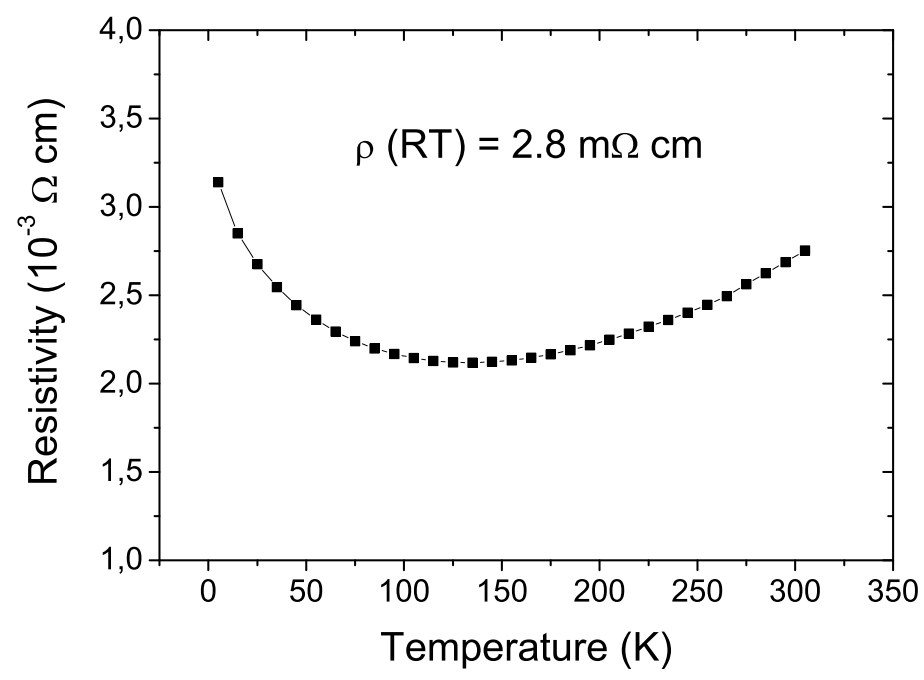

Figure 3.14: Temperature dependence of the electrical resistivity $(\rho)$ for a selected optimized SCRO thin film. 


\subsection{Transmission Electron Microscopy}

As the presence of AS has a direct influence in the structural and magnetic properties of the SCRO, a deep study of the cationic order has been performed by HR TEM. With this purpose, SCRO films were deposited on STO substrates with two different crystallographic orientations, (001) and (111), and investigated by means of Scanning Transmission Electron Microscopy (STEM).

\subsection{1 $\mathrm{STO}(001) / / \mathrm{SCRO}$}

STEM observations of SCRO films grown with the optimized deposition conditions on STO (001) substrates were performed in a VG Microscope HB501UX STEM operated at $100 \mathrm{keV}$ and equipped with a Nion aberration corrector and a Gatan Enfina parallel electron energy loss spectrometer (PEELS), in the Oak Ridge National Laboratory (USA).

The optimized SCRO films are flat and with uniform thickness over long lateral distances, as it is observed in the low magnification Z-contrast image of a cross section STEM specimen prepared by mechanical thinning and Ar ion milling (figure 3.15).

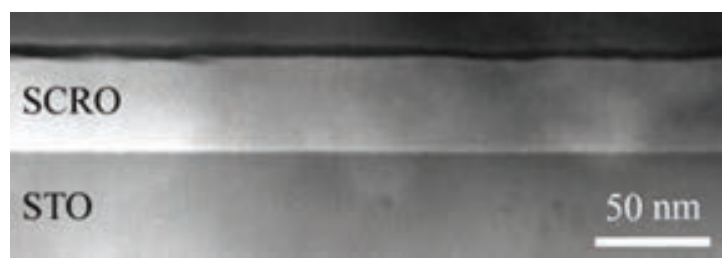

Figure 3.15: Low magnification cross-section STEM image of a SCRO film grown on STO(001). Performed by Dr. C. Magen in the Oak Ridge National Laboratory (USA)

The high resolution Z-contrast STEM image near the interface region between the SCRO film and the STO substrate displayed in figure 3.16 evidences the epitaxial growth of the SCRO with a pseudocubic crystal structure coherent with the perovskite structure of the substrate, as brighter columns corresponding to the higher average atomic number of the SCRO film (i.e. $\mathrm{Cr} / \mathrm{Re}$ atoms) grow on top of the darker $\mathrm{Ti}$ columns of the substrate, both corresponding to the $\mathrm{B}$ position of the perovskite structure.

The epitaxy relations are, therefore, STO (001) [110] // SCRO (001) [100] as proposed from the x-ray structural characterization, and no clear sign of stress relaxation is detected, which agrees with the small lattice mismatch expected and with the in-plane lattice parameter obtained from the Reciprocal Space Maps (Fig. 3.10 in section 3.3). 
In addition, it should be pointed out that the interface is sharp and coherent and no secondary phases were observed, which is relevant if the SCRO film is to be used as an active electrode in a spintronic device.

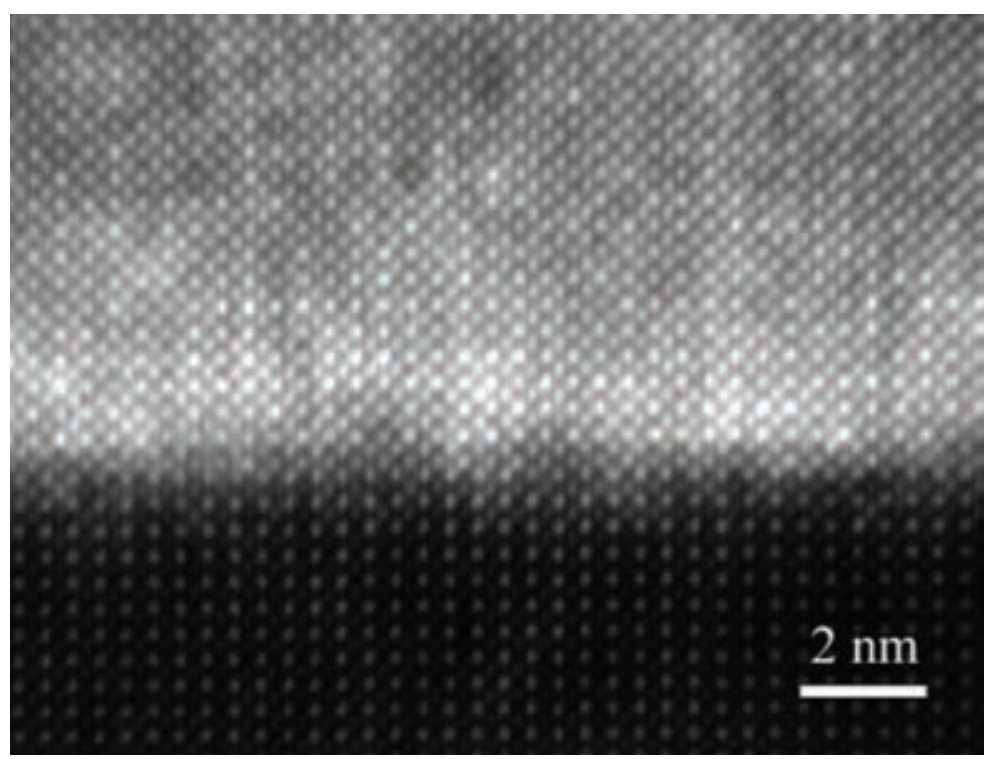

Figure 3.16: High resolution STEM image near the interface region of the SCRO film with the STO(111) substrate. Performed by Dr. C. Magen in the Oak Ridge National Laboratory (USA).

Regarding the SCRO films stability, the same STO(001) // SCRO film has been measured in a high resolution Titan Transmission Electron Microscope in the INA (Zaragoza, Spain), one year after Figure 3.16 was obtained. Figure 3.17 shows the high resolution Z-contrast aberration corrected ADF-STEM image, demonstrating that, one year later, the cationic ordering and the sharp interface of the SCRO film with the STO substrate is maintained, and therefore, the SCRO double perovskite thin films are structurally and chemically stable. 


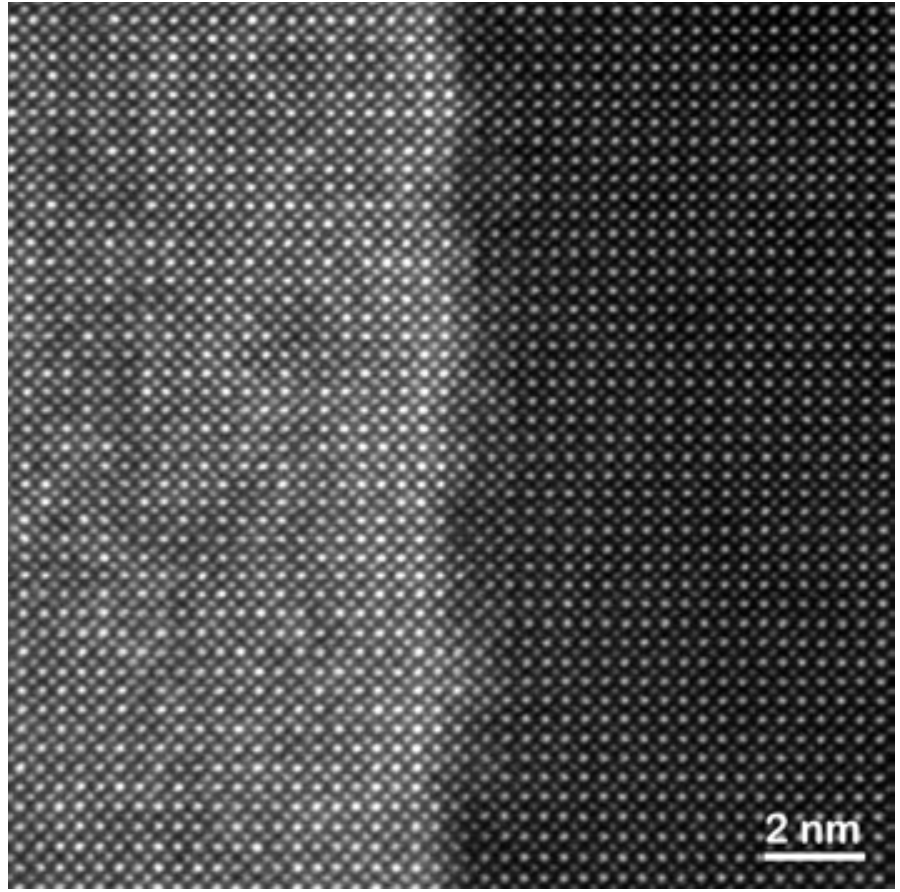

Figure 3.17: Measurement performed by Dr. C. Magen in the INA in Zaragoza (Spain). High resolution ADF-STEM image of the SCRO film interface with the STO substrate.

\subsubsection{STO(111) // SCRO: cation ordering}

The average cationic ordering has indirectly been deduced from x-ray Diffraction and magnetization measurements in section 3.3. However, the direct visualization of cationic order with atomic resolution in real space could be performed by means of STEM, which would provide a better insight on the interplay between the local structure and chemical disorder.

The imaging of the superstructure in a double perovskite can only be achieved by orienting the sample along a direction where the expected structure is adequately projected, and SCRO thin films grown on STO (111) substrates have the superstructure direction more easily accessible. Therefore, in order to perform STEM measurements of the double perovskite thin films, $\sim 40 \mathrm{~nm}$ thick SCRO films were grown on STO (111) substrates by using the previously optimized deposition conditions.

The samples were characterized by means of XRR, XRD and VSM experiments, demonstrating their crystalline, structural and stoichiometric quality, and therefore, that the same deposition conditions are suitable for the SCRO growth on both STO (001) and STO (111) substrates. 
The samples thickness and roughness were obtained from XRR measurements, obtaining similar roughnesses as in the SCRO films grown on STO (001), and XRD $\theta-2 \theta$ experiments (figure 3.18) showed the absence of parasitic phases and the $\operatorname{SCRO}(011)$ growth orientation on the $\operatorname{STO}(111)$. The presence of the $\mathrm{SCRO}(011)$ superlattice peak was observed, evidencing the existence of cationic ordering in the double perovskite thin film. The sample holder in the XRD equipment is made of $\mathrm{Al}$, which explains the $\mathrm{Al}$ (hkl) reflections present at the XRD pattern.

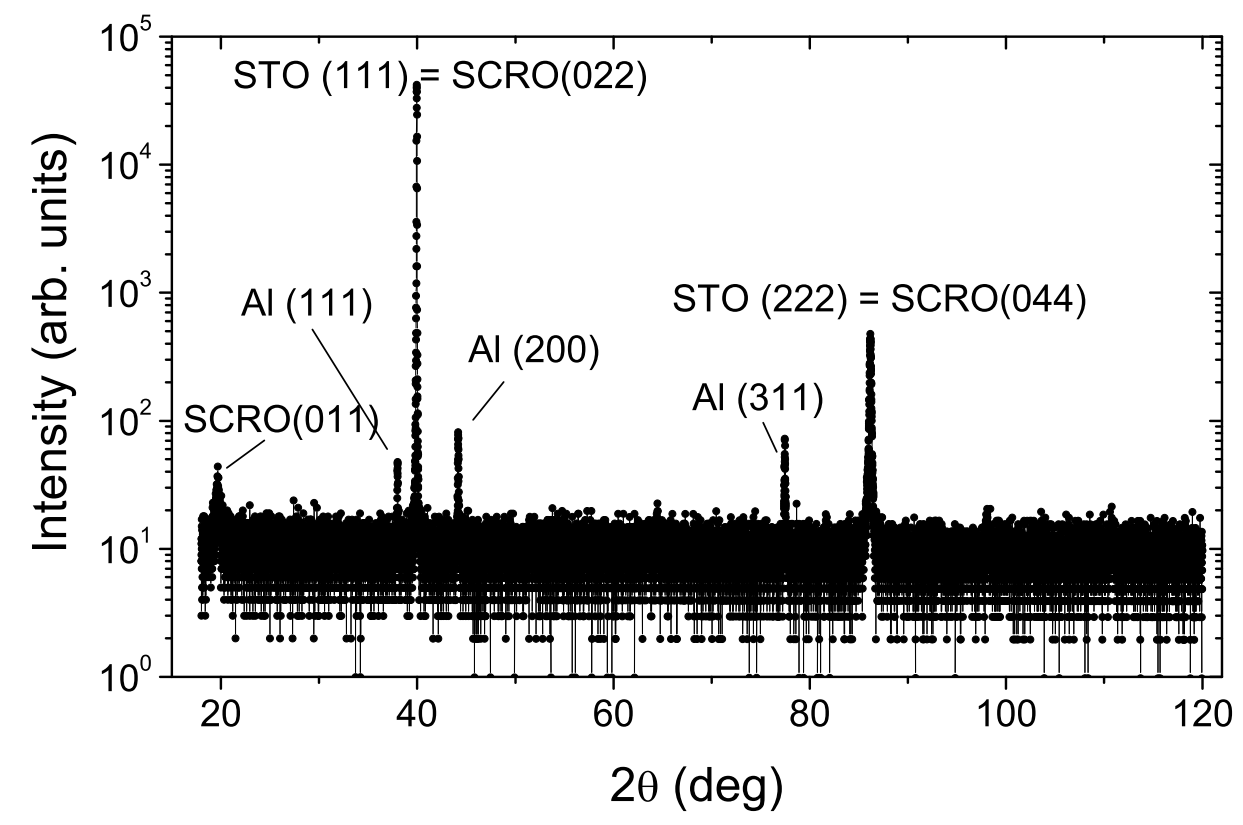

Figure 3.18: X Ray $\theta-2 \theta$ measurement from a STO(111) // ( 40nm) SCRO.

Magnetization measurements as a function of the applied magnetic field were performed by means of VSM at room temperature with the field applied in the plane of the film surface and with a maximum value of 1.9 Tesla (figure 3.19). From the hysteresis loop we obtained a value of $1.2 \mu_{B} / f$.u. for the saturation magnetization, which is about $13 \%$ less than the value reported for bulk $\mathrm{SCRO}\left(1.38 \mu_{B} / f . u\right.$. $)$. Therefore, the AS concentration is $w_{S T O(111) / / S C R O}$ $=6.5 \%$, lower than the value obtained for the SCRO films grown on STO (001) substrates $\left(w_{S T O}(001) / / S C R O=10 \%\right.$, see section 3.3). The coercive field is found to be $\mathrm{H}_{C} \sim 145$ Oe, slightly higher than the value obtained for the SCRO films grown on $\mathrm{STO}(001)$ substrates $\left(\mathrm{H}_{C}=120 \mathrm{Oe}\right)$, but still lower than the bulk value $(3.1 \mathrm{kOe})$. 


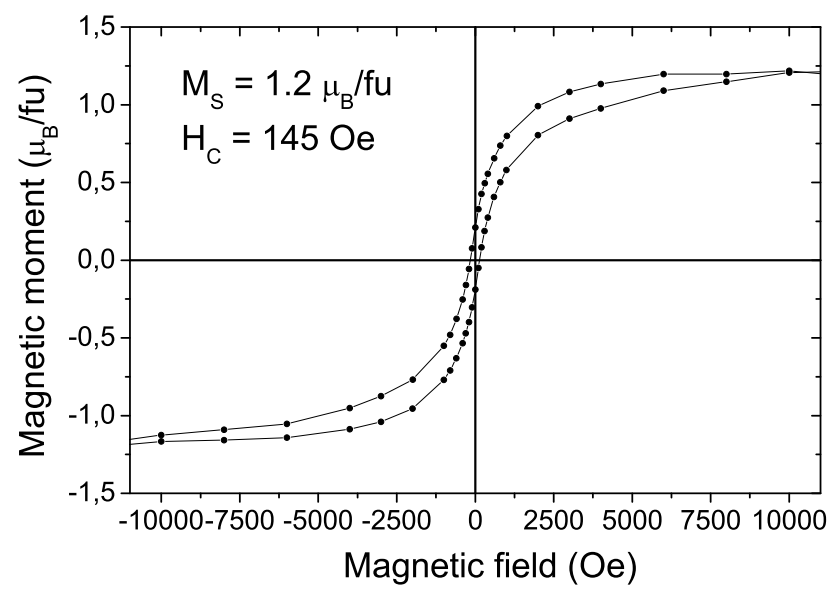

Figure 3.19: Magnetization measurement as a function of magnetic field at room temperature $(300 \mathrm{~K})$ of a $\sim 40 \mathrm{~nm} \mathrm{STO}(111) / /$ SCRO.

The observation of cationic ordering of the B sites and the microstructure of $\mathrm{Sr}_{2} \mathrm{CrReO}_{6}$ thin films was performed in the VG Microscope HB603U STEM equipped with a Nion aberration corrector and operated at an acceleration voltage of $300 \mathrm{keV}$, in the Oak Ridge National Laboratory (USA), and the cross section specimens were prepared by conventional mechanical thinning and ion milling.

Z-contrast aberration corrected annular dark field (ADF) STEM images provide the necessary spatial resolution and chemical contrast to identify the atomic columns corresponding to the different metallic elements of the SCRO structure, visualize the spatial ordering of the $\mathrm{Cr}$ and Re atoms and estimate the degree of cationic order. The B superstructure becomes evident in Fig. 3.20, which focuses on an area with a single twin with uniform crystal structure and shows clearly the formation of a superstructure corresponding to the spatial ordering of the $\mathrm{Cr}$ and Re cations in the B sites.

The inset embedded in Fig. 3.20(a) depicts the multislice image simulation of the ADF STEM image, which shows a good match to the experiment. This agreement is better demonstrated by comparing the intensity profiles of the image (red arrow) and the simulation along the [01ㅣ direction illustrated in Fig. 3.20(b) and (c). The oxygen atoms are not observed due to the very low atomic number $\left(\mathrm{Z}_{O}=8\right)$, the high intensity peaks of the simulation correspond to the Re columns with the largest atomic number $\left(\mathrm{Z}_{R e}=75\right)$, whereas the intermediate intensity is located in the $\mathrm{Sr}$ positions $\left(\mathrm{Z}_{S r}=38\right)$ and the low intensity peaks are related to the $\mathrm{Cr}$ atoms $\left(\mathrm{Z}_{C r}=24\right)$. 
As the contrast between the three types of atomic columns is nicely uniform along a distance of several nanometers, it can be assumed that the concentration of AS is relatively low, in agreement with the concentration obtained from magnetization measurements.
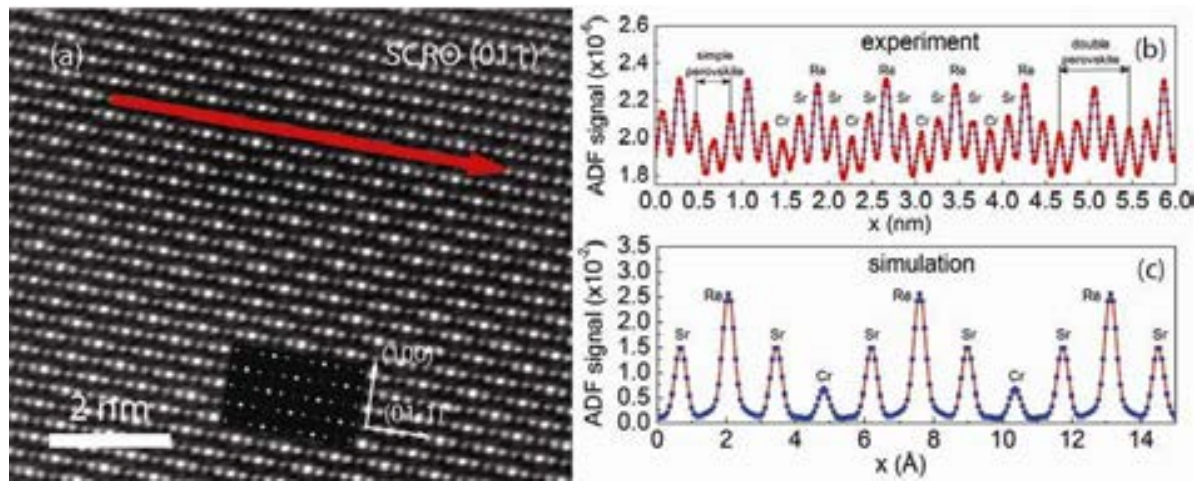

Figure 3.20: Measurements performed by Dr. C. Magen in the Oak Ridge National Laboratory (USA). (a) ADF-STEM image of a single domain of SCRO thin film grown on STO(111) oriented along the pseudocubic zone axis (011) acquired at an acceleration voltage of $300 \mathrm{kV}$. The image has been noise filtered. The inset shows a multislice simulation of the image for a thickness of 20nm. (b) ADF signal profile of the image (a) along the (011) direction (red arrow). (c) ADF intensity profile of the multislice simulation along the same direction.

In conclusion, we have succeeded in the synthesis of epitaxial $\mathrm{Sr}_{2} \mathrm{CrReO}_{6}$ double perovkite thin films, obtaining $\approx 50 \mathrm{~nm}$-thick samples with high crystallinity and relatively large cationic order in a narrow window of growth parameters: substrate temperature, $\mathrm{T}_{S}=750 \pm 50{ }^{\circ} \mathrm{C}$ and in a partial oxygen pressure $\mathrm{P}\left(\mathrm{O}_{2}\right)=2.5(5) \times 10^{-4}$ Torr at a growth rate about $4 \times 10^{-3} \mathrm{~nm} /$ pulse.

The epitaxy relationship has been found to be STO (001) [110] // SCRO (001) [100] by means of HRXRD and STEM microscopy. We have also evaluated the magnetic properties and electrical resistivity of optimized films obtaining typical values of saturation magnetization of $\mathrm{M}_{S}(300 \mathrm{~K})=1 \mu_{B} / f . u$., room temperature resistivity of $\rho(300 \mathrm{~K})=2.8 \mathrm{~m} \Omega \cdot \mathrm{cm}$, and Curie temperature of $\mathrm{T}_{C}=481 \mathrm{~K}$, in good agreement with previous published results in sputtered epitaxial thin films. The AS concentration in our thin films has been estimated from the magnetization measurements, and found to be of the order of $10 \%$.

Finally, STO(001)//SCRO and STO(111)//SCRO films have been measured by means of STEM, evidencing the sharp interface of the SCRO films with the STO substrates, the high cationic order, and the structural and chemical stability of the samples. 



\section{Chapter 4}

\section{FePt epitaxial thin films}

The growth of epitaxial FePt $\mathrm{L}_{0}$-ordered alloy films was carried out by $\mathrm{RF}$ sputtering under the supervision of the scientific coordinator of the magnetic materials research department Dr. Franca Albertini at the IMEM-CNR at Parma (Italy), during a stay of two months. This research group has experience in the preparation and magnetic and structural characterization of FePt thin films with high crystalline quality [234 237].

The RF sputtering equipment available at IMEM (already detailed in section 1.1.2, chapter 1) is specially effective in the growth of epitaxial metallic alloy thin films. Fully operative AFM, MFM (see section 1.2, chapter 11) and AGFM equipments are also available in this research center, allowing an exhaustive magnetic and morphological characterization of the samples prepared. Film characterization was completed by XRD experiments performed in the Bruker x-ray diffractometer available at the INA in Zaragoza (Spain).

\subsection{Introduction}

\subsubsection{FePt: a solution to the superparamagnetic limit}

The nowadays high-density magnetic recording technology is advancing at such a rate that magnetic grains with only a few nanometers in diameter will be required in the next years. However, decreasing the magnetic grain sizes results in an increase of both the Signal to Noise Ratio (SNR) and the thermal instability, which leads to a size limit in technology evolution. 
The thermal instability produce the loss of the stored data quite easily due to the fluctuation of magnetization, and dictates the lower limit to the size of magnetic grains in the recording medium [238]. Therefore, the materials should have enough magnetocrystalline anisotropy energy to overcome the thermal fluctuation energy [238], thus:

$$
K_{u} V=N k_{B} T
$$

where $K_{u}$ is the uniaxial magnetocrystalline anisotropy, $\mathrm{V}$ is the particle volume, $k_{B}$ is the Boltzmann's constant, $T$ is the temperature and $\mathrm{N}$ takes values between 50 and 70 .

Initially, perpendicular recording geometry represents the best answer to the superparamagnetic limit, as it exhibits a potentially higher density than longitudinal recording; for example, for an $\mathrm{SNR}=20 \mathrm{~dB}$, the limiting densities are approximately 500 and $100 \mathrm{Gbit} / \mathrm{in}^{2}$, respectively [239]. However, there are some requirements that should be accounted for when growing thin films for their implementation in perpendicular recording media, that have been reported to be [240]:

- Moderate coercivity (of the order of $10 \mathrm{kOe}$ for perpendicular recording [238]), as it should be high enough to fully exploit perpendicular recording and cannot exceed the write field capability of the head.

- There should be perfect remanence, or a unity squareness ratio.

- Small grain size with a narrow size distribution to reduce the transition noise and to support large recording densities.

- Small exchange interaction between grains, which enables each grain to respond to applied magnetic fields independently, allowing in turn high coercivity and low SNR.

- Small spacing between the soft underlayer and the media to minimize the effective spacing loss.

FePt ferromagnetic thin films have developed great interest in the last years, due to the fact that when this alloy is $\mathrm{L} 1_{0^{-}}$ordered, it presents a high magnetocrystalline anisotropy energy density $\left(7 \times 10^{7} \mathrm{erg} / \mathrm{cm}^{3}[241]\right)$ with the easy magnetization direction perpendicular to the film surface plane ((001)oriented), which solves the superparamagnetic limit problem. Indeed, the theoretical superparamagnetic radius for $\mathrm{FePt}$ is found at a value as low as 3 $\AA$ [238]. In addition, this material has shown high tunnel magnetoresistance when being part of a MTJ with $\mathrm{MgO}(001)$ barrier [46, 50, 51], increasing its interest for spintronics applications. 


\subsubsection{The FePt alloy}

FePt bulk alloy can adopt two different crystal structures for Pt compositions between 40 and 60 at\% $\mathrm{Pt}: \mathrm{L} 1_{0}$ or $\mathrm{A} 1$ phases, as indicated in the bulk $\mathrm{FePt}$ phase diagram (Figure 4.1).

In the $\mathrm{L} 1_{0}$ phase the iron and platinum atoms occupy alternating layers along the [001] axis (FePt chemically ordered state), forming a thermodynamically stable superlattice structure, while the $A 1$ phase has both elements randomly distributed across the layers (chemically disordered state).

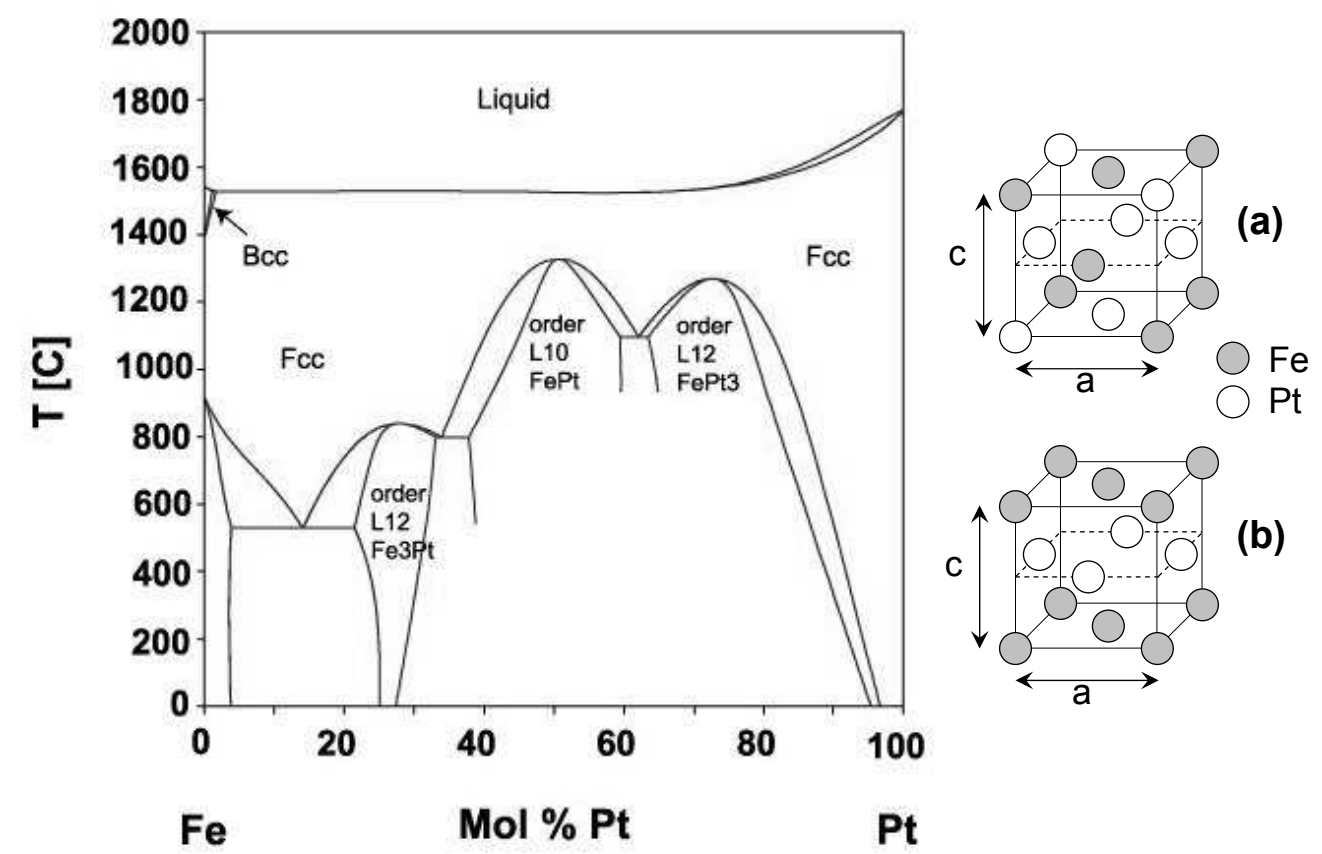

Figure 4.1: Left panel: FePt phase diagram 242]. Right panel: The two crystal phases of FePt. A1 phase (a), also called face centered cubic phase ( $\mathrm{fcc}$ ), a $=\mathrm{c}$ and the $\mathrm{L} 1_{0}$ phase (b), also called face centered tetragonal phase (fct), a $>\mathrm{c}$.

The $\mathrm{L}_{0}$ phase, with the tetragonal spacial group $\mathrm{P} 4 / \mathrm{mmm}$, exhibits high, uniaxial magnetocrystalline anisotropy, with an anisotropy constant $K$ of $7 \times$ $10^{7} \mathrm{erg} / \mathrm{cm}^{3}$, and a saturation magnetization $M_{S}$ of $1140 \mathrm{emu} / \mathrm{cm}^{3}$ with the easy magnetization axis in the c-axis of the tetragonal structure [241]. The lattice parameter along the c-axis is shorter than the one along the a-axis:

$$
\mathrm{a}=3.85 \AA \quad \mathrm{c}=3.71 \AA
$$

The FePt phase diagram is modified when depositing FePt films due to the non-equilibrium conditions of the process. 
Different fabrication methods have been employed to optimize the microstructure and magnetic properties of $\mathrm{L}_{0}$ - $\mathrm{FePt}$ films, such as sputtering deposition [243 247] and molecular-beam epitaxy [248], finding that the asdeposited FePt films adopt the disordered structure A1, and in order to obtain the $\mathrm{L} 1_{0}$ phase, the film needs to be grown at high temperature $\left(500-700{ }^{\circ} \mathrm{C}\right)$ [243] or post-annealed at temperatures greater than $500{ }^{\circ} \mathrm{C}$.

However, if $\mathrm{L} 1_{0}-\mathrm{FePt}$ is desired to be integrated in MRAM devices, it is quite important to control its growth at low temperature, thus the structural ordering temperature must be effectively reduced. This can be achieved by means of different procedures [249]:

- Introducing third elements such as $\mathrm{Au}[250,251], \mathrm{Cu}[252]$ or $\mathrm{Zr}$ [247].

- "Tuning" the epitaxial strain from the lattice mismatch, as it can favors the ordering of the FePt films with c axis perpendicular to the film surface plane [253]. This can be performed by using different substrates such as $\mathrm{MgO}$ [249], and buffer materials, such as $\mathrm{Ag}, \mathrm{CrRu}$, oxidized $\mathrm{Si}$ [246], $\mathrm{Pt}$ [248, 249], Cr [254] or Au [249] among others.

- Annealing atomic-scale $\mathrm{Fe} / \mathrm{Pt}$ multilayers, as in the $\mathrm{L} 1_{0}$ phase the atomic arrangement of iron and platinum along the (001) direction consists on atomic-scale $\mathrm{Fe} / \mathrm{Pt}$ multilayers. These can be deposited by sequential sputtering [235 237].

- Ion irradiation [255].

- High Ar gas pressure during deposition [254].

- In situ annealing [235, 236, 245].

In this chapter,the effect of different substrates and buffer layers in the growth of $\mathrm{L} 1_{0}$-ordered FePt films with thicknesses around $10 \mathrm{~nm}$ is studied, deposited by alternating RF-sputtering at the IMEM. By using this deposition method, the temperature required for the $\mathrm{L} 1_{0}$-ordering of the $\mathrm{FePt}$ is in the range of $400-450{ }^{\circ} \mathrm{C}$, lower than in the case of co-deposited FePt films [256]. 


\section{$4.2 \quad$ FePt film growth}

Our objective is the integration of $\mathrm{L}_{0}$-FePt in heterostructures with $\mathrm{Fe}_{3} \mathrm{O}_{4}$ electrode and $\mathrm{MgO}$ tunnel barrier for the future obtention of MTJs (see chapter 5. section 5.3).

Should the FePt film be used as a bottom electrode, due to the the optical lithography processing required for the MTJs fabrication, at least, a thickness of $30 \mathrm{~nm}$ and a smooth surface are required. However, a grain microstructure, due to high temperature growths, is usually associated to the high perpendicular anisotropy (high order parameter). Therefore, there is a compromise between samples with significant out-of-plane magnetic anisotropy and enough surface smoothness to allow the growth of high quality tunnel barrier and upper electrode.

If we choose the magnetite as the bottom electrode, being also no less than $30 \mathrm{~nm}$ thick, the FePt layer must have at least $15 \mathrm{~nm}$ of thickness, otherwise its magnetic signal would be overcome by the $\mathrm{Fe}_{3} \mathrm{O}_{4}$ signal. In this case, the FePt roughness is not as important as in the previous configuration, although a capping layer would be advisable in order to avoid contamination or damage of the FePt.

However, the standard $\mathrm{L} 1_{0}-\mathrm{FePt}$ films grown at the IMEM in Parma (Italy) had thicknesses of $10 \mathrm{~nm}$, being too thin for our purpose. Therefore, our efforts were directed towards the obtention of high quality $\mathrm{L}_{0}$-ordered $\mathrm{FePt}$ films with thicknesses higher than $10 \mathrm{~nm}$, starting from the deposition conditions already optimized at the IMEM-CNR [234, 235]. The samples were prepared on $\mathrm{MgO}(001)$ substrates varying growth parameters such as the substrate temperature and the annealing treatment. The deposition over buffer layers, and on other substrates such as STO and $\left(\mathrm{La}_{0.3} \mathrm{Sr}_{0.7}\right)\left(\mathrm{Al}_{0.5} \mathrm{Ta}_{0.35}\right) \mathrm{O}_{3}$ (LSAT) has been also performed.

All the FePt films were prepared by sequential RF sputtering of atomicscale multilayers, where the deposition of very thin Fe and Pt layers with nominal thickness of $\sim 2 \AA$ were alternated and repeated to obtain films with the desired thickness. The chosen ratio between Fe and Pt thickness corresponds to the nominal atomic composition $\mathrm{Fe}_{57} \mathrm{Pt}_{43}$, which was calculated accounting for the densities and atomic weights of the two metals. The base pressure was approximately $2.3 \times 10^{-8}$ Torr, and the deposition pressure of $1 \times 10^{-2}$ Torr was obtained by means of an Ar flux of $6.4 \mathrm{sccm}$. The targets were sputtered with different power in order to obtain similar growth rates for the two materials: 


\begin{tabular}{|c||c|c|}
\hline Material & Power $(\mathrm{W})$ & Dep. rate $(\mathrm{nm} / \mathrm{min})$ \\
\hline \hline $\mathrm{Fe}$ & 45 & 1.5 \\
\hline $\mathrm{Pt}$ & 34 & 2.2 \\
\hline
\end{tabular}

Table 4.1: Sputtering power and deposition rate for Fe and Pt.

\subsection{1 $\mathrm{MgO}(001) / / \mathrm{FePt}$}

In first place, standard $10 \mathrm{~nm}$-thick $\mathrm{FePt}$ films were grown on $\mathrm{MgO}(001)$ substrates, following the standard procedure optimized at the IMEM. Then, 15nm-thick FePt films were grown on $\mathrm{MgO}(001)$ substrates at different process temperatures and annealing treatments, and finally, some buffer layers were introduced in order to study the growth of $15 \mathrm{~nm}$-thick films onto them.

The in-plane $\mathrm{L} 1_{0}$ - FePt lattice parameter $\left(\mathrm{a}_{i n}=3.85 \AA\right)$ is smaller than the $\mathrm{MgO}(001)$ lattice parameter $(\mathrm{a}=4.21 \AA)$ with a mismatch of about $9.4 \%$. This produces a tensile strain in the film plane and thus, a compressive strain in the out-of-plane direction, which favors the out-of-plane orientation of the c axis. Therefore, the expected epitaxy relation is:

$$
\mathrm{MgO}(001)[100] / / \mathrm{L1}_{0}-\mathrm{FePt}(001)[100]
$$

\section{Standard $\mathrm{MgO}(001) / /(10 \mathrm{~nm})$ FePt growth}

A standard $\mathrm{MgO}(001) / /(10 \mathrm{~nm}) \mathrm{FePt}$ film was deposited at a substrate temperature of $450{ }^{\circ} \mathrm{C}$ and annealed in Ar, at the same sputtering pressure $(\mathrm{P}=$ $1 \times 10^{-2}$ Torr) for 85 minutes at the deposition temperature to improve the chemical order degree.

The sample was magnetically characterized by AGFM, measuring the magnetization loops in directions perpendicular and parallel to the film plane at room temperature, up to a maximum magnetic field of $2 \mathrm{~T}$ (Figure 4.2). The substrate contribution was measured separately in both directions and subtracted from the data prior to the analysis.

The hysteresis loops show that the easy magnetization direction is perpendicular to the film plane, with a saturation magnetization $\mathrm{M}_{S \perp}$ of $959 \mathrm{emu}$ $/ \mathrm{cm}^{3}$ and a coercive field $\mathrm{H}_{C \perp}$ of $5750 \mathrm{Oe}$, both measured in the perpendicular direction. The in-plane hysteresis loop shows a small ferromagnetic contribution with a remanence $\mathrm{M}_{R \|}$ of $42 \mathrm{emu} / \mathrm{cm}^{3}$, indicating a slight deviation from the $\mathrm{L} 1_{0}$ - ordering. The anisotropy field $\mathrm{H}_{A}$ is found to be $68 \mathrm{kOe}$, obtained by extrapolating the magnetization slope in the in-plane measurement to the $\mathrm{M}_{S \perp}$ value. 


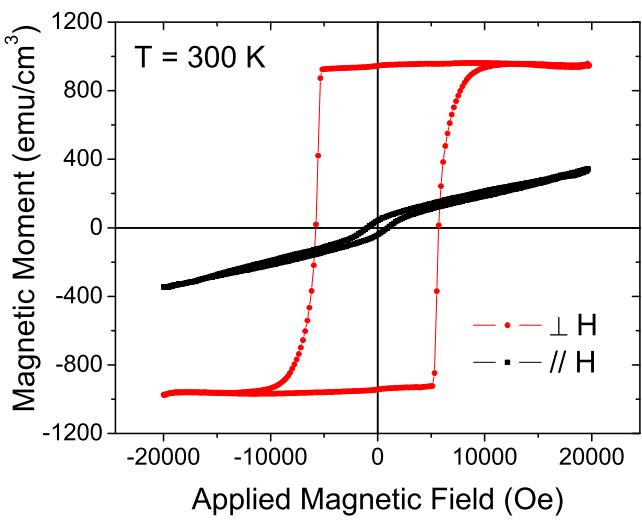

Figure 4.2: Magnetization of as a function of the magnetic field, applied both perpendicular and parallel to the sample surface.

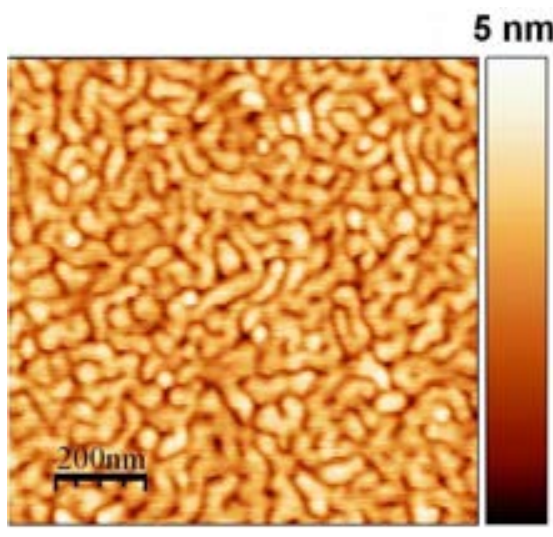

Figure 4.3: $1 \mu m \times 1 \mu m$ AFM image of a $\mathrm{MgO}(001) / /(10 \mathrm{~nm}) \mathrm{FePt}$ film in tapping mode with a $300 \mathrm{kHz}$ tip.

The surface characterization was performed by means of the Veeco AFM available at the IMEM research institute in Parma, in tapping mode with a $300 \mathrm{KHz}$ silicon tip (Figure 4.3), obtaining an Average Grain Size (AGS) of 60 $\mathrm{nm}$ and a RMS roughness of $0.82 \mathrm{~nm}$.

The magnetization and morphological results are in good agreement with those obtained for standard $\mathrm{MgO}(001) / /(10 \mathrm{~nm})$ FePt previously prepared at the IMEM.

\section{$\operatorname{MgO}(001) / /(15 n m)$ FePt growth}

(15 nm) FePt films were deposited on $\mathrm{MgO}(001)$ substrates at two different deposition temperatures $\mathrm{T}_{S}=400{ }^{\circ} \mathrm{C}$ and $450^{\circ} \mathrm{C}$, with and without annealing of 115 minutes in $\mathrm{Ar}$ at the sputtering process pressure $\left(\mathrm{P}=1 \times 10^{-2}\right.$ Torr $)$. A comparative study was performed in order to determine the growth conditions that produced the $(15 \mathrm{~nm})$ FePt film with the smoothest surface and the highest perpendicular orientation of the easy magnetization axis.

With this purpose, magnetization loops in directions perpendicular and parallel to the film plane at room temperature up to a maximum magnetic field of $2 \mathrm{~T}$ of all the samples were measured by means of AGFM, and AFM images were obtained in tapping mode. The main morphological and magnetic parameters for all the (15 nm) FePt films grown on $\mathrm{MgO}$ (001) are summarized in table 4.2 . 
As can be seen in Figure 4.4, the sample grown at $450{ }^{\circ} \mathrm{C}$ without annealing has the highest out-of-plane coercive field and the weakest in-plane signal, which indicates that the easy magnetization axis direction is perpendicular to the film surface. Indeed, provided that the saturation magnetization of all the samples are similar, the magnetocrystalline anisotropy of this film is one of the highest, as can be seen from the high anisotropy field.
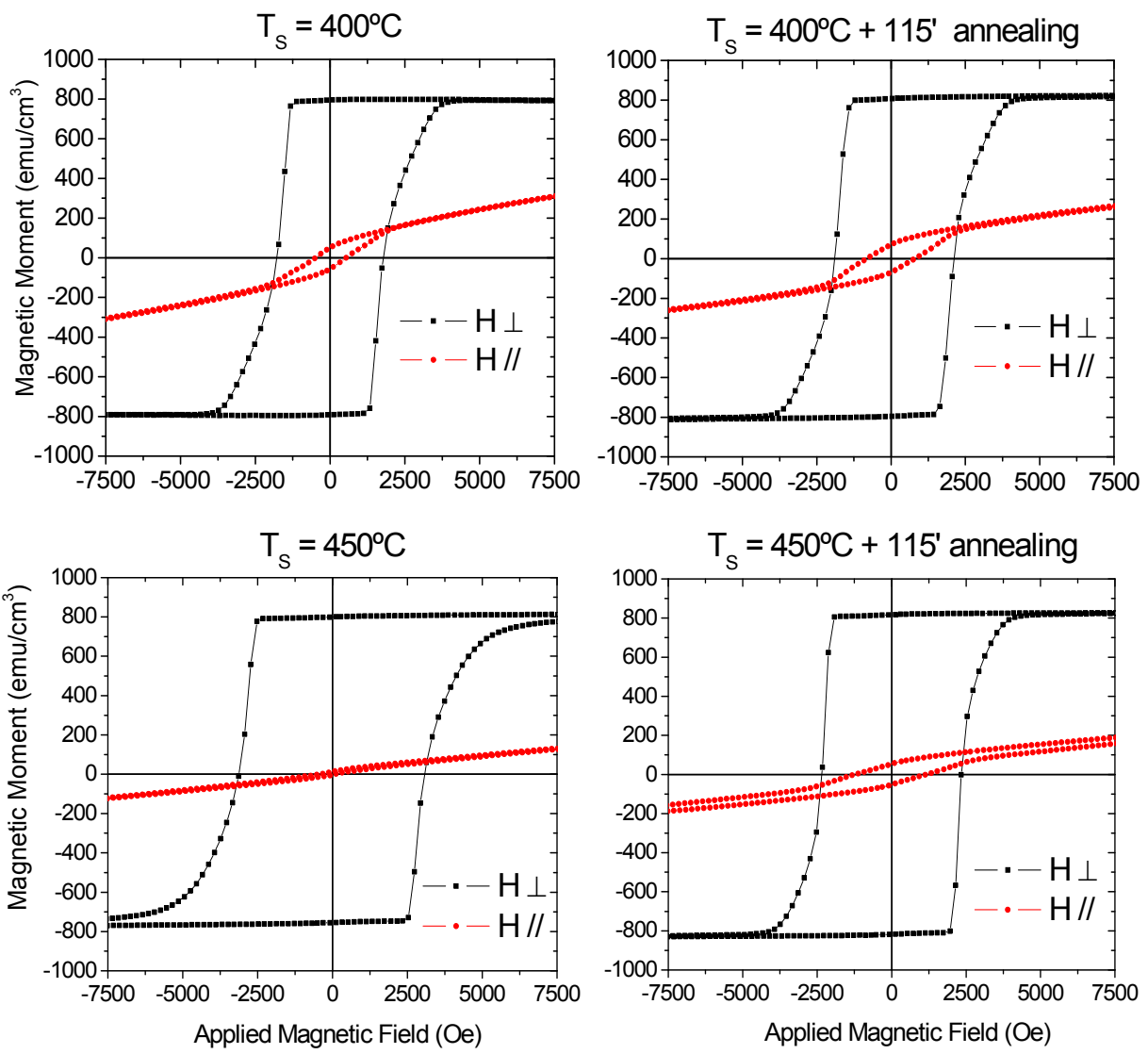

Figure 4.4: Magnetization measurements as a function of the applied magnetic field at room temperature of $\mathrm{MgO}(001) / /(15 \mathrm{~nm}) \mathrm{FePt}$ films with different thermal treatments. The magnetic field has been applied in the plane of the film surface (red) and perpendicularly (black).

Figure 4.5 shows the AFM images of the $(15 \mathrm{~nm})$ FePt films obtained in tapping mode over a surface of $1 \mu \mathrm{m} \times 1 \mu \mathrm{m}$. It can be observed that, as was pointed out before, a grain microstructure of the FePt films is associated to the out-of-plane magnetic anisotropy. Since all the RMS roughnesses are of the same order, ranging from 0.3 to $0.6 \mathrm{~nm}$, and taking into account the magnetic measurements, the sample grown at $450{ }^{\circ} \mathrm{C}$ without annealing seems to be the film with the highest quality. 

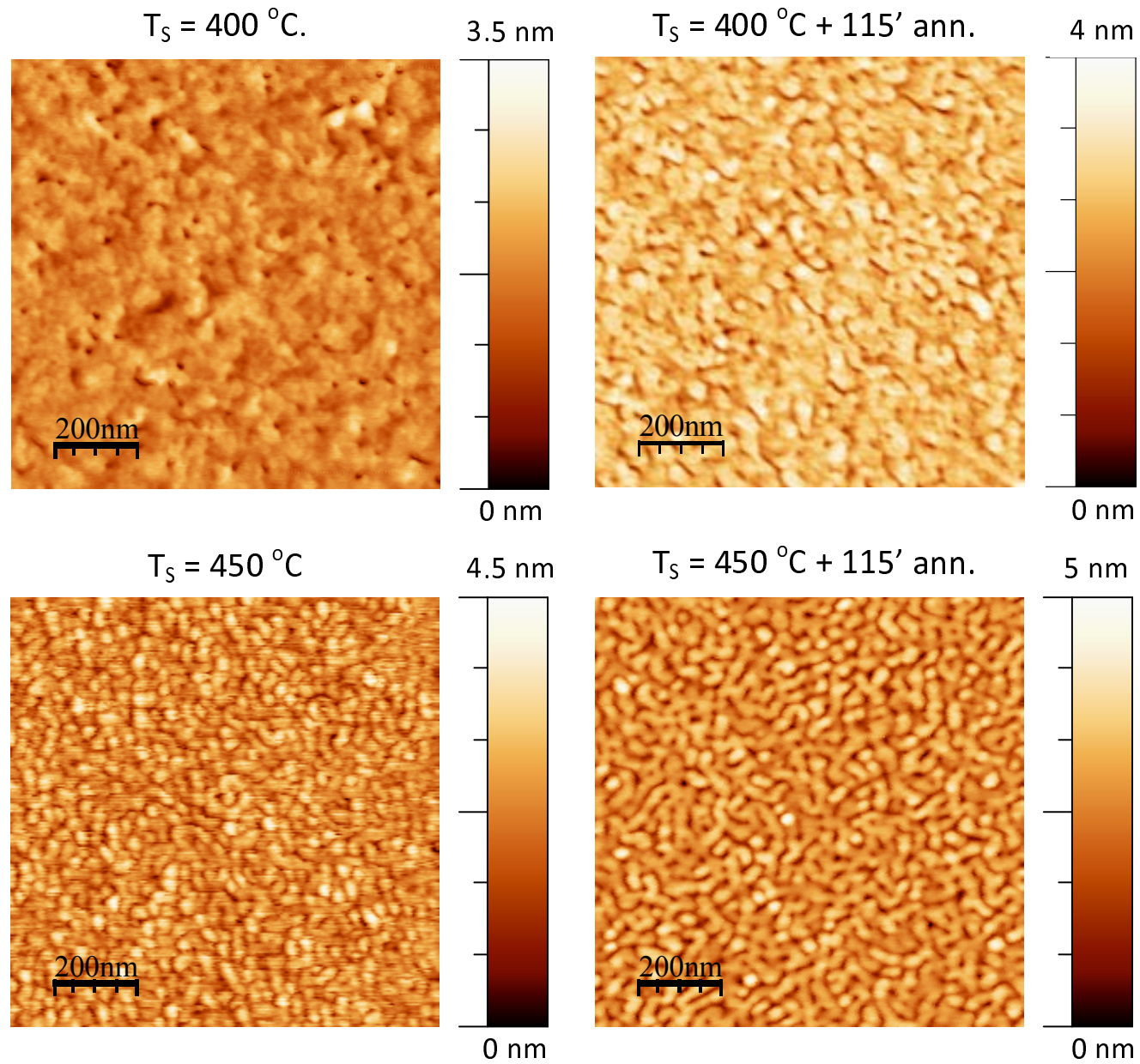

Figure 4.5: AFM images at room temperature in tapping mode of $\mathrm{MgO}(001) / /(15$ nm) FePt films with different thermal treatments.

\begin{tabular}{|c||c|c|c|c||c|c|}
\hline $\begin{array}{c}\text { Thermal } \\
\text { treatment }\end{array}$ & $\begin{array}{c}\mathrm{M}_{S \perp} \\
\left(\mathrm{emu} / \mathrm{cm}^{3}\right)\end{array}$ & $\begin{array}{c}\mathrm{M}_{R \|} \\
\left(\mathrm{emu} / \mathrm{cm}^{3}\right)\end{array}$ & $\begin{array}{c}\mathrm{H}_{C \perp} \\
(\mathrm{kOe})\end{array}$ & $\begin{array}{c}\mathrm{H}_{A} \\
(\mathrm{kOe})\end{array}$ & $\begin{array}{c}\text { RMS } \\
(\mathrm{nm})\end{array}$ & $\begin{array}{c}\text { AGS } \\
(\mathrm{nm})\end{array}$ \\
\hline \hline $\mathrm{T}_{S}=400^{\circ} \mathrm{C}$ & 792 & 53 & 1.8 & 39.4 & 0.3 & - \\
\hline $\mathrm{T}_{S}+115^{\prime}$ ann. & 817 & 70 & 2.0 & 66.3 & 0.4 & 60 \\
\hline \hline $\mathrm{T}_{S}=450^{\circ} \mathrm{C}$ & 790 & 8 & 3.1 & 61.4 & 0.5 & 40 \\
\hline $\mathrm{T}_{S}+115^{\prime}$ ann. & 832 & 30 & 2.3 & 45.7 & 0.6 & 30 \\
\hline
\end{tabular}

Table 4.2: Magnetic and morphological parameters obtained from the hysteresis loops and AFM images, respectively. 
The characterization of the (15 nm) FePt sample grown on the $\mathrm{MgO}$ (001) substrate at the temperature of $450{ }^{\circ} \mathrm{C}$ was completed by performing XRD experiments at the Bruker diffractometer available at the INA in Zaragoza (Spain). In the XRD measurement, only the diffraction peaks corresponding to the $\mathrm{L} 1_{0}-\mathrm{FePt}(001)$ were observed (see figure 4.6), indicating the (001) orientation of the FePt film, its $\mathrm{L} 1_{0}$ ordering and the absence of other crystalline phases.

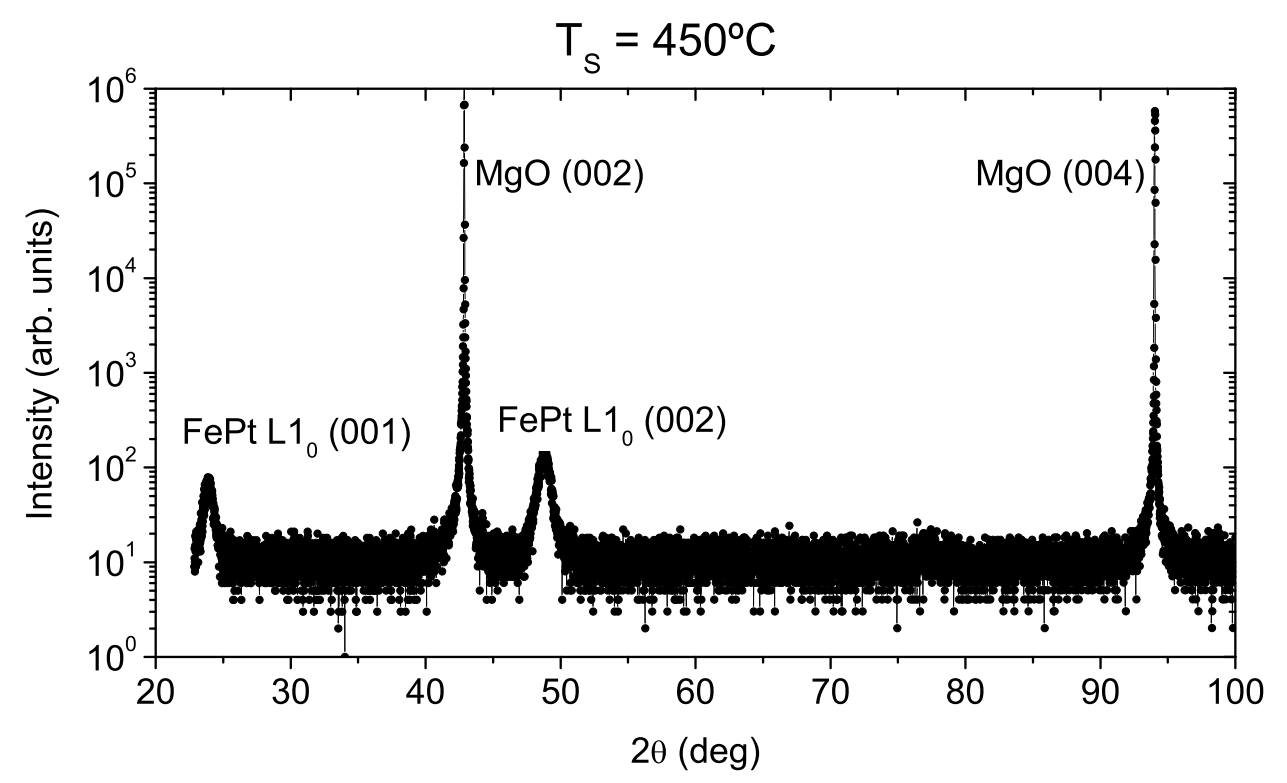

Figure 4.6: XRD pattern of the $\mathrm{MgO}(001) / /(15 \mathrm{~nm}) \mathrm{FePt}$ film grown at a substrate temperature of $450{ }^{\circ} \mathrm{C}$ without annealing.

As a conclusion, we have optimized the deposition conditions for $15 \mathrm{~nm}$ thick $\mathrm{L}_{0}$ - FePt films on $\mathrm{MgO}(001)$ substrates, finding the highest quality in the sample grown at the substrate temperature of $450{ }^{\circ} \mathrm{C}$ without annealing.

This film is suitable for its inclusion in MTJ with magnetite bottom electrode. However, if the FePt film is wanted to be used as the bottom electrode and due to lithography process requirements, it is necessary to have a conductive bottom layer of at least $30 \mathrm{~nm}$. This could be overcome by growing $15 \mathrm{~nm}$-thick $\mathrm{L}_{0}$ - FePt films over a metallic buffer (Pt or $\mathrm{Au}$ ), study that is detailed in the following section. 


\subsection{2 $\mathrm{MgO}(001) / /$ Buffer layer / FePt}

\section{Pt and Au buffer deposition}

Previously to the deposition of the $\mathrm{MgO}$ (001) // Buffer layer / FePt heterostructure, an independent study of the different buffer materials is advisable. Therefore, $\mathrm{Pt}$ and $\mathrm{Au}$ films were deposited on $\mathrm{MgO}$ (001) substrates and studied by XRD and AFM in order to study their structural and surface characteristics. As the FePt optimized substrate temperature for a thickness of $15 \mathrm{~nm}$ is $450{ }^{\circ} \mathrm{C}$, it is reasonable to deposit the buffer layers at the same temperature, and considering both the lithography process requirements and the necessity of a significant signal for the XRD pattern, we considered that a thickness of $100 \mathrm{~nm}$ was acceptable.

Pt and Au 100 nm-thick-films were chosen as buffer materials because they are expected to be suitable candidates, as both of them are cubic and their lattice parameters values are between the $\mathrm{MgO}$ and the FePt values:

$$
\begin{array}{rlrl}
\mathrm{a}_{M g O} & =4.21 \AA & & \mathrm{a}_{F e P t}=3.85 \AA \\
\mathrm{a}_{P t} & =3.92 \AA & \mathrm{a}_{A u}=4.08 \AA
\end{array}
$$

The buffer materials were deposited by means of RF-sputtering following the same procedure optimized for the FePt growth, adjusting the discharge power to obtain the same deposition rate for both films $(2 \mathrm{~nm} / \mathrm{min})$ :

$$
\mathrm{P}_{P t}=37 \mathrm{~W} \quad \mathrm{P}_{A u}=22 \mathrm{~W}
$$

XRD patterns of both films were measured in an standard x-ray powder diffractometer at the Chemistry department in the University of Parma. The disorder degree of our samples can be estimated by comparison between the relative intensity of the diffraction peaks in our patterns and in a typical polycrystalline diffraction pattern (see Table 4.3).

Therefore, if the Pt film was completely polycrystalline, the relative intensity between the (111) and the (200) would be:

$$
I_{R}^{\text {poly }}\left(\frac{111}{200}\right)=\frac{1000}{476}=2.1
$$

Were the sample completelly (200) oriented (thus, (002)):

$$
I_{R}^{\text {oriented }}\left(\frac{111}{200}\right)=\frac{0}{I}=0
$$




\begin{tabular}{|c|c|c||c|c|c|}
\hline \multicolumn{3}{|c||}{ Pt } & \multicolumn{3}{c|}{ Au } \\
\hline $2 \theta($ deg $)$ & $I$ (a.u. $)$ & $($ hkl $)$ & $2 \theta($ deg $)$ & $I($ a.u. $)$ & $($ hkl $)$ \\
\hline 39.763 & 1000 & 111 & 38.187 & 1000 & 111 \\
46.244 & 476 & 200 & 44.385 & 479 & 200 \\
67.469 & 277 & 220 & 64.576 & 284 & 220 \\
81.265 & 308 & 311 & 77.566 & 313 & 311 \\
85.712 & 89 & 222 & 81.722 & 89 & 222 \\
103.511 & 47 & 400 & 98.127 & 44 & 400 \\
117.707 & 178 & 331 & 110.816 & 158 & 331 \\
& & & 115.259 & 157 & 420 \\
\hline
\end{tabular}

Table 4.3: XRD pattern of polycrystalline $\mathrm{Pt}$ and $\mathrm{Au}$.

From the diffraction pattern (Figure 4.7) is observed that the Pt film is (001)-oriented, with a small (111) contribution that can be calculated from the experimental relative intensity of the diffraction peaks as:

$$
\%(111)=\frac{I_{R}^{\exp }\left(\frac{111}{200}\right)}{I_{R}^{\text {poly }}\left(\frac{111}{200}\right)} \cdot 100 \%=\frac{0.022}{2.1} \cdot 100 \% \simeq 1 \%
$$

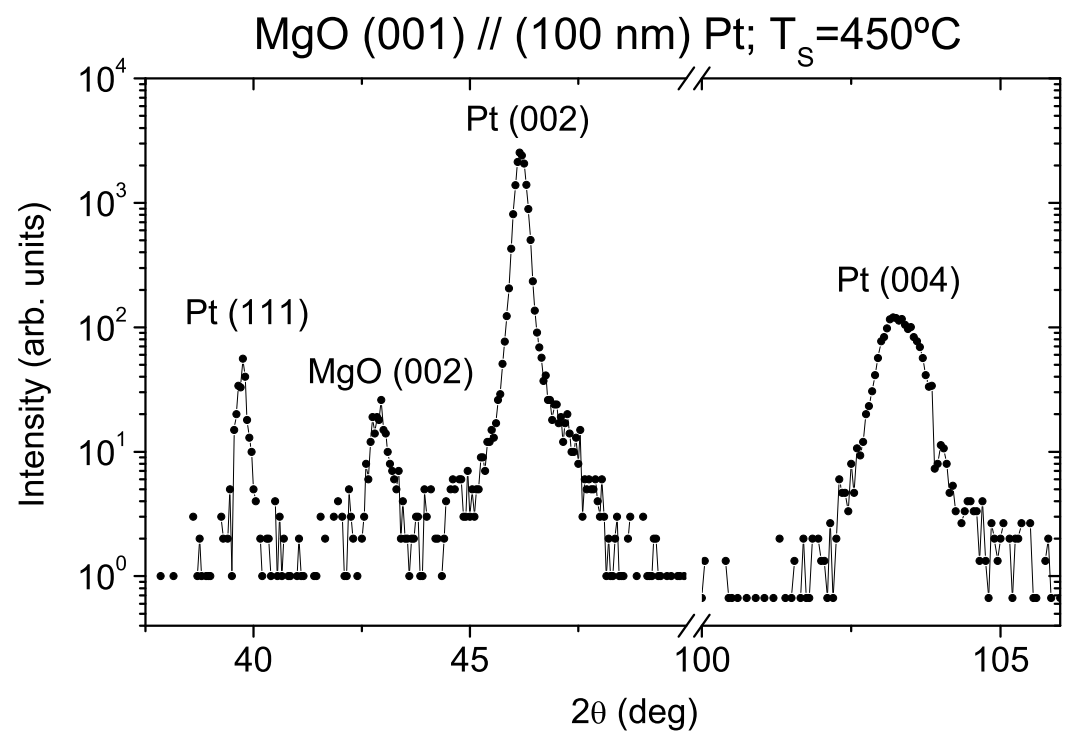

Figure 4.7: XRD pattern of the $\mathrm{MgO}(001) / /(100 \mathrm{~nm})$ Pt film, showing a (001) orientation with a small disorder degree in the (111) direction. 
The same calculation can be performed for the Au film. From its diffraction pattern shown in figure 4.8, it can be observed that the film is (111)-oriented with a small (001) contribution, thus, the disorder degree can be calculated as:

$$
\begin{gathered}
I_{R}^{\text {poly }}\left(\frac{200}{111}\right)=\frac{1000}{479}=0.5 \\
\%(001)=\frac{I_{R}^{\exp }\left(\frac{002}{111}\right)}{I_{R}^{\text {poly }}\left(\frac{200}{111}\right)} \cdot 100 \%=\frac{0.04}{0.5} \cdot 100 \%=8 \%
\end{gathered}
$$

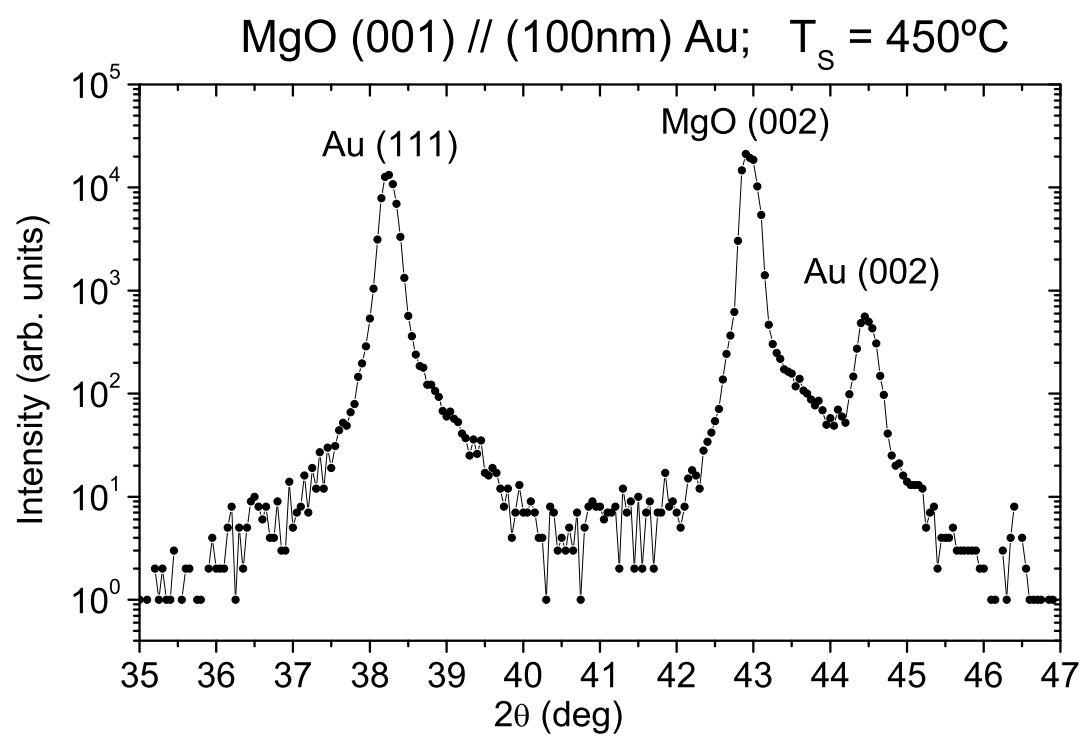

Figure 4.8: XRD pattern of the $\mathrm{MgO}(001) / /(100 \mathrm{~nm}) \mathrm{Au})$ film, showing a (111) orientation with a small disorder degree in the (001) direction.

The buffer growth orientations on the $\mathrm{MgO}$ (001) substrates are therefore, $\mathrm{MgO}(001) / /(100 \mathrm{~nm}) \mathrm{Pt}(001)$ and $\mathrm{MgO}(001) / /(100 \mathrm{~nm}) \mathrm{Au}$ (111), with an acceptable crystalline quality, as it can be observed in the XRD measurements. The next issue that must be accounted for in order to grow the FePt film over the buffer surface is its surface topography. With this purpose, AFM images were obtained in tapping mode from both films.

The AFM image of the $100 \mathrm{~nm}$-thick Pt film shows a surface topography with relatively low roughness $(\mathrm{RMS}=0.4 \mathrm{~nm}$ ) (Figure 4.9), while the image from the $100 \mathrm{~nm}$-thick $\mathrm{Au}$ film indicates a surface composed by grains with sizes between 0.5 and $1 \mu \mathrm{m}$ diameter and flat surfaces, which produces a high average roughness $(\mathrm{RMS}=28.2 \mathrm{~nm})$ (Figure 4.10). 

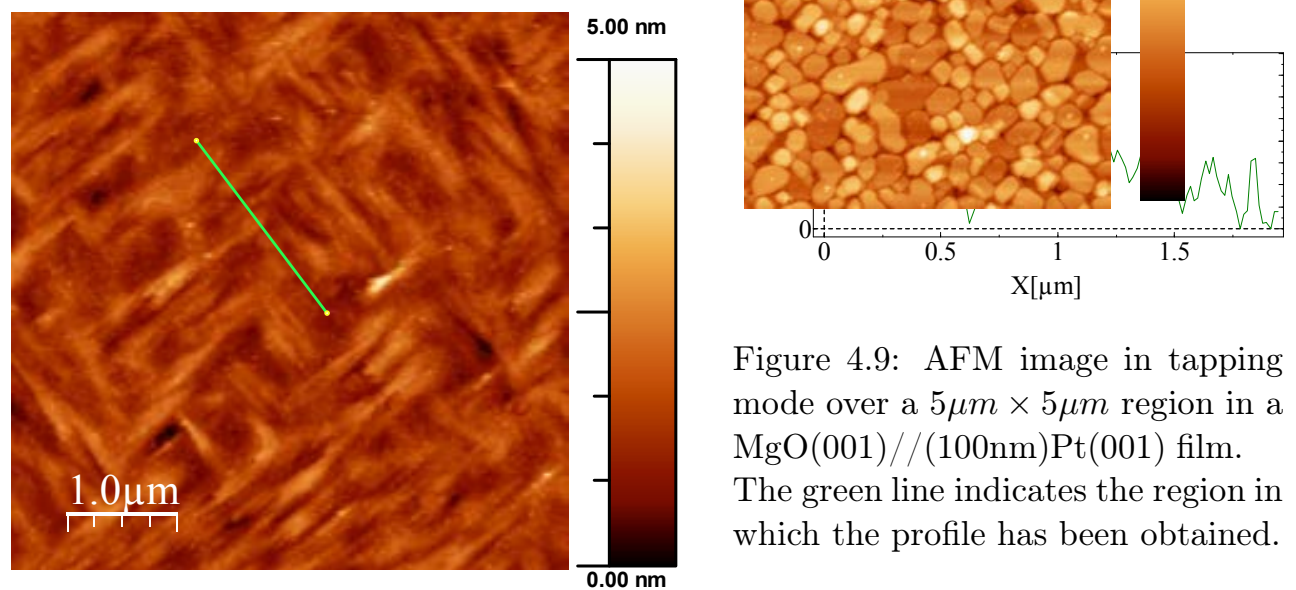

Figure 4.9: AFM image in tapping mode over a $5 \mu m \times 5 \mu m$ region in a $\mathrm{MgO}(001) / /(100 \mathrm{~nm}) \mathrm{Pt}(001)$ film.

The green line indicates the region in which the profile has been obtained.
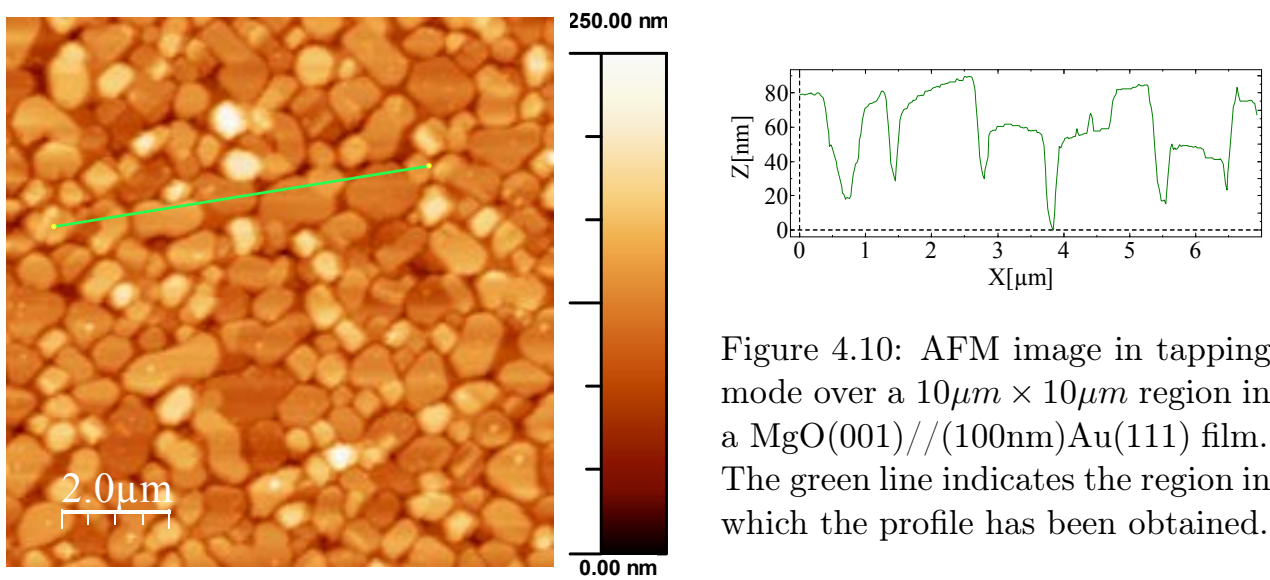

Figure 4.10: AFM image in tapping mode over a $10 \mu m \times 10 \mu m$ region in a $\mathrm{MgO}(001) / /(100 \mathrm{~nm}) \mathrm{Au}(111)$ film. The green line indicates the region in which the profile has been obtained.

Therefore, we consider that the $\mathrm{Pt}$ is more suitable than the $\mathrm{Au}$ as a buffer material, as a low roughness bottom electrode is necessary for the subsequent growth of high quality heterostructures. 


\section{$\mathrm{MgO}(001) / /(100 \mathrm{~nm}) \mathrm{Pt}(001) /(15 \mathrm{~nm}) \mathrm{FePt}$}

A FePt film with a thickness of $15 \mathrm{~nm}$ was deposited over a $\mathrm{Pt}$ buffer film with $100 \mathrm{~nm}$ thickness on an $\mathrm{MgO}$ (001) substrate; both layers were grown with RF-sputtering at a substrate temperature of $450{ }^{\circ} \mathrm{C}$, with the previously detailed sputtering parameters.

The magnetic characterization was performed by means of AGFM at room temperature, with the magnetic field applied parallel to the sample surface and afterwards perpendicular to it, up to a maximum magnetic field of $2 \mathrm{~T}$ (see Fig. 4.11). The saturation magnetization $\mathrm{M}_{S}$ was found at $600 \mathrm{emu} / \mathrm{cm}^{3}$, lower than the value obtained for $(15 \mathrm{~nm}) \mathrm{FePt}$ films grown without any buffer layer (around $800 \mathrm{emu} / \mathrm{cm}^{3}$ ), also finding a lower coercive field $\mathrm{H}_{C}=756$ Oe.

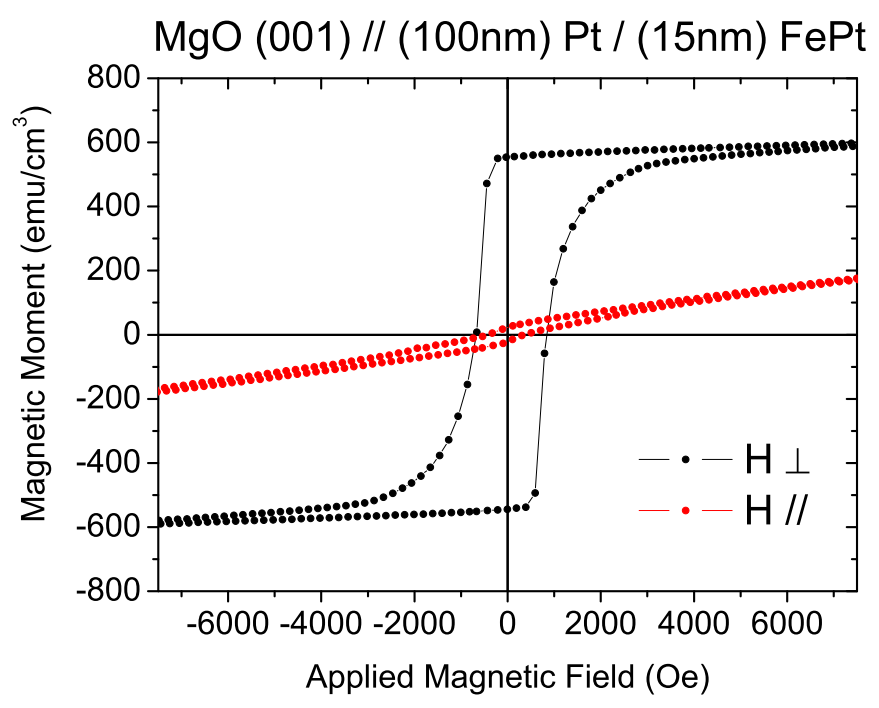

Figure 4.11: Magnetization measurements as a function of the applied magnetic field at room temperature of a $\mathrm{MgO}(001) / /(100 \mathrm{~nm}) \mathrm{Pt} /(15 \mathrm{~nm}) \mathrm{FePt}$ film. The magnetic field has been applied in the plane of the film surface (red) and perpendicularly (black).

The AFM images obtained in tapping mode indicated the high roughness of the film $(\mathrm{RMS}=2.9 \mathrm{~nm})$, which presented material aggregations in its surface, thus demonstrating that the deposition of $15 \mathrm{~nm}$ of FePt on a buffer Pt layer under our growth conditions provided a sample that is not suitable for its integration in heterostructures (see Fig. 4.12).

Therefore, other substrate temperatures or annealings should be studied in order to optimize the growth of the $\mathrm{MgO}$ (001) // Buffer / FePt system. 


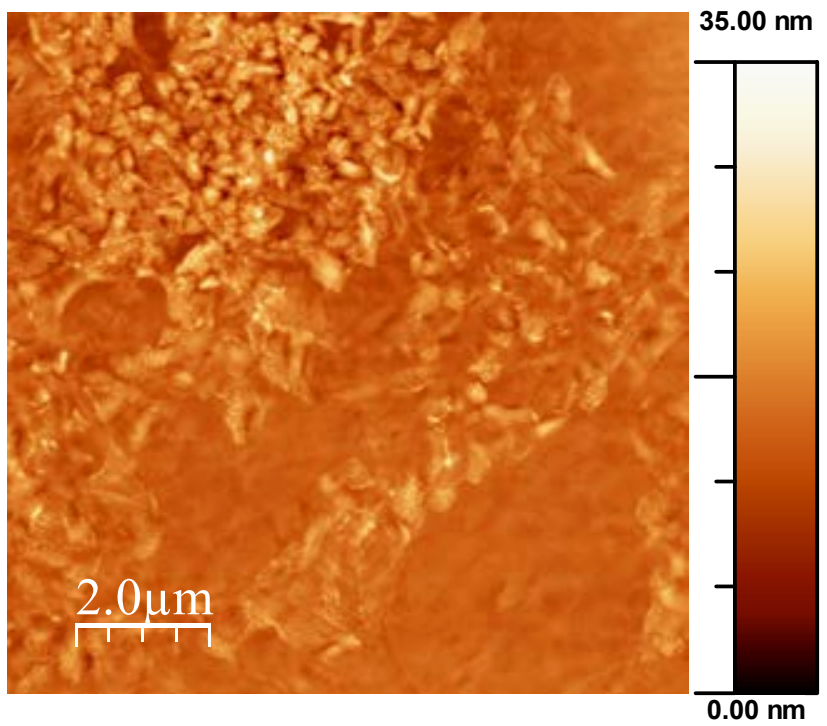

Figure 4.12: AFM images at room temperature in tapping mode of a $\mathrm{MgO}(001) / /$ (100 nm) Pt / (15 nm) FePt film.

\subsection{3 $\left(\mathrm{La}_{0.3} \mathrm{Sr}_{0.7}\right)\left(\mathrm{Al}_{0.5} \mathrm{Ta}_{0.35}\right) \mathrm{O}_{3}(110) / /$ FePt}

LSAT (110) substrates have a lattice parameter $a_{L S A T}$ of $3.87 \AA$, producing a lattice mismatch with the FePt of a $0.5 \%$, much smaller than in the case of growth on $\mathrm{MgO}(001)$ substrates. As the in-plane $\mathrm{L} 1_{0}-$ FePt lattice parameter $\left(\mathrm{a}_{\text {in }}=3.85 \AA\right)$ is smaller than $\mathrm{a}_{L S A T}$ lattice parameter, it produces a tensile strain in the film plane and a compressive strain in the out of plane direction which favors the out-of-plane orientation of the $\mathrm{c}$ axis. Therefore, this substrate is expected to be suitable for the growth of $\mathrm{L}_{0}$ - ordered FePt films.

A $15 \mathrm{~nm}$-thick film of FePt was deposited on an LSAT (110) substrate with the growth conditions optimized for $\mathrm{MgO}(001) / /(15 \mathrm{~nm}) \mathrm{FePt}$ films: substrate temperature of $450{ }^{\circ} \mathrm{C}$ without annealing after the deposition (see section 4.2.1). Afterwards, the sample was magnetically and morphologically characterized by means of AGFM and AFM respectively.

The AGFM measurements were performed at room temperature, with the magnetic field applied both in the plane of the film surface and perpendicularly to it, up to a maximum applied field of $2 \mathrm{~T}$ (Figure 4.13). Although the saturation magnetization was as high as the previously optimized FePt films $\left(800 \mathrm{emu} / \mathrm{cm}^{3}\right)$, the easy direction of the magnetization is not perpendicular to the film surface, indeed, it is mostly tilted towards the film surface film. This fact, together with the low coercivity $\left(\mathrm{H}_{C}=700 \mathrm{Oe}\right)$, make the LSAT // $(15 \mathrm{~nm}) \mathrm{FePt}$ sample inadequate for our objective. 


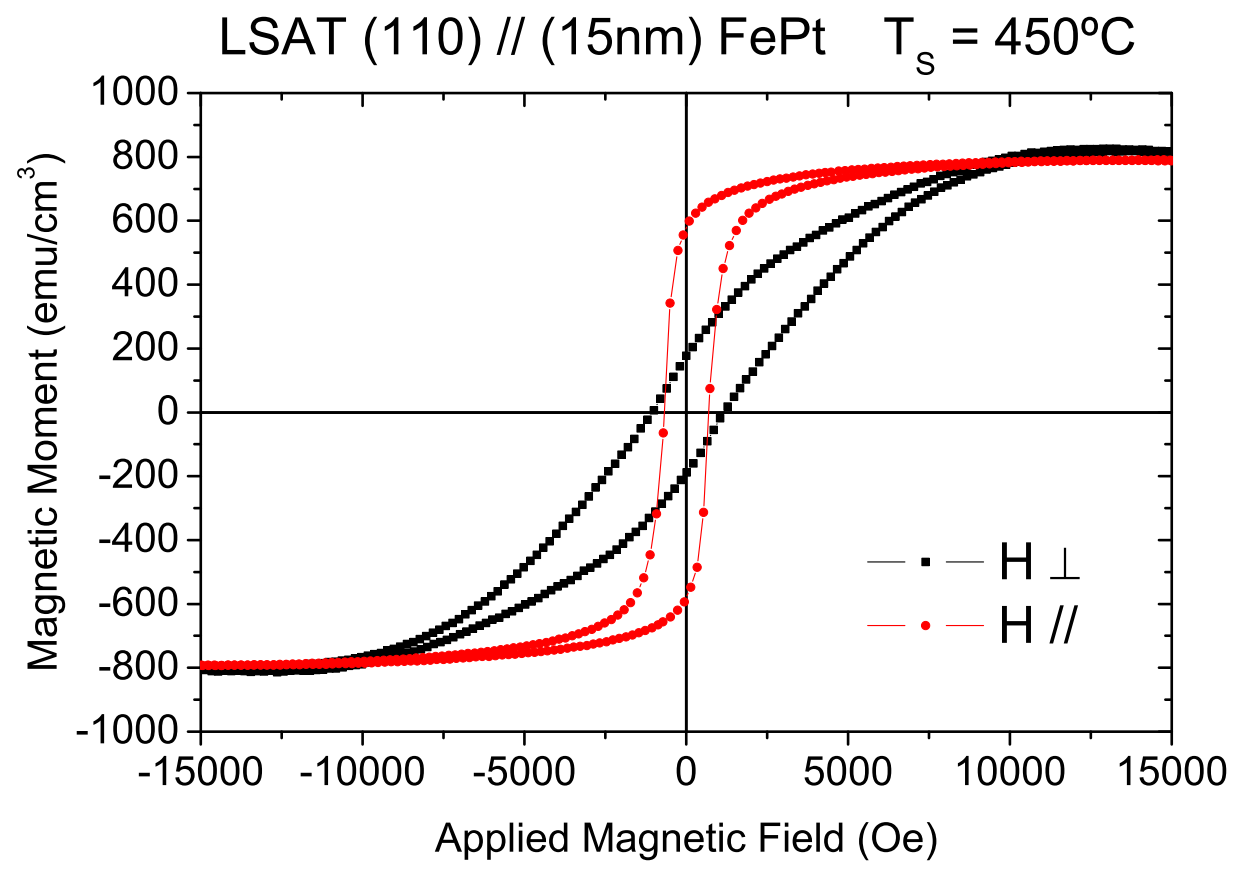

Figure 4.13: Magnetization measurements as a function of the applied magnetic field at room temperature of an LSAT (110) // (15 nm) FePt film. The magnetic field has been applied in the plane of the film surface (red) and perpendicularly (black).

The easy magnetization direction indicates that the FePt may have grown with a cubic structure on the LSAT substrate, although further structural characterization such as XRD should be performed in order to clarify this point. This could be due to the small mismatch between the LSAT and the $\mathrm{FePt}$, which favors the $\mathrm{A} 1$ growth of the FePt or the $\mathrm{L} 1_{0}$ structure with caxis not preferentially oriented to the film plane, with respect to the desired (001)-oriented $\mathrm{L} 1_{0}$ structure.

AFM images performed in tapping mode over a surface of $1 \mu \mathrm{m} \times 1 \mu \mathrm{m}$ indicate the a roughness $(\mathrm{RMS}=0.3 \mathrm{~nm})$ and uniform microstructure of the film (Figure 4.14). Although the morphology of the sample is suitable for its inclusion in a magnetic tunnel junction, its magnetic behavior does not meet the requirements for a perpendicular recording device. Therefore, further studies on other substrate temperatures or annealing treatments could lead to more adequate magnetic properties. 


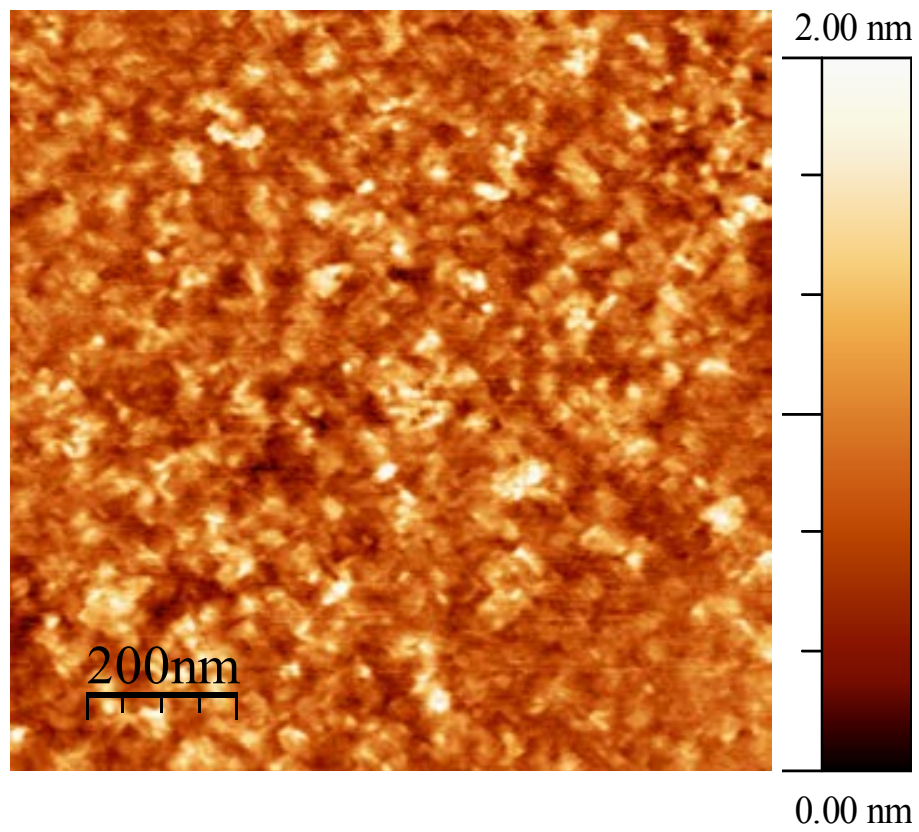

Figure 4.14: $1 \mu m \times 1 \mu m$ AFM image at room temperature in tapping mode of a LSAT $(110) / /(15 \mathrm{~nm}) \mathrm{FePt}$ film deposited at a substrate temperature of $450{ }^{\circ} \mathrm{C}$.

\subsubsection{STO (001) // FePt}

STO (001) substrates have a lattice parameter a $\mathrm{STO}_{\text {of }} 3.91 \AA$, producing a lattice mismatch of $1.5 \%$ with the FePt and a tensile strain in the film plane and compressive strain in the out of plane direction, favoring the outof-plane orientation of the $\mathrm{c}$ axis in the FePt. STO substrates are specially interesting for the growth of FePt films because, once the deposition conditions are optimized, they are also valid for the deposition on the conductor Nb:STO substrates. The problem of the thin bottom electrode in lithography would be then avoided, since the conductive bottom electrode would be the whole substrate.

15 nm-thick FePt films were grown on STO (001) substrates at two different temperatures: 350 and $450{ }^{\circ} \mathrm{C}$. The samples were magnetically characterized by means of AGFM at room temperature by applying the magnetic field both in the plane of the film surface and perpendicular to it, up to a maximum magnetic field of $2 \mathrm{~T}$ (Figure 4.15). Although the magnetization saturation of both samples is similar (around $850 \mathrm{emu} / \mathrm{cm}^{3}$ ), the coercive field is bigger for the film grown at $450{ }^{\circ} \mathrm{C}$, finding $\mathrm{H}_{C}=1740 \mathrm{Oe}$, while for the lower temperature the value is $\mathrm{H}_{C}=390$ Oe. Indeed, the magnetocrystalline anisotropy is also higher in the sample deposited at the highest substrate temperature. 

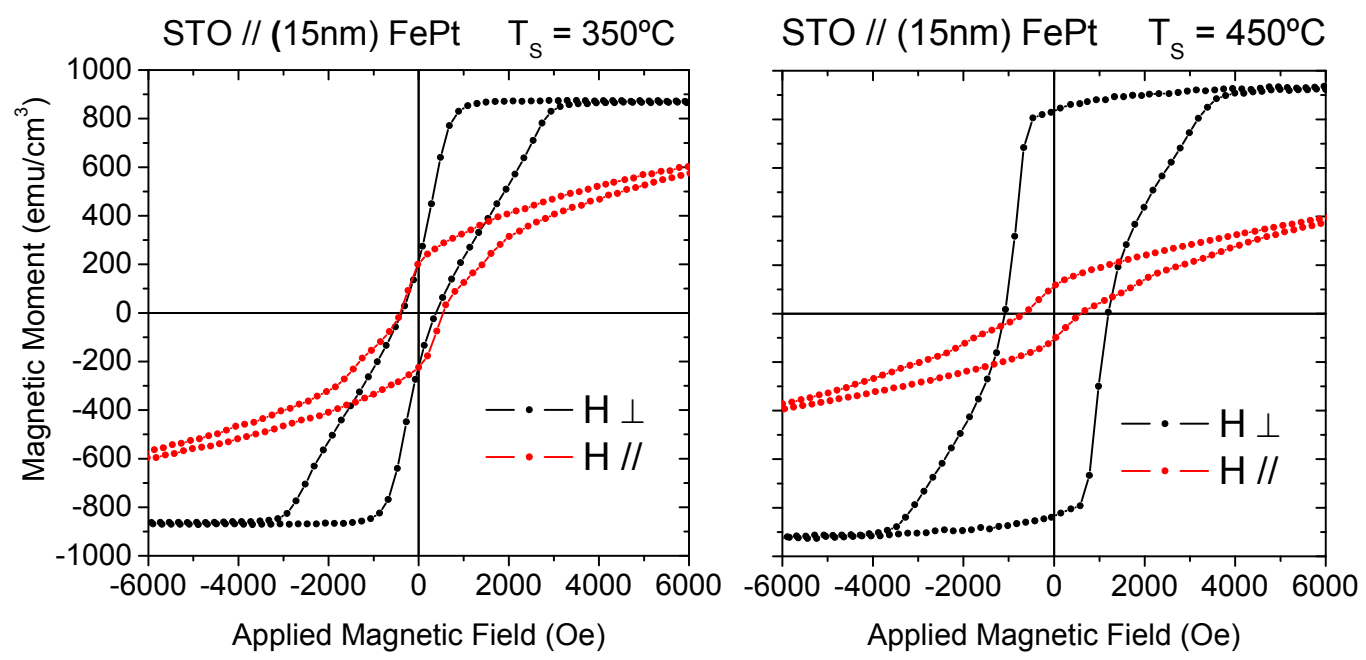

Figure 4.15: Magnetization measurements as a function of the applied magnetic field at room temperature of STO (001) // (15 nm) FePt films grown at different substrate temperatures. The magnetic field has been applied in the plane of the film surface (red) and perpendicularly (black).

AFM images from the STO $(001) / /(15 \mathrm{~nm}) \mathrm{FePt}$ film grown at $450{ }^{\circ} \mathrm{C}$ were obtained in tapping mode at room temperature (Figure 4.16), showing the homogeneity and low roughness of the surface $(\mathrm{RMS}=0.2 \mathrm{~nm})$. The MFM image of the film in the demagnetized state shows the perpendicular domains with irregularities both in the domain shape and size.
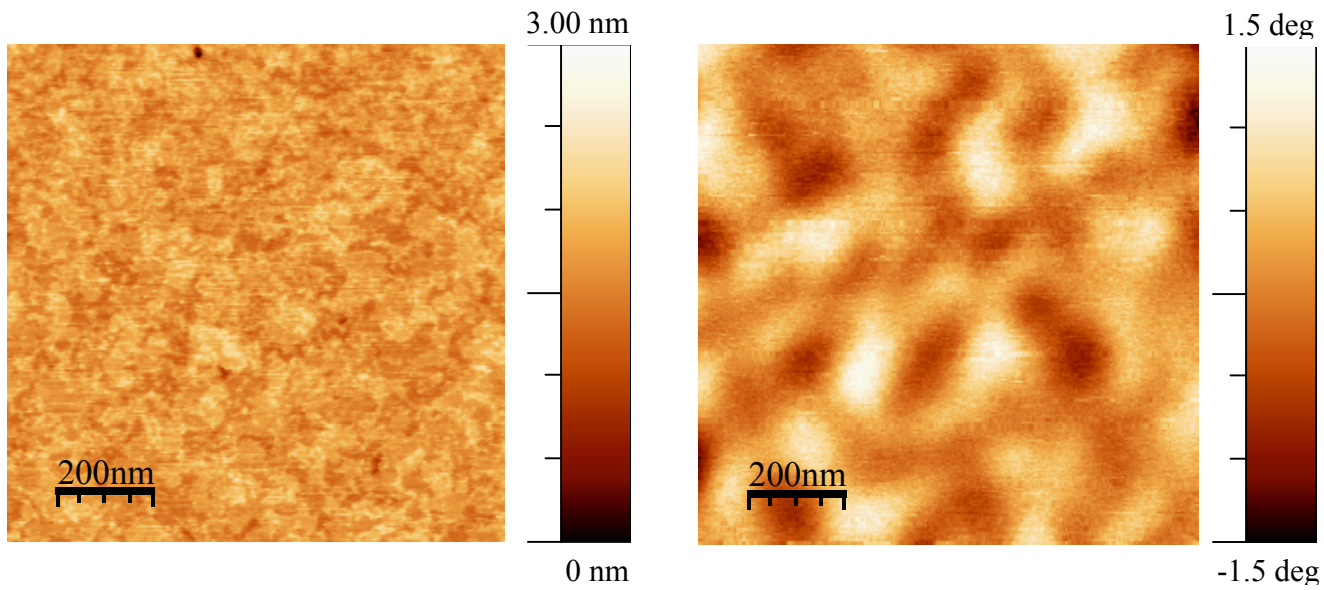

Figure 4.16: Left panel: tapping mode AFM image of the STO (001) // (15 nm) FePt film over a region of $1 \mu m \times \mu m$. Right panel: MFM image obtained simultaneously to the AFM measurement. 
The XRD measurement show all the (001) reflections of the $\mathrm{L} 1_{0}-\mathrm{FePt}$, indicating the epitaxial growth of the FePt film on the STO (001) and the absence of other crystallographic phases (Figure 4.17).

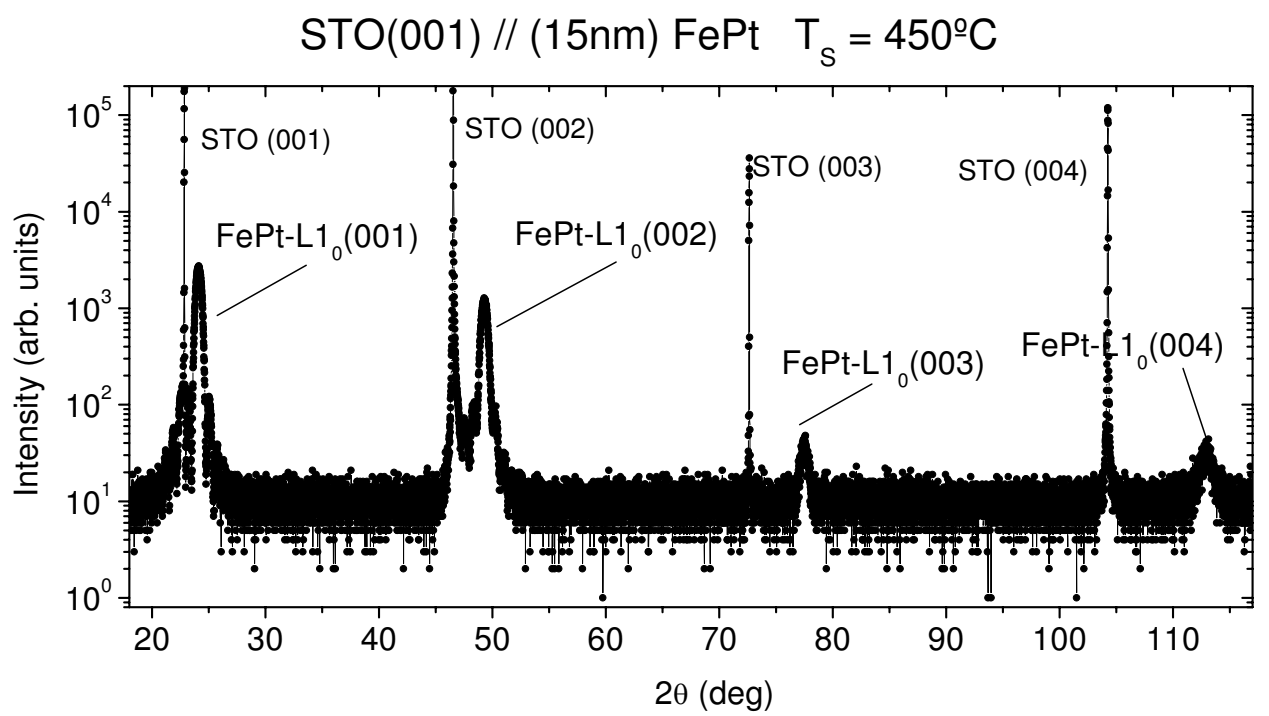

Figure 4.17: X-ray Diffraction pattern of the STO (001) // (15 nm) FePt film obtained at the Bruker XRD equipment at the INA (Spain).

A $15 \mathrm{~nm}$-thick FePt film was grown on STO (001) at a substrate temperature of $450{ }^{\circ} \mathrm{C}$ and annealed in $\mathrm{Ar}$ at the deposition pressure and substrate temperature for 60 minutes in order to improve both the anisotropy and the crystalline quality of the film.

The magnetic measurements performed by means of AGFM (Figure 4.18) show an increase in the magnetization saturation $\left(\mathrm{M}_{S}=910 \mathrm{emu} / \mathrm{cm}^{3}\right)$ and the coercive field $\left(\mathrm{H}_{C}=2720 \mathrm{Oe}\right)$ compared with the values obtained for the sample deposited at the same temperature without annealing. The perpendicular anisotropy is also improved, as although a small parallel remanence is observed, the anisotropy field increases up to $91.7 \mathrm{kOe}$.

The AFM images obtained in tapping mode confirm the presence of the granular morphology associated to the existence of perpendicular anisotropy, with an RMS roughness of $0.7 \mathrm{~nm}$ and an AGS of 70nm (Figure 4.19). The MFM image of the film in the demagnetized state shows the irregularly shaped and sized perpendicular domains. 


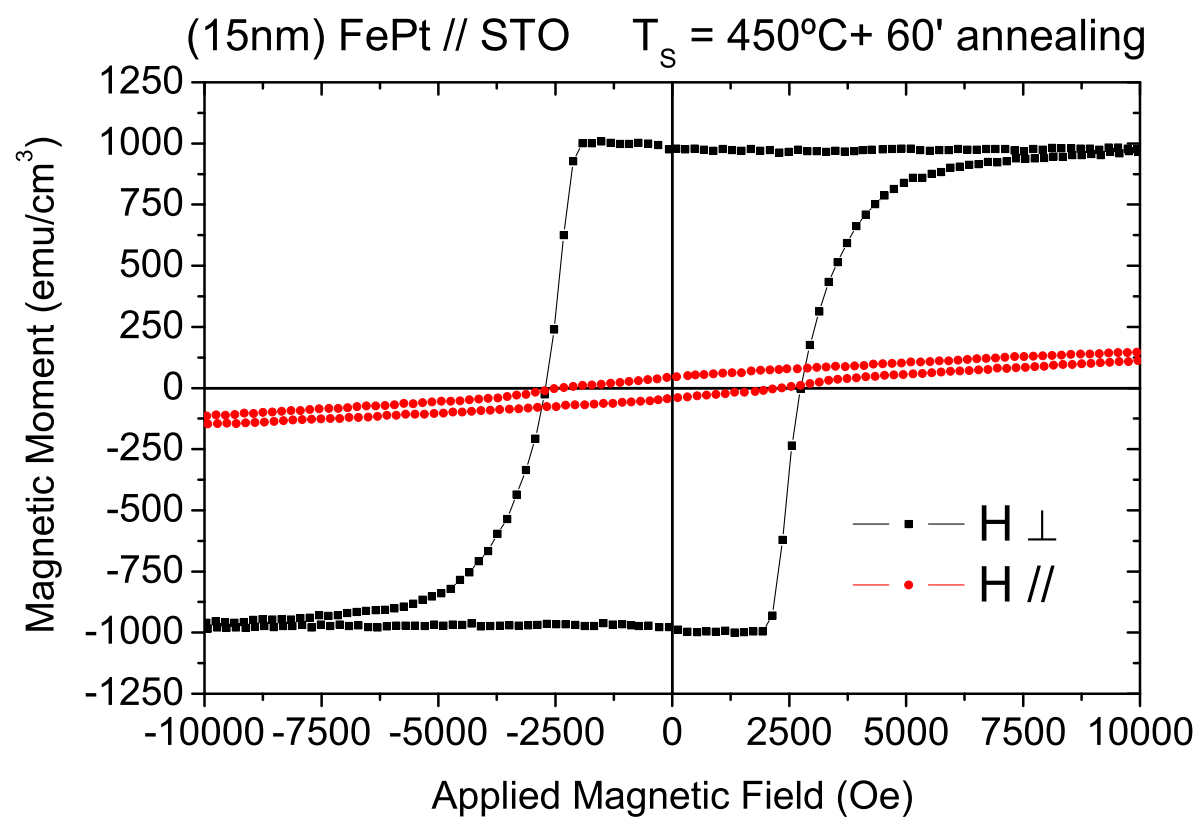

Figure 4.18: Magnetization measurements as a function of the applied magnetic field at room temperature of STO $(001) / /(15 \mathrm{~nm}) \mathrm{FePt}$ film grown at a substrate temperature of $450^{\circ} \mathrm{C}$ and annealed in vacuum for $60^{\prime}$. The magnetic field has been applied in the plane of the film surface (red) and perpendicularly (black).
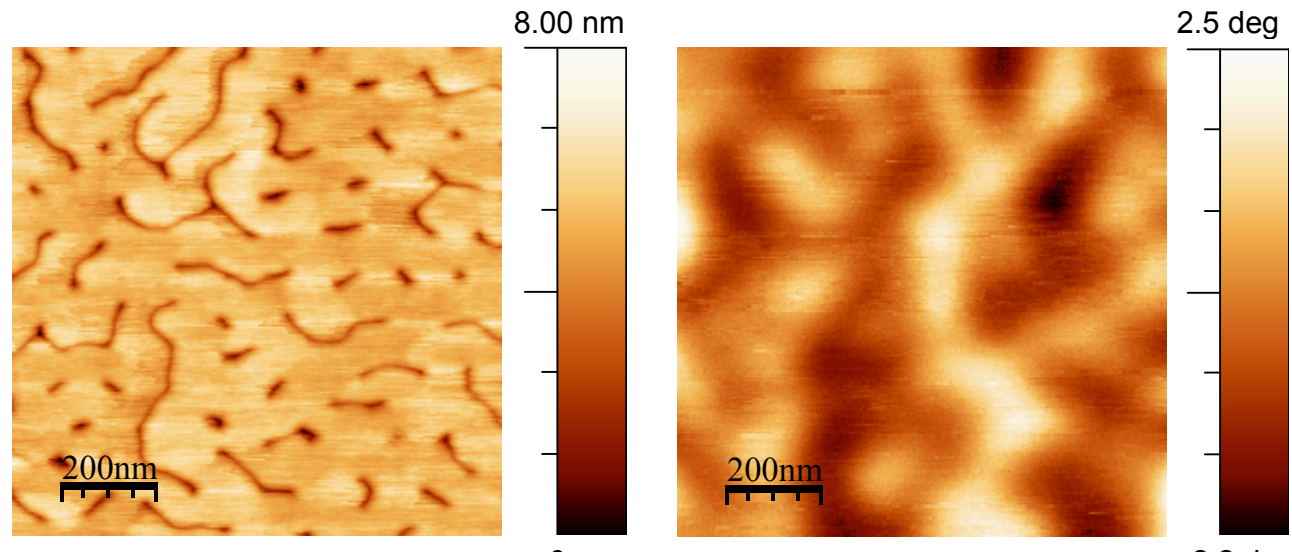

$0 \mathrm{~nm}$

$-2.2 \mathrm{deg}$

Figure 4.19: Left panel: tapping mode AFM image over a region of $1 \mu m \times \mu m$ of the STO // $(15 \mathrm{~nm}) \mathrm{FePt}$ film grown at a substrate temperature of $450^{\circ} \mathrm{C}$ and annealed in vacuum for $60^{\prime}$. Right panel: MFM image obtained simultaneously to the AFM measurement. 
Finally, the sample was structurally characterized by XRD, obtaining a pattern where all the (001) reflections are observed, indicating the epitaxial growth of the $\mathrm{L}_{0}-\mathrm{FePt}$ on the STO (001) and the absence of other crystallographic phases, as the small peaks observed are due to the Al substrate holder of the XRD equipment. Indeed, the $\mathrm{L} 1_{0}-\mathrm{FePt}$ reflections are surrounded by their Laue oscillations, demonstrating the coherence growth of the film (Figure 4.201).

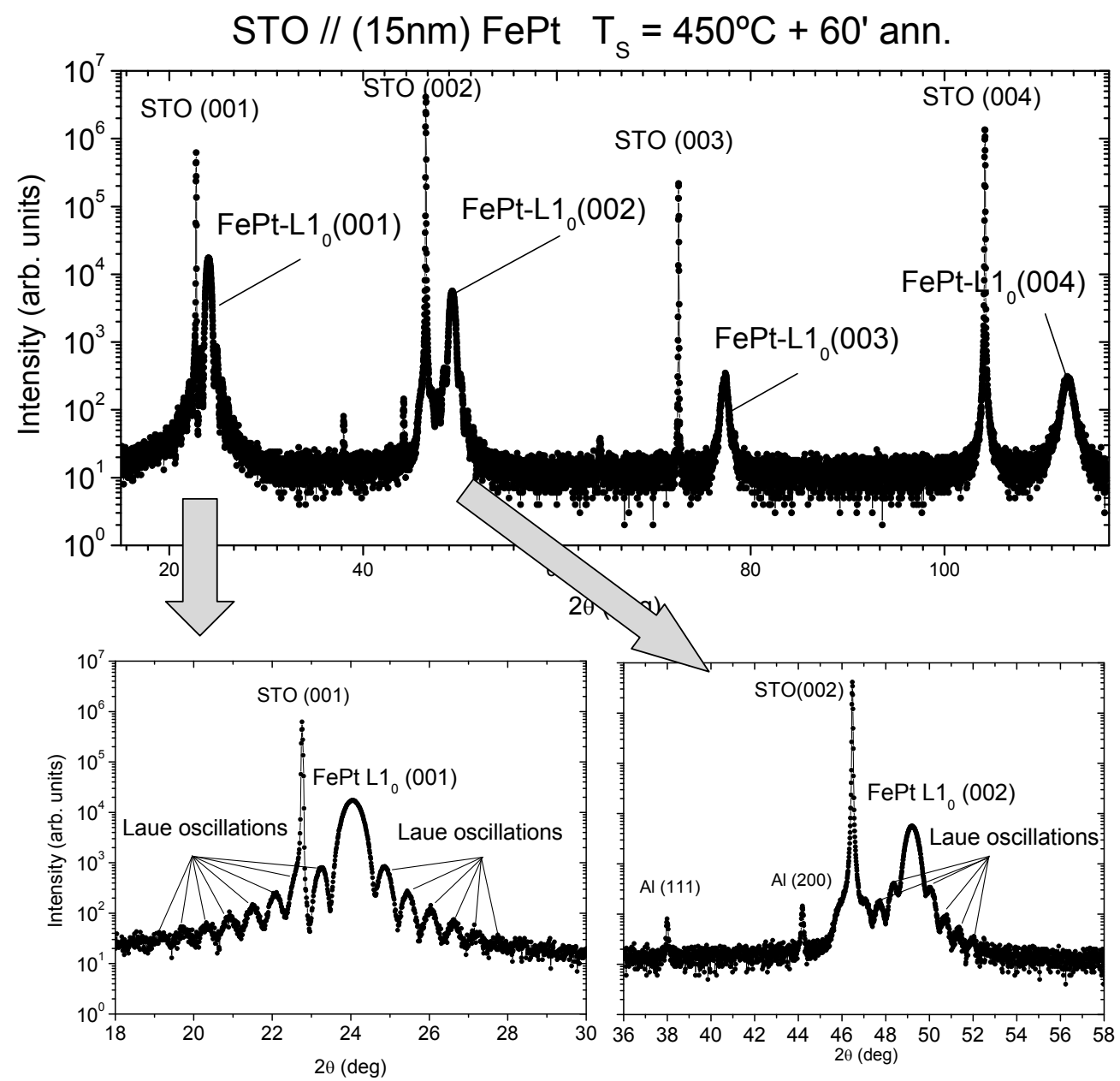

Figure 4.20: XRD pattern of the STO (001) // (15 nm) FePt film grown at $450{ }^{\circ} \mathrm{C}$ and annealed in vacuum for 60 '. 
As a conclusion, we have performed a study about the growth of $15 \mathrm{~nm}$ thick $\mathrm{L} 1_{0}-$ FePt films on different substrates and buffer layers, finding the optimal deposition conditions for $\mathrm{MgO}$ (001) and $\mathrm{SrTiO}_{3}$ (001) substrates, ie, a substrate temperature of $450{ }^{\circ} \mathrm{C}$ for both of them, being necessary an annealing in vacuum for 60 minutes for the growth over STO substrates.

These results will allow us to integrate the FePt films on heterostructures with $\mathrm{MgO}$ tunnel barrier and a magnetite electrode for the future obtention of MTJs, study that is detailed in the next chapter. 



\section{Chapter 5}

\section{$\mathrm{Fe}_{3} \mathrm{O}_{4}$ - based heteroepitaxial structures}

As it was pointed out previously in chapter 2, magnetite is a half-metallic ferromagnet with a high Curie temperature $(860 \mathrm{~K})$ that is expected to exhibit suitable properties for its implementation in spintronic devices [89, 257, 258].

In addition, interest in spintronic structures based on magnetic oxides has increased recently [259], and $\mathrm{MgO}$ is a serious candidate as tunnel barrier because of the small lattice mismatch with the $\mathrm{Fe}_{3} \mathrm{O}_{4}(0.3 \%)$ electrode [260262]. Nevertheless, the performance of $\mathrm{Fe}_{3} \mathrm{O}_{4}$-based MTJs has been limited with scattered values and a maximum of $14 \% \mathrm{MR}$ at room temperature 263 265], and other than $\mathrm{MgO}$ tunnel barriers were not significantly better [266270].

Based on the large spin polarization of the electrons at the Fermi level in magnetite, a heteroestructure with a magnetite electrode should arise significant larger values of magnetoresistance. On these bases and prior to application in real devices, the epitaxial growth of heterostructures with $\mathrm{Fe}_{3} \mathrm{O}_{4}$ and $\mathrm{MgO}$ should be further optimized.

In this chapter the growth and structural and magnetic characterization of heteroepitaxial structures is reported, with at least one magnetite electrode and $\mathrm{MgO}$ barrier. In section 5.1, the growth of $\mathrm{Fe}_{3} \mathrm{O}_{4} / \mathrm{MgO} / \mathrm{Fe}_{3} \mathrm{O}_{4}$ heterostructures is studied, demonstrating the obtention of a high-quality $\mathrm{MgO}$ barrier that is afterwards integrated in $\mathrm{Fe}_{3} \mathrm{O}_{4} / \mathrm{MgO} / \mathrm{Fe}$ (section 5.2) and $\mathrm{Fe}_{3} \mathrm{O}_{4} / \mathrm{MgO} / \mathrm{FePt}$ heterostructures (section 5.3). Optical lithography of these multilayers is currently being performed at the INA to obtain MTJs. 
The $\mathrm{Fe}_{3} \mathrm{O}_{4}$ electrodes have been deposited by PLD, with the optimized growth parameters reported in chapter 2, and electronic transport measurements (magnetoresistance and AHE) in our epitaxial $\mathrm{Fe}_{3} \mathrm{O}_{4}$ thin films have been studied previously (Chapter 2). The other electrodes have been grown by means of different deposition techniques: PLD for Fe deposition, and RF sputtering in the case of the FePt layers.

In section 5.4, preliminary magnetotransport measurements from MTJs with $\mathrm{Fe}_{3} \mathrm{O}_{4}$ bottom electrode are presented. These MTJs were obtained by means of optical lithography from a $\mathrm{MgO}(001) / / \mathrm{Fe}_{3} \mathrm{O}_{4} / \mathrm{MgO} / \mathrm{CoFeB}_{10}$ / $\mathrm{Ru} / \mathrm{Ta}$ heterostructure, whose magnetite electrode was deposited at the INA, and the deposition of the following layers and the lithography process were performed at the INESC-MN in Lisbon (Portugal). 


\section{$5.1 \quad \mathrm{Fe}_{3} \mathrm{O}_{4} / \mathrm{MgO} / \mathrm{Fe}_{3} \mathrm{O}_{4}$}

$\mathrm{Fe}_{3} \mathrm{O}_{4} / \mathrm{MgO} / \mathrm{Fe}_{3} \mathrm{O}_{4}$ heterostructures were grown on $\mathrm{MgO}$ (001) substrates by PLD, all the three layers with the optimized deposition conditions for magnetite detailed in chapter 2 in order to study the crystallinity of the $\mathrm{MgO}$ barrier, i.e. substrate temperature $\mathrm{T}_{S}=450{ }^{\circ} \mathrm{C}$ at a deposition rate of 3.6 $\mathrm{nm} / \mathrm{min}$. in ultra high vacuum.

The thicknesses and roughnesses of the layers were obtained by fitting the XRR pattern by means of the commercial software Leptos (Figure 5.1), demonstrating the relatively low roughness of all the layers forming the heterostructure, specially the upper magnetite electrode (see Table 5.1).

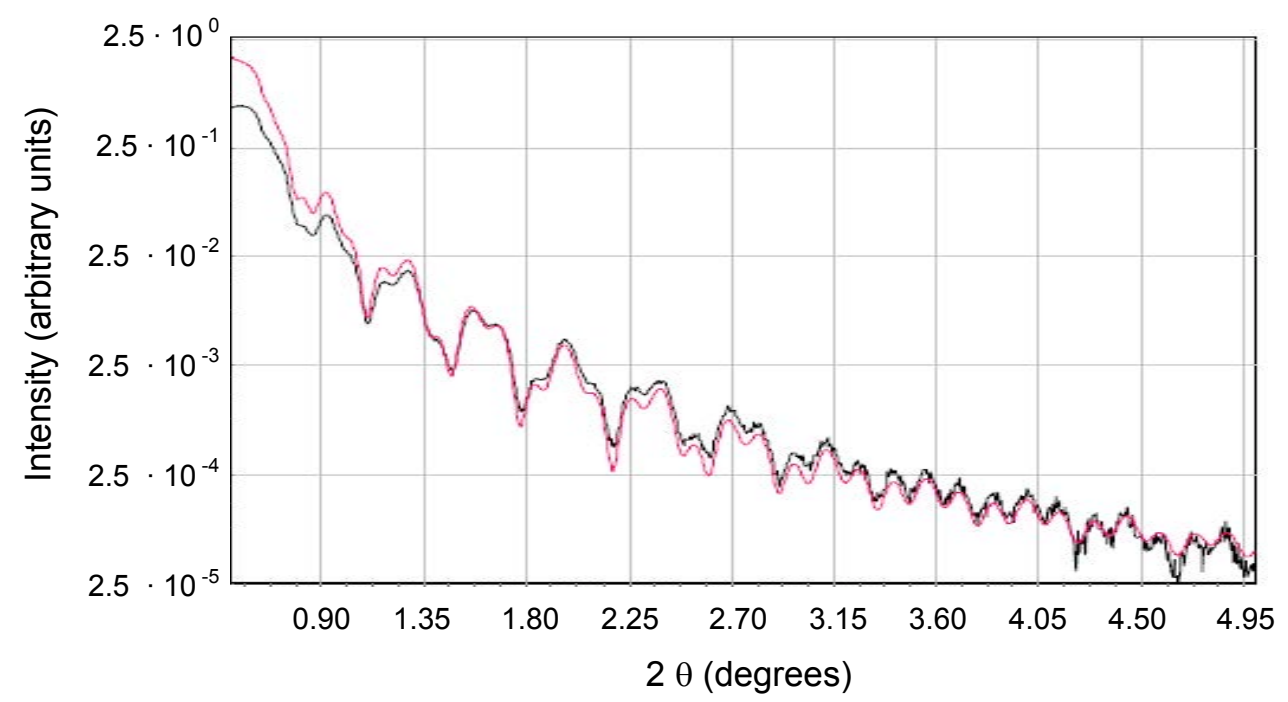

Figure 5.1: XRR measurement from $\mathrm{Fe}_{3} \mathrm{O}_{4} / \mathrm{MgO} / \mathrm{Fe}_{3} \mathrm{O}_{4}$ heterostructure produced at the optimal magnetite growth conditions. The red line is the fit by using the software package LEPTOS from Bruker AXS.

\begin{tabular}{||c|c|c|c||}
\hline \hline Material & Thickness $(\mathrm{nm})$ & Roughness $(\mathrm{nm})$ & Density $\left(\mathrm{g} / \mathrm{cm}^{3}\right)$ \\
\hline $\mathrm{Fe}_{3} \mathrm{O}_{4}$ & 21.3 & 0.01 & 5.24 \\
\hline $\mathrm{MgO}$ & 2.4 & 0.41 & 3.57 \\
\hline $\mathrm{Fe}_{3} \mathrm{O}_{4}$ & 35.7 & 0.62 & 5.24 \\
\hline $\mathrm{MgO}$ & Substrate & 0.35 & 3.57 \\
\hline \hline
\end{tabular}

Table 5.1: Values obtained from the XRR numerical fit in figure 5.1 
The XRD pattern shows the (002) reflection from magnetite and no other crystalline phases are observed (Figure 5.2). The existence of Laue oscillations due to both layers of magnetite indicates the good crystallinity of the upper magnetite film, and therefore, the good crystallinity of the $\mathrm{MgO}$ layer.

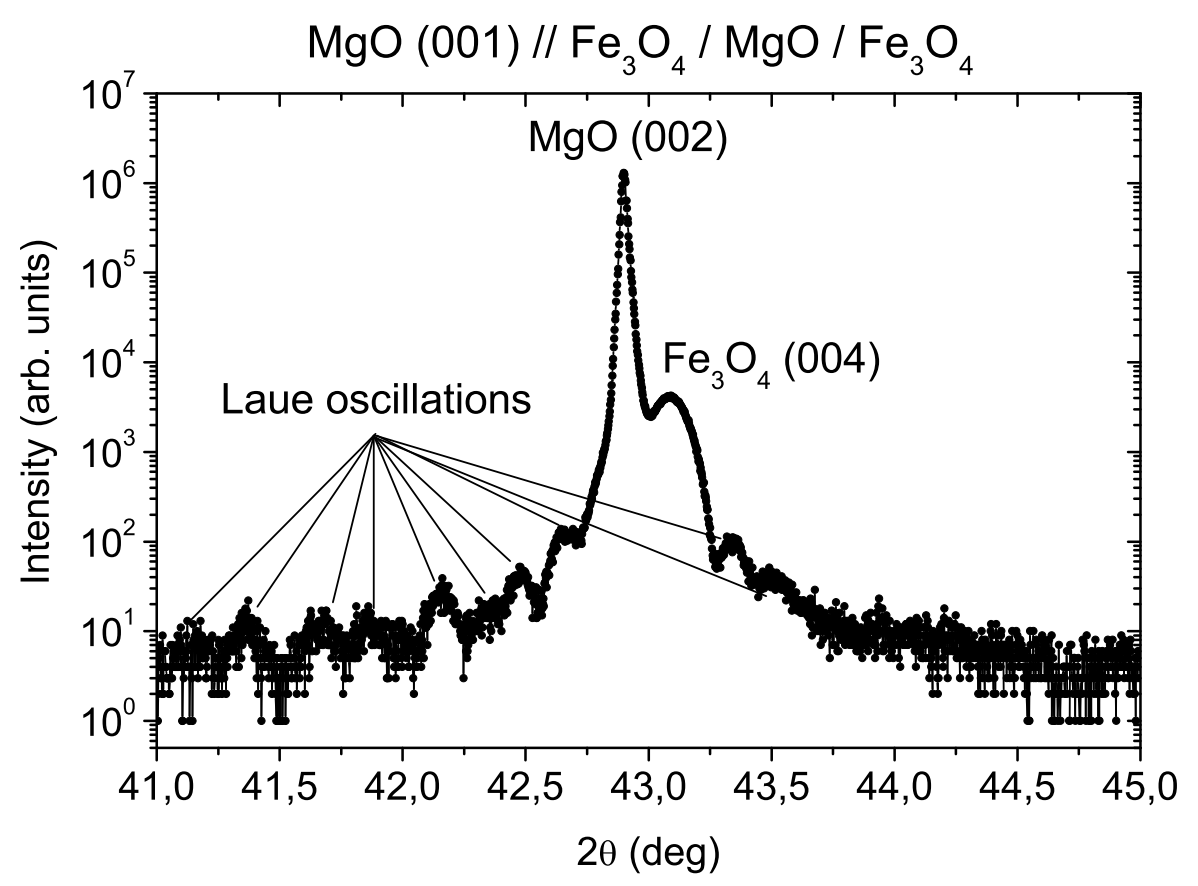

Figure 5.2: $\theta-2 \theta$ scan from a $\mathrm{Fe}_{3} \mathrm{O}_{4} / \mathrm{MgO} / \mathrm{Fe}_{3} \mathrm{O}_{4}$ heterostructure produced at the optimal magnetite growth conditions.

The magnetization measurement as a function of the magnetic field at room temperature up to a maximum magnetic field of 1.8 Tesla was performed by means of VSM (see Figure 5.3). The magnetic moment per volume has been calculated with the total thickness of both $\mathrm{Fe}_{3} \mathrm{O}_{4}$ layers as determined by XRR. The saturation magnetization value is similar to that obtained for magnetite single layers, demonstrating the stoichiometry of the magnetite layers.

As a conclusion, structural and magnetic characterization clearly evidences an epitaxial growth of the $\mathrm{MgO}$ (001) tunnel barrier over the magnetite electrode. This will be further investigated by HRTEM in $\mathrm{Fe}_{3} \mathrm{O}_{4} / \mathrm{MgO} / \mathrm{Fe}$ epitaxial heterostructures in next section. 


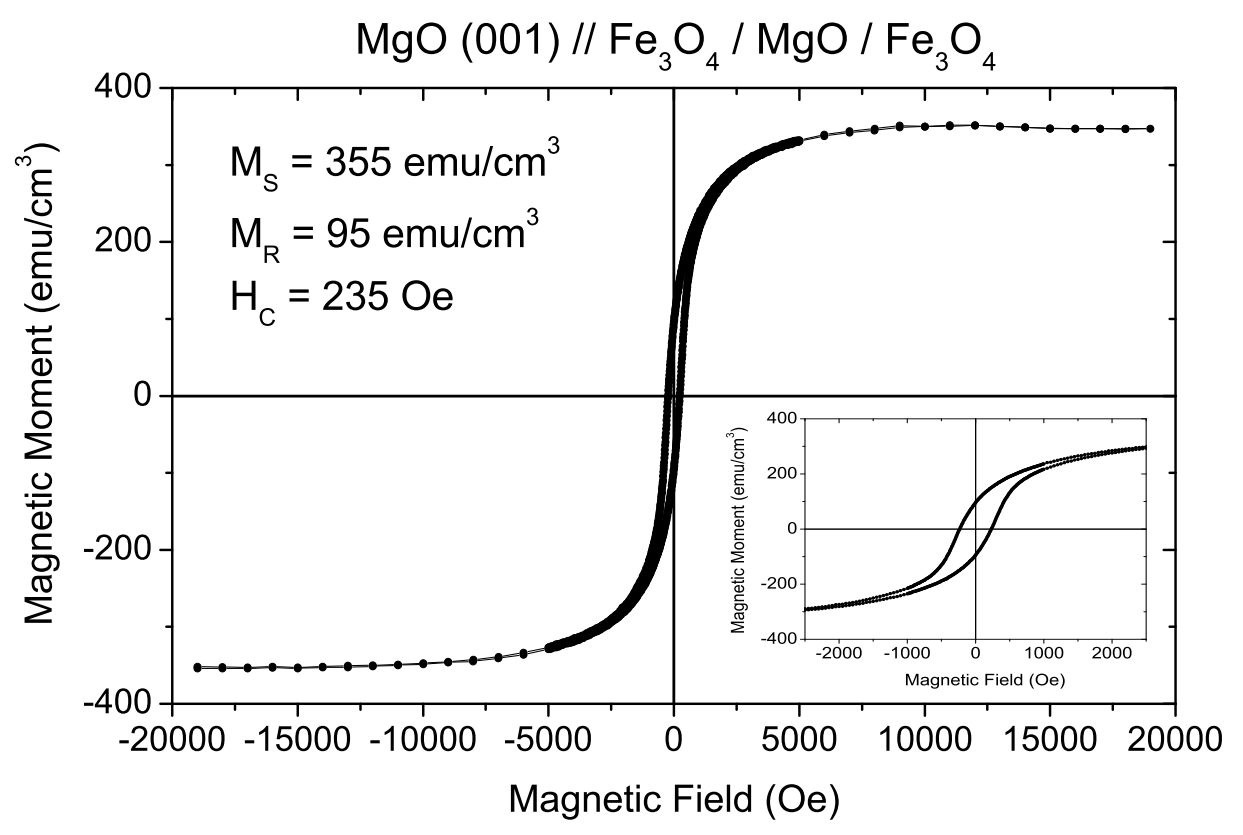

Figure 5.3: Magnetization as a function of the applied magnetic field at room temperature of a $\mathrm{Fe}_{3} \mathrm{O}_{4} / \mathrm{MgO} / \mathrm{Fe}_{3} \mathrm{O}_{4}$ heterostructure produced at the optimal magnetite growth conditions. 


\section{$5.2 \mathrm{Fe}_{3} \mathrm{O}_{4} / \mathrm{MgO} / \mathrm{Fe}$}

In order to grow $\mathrm{Fe}_{3} \mathrm{O}_{4} / \mathrm{MgO} / \mathrm{Fe}$ heterostructures, the growth of Fe epitaxial thin films on single-crystal $\mathrm{MgO}$ (001) substrates was also optimized. The Fe films were deposited in ultra high vacuum by means of PLD using a KrF laser $(248 \mathrm{~nm})$ at $10-\mathrm{Hz}$ repetition rate and pure (99.99\%) Fe targets.

X-ray and HRTEM characterization revealed the expected out-of-plane (001) orientation with a 45 rotation of the in-plane axes, thus:

$$
\mathrm{MgO}(001)[100] / / \mathrm{Fe}(001)[110]
$$

The mosaic spread is typically $\Delta \omega=0.2^{\circ}$ and XRR fits yield an RMS roughness of $0.2 \mathrm{~nm}$ for a Fe typical thickness of $10 \mathrm{~nm}$ and growth temperature of $300{ }^{\circ} \mathrm{C}$. The RT resistivity values are $15.1 \mu \Omega \cdot \mathrm{cm}$ with a residual resistivity ratio $(\mathrm{RRR})$ of $\mathrm{RRR}=4.7$. Magnetization measurements show the expected in-plane magnetic anisotropy with the easy axis along the Fe [001] direction and a coercive field $<10$ Oe (figure 5.4). All these are in good agreement with published data in thin Fe films of similar thickness grown by means of MBE [270] or UHV sputtering [271].

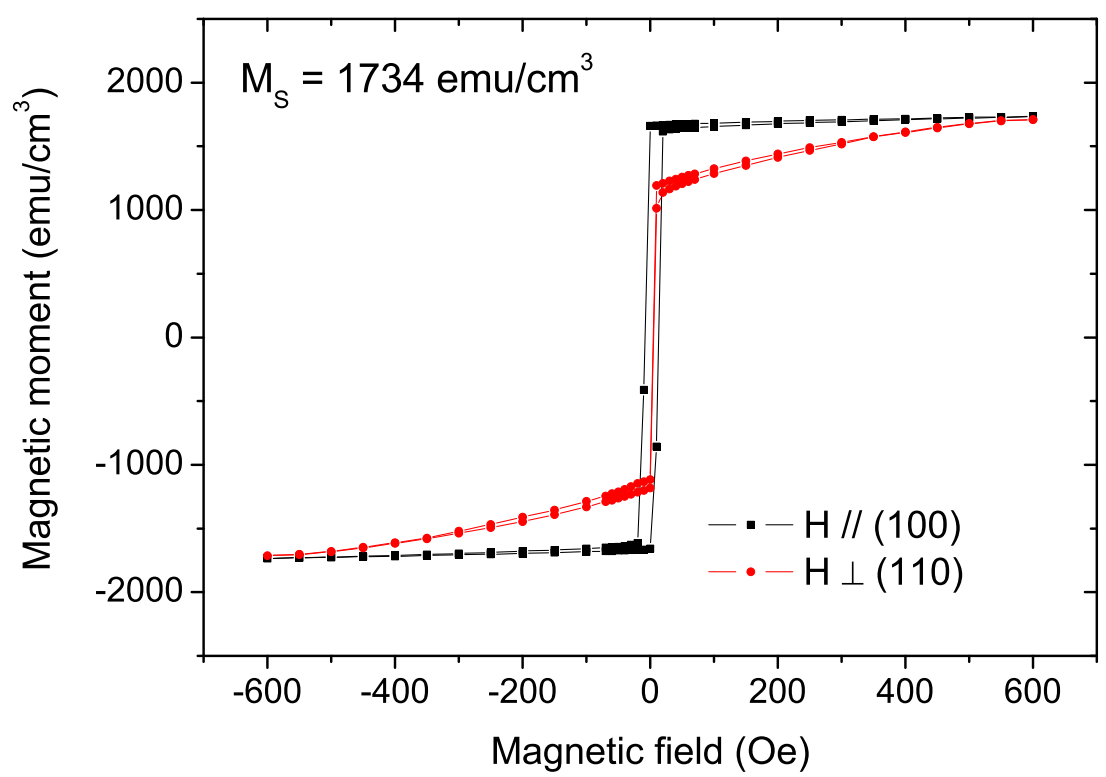

Figure 5.4: Magnetization as a function of the applied magnetic field at room temperature of a $20 \mathrm{~nm}$-thick Fe film. The magnetic field has been applied in the plane of the film, along the (100) and (110) directions. 
All layers of the $\mathrm{Fe}_{3} \mathrm{O}_{4} / \mathrm{MgO} / \mathrm{Fe}$ heterostructure were deposited in ultrahigh vacuum at substrate temperatures of $450{ }^{\circ} \mathrm{C}$ for $\mathrm{Fe}_{3} \mathrm{O}_{4}$ and between room temperature (RT) and $400{ }^{\circ} \mathrm{C}$ for $\mathrm{Fe}$ and $\mathrm{MgO}$ layers. The samples were characterized by x-ray diffraction $(\theta-2 \theta, \omega$ scans, $\phi$ scans, and reciprocal space maps) and XRR, HRTEM, and VSM and SQUID magnetometry.

Cross-section TEM specimens were prepared by mechanical thinning and ion polishing in a commercial Precision Ion Polishing System (PIPS). HRTEM cross-section images obtained in a Philips CM30 $\mathrm{LaB}_{6}$ microscope with a point resolution of $0.19 \mathrm{~nm}$ in selected $\mathrm{Fe}_{3} \mathrm{O}_{4} / \mathrm{MgO} / \mathrm{Fe}$ heterostructures demonstrate the high crystallinity of the epitaxial $\mathrm{MgO}$ (001) tunnel barrier and sharp interfaces (Fig. 5.5).

Combining High Resolution Transmission Electron Microscopy and x-ray results, we have obtained for the heterostructure the epitaxy relation:

$$
\mathrm{MgO}(001)[100] / / \mathrm{Fe}_{3} \mathrm{O}_{4}(001)[100] / \mathrm{MgO}(001)[100] / \mathrm{Fe}(001)[110]
$$

The Fe layer accommodates the strain coming from the large lattice misfit with $\mathrm{MgO}$ barrier $(-3.7 \%)$ by nucleation of dislocations near the interface with the $\mathrm{MgO}$ barrier, one of them is marked with a circle in Fig. 5.5. A 2-nm-thick passivation layer on the Fe surface (labeled as $\mathrm{FeO}_{x}$ ) due to the exposure to ambient atmosphere is observed.

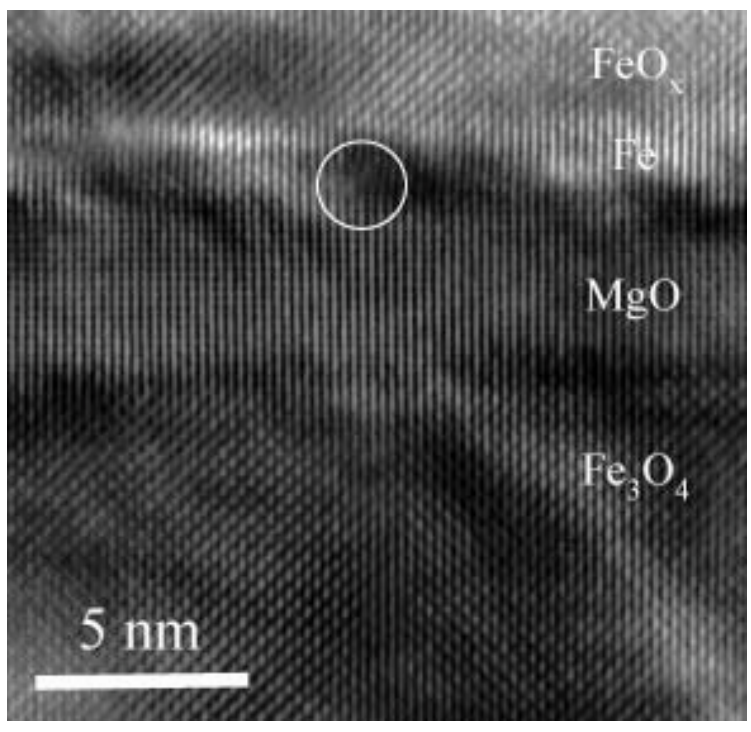

Figure 5.5: Cross-section HRTEM image of a selected $\mathrm{MgO}(001) / / \mathrm{Fe}_{3} \mathrm{O}_{4} / \mathrm{MgO} / \mathrm{Fe}$ epitaxial heterostructure. Image obtained by Dr. C. Magen at the Centre d'Elaboration de Matériaux et d'Etudes Structurales, CNRS in Toulouse (France).

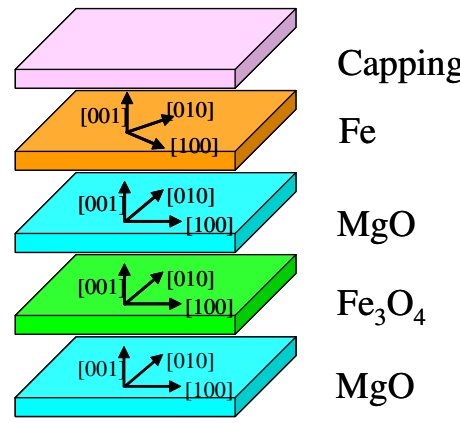

Figure 5.6: Schematic of the heterostructure epitaxial growth. 
The interface roughness and roughness correlation between interfaces in tunnel devices are thought to be critical for their spin-transport properties [262]. XRR in a selected heterostructure where both $\mathrm{MgO}$ barrier and $\mathrm{Fe}$ counterelectrode have been deposited at RT is shown in Fig. 5.7. The top Fe electrode was protected by means of an 8-nm $\mathrm{MgO}$ layer.

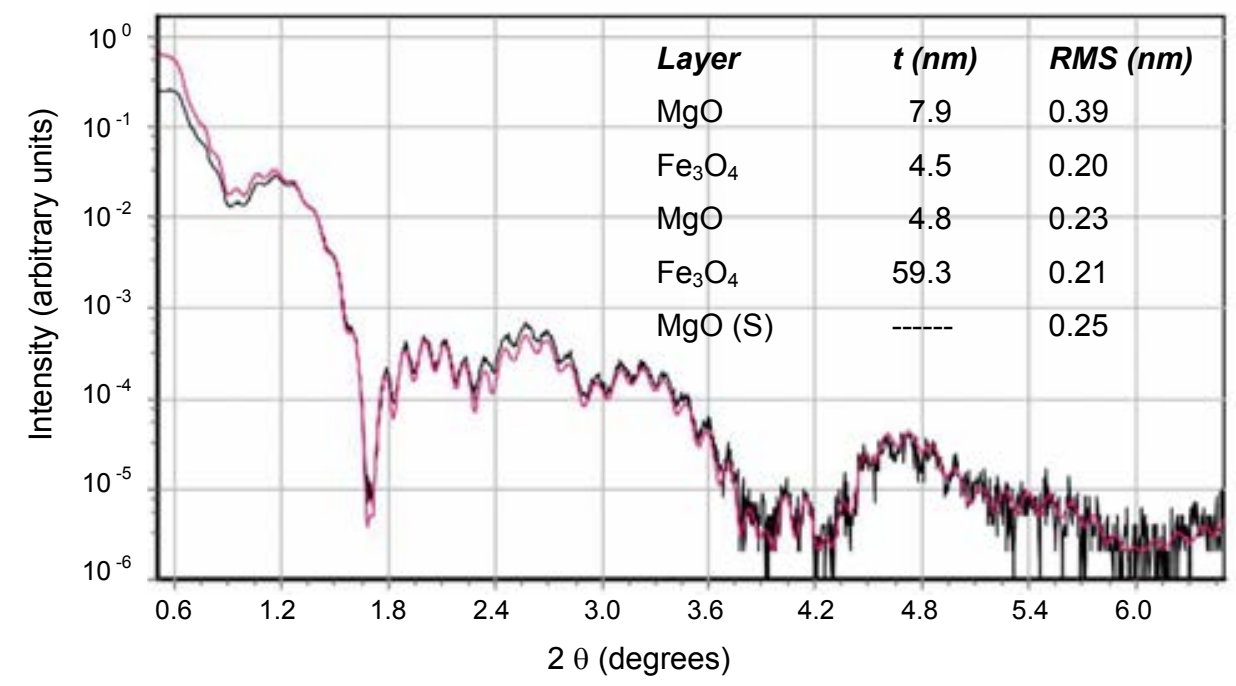

Figure 5.7: XRR of a selected $\mathrm{MgO}(001) / / \mathrm{Fe}_{3} \mathrm{O}_{4} / \mathrm{MgO} / \mathrm{Fe}$ heterostructure. The red line is the fit by using the software package LEPTOS from Bruker AXS. The derived thickness ( $\mathrm{t}$ ) and RMS roughness of all layers and interlayers have been listed as an inset.

As can be seen from the fit, the RMS roughness is relatively low, $\sim 0.2 \mathrm{~nm}$, this being indispensable for the patterning of micrometric MTJs. The obtained values are smaller than those reported in MBE-deposited samples [262], but probably due to the larger roughness of the commercial $\mathrm{MgO}$ substrate utilized in that work and/or different growth mechanisms. In our study, we have found no clear correlation between the interface roughness and the temperature growth of the different layers.

Magnetic characterization of a selected trilayer $(70.9 \mathrm{~nm}) \mathrm{Fe}_{3} \mathrm{O}_{4} /(3.7 \mathrm{~nm})$ $\mathrm{MgO} /(5.6 \mathrm{~nm}) \mathrm{Fe}$ protected by $5.3 \mathrm{~nm}$ of $\mathrm{Au}$ (sputter-deposited in the same vacuum chamber) was performed by means of VSM and SQUID magnetometry.

The RT hysteresis loop was measured at room temperature up to a maximum applied field of 1.8 Tesla by VSM magnetometry. The magnetic field was applied in the (110) $\mathrm{MgO}$ direction, thus, in the magnetic easy axis of the Fe layer (Fig. 5.8). It reveals an independent switching of both $\mathrm{Fe}_{3} \mathrm{O}_{4}$ and $\mathrm{Fe}$ electrodes, which is essential for the existence of tunnel magnetoresistance. 
The switching fields of both $\mathrm{Fe}_{3} \mathrm{O}_{4}$ (200 Oe) and $\mathrm{Fe}$ (46 Oe) electrodes are smaller and larger, respectively, than those typically observed in single layers on $\mathrm{MgO}$ (001). This indicates the existence of a weak ferromagnetic coupling between both electrodes. We have estimated the exchange coupling constant, $J$, between electrodes by either measuring a major hysteresis loop or by measuring a minor hysteresis loop of the Fe layer with smallest coercive field. We have obtained values of $J=0.03 \mathrm{~mJ} / \mathrm{m}^{2}$ for samples with an $\mathrm{MgO}$ tunnel barrier thickness of $2 \mathrm{~nm}$, this being consistent with published data [272], and thus confirming the required weak coupling for future MTJs.

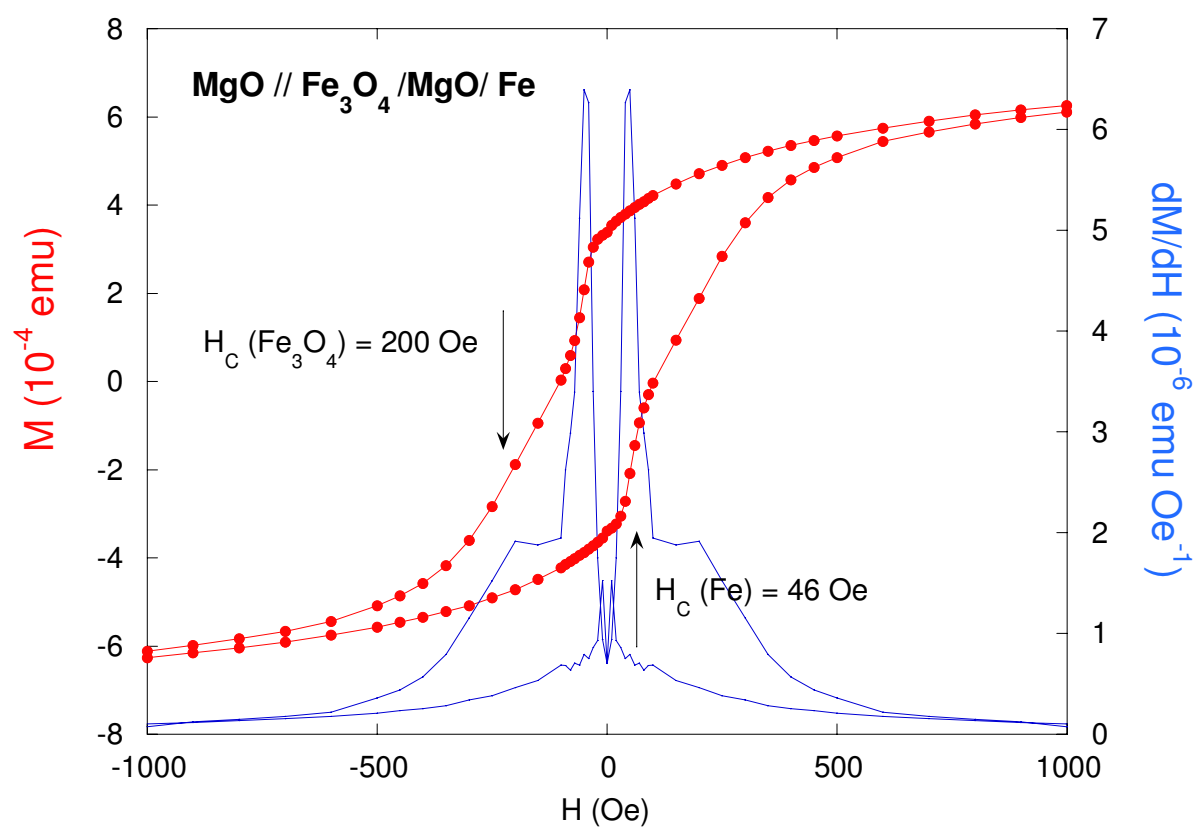

Figure 5.8: RT hysteresis loop of a $(70.9 \mathrm{~nm}) \mathrm{Fe}_{3} \mathrm{O}_{4} /(3.7 \mathrm{~nm}) \mathrm{MgO} /(5.6 \mathrm{~nm})$ $\mathrm{Fe} /(5.3 \mathrm{~nm}) \mathrm{Au}$ heterostructure. The switching fields of both electrodes have been marked.

The low-field temperature dependence of the magnetization of the same sample was performed by means of SQUID magnetometry at a magnetic field of 500 Oe, applied in the $\mathrm{MgO}$ (100) direction (Fig. 5.9). A clear and sharp drop at the Verwey transition, $\mathrm{T}_{V}=115 \mathrm{~K}$, can be observed, demonstrating that the high quality of the $\mathrm{Fe}_{3} \mathrm{O}_{4}$ layer is preserved after the subsequent growth of the $\mathrm{MgO}, \mathrm{Fe}$ and capping layers, this guaranteeing a source of nearly fully polarized electrons. 


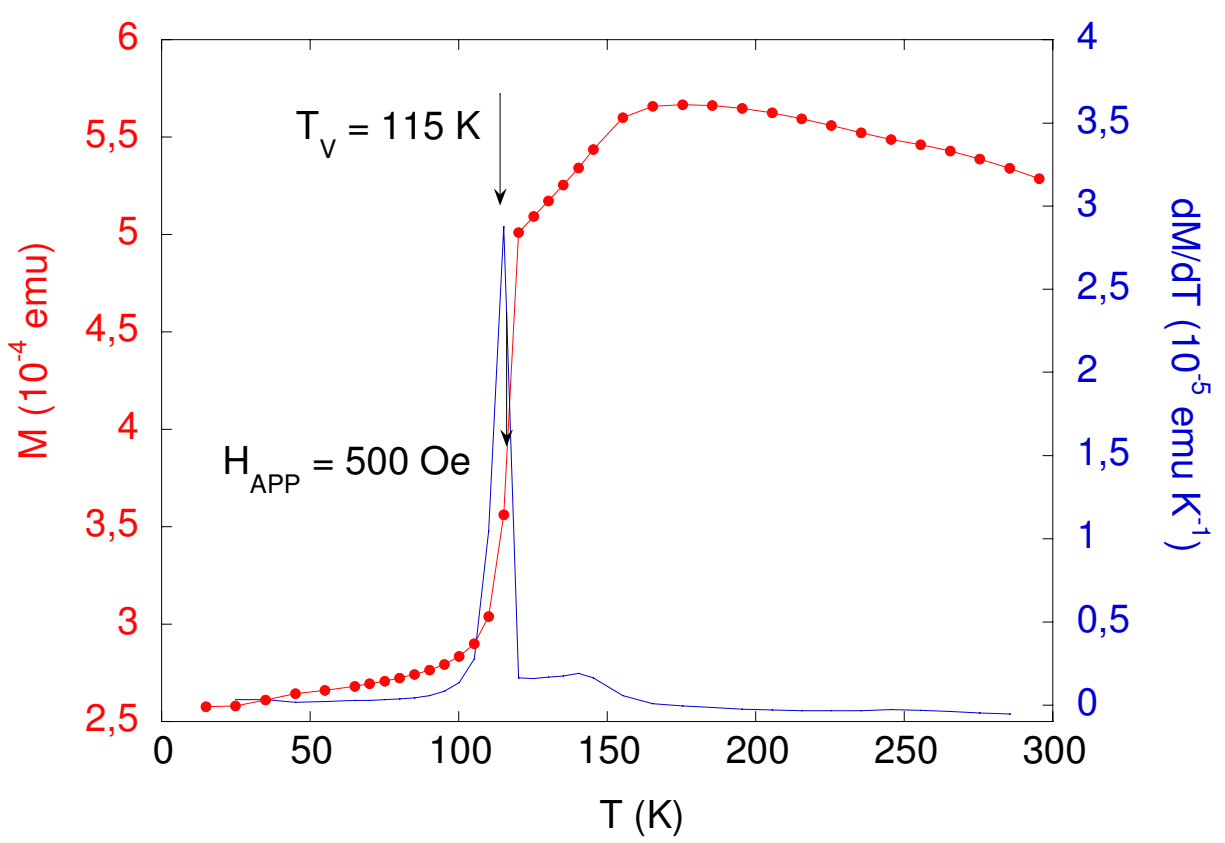

Figure 5.9: Temperature dependence of the magnetization in a field of 500 Oe of the $(70.9 \mathrm{~nm}) \mathrm{Fe}_{3} \mathrm{O}_{4} /(3.7 \mathrm{~nm}) \mathrm{MgO} /(5.6 \mathrm{~nm}) \mathrm{Fe} /(5.3 \mathrm{~nm}) \mathrm{Au}$ heterostructure. The onset of the Verwey transition is indicated.

In conclusion, the structural, morphological and magnetic characterization of the $\mathrm{Fe}_{3} \mathrm{O}_{4} / \mathrm{MgO} / \mathrm{Fe}$ heterostructures demonstrates the high crystallinity and clean interfaces required for further research and applications of their magnetotransport properties [273]. The good control of the tunnel barrier thickness has allowed a satisfactory decoupling of the ferromagnetic layers, which is essential for large tunnel magnetoresistance. 


\section{$5.3 \mathrm{Fe}_{3} \mathrm{O}_{4} / \mathrm{MgO} / \mathrm{FePt}$}

As it has been pointed out previously, magnetite is expected to exhibit suitable properties for its implementation in spintronic devices (see chapter 2), and $L 1_{0}$ - ordered FePt films present a high magnetocrystalline anisotropy energy density with the easy magnetization direction perpendicular to the film surface plane, which solves the superparamagnetic limit problem (chapter 4). Therefore, MTJs composed by $\mathrm{Fe}_{3} \mathrm{O}_{4} / \mathrm{MgO} / \mathrm{FePt}$ heterostructures are promising devices in spintronics.

FePt has shown high tunnel magnetoresistance when being part of a MTJ with $\mathrm{MgO}(001)$ barrier [46, 50, 51]. However, both electrodes in those MTJs have the easy magnetization direction perpendicular to the film surface, and TMR has never been examined in any MTJ including both in-plane and outof-plane anisotropy geometries in MTJs electrodes. Obtaining high quality $\mathrm{Fe}_{3} \mathrm{O}_{4} / \mathrm{MgO} / \mathrm{FePt}$ represents, then, an interesting and timely topic.

Considering that the growth of magnetite and FePt films was previously optimized (chapters 2 and 4, respectively), there are two possible heterostructure geometries that are suitable for the subsequent optical lithography process necessary in order to obtain MTJs:

- $\mathrm{MgO}(001) / / \mathrm{Fe}_{3} \mathrm{O}_{4} / \mathrm{MgO} / \mathrm{FePt}$

In this geometry, the relatively thick conductive bottom electrode necessary for an optimal lithography process is the magnetite film.

- Nb:STO (001) // FePt / $\mathrm{MgO} / \mathrm{Fe}_{3} \mathrm{O}_{4}$

In this case, the conductive $\mathrm{Nb}: S T O$ substrate provides the bottom electrode thickness necessary for the lithography process, allowing the growth of a thinner FePt film. This heterostructure is grown and studied over STO substrates prior to its deposition over the conductive $\mathrm{Nb}$ : $S T O$ substrates, as their structural and morphological characteristics are identical.

Prior to the deposition of the heterostructures with the suitable geometry for lithography, a $\mathrm{MgO}(001) / / \mathrm{FePt} / \mathrm{MgO} / \mathrm{Fe}_{3} \mathrm{O}_{4}$ heterostructure was grown and characterized in order to determine the viability of such heterostructures.

The $\mathrm{MgO}$ and $\mathrm{Fe}_{3} \mathrm{O}_{4}$ layers were grown by means of PLD at the INA (Spain), and the FePt layers were grown by RF-sputtering at the IMEM (Italy). The structural, morphological and magnetic characterization of the heterostructures was performed at the INA (Spain). 


\subsection{1 $\mathrm{MgO}(001) / / \mathrm{FePt} / \mathrm{MgO} / \mathrm{Fe}_{3} \mathrm{O}_{4}$}

A $12 \mathrm{~nm}$-thick FePt film was grown on $\mathrm{MgO}$ (100) by means of RF-sputtering at the optimized conditions detailed in chapter 4. The $\mathrm{MgO} / \mathrm{Fe}_{3} \mathrm{O}_{4}$ structure was grown by PLD, with the deposition conditions detailed in chapter 2 .

The XRR pattern was measured and fitted (figure 5.10), showing relatively low roughnesses for the $\mathrm{Fe}_{3} \mathrm{O}_{4}, \mathrm{FePt}$ and $\mathrm{MgO}$ layers.

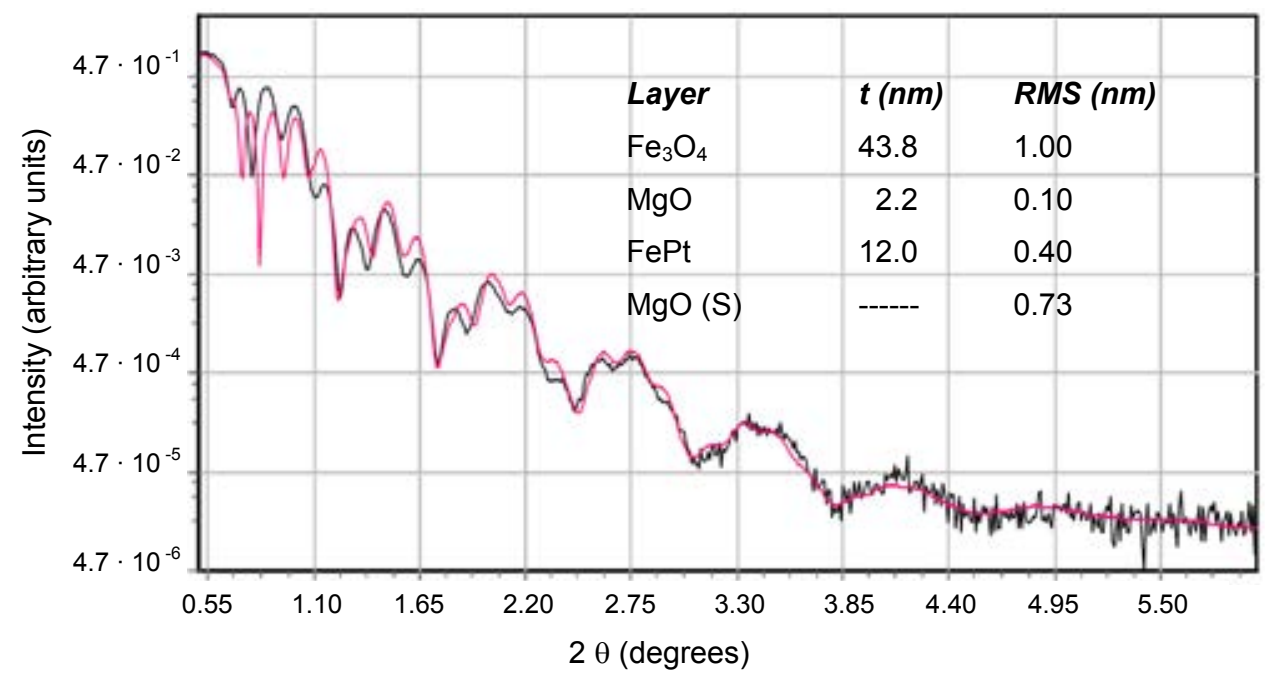

Figure 5.10: XRR of a selected $\mathrm{MgO}(001) / / \mathrm{Fe}_{3} \mathrm{O}_{4} / \mathrm{MgO} / \mathrm{FePt}$ heterostructure. The red line is the fit by using the software package LEPTOS from Bruker AXS. The derived thickness (t) and RMS roughness of all layers and interlayers have been listed as an inset.

The RT hysteresis loop of the heterostructure was measured at room temperature up to a maximum applied field of 1.8 Tesla by VSM magnetometry (see Fig. 5.11). The magnetic field $(\mathrm{H})$ was applied both in the plane of the substrate surface and perpendicular to the surface plane, showing that the easy magnetization axis of the FePt film is perpendicular to the film surface, while the magnetite has its easy magnetization direction in the plane of the surface.

Both the coercive field of the FePt film and the demagnetizing field of the magnetite, $\mathrm{H}_{D}\left(\mathrm{Fe}_{3} \mathrm{O}_{4}\right)=4 \pi \mathrm{M}_{S} \approx 6 \mathrm{kOe}$, determine the shape of the hysteresis loop when $\mathrm{H}$ is applied perpendicularly to the sample surface. 
For high applied magnetic field $\left(\mathrm{H}>\mathrm{H}_{D}\left(\mathrm{Fe}_{3} \mathrm{O}_{4}\right), \mathrm{H}_{C}\right)$ the magnetization direction of both electrodes are oriented parallel to the applied magnetic field (situation (a) in figures 5.11 and 5.12). As $\mathrm{H}$ is decreased, the magnetite magnetization rotates towards its easy magnetization direction in the film plane (b). Further increasing of the magnetic field in the opposite direction rotates the magnetite magnetization to a direction parallel to $\mathrm{H}$ direction (c). When $\mathrm{H}>\mathrm{H}_{D}\left(\mathrm{Fe}_{3} \mathrm{O}_{4}\right)$, the magnetization of the magnetite is parallel to $\mathrm{H}$. When $\mathrm{H}$ overcomes the coercive field of the FePt layer, the total magnetization is parallel to $\mathrm{H}(\mathrm{d})$.

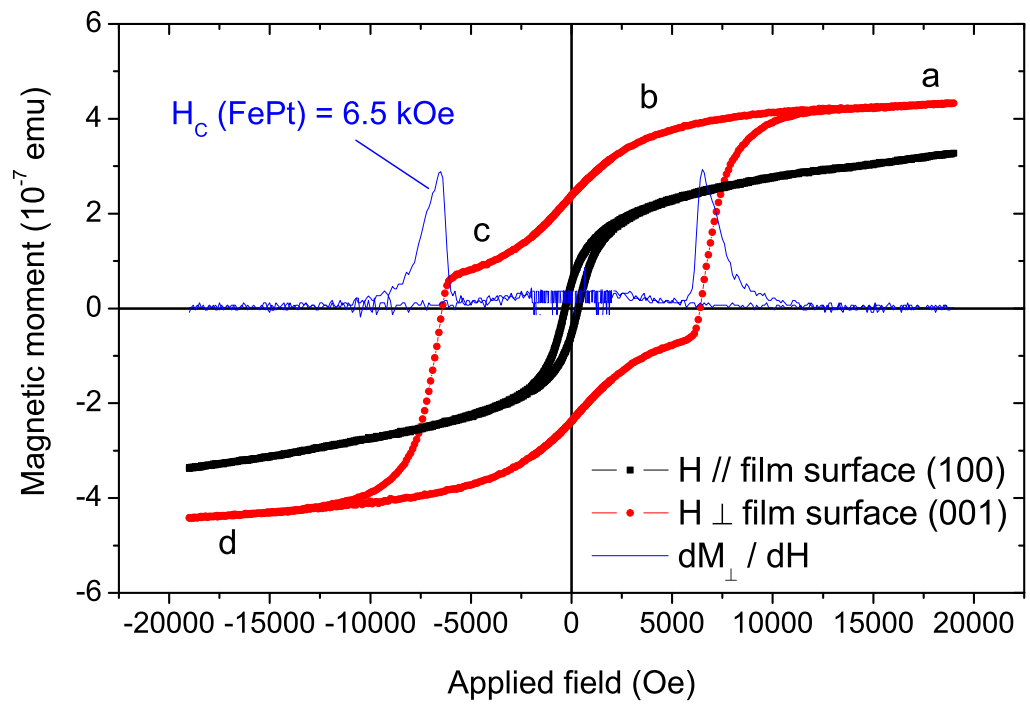

Figure 5.11: RT hysteresis loop of a $\mathrm{MgO}(001) / /(12.0 \mathrm{~nm}) \mathrm{FePt} /(2.2 \mathrm{~nm}) \mathrm{MgO} /$ $(43.8 \mathrm{~nm}) \mathrm{Fe}_{3} \mathrm{O}_{4}$ heterostructure. The coercive field of the FePt film has been marked.

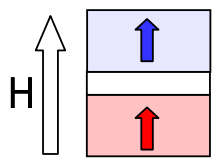

a

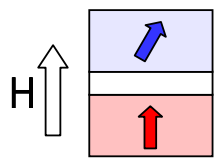

b

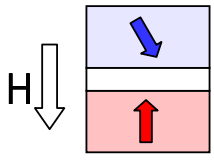

C

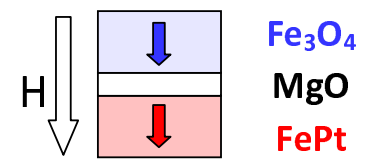

d

Figure 5.12: Schematic of the magnetization orientation of the electrodes in the situations displayed in figure 5.11

It is then demonstrated that the $\mathrm{FePt} / \mathrm{MgO} / \mathrm{Fe}_{3} \mathrm{O}_{4}$ heterostructure can be obtained with an acceptable quality, obtaining a system with an unusual magnetic behavior, which makes it interesting for MTJs obtention. 


\subsection{2 $\mathrm{MgO}(001) / / \mathrm{Fe}_{3} \mathrm{O}_{4} / \mathrm{MgO} / \mathrm{FePt}$}

The $\mathrm{Fe}_{3} \mathrm{O}_{4} / \mathrm{MgO}$ structure was grown on $\mathrm{MgO}$ (001) substrates by PLD, using stoichiometric polycrystalline $\mathrm{Fe}_{3} \mathrm{O}_{4}$ and $\mathrm{MgO}$ targets, and a $\mathrm{KrF}$ laser (248 $\mathrm{nm}$ ) in ultra-high vacuum (base pressure $<5 \times 10^{-9}$ Torr), at substrate temperatures of $400{ }^{\circ} \mathrm{C}$. Afterwards a $10 \mathrm{~nm}$-thick FePt film was grown over the structure using the deposition conditions optimized in chapter 4, which, for FePt film with this thickness on $\mathrm{MgO}$ substrates consist on a substrate temperature of $442{ }^{\circ} \mathrm{C}$ and a posterior annealing in high vacuum for 85 minutes.

The XRR pattern was measured and fitted (figure 5.13), showing low roughness for the $\mathrm{Fe}_{3} \mathrm{O}_{4}$ and $\mathrm{MgO}$ layers, $<0.1 \mathrm{~nm}$, and the expected value for the FePt film surface.

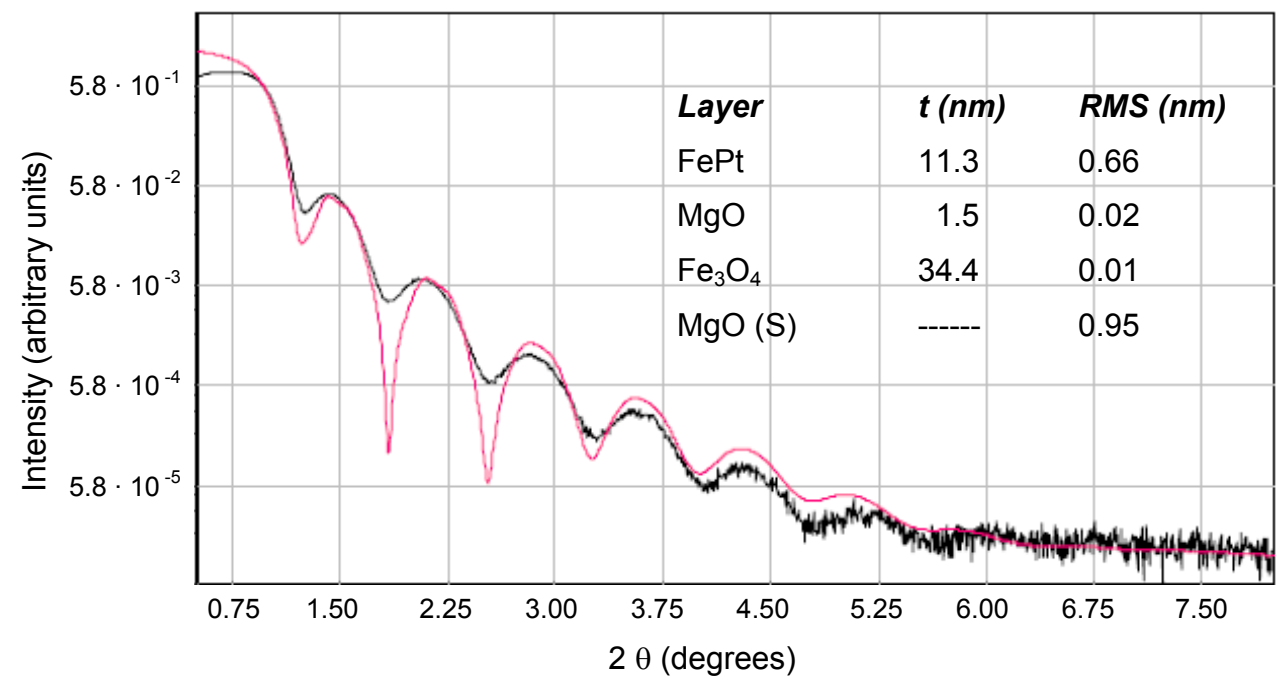

Figure 5.13: XRR of a selected $\mathrm{MgO}(001) / / \mathrm{Fe}_{3} \mathrm{O}_{4} / \mathrm{MgO} / \mathrm{FePt}$ heterostructure. The red line is the fit by using the software package LEPTOS from Bruker AXS. The derived thickness ( $\mathrm{t}$ ) and RMS roughness of all layers and interlayers have been listed as an inset.

The RT hysteresis loop of the previous heterostructure was measured at room temperature up to a maximum applied field of 1.8 Tesla by VSM magnetometry. The magnetic field was applied both in the plane of the substrate surface and perpendicular to the surface plane, showing that the expected easy magnetization direction for the FePt and the magnetite, being perpendicular and parallel to the sample surface, respectively. The behavior of the magnetization directions of both electrodes as a function of the applied magnetic field corresponds to the situations explained previously in section 5.3.1. 
In this case, from the hysteresis loop obtained by applying the magnetic field perpendicular to the film surface, it can be observed that the coercive field of the FePt film $\left(\mathrm{H}_{C}(\mathrm{FePt})=2.6 \mathrm{kOe}\right)$ is lower than the magnetite demagnetizing field $\left(\mathrm{H}_{D}\left(\mathrm{Fe}_{3} \mathrm{O}_{4}\right)=5 \mathrm{kOe}\right)$ for a saturation magnetization of 400 $\left.\mathrm{emu} / \mathrm{cm}^{3}\right)$ ), which can be noticed by means of the derivative curve. Such decrease is possibly mainly due to the epitaxial strain present in the system, which slightly modifies the in plane lattice parameter of the deposited $\mathrm{MgO}$ substrate, leading to a change in the morphology of the subsequently grown FePt film.

The hysteresis loop measured with the magnetic field applied parallel to the sample surface shows a superposition of the slope due to the FePt contribution, and the signal of the magnetite film, showing a coercive field whose value is similar to those measured in magnetite thin films.

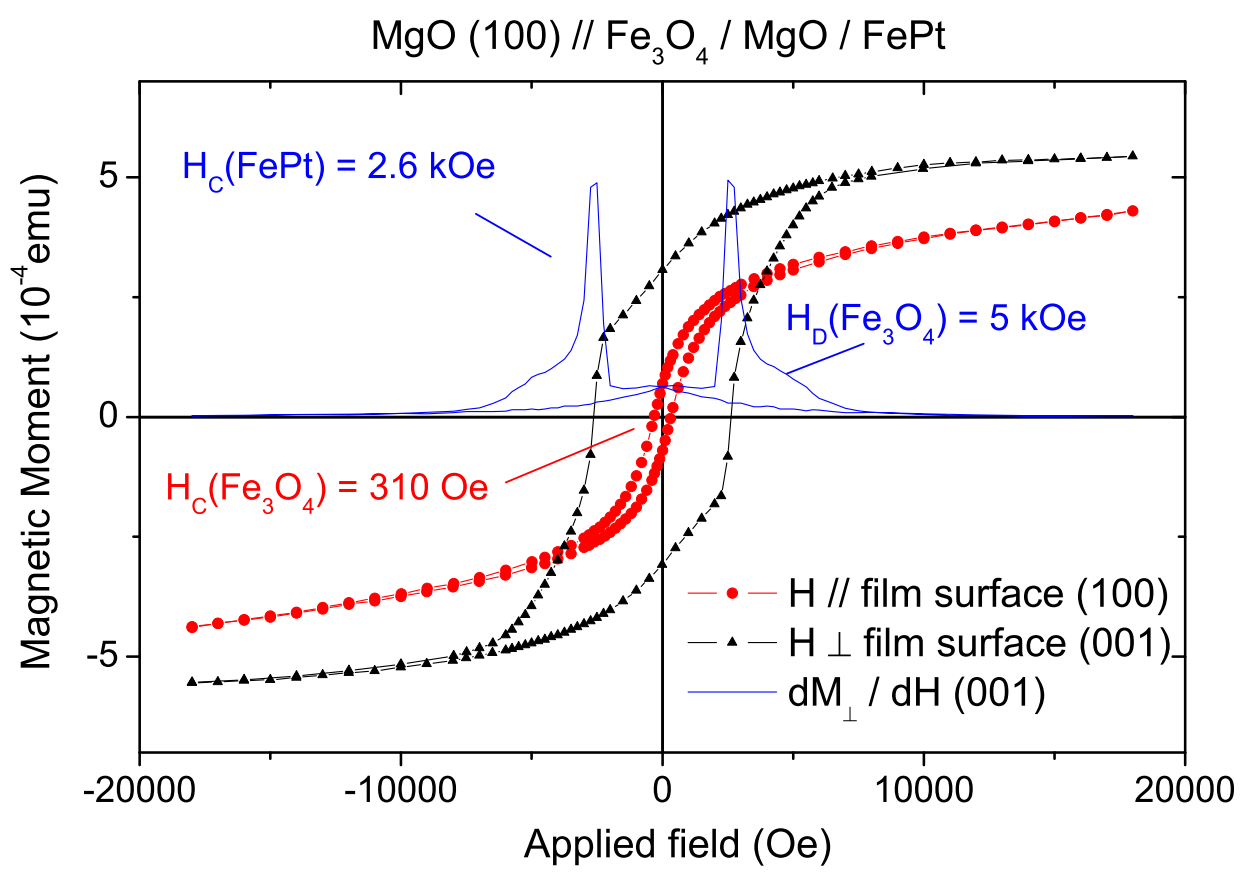

Figure 5.14: RT hysteresis loop of a $\mathrm{MgO}(001) / /(34.4 \mathrm{~nm}) \mathrm{Fe}_{3} \mathrm{O}_{4} /(1.5 \mathrm{~nm}) \mathrm{MgO}$ / (11.3 nm) FePt heterostructure. 
The XRD pattern displayed in figure 5.15 shows the [001] reflections from the $\mathrm{L} 1_{0}$ - ordered FePt, which, together with the magnetization measurements, demonstrates the good crystallinity and epitaxial growth of the $\mathrm{L} 1_{0}-\mathrm{FePt}$ (001) ordered phase. As the $\mathrm{Fe}_{3} \mathrm{O}_{4}$ (004) reflection is also visible, it is evidenced that the magnetite bottom layer quality is not modified during the growth of the rest of the layers.

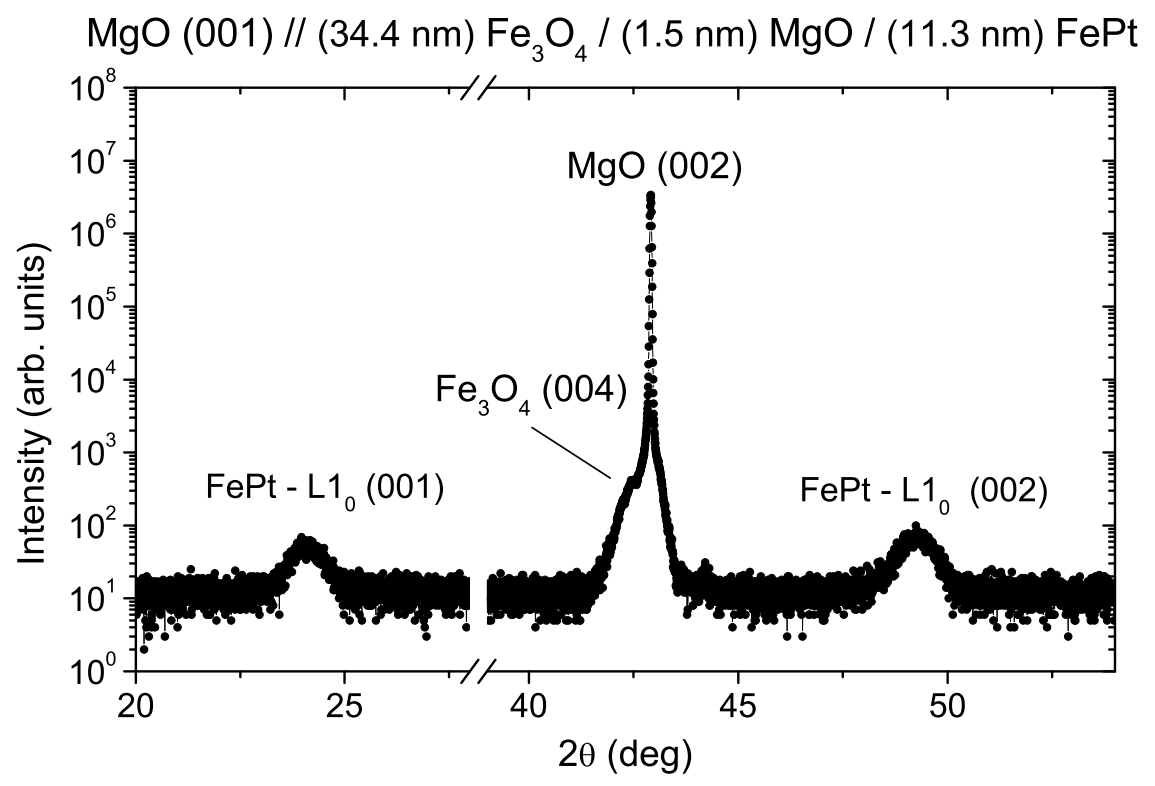

Figure 5.15: $\theta-2 \theta$ scan from a $\mathrm{MgO}(001) / /(34.4 \mathrm{~nm}) \mathrm{Fe}_{3} \mathrm{O}_{4} /(1.5 \mathrm{~nm}) \mathrm{MgO} /$ $(11.3 \mathrm{~nm})$ FePt heterostructure.

The growth of $\mathrm{MgO}(001) / /(\sim 40 \mathrm{~nm}) \mathrm{Fe}_{3} \mathrm{O}_{4} /(\sim 2 \mathrm{~nm}) \mathrm{MgO} /(10 \mathrm{~nm})$ FePt is, then, fully optimized, demonstrating its high crystalline quality and its promising magnetic properties. Optical lithography of this heterostructure is now in progress in order to obtain MTJs. 


\subsection{3 $\mathrm{STO}(001) / / \mathrm{FePt} / \mathrm{MgO} / \mathrm{Fe}_{3} \mathrm{O}_{4}$}

A $15 \mathrm{~nm}$-thick FePt film was grown on STO by means of RF-sputtering at the optimized conditions detailed in chapter 4, i.e. substrate temperature of $450^{\circ} \mathrm{C}$ and an in-situ annealing of 60 minutes. The $\mathrm{Fe}_{3} \mathrm{O}_{4} / \mathrm{MgO}$ structure was grown by PLD, with the deposition conditions detailed in chapter 2 ,

The XRR pattern was measured and fitted (figure 5.16), showing considerable roughnesses for the $\mathrm{Fe}_{3} \mathrm{O}_{4}$ and $\mathrm{MgO}$ layers.

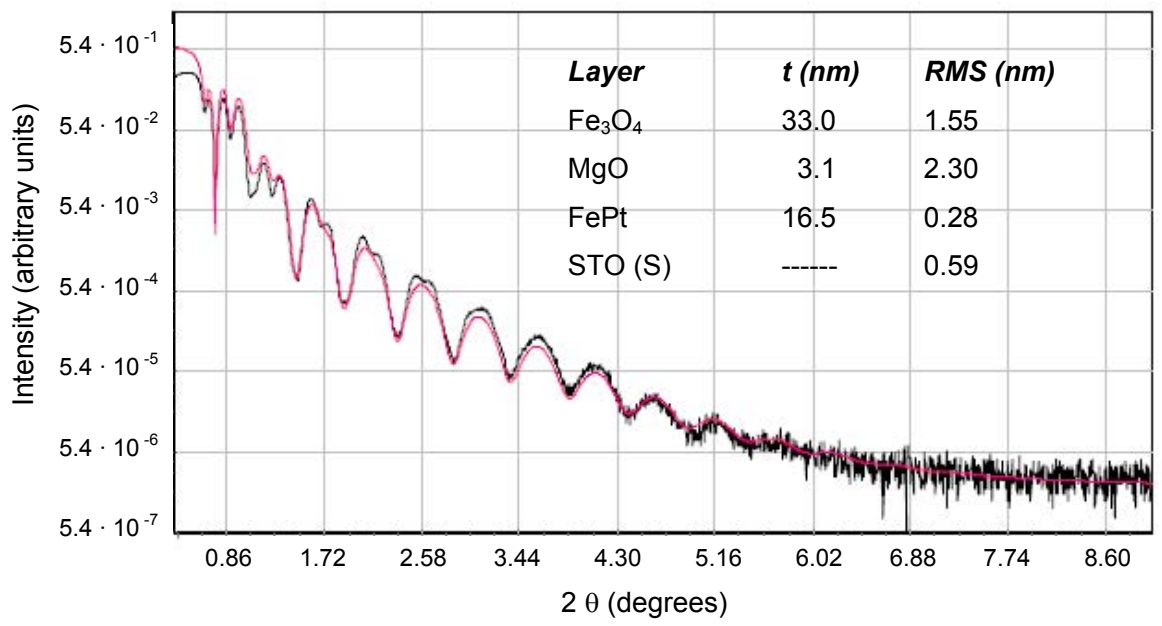

Figure 5.16: XRR of a selected $\mathrm{STO}(001) / / \mathrm{Fe}_{3} \mathrm{O}_{4} / \mathrm{MgO} / \mathrm{FePt}$ heterostructure. The red line is the fit by using the software package LEPTOS from Bruker AXS. The derived thickness (t) and RMS roughness of all layers and interlayers have been listed as an inset.

The RT hysteresis loop of the heterostructure was measured at room temperature up to a maximum applied field of 1.8 Tesla by VSM magnetometry. The magnetic field was applied both in the plane of the substrate surface and perpendicular to the surface plane, showing that the easy magnetization axis of the FePt film is perpendicular to the film surface (Fig. 5.17) and the easy magnetization of the $\mathrm{Fe}_{3} \mathrm{O}_{4}$ is oriented in the plane of the sample surface.

From the hysteresis loop obtained by applying the magnetic field perpendicular to the film surface, it can be observed that the coercive field of the FePt film $\left(\mathrm{H}_{C}(\mathrm{FePt})=2.3 \mathrm{kOe}\right)$ and the demagnetizing field of the magnetite $\left(\mathrm{H}_{D}\left(\mathrm{Fe}_{3} \mathrm{O}_{4}\right)=4.2 \mathrm{kOe}\right)$ have similar values to those detailed for the $\mathrm{MgO}(001)$ $/ / \mathrm{Fe}_{3} \mathrm{O}_{4} / \mathrm{MgO} / \mathrm{FePt}$ heterostructure. 


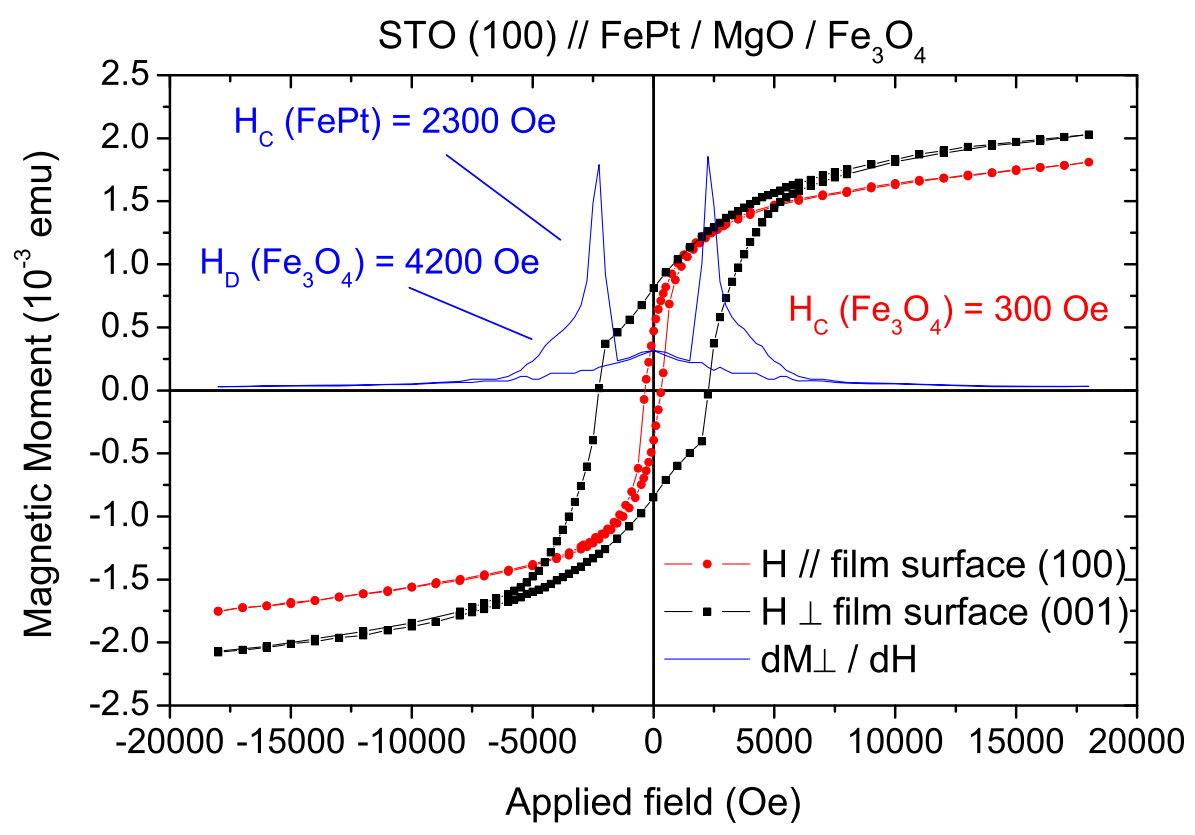

Figure 5.17: RT hysteresis loop of a STO (001) // (16.5 nm) FePt / (3.1 nm) MgO / (33.0 nm) $\mathrm{Fe}_{3} \mathrm{O}_{4}$ heterostructure.

The XRD pattern displayed in figure 5.18 shows the [001] reflections from the $L 1_{0}$ - ordered FePt, which, together with the magnetization measurements, demonstrates the good crystallinity and epitaxial growth of the $L 1_{0}-\mathrm{FePt}$ (001) ordered phase. However, it is observed that the $\mathrm{Fe}_{3} \mathrm{O}_{4}(004)$ does not grow epitaxially, presenting diffraction reflections from different plane families: (111) and (001).

Although the magnetic properties are satisfactories for our objective, from the x-ray characterization, it is evidenced that the crystalline quality of the $\mathrm{MgO}$ barrier and the upper magnetite electrode need to be improved if we want to obtain MTJs from the STO (001) // FePt / $\mathrm{MgO} / \mathrm{Fe}_{3} \mathrm{O}_{4}$ heterostructure. 


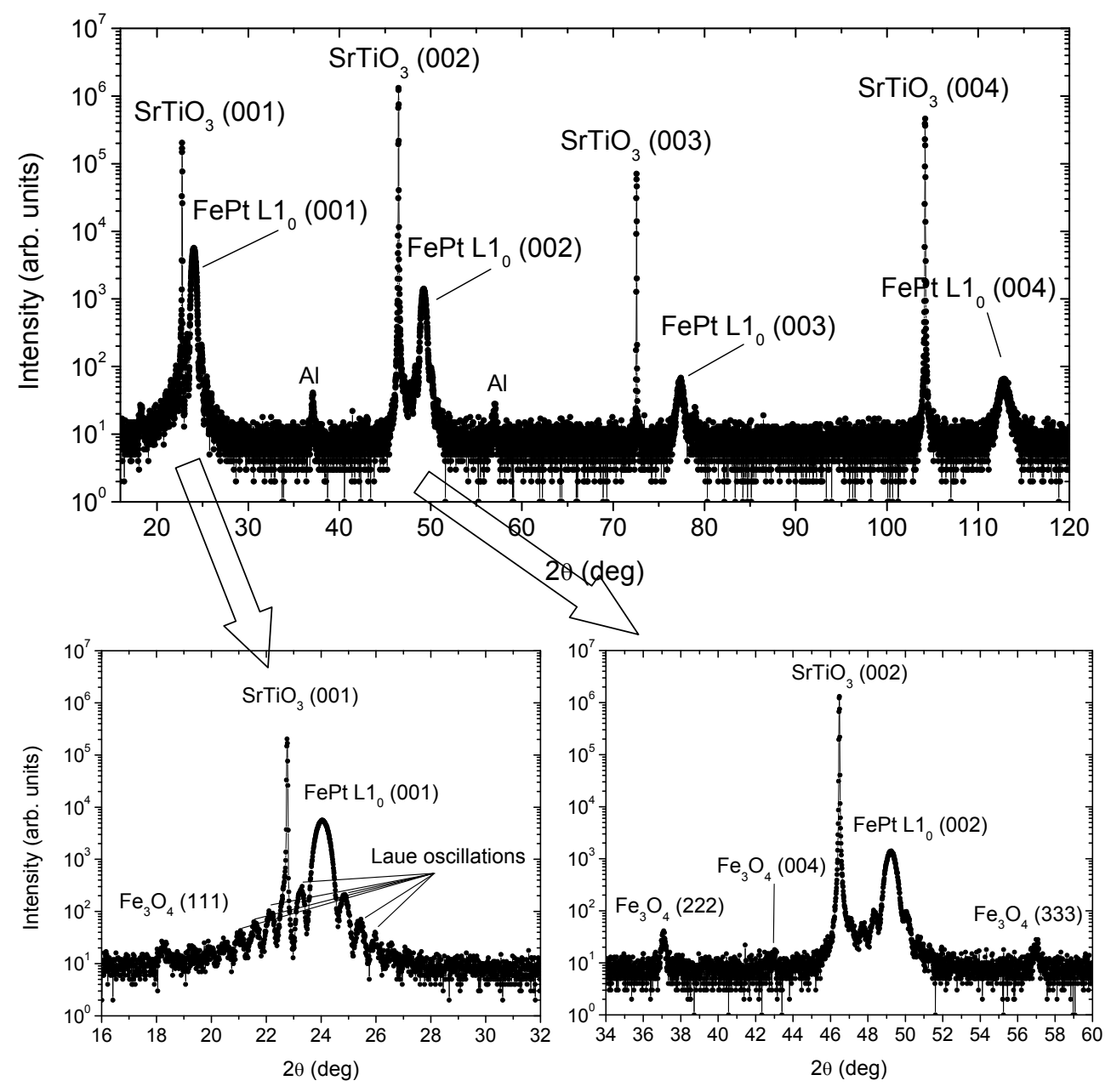

Figure 5.18: $\theta-2 \theta$ scan from a STO (001) // (16.5 nm) FePt / (3.1 nm) MgO / (33.0 $\mathrm{nm}) \mathrm{Fe}_{3} \mathrm{O}_{4}$ heterostructure. 


\subsection{MTJs: Preliminary results}

$\mathrm{CoFeB}_{10}$ is a ferromagnetic material with suitable properties for its inclusion in MTJs, whose growth and magnetotransport properties have been completely optimized at the INESC [274, 275].

Therefore, a $\mathrm{MgO}(001) / /(58.6 \mathrm{~nm}) \mathrm{Fe}_{3} \mathrm{O}_{4} /(1.7 \mathrm{~nm}) \mathrm{MgO} /(3 \mathrm{~nm})$ $\mathrm{CoFeB}_{10} /(5 \mathrm{~nm}) \mathrm{Ru} /(5 \mathrm{~nm})$ Ta heterostructure was grown in order to obtain MTJs by means of optical lithography. The magnetite bottom electrode was deposited by PLD on a $\mathrm{MgO}$ (001) substrate at the INA (Spain), by using standard optimized conditions (See chapter 2).

The $(1.7 \mathrm{~nm}) \mathrm{MgO} /(3 \mathrm{~nm}) \mathrm{CoFeB}_{10} /(5 \mathrm{~nm}) \mathrm{Ru} /(5 \mathrm{~nm})$ Ta structure was grown over the magnetite layer by sputtering using a Nordiko 2000 sputtering system [275] available at the INESC-MN in Lisbon (Portugal). Prior to the sputtering deposition, the sample was heated up to $130{ }^{\circ} \mathrm{C}$ in vacuum for 30 minutes in order to degas its surface.

The Nordiko 2000 is a fully automated magnetron sputtering system with six 3 inch diameter water-cooled magnetrons. All six magnetrons work in DC mode and three of them can also work in RF mode. The system has a small load-lock for loading and unloading the samples into and from the chamber, which maintains a base pressure of $6.6 \times 10^{-8}$ Torr. The growth parameters used for each material are shown in table 5.2 .

\begin{tabular}{||c||c|c||c|c||c||c||c||}
\hline \hline Material & $\begin{array}{c}\mathrm{RF} \\
\text { Power }\end{array}$ & $\begin{array}{c}\mathrm{RF} \\
\text { Bias }\end{array}$ & $\begin{array}{c}\mathrm{DC} \\
\text { Current }\end{array}$ & $\begin{array}{c}\text { DC } \\
\text { Bias }\end{array}$ & $\begin{array}{c}\text { Flow } \\
(\mathrm{sccm})\end{array}$ & $\begin{array}{c}\text { Pressure } \\
(\mathrm{mTorr})\end{array}$ & $\begin{array}{c}\text { Rate } \\
(\AA / \mathrm{s})\end{array}$ \\
\hline \hline $\mathrm{MgO}$ & $130 \mathrm{~W}$ & $296 \mathrm{~V}$ & - & - & 9 & 18.0 & 0.19 \\
\hline $\mathrm{CoFeB}_{10}$ & - & - & $45 \mathrm{~mA}$ & $414 \mathrm{~V}$ & 9 & 7.2 & 0.58 \\
\hline $\mathrm{Ru}$ & - & - & $45 \mathrm{~mA}$ & $294 \mathrm{~V}$ & 8 & 5.2 & 0.40 \\
\hline $\mathrm{Ta}$ & - & - & $45 \mathrm{~mA}$ & $334 \mathrm{~V}$ & 5 & 4.7 & 0.52 \\
\hline \hline
\end{tabular}

Table 5.2: Sputtering deposition conditions.

Once the full heterostructure is deposited, the microfabrication of the MTJs can be started. 


\subsubsection{Magnetic Tunnel Junction Fabrication Process}

The optical lithography process was performed at the $250 \mathrm{~m}^{2}$ clean-room (class 100 and class 10 areas) at the INESC-MN, by means of the following standard microfabrication steps:

\section{Microfabrication Step 1: Bottom electrode definition}

The first step in the microfabrication process consists in defining the bottom electrode shape by etching:

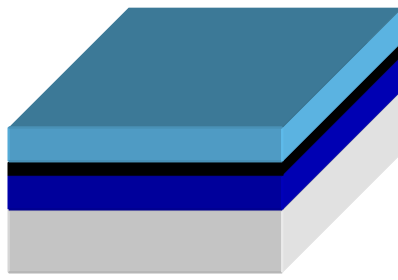

Heterostructure

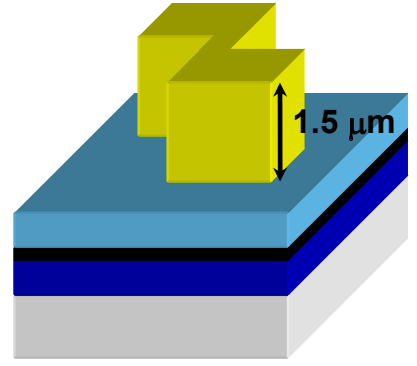

Photoresist deposition

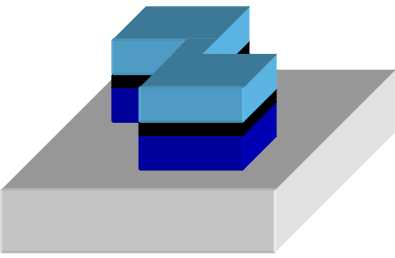

After ion milling and resist strip

Figure 5.19: Microfabrication Step 1: Bottom electrode definition.

The stack deposited on the substrate was coated with a $1.5 \mu \mathrm{m}$ photosensitive polymer (PFR7790G27cP, by JSR Electronics) in a Silicon Valley Group (SVG) system. Then, the pattern was defined by using a DWL 2.0 system from Heidelberg, with a $442 \mathrm{~nm}$ HeCd laser, a laser spot size of $750 \mathrm{~nm}$ and an alignment accuracy of $70 \mathrm{~nm}$. Due to the laser exposure, the chemical bonds of the polymer are broken, and the exposed photoresist can be removed by developing the sample in the SVG system.

Afterwards, the unprotected material from the sample is removed by etching with the Nordiko 3600 ion beam milling automated system. In this system, the substrate table can rotate in plane around its symmetry axis with a rotation speed up to $30 \mathrm{rpm}$, and the relative position between the substrate and the beam can be changed from $0^{\circ}$ to $80^{\circ}$. This works with $\mathrm{Ar}$ and Xe inert gases, and the chamber working pressure is $10^{-8}$ Torr, obtaining an average etch rate for metals of around $1 \AA / \mathrm{s}$. The parameters used for the ion milling are shown in table 5.3

Once the etching process is finished, the remaining photoresist in the sample is removed in a resist strip process, where the sample is heated up to $90{ }^{\circ} \mathrm{C}$ in a solvent called Microstrip and periodically subjected to ultra-sounds. 


\begin{tabular}{||c|c|c|c|c|c||}
\hline \hline $\mathrm{RF}$ & $V^{+}$ & $V^{-}$ & $\mathrm{I}$ & Ar Flow & Pressure \\
\hline $160 \mathrm{~W}$ & $735 \mathrm{~V}$ & $350 \mathrm{~V}$ & $105 \mathrm{~mA}$ & $10 \mathrm{sccm}$ & $1.4 \times 10^{-4}$ Torr \\
\hline \hline
\end{tabular}

Table 5.3: Nordiko 3600 ion milling conditions for the magnetic tunnel junctions full stack.

At the end of the first step, the quality of the process is checked by inspection with an optical microscope (Figure 5.20).

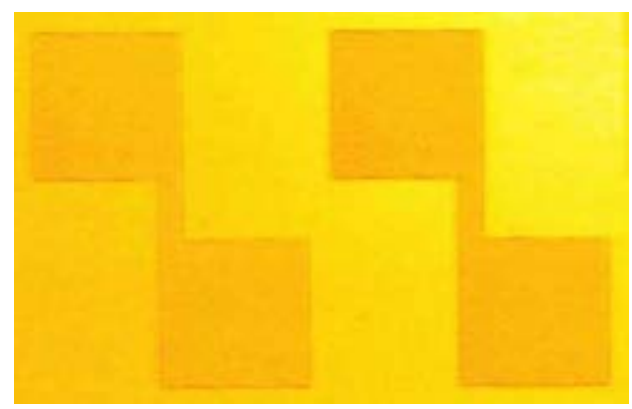

Figure 5.20: Optical microscope image of the pattern obtained after developing the photoresist. The bottom electrode is defined.

\section{Microfabrication Step 2: Definition of the junction area}

The second step in the microfabrication process is also done by etching, in order to define the junction area.

The photoresist deposited with the SVG system is patterned by using Nordiko 2000, defining the junction area as a squared pillar and also covering the two bottom contacts.

After the patterning, the unprotected material is etched by ion milling. The etching must stop in the middle of the structure, right after the tunnel barrier and leaving the bottom electrode almost intact (see figure 5.21).

The second etch is the most critical step of the microfabrication process: if the etch is stopped before reaching the barrier, the junction area will be the whole bottom electrode area and the electrons will flow through pin-holes; on the other hand, if the etch is stopped after etching the lower layers underneath the barrier, there won't be electrical contact to the bottom electrode. In order to improve the control of the etching time, a calibration sample is grown with exactly the same layers and thickness of the sample and used to control the end of the ion milling. 


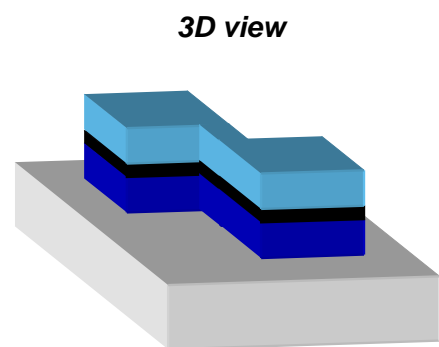

Starting heterostructure

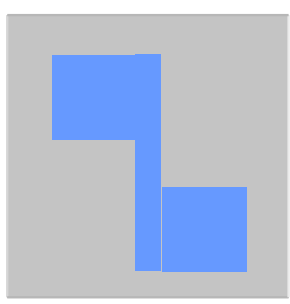

Top view

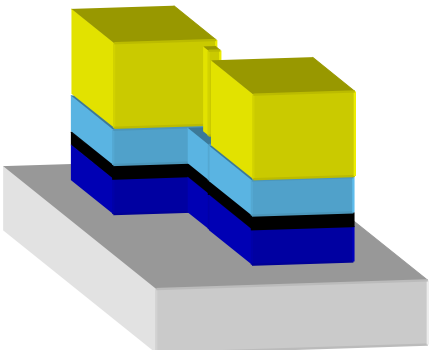

Photoresist deposition

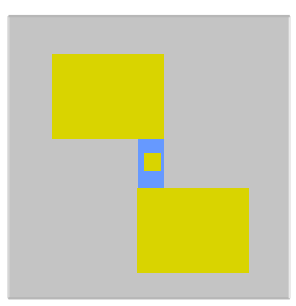

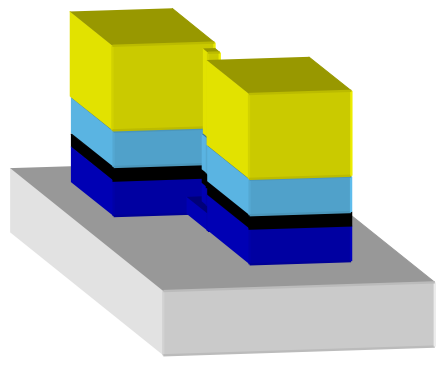

Controlled ion milling

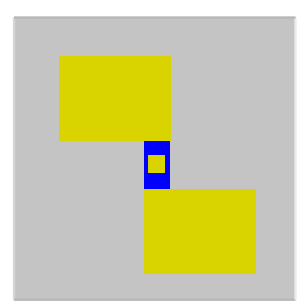

Figure 5.21: Microfabrication Step 2: Definition of the junction area.

The etching conditions have to be carefully controlled in order to avoid material redeposition in the junction sidewalls.

The photoresist must not be removed at the end of this step, as it is necessary for the next step. An image of the sample obtained by an optical microscope after the performance of the second microlithography step can be observed in figure 5.22 .

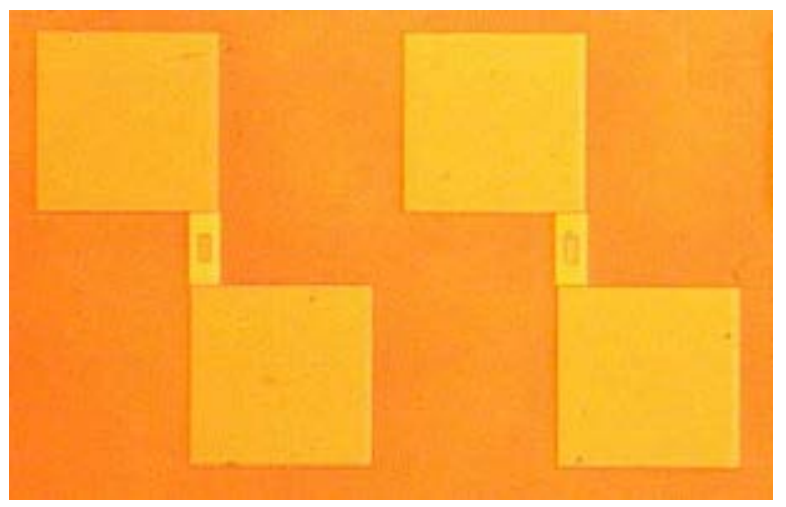

Figure 5.22: Optical microscope image of the pattern obtained after developing the photoresist. The junction area is defined. 


\section{Microfabrication Step 3: Electrode insulation}

A thick insulating layer (tipically $40 \mathrm{~nm}$ of $\mathrm{Al}_{2} \mathrm{O}_{3}$ ) is deposited with an UHVII $\mathrm{RF}$ - magnetron sputtering system in order to avoid any electrical current path between the bottom and top electrodes through anywhere else but the junction area. A base pressure of $\sim 3 \times 10^{-7}$ Torr is required for the optimum deposition conditions described in table 5.4

\begin{tabular}{||c|c|c|c|c||}
\hline \hline Material & RF Power & Gas Flow & Pressure & Deposition Rate \\
\hline $\mathrm{Al}_{2} \mathrm{O}_{3}$ & $200 \mathrm{~W}$ & $45 \mathrm{sccm} \mathrm{Ar}$ & $3.0 \mathrm{mTorr}$ & $0.20 \AA / \mathrm{s}$ \\
\hline \hline
\end{tabular}

Table 5.4: Sputtering parameters in the UHV II system.

In the previous process step the photoresist was not removed, and now it will be used to define the via through the insulation oxide by lift-off (selfaligned process), as shown in Figure 5.23.

The insulator lift-off process duration increases with pattern areas decrease and increasing insulation thickness. Therefore, for junctions with few $\mu m^{2}$ and $40 \mathrm{~nm}$ thick insulating layer, the lift-off can take several days.

\section{Top view}

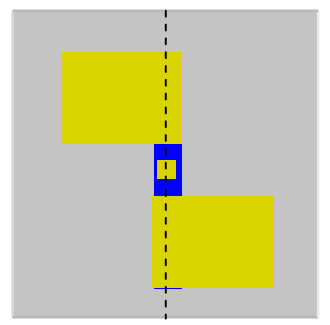

Starting heterostructure

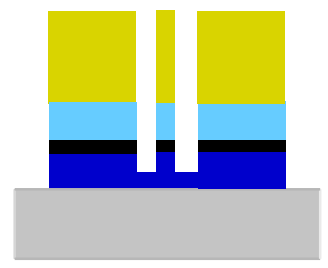

Cut view

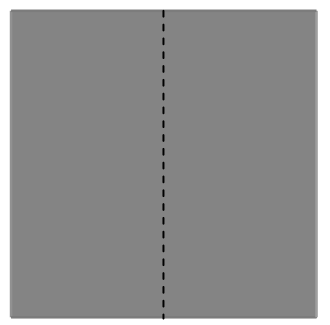

Insulating layer deposition

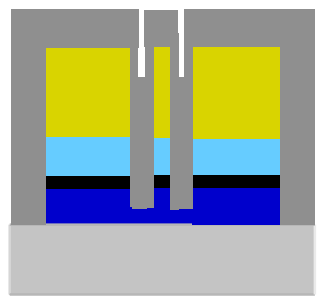

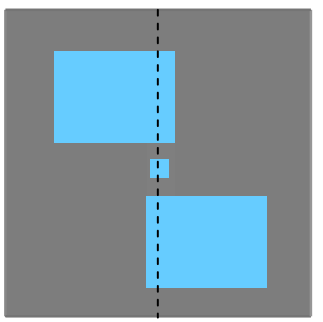

Lift-off

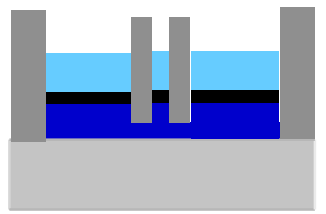

Figure 5.23: Microfabrication Step 3: Bottom electrode insulation. 


\section{Microfabrication Step 4: Top Electrode Contact}

In this process step, both the top and the bottom pads are defined by lift-off as shown in figure 5.24

Top view

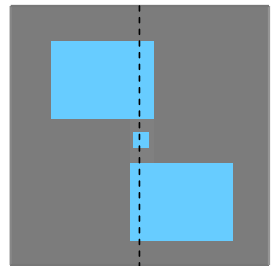

Starting heterostructure

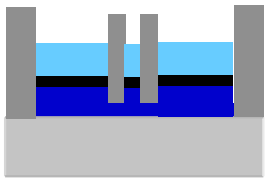

Cut view

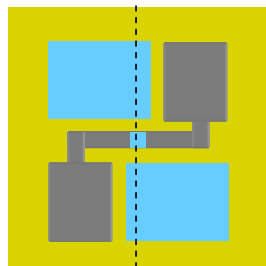

Photoresist deposition and developement

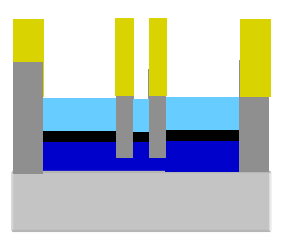

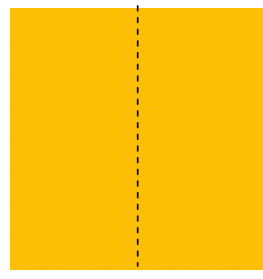

Metal deposition

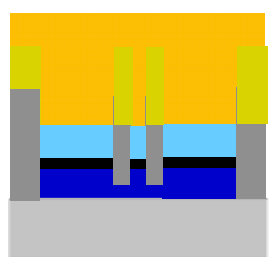

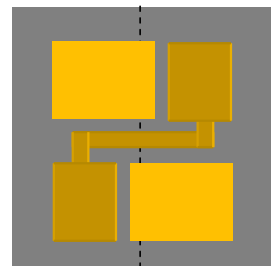

Lift-off

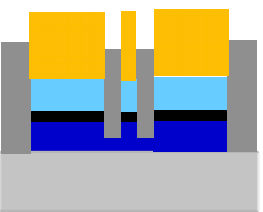

Figure 5.24: Microfabrication Step 4: Top Electrode Contact.

Photoresist is deposited on the sample and is developed in order to define the pattern (Figure 5.25). Afterwards, the sample is covered with metal through a sputter-etch step and two metal layers deposition in the Nordiko 7000 system. After the lift-off, the metal remains only on the contact pads and the top lead.

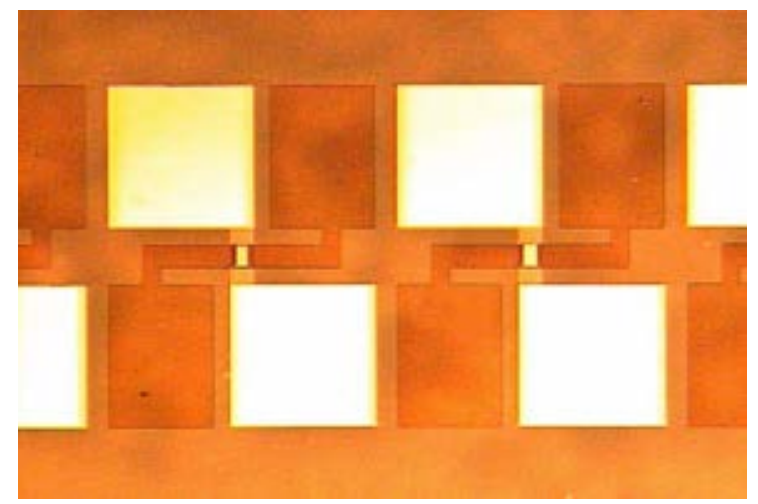

Figure 5.25: Optical microscope picture of the pattern obtained after developing the photoresist. 
The quality of the final lift-off is analysed by optical inspection with a microscope (Figure 5.26) and the magnetic tunnel junction is ready to be measured.

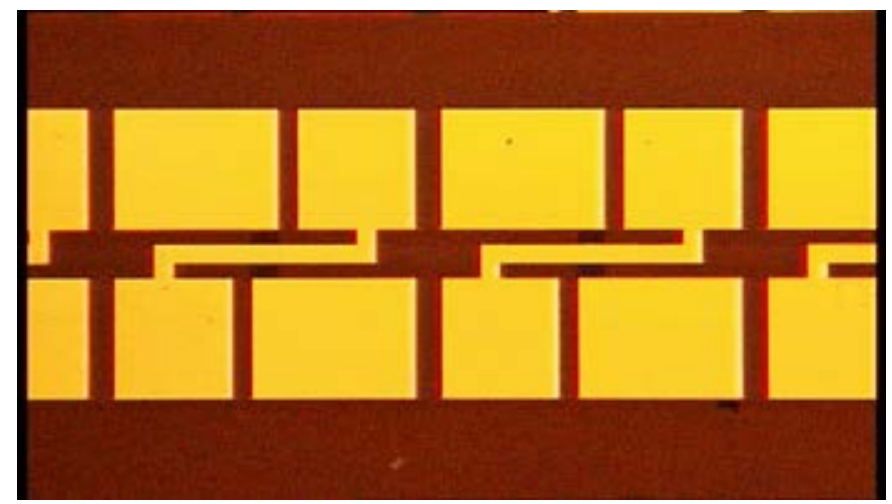

Figure 5.26: Optical microscope picture of processed magnetic tunnel junctions. 


\subsubsection{TMR preliminary measurements}

The tunnel magnetoresistance was measured at the INESC-MN with a manual measurement setup shown in figure 5.27, which consisted on four TiW probes mounted in individual micropositioners with a spatial resolution of $10 \mu \mathrm{m}$, connected to a current source and a voltmeter. The magnetic field was provided by two Helmholtz coils feeded by a second current source.

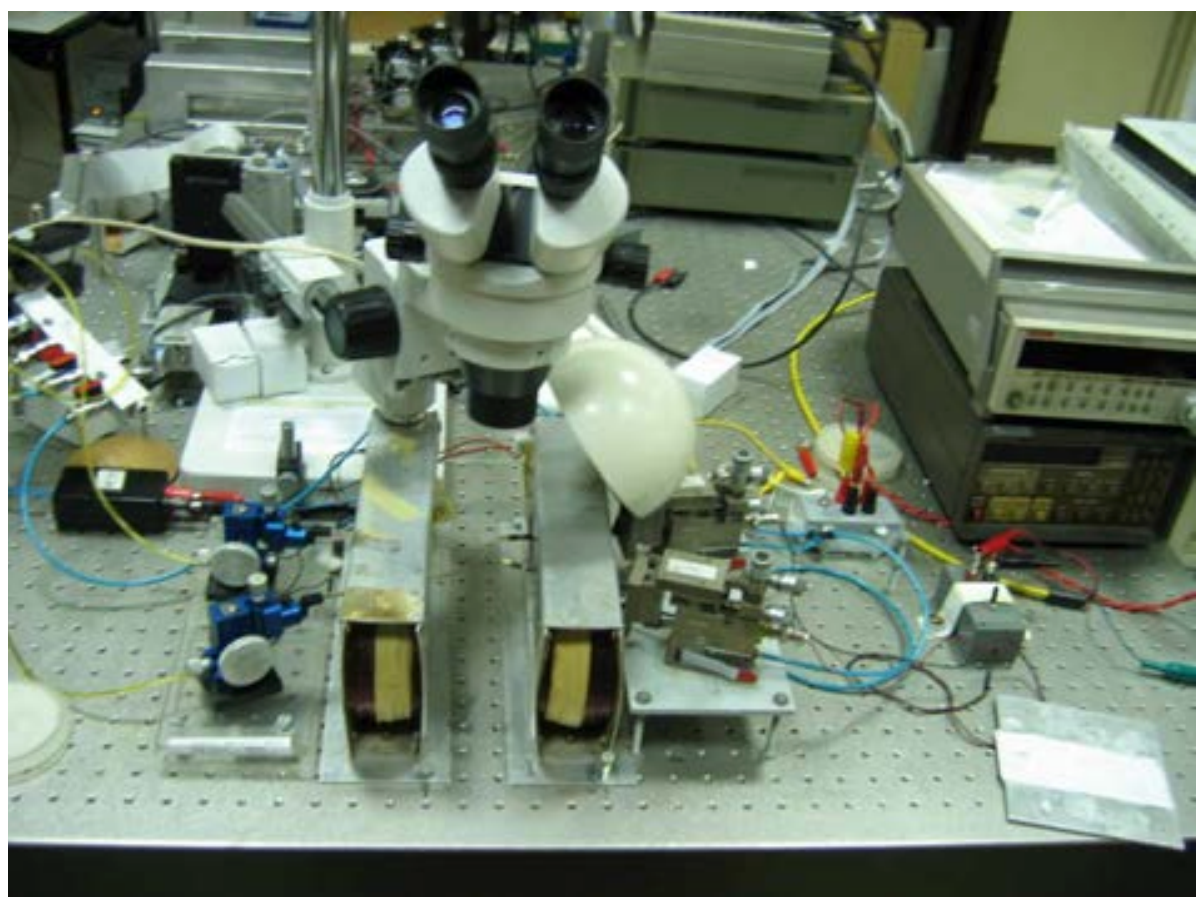

Figure 5.27: Image of the magnetotransport manual measurement setup available at the INESC-MN.

TMR measurements were performed at room temperature up to a maximum in plane magnetic field of 70 Oe on the as-deposited junctions and after performing an annealing of $280{ }^{\circ} \mathrm{C}$ in vacuum, which produces a crystallization of the $\mathrm{CoFeB}_{10}$. At this magnetic field, only the $\mathrm{CoFeB}_{10}$ layer is able to rotate, due to its low coercive field ( 5 - 10 Oe), thus we are measuring a minor loop, and the TMR will be higher than the measured value. The highest TMR ratio was obtained for the bigger as-deposited junction (see figure 5.28). Figure 5.29 shows the TMR ratio of a $3 \mu \mathrm{m} \times 6 \mu \mathrm{m}$ size MTJ, both as deposited and after the annealing.

The TMR results obtained are promising, obtaining ratios up to $8 \%$ which could be increased by improving the heterostructures quality. Therefore, further studies need to be performed, including full-loop TMR measurements. 


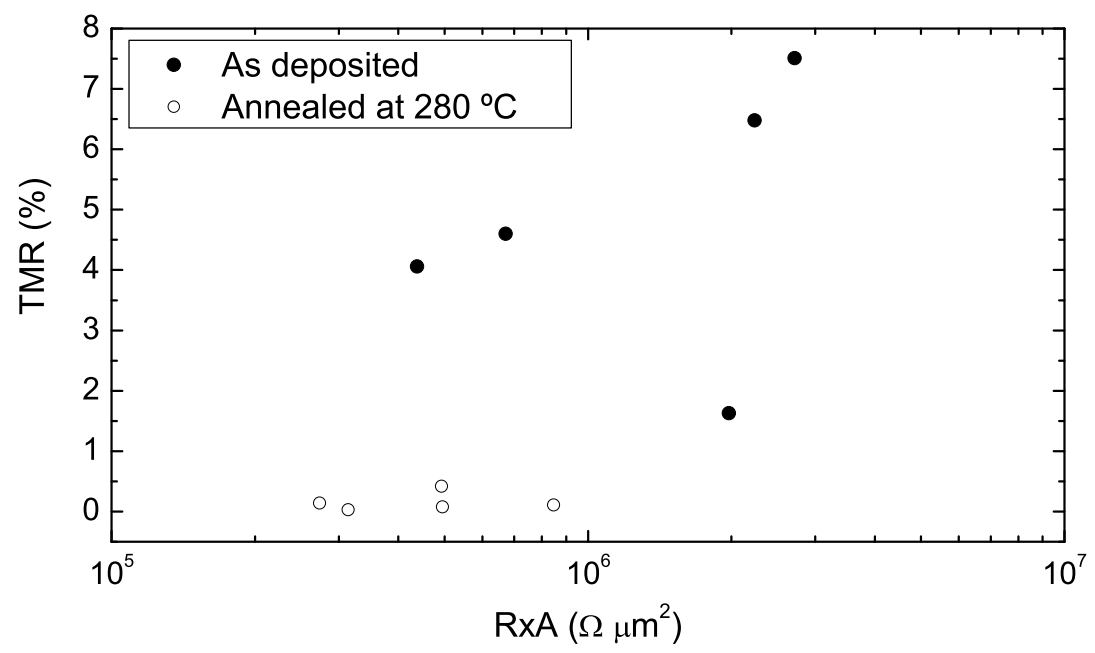

Figure 5.28: TMR ratio of MTJs with the structure $\mathrm{MgO}(001) / /(58.6 \mathrm{~nm}) \mathrm{Fe}_{3} \mathrm{O}_{4}$ / (1.7 nm) $\mathrm{MgO} /(3 \mathrm{~nm}) \mathrm{CoFeB}_{10} /(5 \mathrm{~nm}) \mathrm{Ru} /(5 \mathrm{~nm}) \mathrm{Ta}$ at room temperature, as a function of the Resistance $\times$ Area $(\mathrm{RA})$ product value.

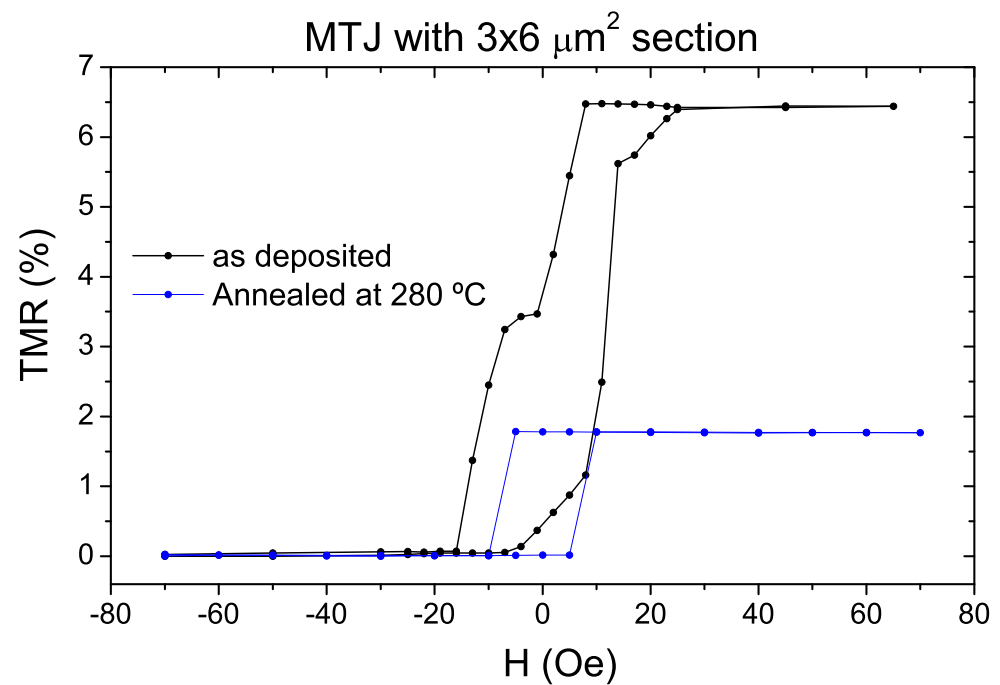

Figure 5.29: TMR ratio of a $3 \mu \mathrm{m} \times 6 \mu \mathrm{m}$ size MTJ with the structure $\mathrm{MgO}(001)$ $/ /(58.6 \mathrm{~nm}) \mathrm{Fe}_{3} \mathrm{O}_{4} /(1.7 \mathrm{~nm}) \mathrm{MgO} /(3 \mathrm{~nm}) \mathrm{CoFeB}_{10} /(5 \mathrm{~nm}) \mathrm{Ru} /(5 \mathrm{~nm}) \mathrm{Ta}$ at room temperature and as a function of the applied magnetic field. 


\subsubsection{MTJs from $\mathrm{Fe}_{3} \mathrm{O}_{4} / \mathrm{MgO} / \mathrm{Fe}$ heterostructures}

Optical lithography is being optimized at the INA (Spain) in order to obtain high-quality MTJs from the $\mathrm{Fe}_{3} \mathrm{O}_{4} / \mathrm{MgO} / \mathrm{Fe}$ heterostructures. Once the multilayer is deposited, the main photolithography steps, up to date, are the followings:

Bottom electrode definition: Deposition of positive photoresist, optical exposition and ion milling of the sample.

Definition of the junction area: The ion milling time have to be thoroughly controlled in order to avoid the bottom electrode destruction.

Electrode insulation: In this case, we use dielectric $\mathrm{Si}_{3} \mathrm{Ni}_{4}$, deposited by plasma-enhanced CVD, up to a thickness of, at least, $100 \mathrm{~nm}$. A reactiveion etching process is performed in order to define the via through the insulator.

Top electrode contact: The top electrode is defined by using invertible photoresist. The metal is deposited by means of e-beam evaporation $(10 \mathrm{~nm}$ $\mathrm{Cr}+150 \mathrm{~nm} \mathrm{Au}$ ), and finally, a lift-off is performed with acetone.

While the MTJs produced at the INESC in Lisbon have a square section, the junctions fabricated at the INA in Zaragoza have circular sections, as can be seen in figure 5.30. These MTJs are being measured at the moment, and results are expected in the next months. 


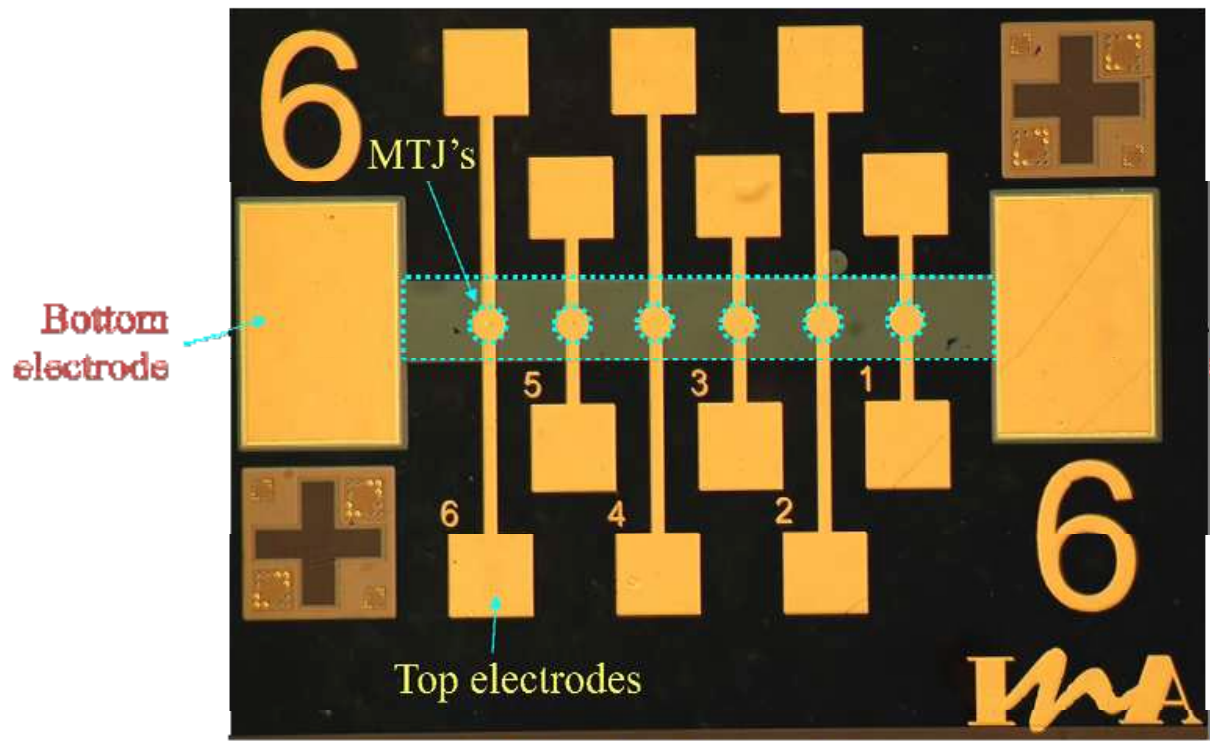

Figure 5.30: Optic microscope image of the MTJs produced at the INA (Spain). 


\section{Conclusions}

This thesis has been devoted to the preparation and characterization of epitaxial thin films and heterostructures which present relevant phenomena at the nanoscale, being specially focused on those systems formed by magnetic oxides produced by pulsed laser ablation, with technological applications in magnetoelectronics. As a result of this work, the following conclusions have been drawn:

- We have grown high-quality epitaxial $\mathrm{Fe}_{3} \mathrm{O}_{4}$ films on $\mathrm{MgO}$ substrates by PLD, performing a thorough structural, morphological, magnetic and electrical transport characterization. The films presented flat and smooth surface with $\mathrm{rms}$ roughness of about $0.1 \mathrm{~nm}$. Typical saturation magnetization values are of the order of $450 \mathrm{emu} / \mathrm{cm}^{3}$ and the Verwey transition was observed in films with different thicknesses, down to $5 \mathrm{~nm}$-thick.

- We have investigated the enhanced magnetic moment found in ultrathin magnetite films ( $<20 \mathrm{~nm}$ ). Contradictory results were obtained when comparing SQUID and VSM magnetometry with PNR and XCMD measurements, e.g., for a $4.5 \mathrm{~nm}$-thick film, a saturation magnetization of $1050 \mathrm{emu} / \mathrm{cm}^{3}$ was found by VSM magnetometry, while the value obtained by PNR was $163 \mathrm{emu} / \mathrm{cm}^{3}$. XPS experiments showed absence of metallic Fe impurities in the magnetite films. Finally, ICP and SQUID magnetometry measurements showed the presence of Fe impurities in the substrates, which indicates that the origin of the enhanced magnetic moment is extrinsic, due to the presence of Fe impurities in the $\mathrm{MgO}$ substrates, and not intrinsic to $\mathrm{Fe}_{3} \mathrm{O}_{4}$.

- We have grown epitaxial $\mathrm{Sr}_{2} \mathrm{CrReO}_{6}$ double-perovskite thin films by PLD with high crystallinity and relatively large cationic order. Typical values of saturation magnetization of $\mathrm{M}_{S}=1 \mu_{B} /$ f.u. and electrical resistivity of $\rho(300 \mathrm{~K})=2.8 \mathrm{~m} \Omega \cdot \mathrm{cm}$ were obtained, in good agreement with previous published results in sputtered epitaxial thin films. We estimate that the AS concentration in our thin films is of the order of $14 \%$, and a direct 
visualization with atomic resolution in real space is being performed at the moment by means of Z-contrast aberration corrected ADF-STEM.

- We have performed a study on the growth of 15 nm-thick $L 1_{0}-F e P t$ films on different substrates and buffer layers, finding the optimal deposition conditions for $\mathrm{MgO}$ (001) and STO substrates, ie, a substrate temperature of $450{ }^{\circ} \mathrm{C}$ for both of them, being necessary an annealing in vacuum for 60 minutes for the growth over STO substrates. These results have allowed us the integration of FePt films on heterostructures with $\mathrm{MgO}$ tunnel barrier and a magnetite electrode for future MTJs including both in-plane and out-of-plane anisotropy geometries in the MTJs electrodes.

- We have produced high quality epitaxial $\mathrm{Fe}_{3} \mathrm{O}_{4} / \mathrm{MgO} / \mathrm{Fe}$ heterostructures, all layers being deposited by PLD. All interfaces appear very sharp with relatively small $\mathrm{rms}$ roughness, $\sim 0.2 \mathrm{~nm}$. The magnetic coupling between $\mathrm{Fe}_{3} \mathrm{O}_{4}$ and $\mathrm{Fe}$ electrodes is also very small, $J \sim 0.03 \mathrm{~mJ} / \mathrm{m}^{2}$ ( $\mathrm{MgO}$ barrier thickness of $2 \mathrm{~nm}$ ). The obtention of Magnetic Tunnel Junctions from these heterostructures by means of optical lithography is in progress. 


\section{Bibliography}

[1] G. Binash, P. Grunberg, F. Saurenbach, and W. Zinn, Phys. Rev. B 39, 4828 (1989).

[2] M. N. Baibich, and J. M. Broto, and A. Fert, and F. Nguyen Van Dau, and F. Petroff, and P. Etienne, and G. Creuzet, and A. Friederich and J. Chazelas, Phys. Rev. Lett. 61, 2472 (1988).

[3] J. M. D. Coey and S. Sanvito, J. Phys. D.: Appl. Phys 37, 988 (2004).

[4] M. Ziese, Reports on Progress in Physics 65, 143 (2002).

[5] R. Lyonnet, A. N. Khodan, A. Barthélémy, J. P. Contour, O. Durand, J. L. Maurice, D. Michel, and J. D. Teresa, J. Electroceramics 4, 369 (2000).

[6] E. S. Vlakhov, R. A. Chakalov, R. I. Chakalova, K. A. Nenkov, K. Dorr, A. Handstein, and K. H. Muller, J. Appl. Phys. 83, 2152 (1998).

[7] V. A. Vasko, C. A. Nordman, P. A. Kraus, V. S. Achutharaman, A. R. Ruosi, and A. M. Goldman, Appl. Phys. Lett. 68, 2571 (1996).

[8] V. Moshnyaga, I. Khoroshun, A. Sidorenko, P. Petrenko, A. Weidinger, M. Zeitler, B. Rauschenbach, R. Tidecks, and K. Samwer, Appl. Phys. Lett. 74, 2842 (1999).

[9] S. K. Arora, H.-C. Wu, H. Yao, W. Y. Ching, R. J. Choudhary, I. V. Shvets, and O. N. Mryasov, IEEE Trans. Magn. 44, 2628 (2008).

[10] S. K. Arora, H.-C. Wu, R. J. Choudhary, I. V. Shvets, O. N. Mryasov, H. Yao, and W. Y. Ching, Phys. Rev. B 77, 134443 (2008).

[11] J. S. Moodera, L. R. Kinder, T. M. Wong, and R. Meservey, Phys. Rev. Lett. 74, 3273 (1995).

[12] T. Miyazaki and N. Tezuka, J. Magn. Magn. Mater. 139, 231 (1995).

[13] R. P. Feynman, Engineering and Science 23, 22 (1960). 
[14] N. Taniguchi, Proc. Intl. Conf. Prod. Eng. Tokyo, Part II (1974).

[15] M. F. Ashby, P. J. Ferreira, and D. L. Schodek, Nanomaterials, Nanotechnologies and Design: An Introduction for Engineers and Architects (Elsevier, 2009).

[16] L. Royer, Bull. Soc. Franc. Mineral 51, 7 (1928).

[17] L. B. Freund, Scripta Met. Mater 27, 669 (1992).

[18] M. Ohring, Materials science of thin films (Academic Press, 2001), 2nd ed.

[19] E. Bauer, Int. Zeit. Kristallogr. 110, 372 (1958).

[20] Pulsed Laser Deposition of Thin Films (Douglas B. Chrisey and Graham K. Hubler, 1994), john wiley \& sons ed.

[21] I. N. Stranski and L. V. Krastanow, Akad. Wiss. Lit. Mainz Abh. Math. Naturwiss. Kl. 146, 797 (1939).

[22] V. E. Henrich, G. Dresselhaus, and H. J. Zeiger, Phys. Rev. B 17, 4908 (1978).

[23] M. Kawasaki, K. Takahashi, T. Maeda, R. Tsuchiya, M. Shinohara, O. Ishiyama, T. Yonezawa, M. Yoshimoto, and H. Koinuma, Science 266, 1540 (1994).

[24] M. Kawasaki, A. Ohtomo, T. Arakane, K. Takahashi, M. Yoshimoto, and H. Koinuma, Appl. Surf. Sci. 107, 102 (1996).

[25] Y. Liang and D. A. Bonnell, Surf. Sci. Lett. 285, L510 (1993).

[26] T. Nishimura, A. Ikeda, H. Namba, T. Morishita, and Y. Kido, Surf. Sci. 421, 273 (1999).

[27] T. Matsumoto, H. Tanaka, T. Kawai, and S. Kawai, Surf. Sci. Lett. 278, L153 (1992).

[28] Q. D. Jiang and J. Zegenhagen, Surf. Sci. 338, L882 (1995).

[29] URL wWw.pcguide.com/ref/hdd/.

[30] G. E. Moore, Electronics 38, 114 (1965).

[31] A. Pohm, J. Daughton, C. Comstock, H. Yoo, and J. Hur, IEEE Trans. Magn. 23, 2575 (1987). 
[32] A. V. Pohm, J. S. T. Huang, J. M. Daughton, D. R. Krahn, and V. Mehra, IEEE Trans. Magn. 24, 3117 (1988).

[33] S. A. Wolf, A. Y. Chtchelkanova, and D. M. Treger, IBM J. Res. and Dev. 50, 101 (2006).

[34] S. A. Wolf, D. D. Awschalom, R. A. Buhrmann, J. M. Daughton, S. von Molnar, M. L. Roukes, A. Y. Chtchelkanova, and D. M. Treger, Science 294, 1488 (2001).

[35] E. Grochowski and R. E. Fontana, Nanofilms in Giant Magnetoresistance Heads (Dekker Encyclopedia of Nanoscience and Nanotechnology, 2004).

[36] G. A. Prinz, J. Magn. Magn. Mater. 200, 57 (1999).

[37] I. Zutic, J. Fabian, and S. D. Sarma, Rev. Mod. Phys. 76, 323 (2004).

[38] M. Tanaka, J. Crystal Growth 278, 25 (2005).

[39] A. Fert, Thin Solid Films 517, 2 (2008).

[40] N. H. Mott, Proc. Roy. Soc. London Ser. A 153, 699 (1936).

[41] URL wWw.spectrosciences.com/spip.php?breve426

[42] B. Dieny, V. S. Speriosu, S. S. P. Parkin, B. A. Gurney, D. R. Wilhoit, and D. Mauri, Phys. Rev. B 43, 1297 (1991).

[43] M. Jullière, Phys. Lett. A 54, 225 (1975).

[44] A. Schuhl and D. Lacour, C. R. Physique 6, 945 (2005).

[45] S. Yuasa, AIST Today 5, 25 (2005).

[46] S. Yuasa, T. Nagahama, A. Fukushima, Y. Suzuki, and K. Ando, Nat. Mater. 3, 868 (2004).

[47] URL www.nims.go.jp/apfim/halfmetal.html.

[48] R. A. de Groot, F. M. Mueller, P. G. van Engen, and K. H. J. Buschow, Phys. Rev. Lett. 50, 2024 (1983).

[49] URL www.riken.go.jp/lab-www/nanomag/research/half_metals_e.html

[50] S. Parkin, C. Kaiser, A. Panchula, P. Rice, B. Hughes, M. Samant, and S.-H. Yang, Nat. Mater. 3, 862 (2004).

[51] D. D. Djayaprawira, K. Tsunekawa, M. Nagai, H. Maehara, S. Yamagata, N. Watanabe, S. Yuasa, Y. Suzuki, and K. Ando, Appl. Phys. Lett. 86, 092502 (2005). 
[52] J. F. Ready, Appl. Phys. Lett. 3, 11 (1963).

[53] H. Schwartz and A. J. Demaria, Proc. National Electronics Conf. 18, 351 (1962).

[54] H. M. Smith and A. F. Turner, Appl. Opt 4, 147 (1965).

[55] H. Sankur, Appl. Opt 25, 1962 (1986).

[56] D. Dijkkamp, T. Venkatesan, X. D. Wu, S. A. Shaheen, N. Jisrawi, Y. H. Min-Lee, W. L. McLean, and M. Croft, Appl. Phys. Lett. 51, 619 (1987).

[57] H. Schwartz and H. A. Tourtellotte, J. Vac. Sci. Technol. 6, 373 (1969).

[58] R. Kelly, J. J. Cuomo, P. A. Leary, J. E. Rothenberg, B. E. Braren, and C. F. Aliotta, Nucl. Instrum. Methods Phys. Res. B9, 329 (1985).

[59] P. R. Willmott, Prog. Surf. Sci. 76, 163 (2004).

[60] R. J. Lade and M. N. Ashfold, Surf. Coat. Technol. 120-121, 313 (1999).

[61] URL www.chm.bris.ac.uk/laser/ashfold/ablation.htm.

[62] M. G. Norton and C. B. Carter, Physica C 172, 47 (1990).

[63] T. Ohnishi, K. Shibuya, T. Yamamoto, and M. Lippmaa, J. Appl. Phys. 103, 103703 (2008).

[64] S. M. Rossnagel and K. L. Saenger, J. Vac. Sci. Technol. A7, 968 (1989).

[65] D. A. Glocker and S. I. Shah, Handbook of Thin Film Process Technology (Douglas B. Chrisey and Graham K. Hubler, Techno House, Bristol, 1995), iop publishing ed.

[66] F. D. Czeschka, Ph.D. thesis, Munchen University, Facolta di Scienze Matematiche Fisiche Naturali (2007).

[67] F. Huang, University of Alabama (MINT Center, 2005).

[68] G. Binning, C. F. Quate, and C. Gerber, Phys. Rev. Lett. 56, 930 (1985).

[69] URL www.physics.leidenuniv.nl/sections/cm/ip/group/Principle_of_SPM.htm.

[70] P. J. Flanders, J. Appl. Phys. 63, 3940 (1988).

[71] F. Ott, J. Phys.: Condens. Matter 20, 264009 (2008).

[72] M. Björck and G. Andersson, J. Appl. Cryst. 40, 1174 (2007).

[73] R. Cubitt and G. Fragneto, Appl. Phys. A 74, S329 (2002). 
[74] V. der Pauw, Philips technical review 20, 220 (1958).

[75] A. Fernández-Pacheco, Ph.D. thesis, Universidad de Zaragoza, Departamento. Física de la Materia condensada (2009).

[76] P. Weiss, J. de Phys. 5, 435 (1896).

[77] A. Yanase and K. Siratori, J. Phys. Soc. Jpn. 53, 312 (1984).

[78] S. F. Alvarado, W. Eib, F. Meier, D. T. Pierce, K. Sattler, and H. C. Siegmann, Phys. Rev. Lett. 34, 319 (1975).

[79] E. J. W. Verwey, Nature 144, 327 (1939).

[80] E. J. W.Verwey and P. W. Haayman, Physica 8, 979 (1941).

[81] E. J. Verwey, P. W. Haayman, and F. C. Romeijn, J. Chem. Phys. 15, 181 (1947).

[82] Z. Szotek, W. M. Temmerman, A. Svane, L. Petit, P. Strange, G. M. Stocks, D. Ködderitzsch, W. Hergert, and H. Winter, J. Phys.: Condens. Matter 16, S5587 (2004).

[83] Z. Zhang and S. Satpathy, Phys. Rev. B 44, 13319 (1991).

[84] R. A. de Groot and K. H. J. Buschow, J. Magn. Magn. Mater. 54-57, 1377 (1986).

[85] J. Chen, D. J. Huang, A. Tanaka, C. F. Chang, S. C. Chung, W. B. Wu, and C. T. Chen, Phys. Rev. B 69, 085107 (2004).

[86] G. K. Rozenberg, M. P. Pasternak, W. M. Xu, Y. Amiel, M. Hanfland, M. Amboage, R. D. Taylor, and R. Jeanloz, Phys. Rev. Lett. 96, 045705 (2006).

[87] G. Hu and Y. Suzuki, Phys. Rev. Lett. 89, 276601 (2002).

[88] J. Y. T. Wei, N. C. Yeh, R. P. Vasquez, and A. Gupta, J. Appl. Phys. 83, 7366 (1998).

[89] P. Seneor, A. Fert, J.-L. Maurice, F. Montaigne, F. Petroff, and A. Vaurès, Appl. Phys. Lett. 74, 4017 (1999).

[90] F. Walz, J. Phys.: Condens. Matter 14, R285 (2002).

[91] J. García and G. Subías, J. Phys.: Condens. Matter 16, R145 (2004).

[92] A. Muan and E. Osborn, Phase Equilibria Among Oxides in Steelmaking (1965), oxford: addison-wesley ed. 
[93] W. H. Bragg, Nature 95, 561 (1915).

[94] URL www.ruf.rice.edu/\$\sim\$natelson/magnetite_str_lg.png.

[95] L. Nèel, Ann. Phys. Fr 3, 137 (1948).

[96] R. W. Millar, J. Am. Chem. Soc. 51, 215 (1929).

[97] C. H. Li, Phys. Rev. 40, 1002 (1932).

[98] S. B. Ogale, K. Ghosh, R. P. Sharma, R. L. Greene, R. Ramesh, and T. Venkatesan, Phys. Rev. B 57, 7823 (1998).

[99] M. Pai and J. M. Honig, J. Phys. C: Solid State Phys. 16, L35 (1983).

[100] J. R. Drabble, T. D. Whyte, and R. M. Hooper, Solid State Commun. 9, 275 (1971).

[101] J. S. Y. Feng, R. D. Pashley, and M. A. Nicolet, J. Phys. C: Solid State Phys. 8, 1010 (1975).

[102] N. C. Tombs and H. P. Rooksby, Acta. Cryst. 4, 474 (1951).

[103] H. P. Rooksby and B. T. M. Willis, Acta. Cryst. 6, 565 (1953).

[104] S. C. Abrahams and B. A. Calhoun, Acta. Cryst. 8, 257 (1955).

[105] M. Iizumi, T. F. Koetzle, G. Shirane, S. Chikazumi, M. Matsui, and S. Todo, Acta. Cryst. B38, 2121 (1982).

[106] V. A. M. Brabers, F. Walz, and H. Kronmüller, Phys. Rev. B 58, 14163 (1998).

[107] G. A. Samara, Phys. Rev. Lett. 21, 795 (1968).

[108] S. K. Ramasesha, M. Mohan, A. K. Singh, J. M. Honig, and C. N. R. Rao, Phys. Rev. B 50, 13789 (1994).

[109] G. K. Rozenberg, G. R. Hearne, M. P. Pasternak, P. A. Metcalf, and J. M. Honig, Phys. Rev. B 53, 6482 (1996).

[110] G. K. Rozenberg, Y. Amiel, W. M. Xu, M. P. Pasternak, R. Jeanloz, M. Hanfland, and R. D. Taylor, Phys. Rev. B 75, 020102(R) (2007).

[111] R. Aragón, D. J. Buttrey, J. P.Shepherd, and J. M. Honig, Phys. Rev. B 31, 430 (1985).

[112] J. P.Shepherd, R. Aragón, J. W. Koenitzer, and J. M. Honig, Phys. Rev. B 32, 1818 (1985). 
[113] Z. Kąkol and J. M. Honig, Phys. Rev. B 40, 9090 (1989).

[114] J. P. Shepherd, J. W. Koenitzer, R. Aragón, J. Spa ұek, and J. M. Honig, Phys. Rev. B 43, 8461 (1991).

[115] R. Aragón, P. M. Gehring, and S. M. Shapiro, Phys. Rev. Lett. 70, 1635 (1993).

[116] T. Fujii, M. Takano, R. Katano, Y. Bando, and Y. Isozumi, J. Crystal Growth 99, 606 (1990).

[117] Y. S. Dedkov, U. Rüdiger, and G. Güntherodt, Phys. Rev. B 65, 064417 (2002).

[118] E. Lochner, K. A. Shaw, R. C. DiBari, W. Portwine, P. Stoyonov, S. D. Berry, and D. M. Lind, IEEE Trans. Magn. 30, 4912 (1994).

[119] A. V. Ramos, J. B. Moussy, M. J. Guittet, A. M. Bataille, M. GautierSoyer, M. Viret, C. Gatel, P. Bayle-Guillemaud, and E. Snoeck, J. Appl. Phys. 100, 103902 (2006).

[120] S. K. Arora, R. G. S. Sofin, I. V. Shvets, and M. Luysberg, J. Appl. Phys. 100, 073908 (2006).

[121] D. T. Margulies, F. T. Parker, and A. E. Berkowitz, J. Appl. Phys. 75, 6097 (1994).

[122] H. Qiu, L. Pan, L. Li, H. Zhu, X. Zhao, M. Xu, L. Qin, and J. Q. Xiao, J. Appl. Phys. 102, 113913 (2007).

[123] J. F. Bobo, D. Basso, E. Snoeck, C. Gatel, D. Hrabovsky, J. L. Gauffier, L. Ressier, R. Mamy, S. Visnovsky, J. Hamrle, et al., Eur. Phys. J. B. 24, 43 (2001).

[124] X. W. Li, A. Gupta, G. Xiao, and G. Q. Gong, J. Appl. Phys. 83, 7049 (1998).

[125] M. G. Chapline and S. X. Wang, J. Appl. Phys. 97, 123901 (2005).

[126] A. Bollero, M. Ziese, R. Höhne, H. C. Semmelhack, U. Köhler, A. Setzer, and P. Esquinazi, J. Magn. Magn. Mater. 285, 279 (2005).

[127] S. P. Sena, R. A. Lindley, H. J. Blythe, C. Sauer, M. Al-Kafarji, and G. A. Gehring, J. Magn. Magn. Mater. 176, 111 (1997).

[128] G. Q. Gong, A. Gupta, G. Xiao, W. Qian, and V. P. Dravid, Phys. Rev. B 56, 5096 (1997). 
[129] D. T. Margulies, F. T. Parker, F. E. Spada, R. S. Goldman, J. Li, R. Sinclair, and A. E. Berkowitz, Phys. Rev. B 53, 9175 (1996).

[130] D. T. Margulies, F. T. Parker, M. L. Rudee, F. E. Spada, J. N. Chapman, P. R. Aitchison, and A. E. Berkowitz, Phys. Rev. Lett. 79, 5162 (1997).

[131] F. C. Voogt, T. T. M. Palstra, L. Nielsen, O. C. Rogojanu, M. A. James, and T. Hibma, Phys. Rev. B 57, R8107 (1998).

[132] W. Eerenstein, T. Hibma, and S. Celotto, Phys. Rev. B 70, 184404 (2004).

[133] W. Eerenstein, T. T. M. Palstra, T. Hibma, and S. Celotto, Phys. Rev. B 66, 201101(R) (2002).

[134] W. Eerenstein, T. T. M. Palstra, S. S. Saxena, and T. Hibma, Phys. Rev. Lett. 88, 247204 (2002).

[135] M. Ziese and H. J. Blythe, J. Phys.: Condens. Matter 12, 13 (2000).

[136] T. Kado, J. Appl. Phys. 103, 043902 (2008).

[137] W. Eerenstein, T. T. M. Palstra, T. Hibma, and S. Celotto, Phys. Rev. B 68, 014428 (2003).

[138] S. K. Arora, R. G. S. Sofin, A. Nolan, and I. V. Shvets, J. Magn. Magn. Mater. 286, 463 (2005).

[139] Y. Zhou, X. Jin, and I. V. Shvets, J. Magn. Magn. Mater. 286, 346 (2005).

[140] M. Luysberg, R. G. S. Sofin, S. K. Arora, and I. V. Shvets, Phys. Rev. B 80, 024111 (2009).

[141] S. Celotto, W. Eerenstein, and T. Hibma, Eur. Phys. J. B. 36, 271 (2003).

[142] V. S. Speriosu, M. M. Chen, and T. . Suzuki, IEEE Trans. Magn. 25, 3875 (1989).

[143] A. M. Bataille, L. Ponson, S. Gota, L. Barbier, D. Bonamy, M. GautierSoyer, C. Gatel, and E. Snoeck, Phys. Rev. B 74, 155438 (2006).

[144] J. B. Moussy, S. Gota, A. Bataille, M. J. Guittet, M. Gautier-Soyer, F. Delille, B. Dieny, F. Ott, T. D. Doan, P. Warin, et al., Phys. Rev. B 70, 174448 (2004).

[145] P. J. van der Zaag, W. F. J. Fontijn, P. Gaspard, R. M. Wolf, V. A. M. Brabers, R. J. M. van de Veerdonk, and P. A. A. van der Heijden, J. Appl. Phys. 79, 5936 (1996). 
[146] C. Park, Y. Peng, J. G. Zhu, D. E. Laughlin, and R. M. White, J. Appl. Phys. 97, 10C303 (2005).

[147] H. Li, Y. Wu, Z. Guo, S. Wang, K. L. Teo, and T. Veres, Appl. Phys. Lett. 86, 252507 (2005).

[148] D. Serrate, J. M. D. Teresa, P. A. Algarabel, R. Fernández-Pacheco, J. Galibert, and M. R. Ibarra, J. Appl. Phys. 972, 084317 (2005).

[149] D. Reisinger, P. Majewski, M. Opel, L. Alff, and R. Gross, Appl. Phys. Lett. 85, 4980 (2004).

[150] Y. Zhou, C. McEvoy, R. Ramos, and I. V. Shvets, J. Appl. Phys. 99, 08J111 (2006).

[151] A. Fernández-Pacheco, J. M. D. Teresa, J. Orna, L. Morellón, P. A. Algarabel, J. A. Pardo, and M. R. Ibarra, Phys. Rev. B 78, 212402 (2008).

[152] J. M. D. Teresa, A. Fernández-Pacheco, L. Morellón, J. Orna, J. A. Pardo, D. Serrate, P. A. Algarabel, and M. R. Ibarra, Mic. Eng. 84, 1660 (2007).

[153] A. Fernández-Pacheco, J. M. D. Teresa, J. Orna, L. Morellón, P. A. Algarabel, J. A. Pardo, and M. R. Ibarra, Phys. Rev. B 77, 100403(R) (2008).

[154] A. Fernández-Pacheco, J. Orna, J. M. D. Teresa, P. A. Algarabel, L. Morellón, J. A. Pardo, and M. R. Ibarra, Appl. Phys. Lett. 95, 262108 (2009).

[155] I. A. Campbell and A. Fert, Ferromagnetic Materials, vol. 3 (E. P. Wohlfarth, North-Holland, Amsterdam, 1982).

[156] G. Li, T. Yang, Q. Hu, H. Jiang, and W. Lai, Phys. Rev. B 65, 134421 (2002).

[157] S. T. B. Goennenwein, R. S. Keizer, S. W. Schink, I. V. Dijk, T. M. Klapwijk, and G. X. Miao, Appl. Phys. Lett. 90, 142509 (2007).

[158] A. Schuhl, F. N. V. Dau, and J. R. Childress, Appl. Phys. Lett. 66, 2751 (1995).

[159] A. O. Adeyeye, M. T. Win, T. A. Tan, G. S. Chong, V. Ng, and T. S. Low, Sens. Actuators A 116, 95 (2004).

[160] H. X. Tang, R. K. Kawakami, D. D. Awschalom, and M. L. Roukes, Phys. Rev. Lett. 90, 107201 (2003). 
[161] K. Y. Wang, K. W. Edmonds, R. P. Campion, L. X. Zhao, C. T. Foxon, and B. L. Gallagher, Phys. Rev. B 72, 085201 (2005).

[162] W. L. Lim, X. Liu, K. Dziatkowski, Z. Ge, S. Shen, J. K. Furdyna, and M. Dobrowolska, J. Appl. Phys. 99, 08D505 (2006).

[163] D. Y. Shin, S. J. Chung, S. Lee, X. Liu, and J. K. Furdyna, Phys. Rev. Lett. 98, 047201 (2007).

[164] D. Y. Shin, S. J. Chung, S. Lee, X. Liu, and J. K. Furdyna, IEEE Trans. Magn. 43, 3025 (2007).

[165] X. Jin, R. Ramos, Y. Zhou, C. McEvoy, and I. V. Shvets, J. Appl. Phys. 99, 08C509 (2006).

[166] Y. Bason, L. Klein, H. Q. Wang, J. Hoffman, X. Hong, V. E. Henrich, and C. H. Ahn, J. Appl. Phys. 101, 09J507 (2007).

[167] S. Onoda, N. Sugimoto, and N. Nagaosa, Phys. Rev. Lett. 97, 126602 (2006).

[168] J.-Y. Feng, R. D. Pashley, and M.-A. Nicolet, J. Phys. C: Sol. State Phys. 8, 1010 (1975).

[169] J. Lavine, Phys. Rev 123, 1273 (1961).

[170] S. Todo, K. Siratori, and S. Kimura, J. Phys. Soc. Jpn. 64, 2118 (1995).

[171] J. M. Lavine, Phys. Rev 114, 482 (1959).

[172] W. J. Siemons, IBM J. Res. Dev 14, 245 (1970).

[173] U. Lüders, M. Bibes, J. F. Bobo, M. Cantoni, R. Bertacco, and J. Fontcuberta, Phys. Rev. B 71, 134419 (2005).

[174] M. C. Richter, J. M. Mariot, O. Heckmann, L. Kjeldgaard, B. S. Mun, X. S. Fadley, U. Lüders, J. F. Bobo, P. D. Padova, A. Taleb-Ibrahimi, et al., Eur. Phys. J. Special Topics 169, 175 (2009).

[175] J. Orna, P. A. Algarabel, L. Morellon, J. A. Pardo, J. M. D. Teresa, R. López-Antón, F. Bartolomé, L. M. García, J. Bartolomé, J. C. Cezar, et al., Phys. Rev. B 81, 144420 (2010).

[176] L. G. Parratt, Phys. Rev. 95, 359 (1954).

[177] A. R. Ball, A. J. G. Leenaers, P. J. van der Zaag, K. A. Shaw, B. Singer, D. M. Lind, H. Frederikze, and M. T. Rekveldt, Appl. Phys. Lett. 69, 1489 (1996). 
[178] A. R. Ball, H. Frederikze, D. M. Lind, R. M. Wolf, P. J. H. Bloemen, M. T. Rekveldt, and P. J. van der Zaag, Phys. B: Condensed Matter 221, 388 (1996).

[179] T. Saitoh, Phys. Rev. B 52, 7934 (1995).

[180] J. Park, Nat.Mater. 3, 891 (2004).

[181] B. T. Thole, Phys. Rev. Lett. 68, 1943 (1992).

[182] P. Carra, Phys. Rev. Lett. 70, 694 (1993).

[183] N. Pérez, F. Bartolomé, L. M. García, J. Bartolomé, M. P. Morales, C. J. Serna, A. Labarta, and X. Batlle, Appl. Phys. Lett. 94, 093108 (2009).

[184] T. Fujii, F. M. F. de Groot, G. A. Sawatzky, F. C. Voogt, T. Hibma, and K. Okada, Phys. Rev. B 59, 3195 (1999).

[185] S. A. Krasnikov, A. S. Vinogradov, K. H. Hallmeier, R. Höhne, M. Ziese, P. Esquinazi, T. Chassé, and R. Szargan, Mat. Sci. Eng. B 109, 207 (2004).

[186] M. A. Garcia, E. F. Pinel, J. de la Venta, A. Quesada, V. Bouzas, J. F. Fernández, J. J. Romero, M. S. M. González, and J. L. Costa-Kräme, J. Appl. Phys. 105, 013925 (2009).

[187] J. F. Ziegler, J. P. Biersack, and U. Littmark, The Stopping and Range of Ions in Solids (Pergamon, New York, 1985).

[188] N. Hayashi, I. Sakamoto, T. Toriyama, H. Wakabayashi, T. Okada, and K. Kuriyama, Surf. Coat. Technol. 169-170, 540 (2003).

[189] Z. Mao, Z. He, D. Chen, W. Y. Cheung, and S. P. Wong, Solid State Commun. 142, 329 (2007).

[190] J. F. Hu, Z. I. Zhang, M. Zhao, H. W. Qin, and M. H. Jiang, Appl. Phys. Lett. 93, 192503 (2008).

[191] J. I. Beltran, C. Monty, L. Balcells, and C. Martinez-Boubeta, Solid State Commun. 149, 1654 (2009).

[192] C. Martinez-Boubeta, J. I. Beltran, L. Balcells, Z. Konstantinovic, S. Valencia, D. Schimitz, J. Cornil, and B. Martinez, (unpublished) (2010).

[193] E. I. Rashba, Phys. Rev. B 62, R16267 (2000).

[194] S. F. Alvarado, Phys. Rev. Lett. 75, 513 (1995). 
[195] D. J. Monsma, R. Vlutters, and J. C. Lodder, Science 281, 407 (1998).

[196] H. E. Weaver, J. Phys. Chem. Solids 11, 274 (1959).

[197] K. Fukushima and S. Shibagaki, Thin Solid Films 315, 238 (1998).

[198] S. Dai, H. Lu, F. Chen, Z. Chen, Z. Y. Ren, and D. H. L. Ng, Appl. Phys. Lett. 80, 3545 (2002).

[199] A. Leitner, C. T. Rogers, J. C. Price, D. A. Rudman, and D. R. Herman, Appl. Phys. Lett. 72, 3065 (1998).

[200] URL www.mtixtl.com/crystalssubstratesa-z.aspx.

[201] M. Ziese, U. Köhler, A. Bollero, R. Höhne, and P. Esquinazi, Phys. Rev. B 71, 180406(R) (2005).

[202] D. C. Kundaliya, S. B. Ogale, L. F. Fu, S. J. Welz, J. S. Higgins, G. Langham, S. Dhar, N. D. Browning, and T. Venkatesan, J. Appl. Phys. 99, 08K304 (2006).

[203] P. Han, H. bin Lu, K. juan Jin, J.-F. Jia, J. Qiu, C. lian Hu, and L. Liao, Physica B 404, 1332 (2009).

[204] H. Kato, T. Okuda, Y. Okimoto, Y. Tomioka, Y. Takenoya, A. Ohkubo, M. Kawasaki, and Y. Tokura, Appl. Phys. Lett. 81, 328 (2002).

[205] J. Longo and R. Ward, J. Am. Chem. Soc. 83, 2816 (1961).

[206] K. I. Kobayashi, T. Kimura, H. Sawada, K. Terakura, and Y. Tokura, Nature 395, 677 (1998).

[207] K. I. Kobayashi, T. Kimura, Y. Tomioka, H. Sawada, K. Terakura, and Y. Tokura, Phys. Rev. B 59, 11159 (1999).

[208] A. Maignan, B. Raveau, C. Martin, and M. Hervieu, J. Solid State Chem. 144, 224 (1999).

[209] J. A. Alonso, M. T. Casais, M. J. Martinez-Lope, J. L. Martinez, P. Velasco, A. Muñoz, and M. T. Fernanderz-Diaz, Chem. Mater. 12, 161 (2000).

[210] C. Ritter, M. R. Ibarra, L. Morellon, J. Blasco, J. García, and J. M. D. Teresa, J. Phys.: Condens. Matter 12, 8295 (2000).

[211] D. Serrate, J. M. DeTeresa, and M. R. Ibarra, J. Phys.: Condens. Matter 19, 023201 (2007). 
[212] F. K. Patterson, C. W. Moeller, and R. Word., Inor. Chem. 2, 196 (1963).

[213] A. Arulraj, K. Ramesha, J. Gopalakrishnan, and C. N. R. Rao, J. Solid State Chem. 155, 233 (2000).

[214] J. B. Philipp, D. Reisinger, M. Schonecke, A. Marx, A. Erb, L. Alff, R. Gross, and J. Klein, Appl. Phys. Lett. 79, 3654 (2001).

[215] A. S. Ogale, S. B. Ogale, R. Ramesh, and T. Venkatesan, Appl. Phys. Lett. 75, 537 (1999).

[216] I. V. Solovyev, Phys. Rev. B 65, 144446 (2002).

[217] L. Balcells, J. Navarro, M. Bibes, A. Roig, B. Martínez, and J. Fontcuberta, Appl. Phys. Lett. 78, 781 (2001).

[218] J. M. DeTeresa, J. M. Michalik, J. Blasco, P. A. Algarabel, M. R. Ibarra, C. Kapusta, and U. Zeitler, Appl. Phys. Lett. 90, 252514 (2007).

[219] T. Manako, M. Izumi, Y. Konishi, K. Kobayashi, M. Kawasaki, and Y. Tokura, Appl. Phys. Lett. 74, 2215 (1999).

[220] W. Westerburg, D. Reisinger, and G. Jakob, Phys. Rev. B 62, R767 (2000).

[221] M. Besse, F. Pailloux, A. Barthelemy, K. Bouzehouane, A. Fert, J.Olivier, O. Durand, F. Wyczisk, R. Bisaro, and J. P. Contour, J. Crystal Growth 241, 448 (2002).

[222] D. Sanchez, M. Garcia-Hernandez, N. Auth, and G. Jakob, J. Appl. Phys. 96, 2736 (2004).

[223] T. Fix, G. Versini, J. L. Loison, S. Colis, G. Schmerber, G. Pourroy, and A. Dinia, J. Appl. Phys. 97, 024907 (2005).

[224] J. B. Philipp, D. Reisinger, M. Schonecke, M. Opel, A. Marx, A. Erb, L. Alff, and R. Gross, J. Appl. Phys. 93, 6853 (2003).

[225] H. Asano, N. Kozuka, A. Tsuzuki, and M. Matsui, Appl. Phys. Lett. 85, 263 (2004).

[226] S. Geprägs, F. D. Czeschka, M. Opel, S. T. B. Goennenwein, W. Yu, W. Mader, and R. Gross, J. Magn. Magn. Mater. 321, 2001 (2009).

[227] M. R. Castell, Surf. Sci. 505, 1 (2002).

[228] A. N. Khodan, S. Guyard, J. P. Contour, D. B. Crété, E. Jacquet, and K. Bouzehouane, Thin Solid Films 515, 6422 (2007). 
[229] V. M. Bermudez and V. H. Ritz, Chem. Phys. Lett 73, 160 (1980).

[230] R. G. Egdell and P. D. Naylor, Chem. Phys. Lett 91, 200 (1982).

[231] T. Kubo and H. Nozoye, Surf. Sci. 542, 177 (2003).

[232] J. Orna, L. Morellón, P. A. Algarabel, J. A. Pardo, C. Magen, M. Varela, S. J. Pennycook, J. M. D. Teresa, and M. R. Ibarra, J. Magn. Magn. Mater. 322, 1217 (2009).

[233] J. M. Michalik, J. M. D. Teresa, C. Ritter, J. Blasco, D. Serrate, M. R. Ibarra, C. Kapusta, J. Freudenberger, and N. Kozlova, Europhys. Lett 78, 17006 (2007).

[234] F. Albertini, L. Nasi, F. Casoli, S. Fabbrici, P. Luches, A. Rota, and S. Valeri, J. Magn. Magn. Mater. 316, e158 (2007).

[235] F. Casoli, Ph.D. thesis, Università degli study di Parma, Facoltà di Scienze Matematiche Fisiche Naturali (2005), cum laudae.

[236] F. Casoli, L. Nasi, F. Albertini, S. Fabbrici, C. Bocchi, F. Germini, P. Luches, A. Rota, and S. Valeri, J. Appl. Phys. 103, 043912 (2008).

[237] F. Casoli, F. Albertini, L. Nasi, S. Fabbrici, R. Cabassi, F. Bolzoni, and C. Bocchi, Appl. Phys. Lett. 92, 142506 (2008).

[238] D. Weller, A. Moser, L. Folks, M. E. Best, W. Lee, M. F. Toney, M. Schwickert, J.-U. Thiele, and M. F. Doerner, IEEE Trans. Magn. 36, 10 (2000).

[239] H. N. Bertram and M.Williams, IEEE Trans. Magn. 36, 4 (2000).

[240] D. E. Laughlin, S. Kumar, Y. Peng, and A. G. Roy, IEEE Trans. Magn. 41, 719 (2005).

[241] O. A. Ivanov, L. V. Solina, V. A. Demshina, and L. M. Magat, Fiz. metal. metalloved. 35, 92 (1973).

[242] P. Fredriksson and B. Sundman, CALPHAD 25, 535 (2001).

[243] D. P. Nguyen, S.-Y. O, C. W. Park, K. Shin, C. G. Lee, T. Shimozaki, and T. Okino., J. Magn. Magn. Mater. 320, e264 (2008).

[244] T. Shima, K. Takanashi, Y. K. Takahashi, and K. Hono, Appl. Phys. Lett. 81, 1050 (2002).

[245] Y. K. Takahashi, M. Ohnuma, and K. Hono, Jpn. J. Appl. Phys. 40, L1367 (2001). 
[246] C. P. Luo and D. J. Sellmyer, Appl. Phys. Lett. 75, 3162 (1999).

[247] S. R. Lee, S. Yang, Y. K. Kim, and J. G. Na, Appl. Phys. Lett. 78, 4001 (2001).

[248] A. Cebollada, D. Weller, J. Sticht, G. R. Harp, R. F. C. Farrow, R. F. Marks, R. Savoy, and J. C. Scott, Phys. Rev. B 50, 3419 (1994).

[249] T. Seki, T. Shima, K. Takanashi, Y. Takahashi, E. Matsubara, Y. K. Takahashi, and K. Hono, J. Appl. Phys. 96, 1127 (2004).

[250] C. Feng, Q. Zhan, B. Li, J. Teng, M. Li, Y. Jiang, and G. Yu, Appl. Phys. Lett. 93, 152513 (2008).

[251] Y. S. Yu, H. B. li, W. L. Li, M. Liu, Y. M. Zhang, and W. D. Fei, J. Phys. D: Appl. Phys. 41, 245003 (2008).

[252] T. Maeda, T. Kai, A. Kikitsu, T. Nagase, and J. I. Akiyama, Appl. Phys. Lett. 80, 2147 (2002).

[253] Y. F. Ding, J. S. Chen, E. Liu, C. J. Sun, and G. M. Chow, J. Appl. Phys. 97, 10H303 (2005).

[254] T. Suzuki, K. Harada, N. Honda, and K. Ouchi, J. Magn. Magn. Mater. 193, 85 (1999).

[255] C. H. Lai, C. H. Yang, and C. C. Chiang, Appl. Phys. Lett. 83, 4550 (2003).

[256] Y. K. Takahashi, K. Hono, T. Shima, and K. Takanashi, J. Magn. Magn. Mater. 267, 248 (2003).

[257] K. Gosh, S. B. Ogale, S. P. Pai, M. Robson, E. Li, I. Jin, Z. Dong, R. L. Greene, R. Ramesh, T. Venkatesan, et al., Appl. Phys. Lett. 73, 689 (1998).

[258] X. W. Li, A. Gupta, G. Xiao, W. Qian, and V. P. Dravid, Appl. Phys. Lett. 73, 3282 (1998).

[259] M. Bibes and A. Barthélémy, IEEE Trans. Electron Devices 54, 1003 (2007).

[260] T. Kiyomura, Y. Maruo, and M. Gomi, J. Appl. Phys. 88, 4768 (2000).

[261] W. Kim, K. Kawaguchi, N. Koshizaki, M. Sohma, and T. Matsumoto, J. Appl. Phys. 93, 8032 (2003). 
[262] X. Jin, C. McEvoy, and I. V. Shvets, J. Magn. Magn. Mater. 286, 128 (2005).

[263] P. J. van der Zaag, P. J. H. Bloemen, J. M. Gaines, R. M. Wolf, P. A. A. van der Heijden, R. J. M. van der Veerdonk, and W. J. M. de Jonge, J. Magn. Magn. Mater. 211, 301 (2000).

[264] T. Kado, H. Saito, and K. Ando, J. Appl. Phys. 101, 09J511 (2007).

[265] F. Greullet, E. Snoeck, C. Tiusan, M. Hehn, D. Lacour, O. Lenoble, C. Magen, and L. Calmels, Appl. Phys. Lett. 92, 053508 (2008).

[266] K. S. Yoon, J. H. Koo, Y. H. Do, K. W. Kim, C. O. Kim, and J. P. Hong, J. Magn. Magn. Mater. 285, 125 (2005).

[267] D. Reisinger, P.Majewski, M. Opel, L. Alff, and R. Gross, condmat/0407725 (2004).

[268] S. Parkin, X. Jiang, C. Kaiser, A. Panchula, K. Roche, and M. Samant, Proc. IEEE 91, 661 (2003).

[269] H. Matsuda, M. Takeuchi, H. Adachi, M. Hiramoto, N. Matsukawa, A. Odagawa, K. Setsune, and H. Sakakima, Jpn. J. Appl. Phys. 41, L387 (2002).

[270] R. Moons, S. Blässer, J. Dekoster, A. Vantomme, J. D. Wachter, and G. Langouche, Thin Solid Films 324, 129 (1998).

[271] P. Granberg, P. Isberg, T. Baier, B. Hjörvarsson, and P. Nordblad, J. Magn. Magn. Mater. 195, 1 (1999).

[272] P. A. A. van der Heijden, P. J. H. Bloemen, J. M. Metselaar, R. M. Wolf, J. M. Gaines, J. T. W. M. van Eemeren, P. J. van der Zaag, and W. J. M. de Jonge, Phys. Rev. B 55, 11569 (1997).

[273] J. Orna, L. Morellón, P. A. Algarabel, J. A. Pardo, S. Sangiao, C. Magen, E. Snoeck, J. M. D. Teresa, and M. R. Ibarra, IEEE Trans. Magn 44, 2862 (2008).

[274] S. Cardoso, C. Cavaco, R. Ferreira, L. Pereira, M. Rickart, P. P. Freitas, N. Franco, J. Gouveia, and N. P. Barradas, J. Appl. Phys. 97, 10C916 (2005).

[275] Z. G. Zhang, P. P. Freitas, A. R. Ramos, N. P. Barradas, and J. C. Soares, J. Appl. Phys. 91, 8786 (2002). 
ISBN 978-84-15031-68-0

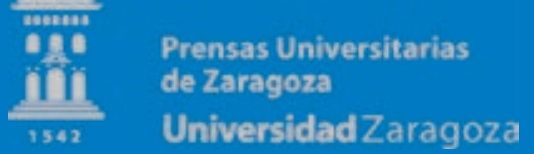

